Prepared in cooperation with the National Park Service

\title{
Development of Monitoring Protocols to Detect Change in Rocky Intertidal Communities of Glacier Bay National Park and Preserve
}

Open-File Report 2010-1283 



\section{Development of Monitoring Protocols to Detect Change in Rocky Intertidal Communities of Glacier Bay National Park and Preserve}

By Gail V. Irvine

Prepared in cooperation with the National Park Service

Open-File Report 2010-1283

U.S. Department of the Interior

U.S. Geological Survey 


\section{U.S. Department of the Interior \\ KEN SALAZAR, Secretary}

\section{U.S. Geological Survey \\ Marcia K. McNutt, Director}

U.S. Geological Survey, Reston, Virginia: 2010

For more information on the USGS—-the Federal source for science about the Earth, its natural and living resources, natural hazards, and the environment, visit http://www.usgs.gov or call 1-888-ASK-USGS.

For an overview of USGS information products, including maps, imagery, and publications, visit http://www.usgs.gov/pubprod

To order this and other USGS information products, visit http://store.usgs.gov

Suggested citation:

Irvine, G.V., 2010, Development of monitoring protocols to detect change in rocky intertidal communities of Glacier Bay National Park and Preserve: U.S. Geological Survey Open-File Report 2010-1283, 140p.

Any use of trade, product, or firm names is for descriptive purposes only and does not imply endorsement by the U.S. Government.

Although this report is in the public domain, permission must be secured from the individual copyright owners to reproduce any copyrighted material contained within this report. 


\section{Contents}

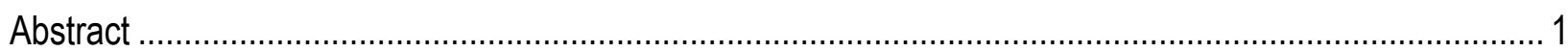

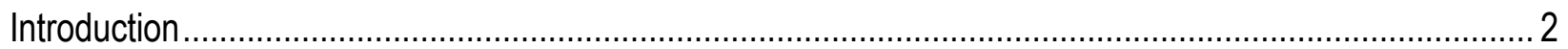

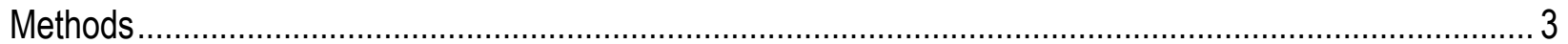

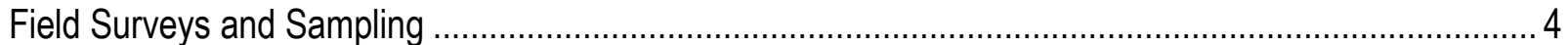

Aerial Surveys and Description of Habitat Types ...................................................................... 4

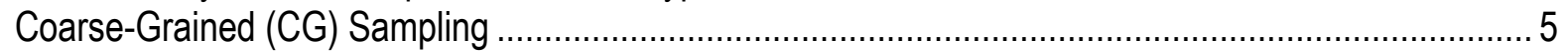

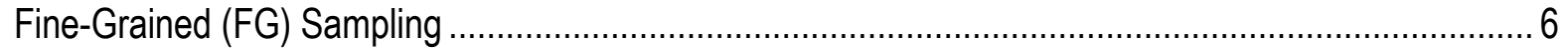

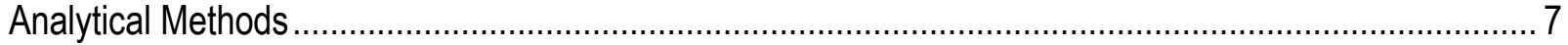

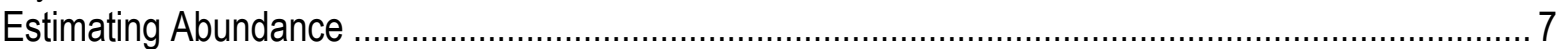

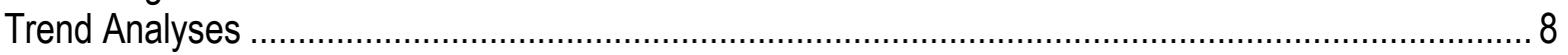

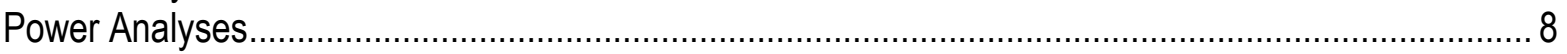

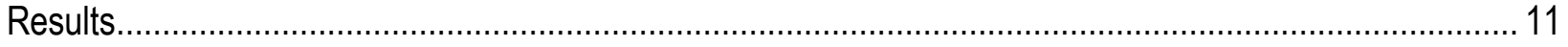

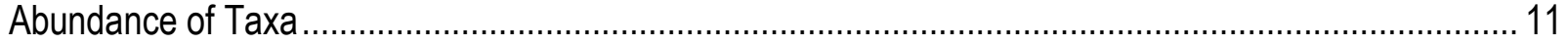

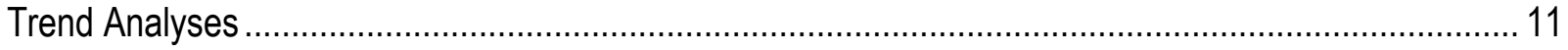

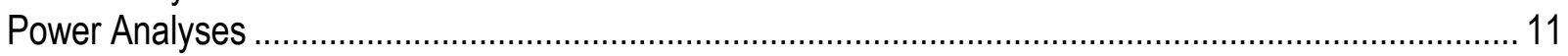

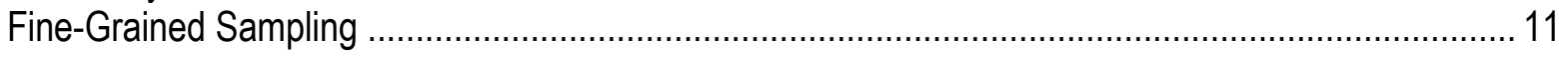

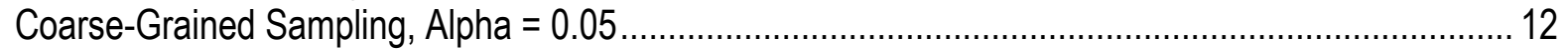

Coarse-Grained Sampling, Varying Alpha............................................................................... 13

Comparison of Coarse-Grained and Fine-Grained Sampling ........................................................ 13

TerraStat Power Reanalyses............................................................................................... 14

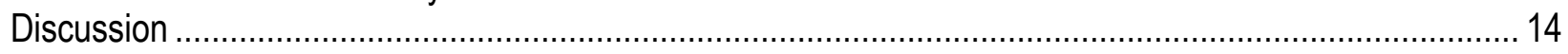

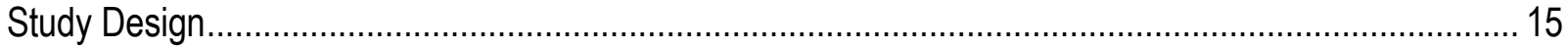

Comparison with Other Intertidal Monitoring Designs ..................................................................... 17

Power, Trend Detection, and Impact Analysis .......................................................................... 18

Variation in the Ability to Detect Change among Taxa................................................................. 19

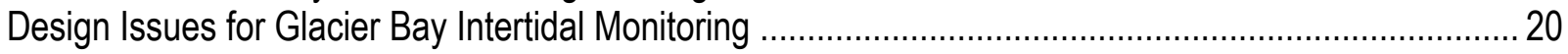

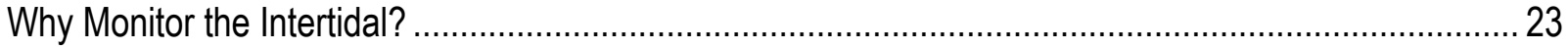

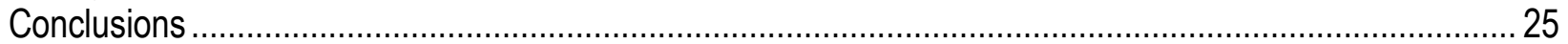

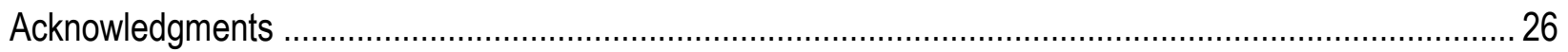

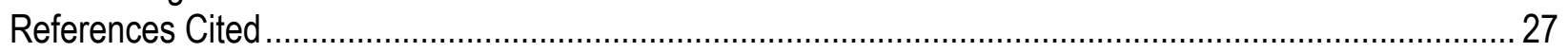

Appendix A. Methods for the 1997 Sampling, including Aerial Survey Standard Operating Procedures ..... 61

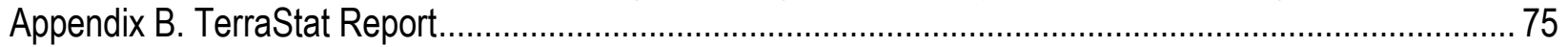

Appendix C. Additional Power Analyses ....................................................................................... 91

Appendix D. Standard Operating Procedures for Coarse-Grained Sampling ……................................. 119

Appendix E. Glacier Bay Intertidal Monitoring Access Database ............................................................ 131 


\section{Figures}

Figure 1. Location of Glacier Bay National Park and Preserve in southeast Alaska ................................ 30

Figure 2. Locations and segment numbers of the coarse-grained and fine-grained sites in Glacier Bay National Park and Preserve........................................................................................................ 31

Figure 3. Layout of the various sampling methods used during the fine-grained surveys......................... 32

Figure 4. Quadrat used for point-contact sampling at the fine-grained sampling level.............................. 33

Figure 5. Comparison of the power of three point-intercept sampling methods conducted as part of the fine-grained sampling protocol in 1997

Figure 6. Power to detect a 10 percent decrease in the predominant sessile species as related to the number of transects sampled

Figure 7. Power to detect population trends based on 4 years of data (1997-2001) in A. barnacles; B.

Mytilus; and C. Fucus

Figure 8. Power to detect population trends based on 3 years of data (1998-2001) in A. barnacles; B.

Mytilus; and C. Fucus

Figure 9. Power to detect decreases in Mytilus populations as related to the number of points

sampled per meter of each transect

Figure 10. Power to detect decreases in Fucus populations as related to the number of points

sampled per meter of each transect

Figure 11. Power to detect decreases in barnacle populations as related to the number of points sampled per meter of each transect.

Figure 12. Power to detect a decrease in Mytilus populations as a function of the number of transects sampled at each site

Figure 13. Power to detect a decrease in Mytilus populations in relation to the number of sites

Figure 14. Change in the power to detect a decrease in Mytilus populations as a result of varying the number of points sampled per meter with respect to 25,20 , and 15 sites.

Figure 15. Power to detect trends of differing magnitudes at three levels of alpha $(\alpha=0.05,0.10,0.20) . .44$

Figure 16. Comparison in the power of fine-grained and coarse-grained vertical transect

sampling to detect a 10 percent decrease in Mytilus, Fucus, and barnacles.

Figure 17. Comparison of the power of coarse-grained and fine-grained sampling methods

to detect trends in the predominant sessile species 46

Figure 18. Statistical power comparison for Mytilus with 25 sites ........................................................4 47

Figure 19. Statistical power comparison for Mytilus with 15 sites. ......................................................... 48

Figure 20. Statistical power comparison for Fucus ...................................................................... 49

Figure 21. Statistical power comparison for barnacles................................................................... 50

Figure 22. Mean percent cover of the predominant sessile species at Berg Bay......................................51 


\section{Tables}

Table 1. Parameters used in the power analyses.........................................................................52

Table 2. The number of hits (counts) of a species or taxon during each year of sampling along the vertical transects.

Table 3. Trend results by site for percent cover of Mytilus. ............................................................... 56

Table 4. Trend results by site for percent cover of Fucus..............................................................5

Table 5. Trend results by site for percent cover of barnacles..........................................................5

Table 6. Conversion of short-term trends, expressed as percent change per year, into long-term trends.59

\section{Conversion Factors}

\begin{tabular}{|c|c|c|}
\hline Multiply & By & To obtain \\
\hline \multicolumn{3}{|c|}{ Length } \\
\hline foot $(\mathrm{ft})$ & 0.3048 & meter $(\mathrm{m})$ \\
\hline \multicolumn{3}{|c|}{ Area } \\
\hline acre & 4,047 & square meter $\left(\mathrm{m}^{2}\right)$ \\
\hline acre & 0.4047 & hectare (ha) \\
\hline acre & 0.4047 & square hectometer $\left(\mathrm{hm}^{2}\right)$ \\
\hline acre & 0.004047 & square kilometer $\left(\mathrm{km}^{2}\right)$ \\
\hline square foot $\left(\mathrm{ft}^{2}\right)$ & 929.0 & square centimeter $\left(\mathrm{cm}^{2}\right)$ \\
\hline square foot $\left(\mathrm{ft}^{2}\right)$ & 0.09290 & square meter $\left(\mathrm{m}^{2}\right)$ \\
\hline \multicolumn{3}{|l|}{ SI to Inch/Pound } \\
\hline Multiply & By & To obtain \\
\hline \multicolumn{3}{|c|}{ Length } \\
\hline centimeter $(\mathrm{cm})$ & 0.3937 & inch (in.) \\
\hline millimeter $(\mathrm{mm})$ & 0.03937 & inch (in.) \\
\hline meter $(\mathrm{m})$ & 3.281 & foot $(\mathrm{ft})$ \\
\hline kilometer (km) & 0.6214 & mile (mi) \\
\hline kilometer $(\mathrm{km})$ & 0.5400 & mile, nautical (nmi) \\
\hline meter $(\mathrm{m})$ & 1.094 & yard $(y d)$ \\
\hline \multicolumn{3}{|c|}{ Area } \\
\hline hectare (ha) & 2.471 & acre \\
\hline square meter $\left(\mathrm{m}^{2}\right)$ & 0.0002471 & acre \\
\hline square meter $\left(\mathrm{m}^{2}\right)$ & 10.76 & square foot $\left(\mathrm{ft}^{2}\right)$ \\
\hline
\end{tabular}




\section{List of Acronyms}

$\begin{array}{ll}\text { 3D } & \text { Three dimensional } \\ \text { BACI } & \text { Before-After-Control-Impact (design) } \\ \text { BACIP } & \text { Before-After-Control-Impact-Paired (design) } \\ \text { CG } & \text { Coarse-grained sampling } \\ \text { FG } & \text { Fine-grained sampling } \\ \text { GRTS } & \text { Generalized random-tesselation stratified (survey design) } \\ \text { MARINe } & \text { Multi-Agency Rocky Intertidal Network } \\ \text { MHHW } & \text { Mean higher high water } \\ \text { MLLW } & \text { Mean lower low water } \\ \text { NHP } & \text { National Historical Park } \\ \text { NP } & \text { National Park } \\ \text { NP\&P } & \text { National Park and Preserve } \\ \text { NPS } & \text { National Park Service } \\ \text { PISCO } & \text { Partnership for Interdisciplinary Study of Coastal Oceans } \\ \text { USGS } & \text { U.S. Geological Survey } \\ \text { WEST, Inc. } & \text { Western EcoSystems Technology, Inc. }\end{array}$

\section{List of Symbols}

a-alpha Type I error level (probability of rejecting the null hypothesis when it is true), acceptable error level

$\beta$ - beta $\quad 1-\beta=$ power of a statistical test (the probability of rejecting the null hypothesis when it is false and should be rejected)

- degrees

$<\quad$ less than

$\leq \quad$ less than or equal to

$>\quad$ greater than

$\geq \quad$ greater than or equal to 


\title{
Development of Monitoring Protocols to Detect Change in Rocky Intertidal Communities of Glacier Bay National Park and Preserve
}

By Gail V. Irvine

\begin{abstract}
Glacier Bay National Park and Preserve in southeastern Alaska includes extensive coastlines representing a major proportion of all coastlines held by the National Park Service. The marine plants and invertebrates that occupy intertidal shores form highly productive communities that are ecologically important to a number of vertebrate and invertebrate consumers and that are vulnerable to human disturbances. To better understand these communities and their sensitivity, it is important to obtain information on species abundances over space and time. During field studies from 1997 to 2001, I investigated probability-based rocky intertidal monitoring designs that allow inference of results to similar habitat within the bay and that reduce bias. Aerial surveys of a subset of intertidal habitat indicated that the original target habitat of bedrock-dominated sites with slope less than or equal to 30 degrees was rare. This finding illustrated the value of probability-based surveys and led to a shift in the target habitat type to more mixed rocky habitat with steeper slopes. Subsequently, I investigated different sampling methods and strategies for their relative power to detect changes in the abundances of the predominant sessile intertidal taxa: barnacles -Balanomorpha, the mussel Mytilus trossulus and the rockweed Fucus distichus subsp. evanescens. I found that lowerintensity sampling of 25 randomly selected sites (= coarse-grained sampling) provided a greater ability to detect changes in the abundances of these taxa than did more intensive sampling of 6 sites (= fine-grained sampling). Because of its greater power, the coarse-grained sampling scheme was adopted in subsequent years. This report provides detailed analyses of the 4 years of data and evaluates the relative effect of different sampling attributes and management-set parameters on the ability of the sampling to detect changes in the abundances of these taxa. The intent was to provide managers with information to guide design choices for intertidal monitoring. I found that the coarse-grained surveys, as conducted from 1998 to 2001, had power ranging from 0.68 to 1.0 to detect 10 percent annual changes in the abundances of these predominant sessile species. The information gained through intertidal monitoring would be useful in assessing changes due to climate (including ocean acidification), invasive species, trampling effects, and oil spills.
\end{abstract}




\section{Introduction}

For Glacier Bay and many other Alaska national parks, the National Park Service's mission "to protect unimpaired" the lands and resources within its jurisdiction is complicated by two main factors. First is the lack of knowledge of the status of park resources, their dynamics through time, and the relationships among species and processes. This lack of knowledge makes it difficult to separate natural variation from anthropogenic effects, thus hampering the ability of managers to know when and if they can intervene to effect change. The second complicating factor is large spatial scale. The marine coastline of Glacier Bay National Park and Preserve (NP\&P; fig. 1) spans about 1,720 km (Irvine and others, 1994) and for Glacier Bay proper-the focus of this study-1,109 km (Irvine, 1998). The Glacier Bay coastline comprises about 40 percent of the estimated 4,210 km coastline held by all national parks in Alaska. Because the Alaskan national parks contain almost 70 percent of the marine coastline of all US national parks (Irvine and others, 1994), Glacier Bay has a disproportionately large share of the marine coastlines managed by the NPS in both Alaska and the USA.

The 1989 Exxon Valdez oil spill in south-central Alaska contaminated more than 2,000 $\mathrm{km}$ of coastal habitat and associated biota, including areas of Kenai Fjords and Katmai NP\&Ps. This spill illustrated the vulnerability of these coastal environments to anthropogenic effects. Following that event, the Alaska region of the NPS created a Coastal Programs Division for the purpose of developing inventory and monitoring programs for the extensive national park coastlines in Alaska. In 1996, I received funding to develop monitoring protocols and studies that address landscape-scale variation in the coastal biological communities of national parks. Based on jurisdictional, budget and logistical considerations, I was asked to concentrate effort at Glacier Bay NP\&P.

Monitoring involves determination of the abundance or status of species through time, accomplished by repeated measurements or counts. The ability to detect change or trends depends on the design of the monitoring, sample size, level of certainty selected, and the natural variability in spatial and temporal patterns of species abundances. As the variability in abundance or any other parameter increases, the ability to detect change in that parameter decreases, given equal sampling effort. Therefore, it is more difficult to detect trends in species that are rare or whose abundance is highly variable. Sample variation may be reduced and the power to detect change increased by stratifying the populations and communities along known gradients of variation, or by increasing sampling effort. Increased sampling costs more, while stratified sampling reduces the ability to make inferences beyond the strata sampled, unless all populations are accounted for in the stratification process.

One of the challenges faced by resource managers in designing and implementing a monitoring program is how to balance the need to sample across broad physical scales for greater spatial inference versus the need to sample intensively enough to detect temporal changes in biological communities. Intensive sampling is often conducted over smaller scales, and inference may be limited to selected habitat types, sites, plots, and species. 
The major objective of this study was to develop a probability-based approach to monitoring intertidal assemblages inhabiting protected rocky substrates, so that the results of the sampling could be extended to similar habitat within Glacier Bay proper. Initially, aerial surveys were conducted to classify coastal segments and determine the locations of these rocky substrates. Based on findings from the aerial surveys, the target habitat type was defined as: coastal areas comprised of bedrock ( $>1$ percent) and/or $\geq 76$ percent cobble/boulder substrate, with slopes $\leq 60^{\circ}$. Extensive sampling was conducted at 25 randomly selected sites under the coarse-grained sampling regime. More intensive sampling of 6 sites from the lower half of the bay (a subset of the 25 sites), constituted the fine-grained sampling. These fine-grained sites were all cobble/boulder-dominated sites.

Sampling primarily targeted all macro sessile species and large mobile invertebrates. Analyses to test the power of the sampling to detect changes in abundance of species concentrated on the three most abundant sessile taxa in the sampled habitat type: barnacles, the mussel Mytilus trossulus, and the rockweed Fucus distichus subsp. evanescens. Continued sampling of all sessile species will allow detection of changes in the distribution and abundance of sessile species spatially and temporally within Glacier Bay proper.

The results of the sampling conducted from 1997 to 2001 have inference to the selected habitat type within Glacier Bay proper for the coarse-grained sampling, and to a narrower band of cobble/boulder habitat within Glacier Bay for the fine-grained sampling.

In this final report, I summarize the study, review key findings presented in earlier reports, and describe final results leading to a recommended protocol for detecting change in the abundance of key sessile species of rocky intertidal communities in Glacier Bay.

\section{Methods}

Planning and fieldwork for this study were conducted from 1996 to 2001. In 1996, I held meetings with Glacier Bay staff to discuss goals for the project, potential stressors affecting the coastal areas, and approaches to the study. We decided to focus the study in Glacier Bay proper because the park staff rated protected rocky intertidal habitat as their top choice for monitoring, and they were most concerned about potential effects from oil spills and the immigration of sea otters (Enhydra lutris), both of which they thought were more likely to affect communities within the bay. Fieldwork was carried out in 1997, 1998, 1999, and 2001.

This study used a probability-based, multilevel design that included:

a. Aerial surveys of a systematically selected subset of coastal segments to characterize their habitat attributes, assess the frequency of different habitat types, and provide a pool of sites of known habitat type for further sampling,

b. Coarse-grained (CG) or lower intensity sampling of a large number of randomly selected sites of a selected habitat type, and

c. Fine-grained (FG) or more intensive sampling of a few sites of cobble/boulder habitat, selected as a subset of the CG sampling sites; the FG sampling also tested the efficacy of additional sampling methods. 
I examined the ability of the CG and FG sampling plans to detect trends in the predominant sessile species through power analyses. Power can be defined as the ability to detect a change when one is occurring. Statistically, power is the probability of rejecting the null hypothesis when it is false and should be rejected (Zar, 1984). As part of the power analyses, sampling attributes, such as the number of sites, transects, and intensity of point sampling, were varied to examine their effect on the power of particular sampling plans to detect change. Additionally, I examined the interplay between sampling attributes and management-set parameters (that is; the level of annual change to be detected and error levels) on power (table 1).

The methods used in the three levels of surveys and sampling listed above are described in detail in Irvine (1998) and in appendix A. They are reviewed below, followed by description of the analytical methods.

\section{Field Surveys and Sampling}

\section{Aerial Surveys and Description of Habitat Types}

The goal of the aerial surveys was to characterize intertidal habitat types and their frequencies in Glacier Bay. The categorical abundances of substrate and spatially dominant sessile biota, as well as slope, were described for each segment surveyed.

The first step in defining a set of sites to survey was the division of a digitized coastline of Glacier Bay NP\&P (Geiselman and others, 1997) into 200-m length segments. The 1,109 km coast of Glacier Bay proper yielded 5,545 segments. I estimated that 250 segments could be aerially surveyed during one tide series. Beginning with a random start in Glacier Bay proper, each 23rd segment was selected to be surveyed. Then, from a fixed-wing plane, 241 of the 250 segments were classified categorically for habitat type, slope, and biota (Appendix A: Aerial Survey Standard Operating Procedure). Nine of the segments were found to be inappropriate and were not surveyed; details on the exclusions are in appendix A. Summary data from the aerial surveys were reported in Irvine (1998). These described segments then formed a pool of sites with known habitat types that were available for subsequent stages of the intertidal sampling.

Prior to the aerial surveys, the target habitat type had been defined as predominantly ( $\geq$ 76 percent cover) bedrock substrate with slopes $\leq 30^{\circ}$. The results of the aerial surveys led to a change in the targeted habitat type for monitoring, because only one of the 241 segments surveyed had these characteristics. Even increasing the acceptable slope to $\leq 60^{\circ}$ added only two more segments to the pool. Thus, in consultation with NPS and Center staff, I decided to define the selected habitat type as segments dominated by cobbles and boulders ( $\geq 76$ percent cover) and/or having a bedrock component ( $\geq 1$ percent); slope was defined as $\leq 60^{\circ}$. This created a pool of 111 segments, which formed the sampling frame of the target habitat type. 


\section{Coarse-Grained (CG) Sampling}

From the pool of 111 segments with the target habitat type, 30 sites were randomly selected for CG sampling (fig. 2). Five sites were eliminated after the initial draw because they were either too steep, were not true beaches (for example, no land exposed at high tide), or were not accessible because the site was in a wildlife protection area (appendix A).

CG sampling was conducted in 1997, 1998, 1999, and 2001. Each 200-m-long site was located in the field using global positioning system coordinates determined from the original segmentation of the digitized coastline. The area sampled was the area between mean higher high water (MHHW), defined by biological characteristics, and the 0 -m tide level (mean lower low water, MLLW), set with information from the tide program Tides \& Currents for Windows, version 2.5a (Nautical Software, Inc., 1997). A horizontal segment line delimiting the upper bound of the site was laid along the MHHW contour. Six vertical-line transects running parallel to the elevational gradient and perpendicular to the shoreline were laid from MHHW to the $0-\mathrm{m}$ tide level. The start of the first vertical transect was determined randomly within the first $33 \mathrm{~m}$ of the horizontal segment line; the succeeding transect lines were laid out systematically, at 33-m intervals with respect to the first. If a vertical transect fell on a section of the segment that was unsampleable due to steep slope or freshwater input, then that transect and the remaining transects were shifted the horizontal distance of the unsampleable area to the right, facing shore. The locations of the transects were randomized again each year.

Two types of sampling, each targeting different types of species, were conducted along the vertical transects. Sessile species were targeted by 3-dimensional (3D), point-intercept sampling along the vertical transects. All species (sessile and mobile), including multiple layers of the same species under the point were recorded, from top to bottom, until the first substrate was found. The substrate also was recorded. Large mobile invertebrate species (starfish, sea urchins, and large chitons) were targeted in band surveys that extended $1 \mathrm{~m}$ to each side of the vertical transect line. Species identifications were made to the lowest taxonomic level possible in the field during all surveys (appendix A).

In 1997, the CG sampling used a base sampling intensity of 1 point $/ \mathrm{m}$ along each vertical transect. Although my original plan was to sample at 5 points $/ \mathrm{m}$, I reset the sampling intensity to 1 point $/ \mathrm{m}$ based on the time it took to do the 3D sampling in an initial field test and the objective of sampling one site per day. As field crews became more experienced and most sites were found to have shorter transects than those in the field test, I increased the sampling intensity to 2 points/m (x.0-m and x.6-m marks). The x.6-m mark was selected as I anticipated that pointintercept sampling at the FG sites would be conducted at 5 points $/ \mathrm{m}$, each $20 \mathrm{~cm}$, and that future sampling of CG sites likely would be conducted at $20-\mathrm{cm}$ intervals. At a few sites, sampling was conducted at $>2$ points $/ \mathrm{m}$. Thus, in 1997,17 of the 25 sites were sampled at $\geq 2$ points $/ \mathrm{m}$ and 8 sites (sites 62, 63, 69, 216, 217, 218, 223, and 224) were sampled at 1 point $/ \mathrm{m}$. Beginning in 1998 , I increased the sampling intensity to 5 points/m at all 25 sites to increase the probability of detecting less-common species and to increase the precision of the estimates. 


\section{Fine-Grained (FG) Sampling}

Fine-grained sampling was conducted in 1997 at 6 of the 25 CG sites (fig. 2; appendix A). I narrowed the focus of the FG site selection in two ways. First, I narrowed the geographical focus to allow easier access to the sites by skiff from park headquarters at Bartlett Cove (located slightly to the east of site 59; fig. 2), because the more intensive FG sampling necessitated multiple days of effort at each site. Second, I decided, in consultation with Center and Park staff, to concentrate efforts on predominantly cobble/boulder sites; only those sites with $\geq 76$ percent cobble/boulder substrate were considered for inclusion.

Six sites were selected from the CG suite of sites that fit these habitat and geographic parameters. Below the juncture of the two arms in Glacier Bay there were eight CG sites that had been defined as predominately cobble/boulder habitat during the aerial surveys. One of those, site 88, the most distant from Bartlett Cove in this band, was discarded because the CG surveys had revealed that little cobble/boulder was present. The most southerly geographic site also was discarded. This resulted in the selection of six sites that ranged from just south of the two arms to slightly south of Bartlett Cove (fig. 2).

In 1997, these sites were sampled using both the FG and CG methodologies. The FG data from these sites have inference to the range of cobble/boulder habitat within the band or region that the sites occupy, as these sites also were part of the CG set of sites, which had been randomly selected from the pool of the selected habitat type. Thus, the FG sites are an approximately random sample of predominantly cobble/boulder habitat within the exact band they occupy.

The FG sampling consisted of multiple types of sampling (fig. 3), which are detailed in appendix A. I briefly describe four of the sampling types here: (1) vertical transect by point intercept; (2) horizontal transect by point intercept, (3) quadrat, by point intercept and counts; and (4) band survey by counts. All point-intercept methods targeted sessile species, but counted all species, sessile and mobile, under points. The first sampling type was point-intercept sampling along 10 vertical transects sampled at 5 points $/ \mathrm{m}$. The 3D point-intercept sampling methods were the same as those used in the CG sampling. The vertical transects were laid out in a similar manner as those in the CG sampling, except that the sampling of 10 transects led to a smaller (20-m) systematic distance between transects.

In the second sampling type, 30 horizontal transects were sampled per site. Each measured $10 \mathrm{~m}$ in length and they were arrayed three per vertical transect, with the location and elevation determined by random-systematic methods. The length of a vertical transect was divided into thirds and a random number identified the location within one of the zones where the horizontal transect origin would be laid. The other two horizontal transects were set at systematic intervals from the first within the two other vertical zones of the beach. Horizontal transects were sampled by point-intercept sampling (targeting sessile species) that used the same intensity ( 5 points $/ \mathrm{m}$ ) and sampling methodology as employed for vertical transects. 
In the third sampling method, a quadrat was sampled at each juncture of the vertical and horizontal transects, with 3 arrayed per vertical transect. This led to a total of 30 quadrats sampled per site. Quadrats were $1 / 9 \mathrm{~m}^{2}$ in area and contained a grid of 36 intersections at which points were sampled (fig. 4). Quadrat point-intercept sampling targeting sessile species used the same 3D methodology as used for vertical and horizontal transects. Additional sampling within quadrats included counts of small mobile invertebrates and subsampling counts of littorine snails and barnacle spat/recruits (fig. 4).

The fourth FG sampling method, band surveys, targeted large mobile invertebrates by sampling 1-m-wide bands on each side of the vertical and horizontal transect lines (fig. 3). Methods used were the same as described earlier in the CG sampling.

\section{Analytical Methods}

The analytical methods used to estimate the abundance of species/taxa, analyze trends for the predominant taxa, and estimate the power of different sampling schemes to detect changes in abundance for the these major taxa are described below.

\section{Estimating Abundance}

A general bay-wide measure of the abundance of species can be given by the total number of hits (counts) of a species across all sites sampled by the CG sampling. Within-year and 3-year (1998-2001) comparisons of the abundance of different species can be made because the total number of points sampled in any one year and for all years (the total) would be the same for all species.

Percent cover was the metric of abundance for sessile species at the transect and site levels. Percent cover can be calculated for all species sampled by point-intercept sampling. However, for the power analyses, which comprise the majority of the analyses presented, specific percent cover measures for each transect at each site were calculated for the predominant sessile species or species groups (also referred to as taxa):

1. The mussel, Mytilus trossulus,

2. The brown alga or rockweed, Fucus distichus subsp. evanescens (note: formerly known as Fucus gardneri), and

3. All barnacles (Balanus glandula, Semibalanus balanoides, Semibalanus cariosus, Chthamalus dalli, Balanomorpha, and barnacle spat/recruits). Note: all species were recorded to the lowest taxonomic level possible in the field, but for the purposes of analysis, all barnacle taxa and categories (for example, spat/recruits) were combined.

For each site, the percent cover was calculated by dividing the number of hits of the selected species on each transect, by the number of points counted along the transect. Because the counts were done in 3D and multiple layers of a species could be encountered at each point, the percent cover had the potential to exceed 100 percent. 
Due to differences in the intensity of point sampling between 1997 and all subsequent years, data analyses were performed on two different CG datasets: a 4-year (1997-2001) dataset and a 3-year (1998-2001) dataset. In 1997, vertical transects at all sites were sampled consistently at a minimum of 1 point $/ \mathrm{m}$, whereas in 1998-2001, sampling was conducted at 5 points $/ \mathrm{m}$. To maximize the temporal comparison of data from the 1997 to 2001 period, data from 1998 to 2001 were reduced such that only 1 point/m data were included in the 4-year (19972001) dataset. When the 1998-2001 datasets were reduced, the single point selected to be included was always that at the meter mark (for example, $1.0 \mathrm{~m}, 2.0 \mathrm{~m}$, etc.), which matched the sampling conducted in 1997. The 3-year (1998-2001) dataset, which used the full intensity (5 points $/ \mathrm{m}$ ) data, was used in a number of analyses, including those where the effect of variation in the intensity of point sampling on power was being examined.

\section{Trend Analyses}

Trend analyses for the predominant sessile taxa (barnacles, Mytilus and Fucus) were conducted by TerraStat Consulting Group (hereafter referred to as TerraStat; see appendix B for contact information). The 1998-2001 data for each species from each of the 25 sites were tested for exponential trends by fitting a linear regression to log-transformed abundance (percent cover) data. Site and regional trends were evaluated; regional is defined as Glacier Bay proper.

\section{Power Analyses}

MONITOR software (James P. Gibbs, 1995) was used to estimate the power (1- $\beta$ ) of CG and FG sampling, as well as the influence of a number of parameters on power. For multiple-site, multiple-year analyses, MONITOR uses a route regression approach to test for trends. For greater detail, see appendix B or Gibbs and Ramirez de Arellano (2007). I used a version of MONITOR with a corrected exponential model (modified MONITOR, sensu Hatch, 2003) in my analyses of the 1997-2001 data.

After I completed the analyses using the corrected exponential version of MONITOR, a general concern arose regarding assumptions used by the MONITOR program. To address this concern, I contracted with TerraStat to review and reanalyze a small, but key subset of CG 19982001 data. These included power analyses for the three major sessile taxa at 25 and 15 sites.

Described below are the methods I used to analyze the CG data, the FG data, and comparisons between the CG and FG data. These are followed by the methods used by TerraStat. 
In my use of MONITOR for multiyear CG data analyses, each site represented a 'plot' as defined in MONITOR guidance documents and all plots were weighted equally. I selected the exponential trend option, a constant coefficient of variation, and specified use of two-tailed ttests. The significance level (alpha, or $\alpha$ ) was set at 0.05 , except for those specific cases where alpha was varied to examine its effect on power. My runs of the program consisted of 500 iterations. The biological data used for the analyses were a mean ("plot count") and variance ("plot variance") measure for a species at a site for the time period under analysis, either the 3year $(1998,1999,2001)$ or 4-year $(1997,1998,1999,2001)$ periods. As described earlier, 3-year data were sampled at 5 points/m intensity, and 4-year data were reduced, for consistency, to 1 point $/ \mathrm{m}$ intensity. Details on why I split the data into different time periods were presented above and related to the reduced intensity of point sampling in 1997. The percent cover for each taxon was determined as described, prior to calculating the individual site means and variances needed for computing power in MONITOR.

Statistical analyses to calculate the "plot count" and "plot variance" required to run MONITOR were conducted using the software program StatView for Windows (version 4.57; Abacus Concepts, Inc., 1996). The percent cover along each transect for each of the predominant taxa at each site was imported from Microsoft ${ }^{\odot}$ Excel into StatView. The mean percent cover for a taxon at each site was an average of the percent cover calculated for the six transects over all 3 or 4 years, depending on the dataset used. This mean for a taxon at each site was the plot count used in MONITOR.

In my analyses, plot variance, the second variable required by MONITOR, was the residual mean-square-error of a linear regression on untransformed data (percent cover of a taxon for each transect at a site over time).

I examined the relative importance of different parameters on the power to detect changes in the abundance of the three major sessile taxa (table 1). The largest number of analyses used the CG data with $\alpha=0.05$. MONITOR results are presented graphically.

For some analyses, fitting a trend line to the calculated points enabled extrapolation of data to determine how increased sampling of the number of points per meter, number of transects, number of sites, etc. would increase the power of the monitoring protocol. In general, these extrapolations were more useful when calculated power was low. Data reductions also were made. For example, the 1998-2001 CG data allowed the effect of the number of sites, number of transects within a site, and intensity of point sampling to be assessed through reductions of the data, whereas the 1997-2001 CG data allowed the number of sites and transects to be reduced. Site and transect data reductions were made using the mean and standard error of the regression calculated from either the 1 point $/ \mathrm{m}$ data (1997-2001) or the 5 points $/ \mathrm{m}$ data (1998-2001). When the number of sites was reduced, I used one random subset of sites to estimate power.

Most CG analyses were conducted on similar parameter sets for all three predominant sessile taxa. However, in a few cases only Mytilus data were used, because prior analyses indicated that power results for Mytilus were intermediate between those for barnacles (higher) and Fucus (lower) (Irvine, 1998). Thus, the results for Mytilus should indicate general trends in how different parameters affect the estimation of statistical power. I chose to use only Mytilus data to examine effects of more complex combinations of variables (for example, number of sites, level of population change, and number of points sampled per meter). 
The MONITOR program also was used to analyze the 1997 FG sampling data. Although the program was designed primarily for looking at the variance of counts made through time (Thomas and Krebs, 1997; Hatch, 2003), when only 1 year of data is available for computing power analyses, then replicate sampling within a site acts as a proxy for temporal sampling (that is, each transect represents a count). Then the mean and variance used in the MONITOR program are computed using the within-site, within-year results of sampling. In my analyses of the 1997 FG sampling data, subsets of the samples were selected (systematically and/or randomly); means and variances for those data subsets were computed and then used in MONITOR. Parameters examined for the FG MONITOR runs were similar to those used in the 3- or 4-year CG sampling analyses (table 1). Many runs based on the 1997 data are reported in Irvine (1998).

Additionally, I compared the power of CG and FG sampling to detect changes in the abundance of species, based on vertical transect data, because this method was common to both sampling regimes. As the FG sampling was conducted only in 1997, I wanted to include the 1997 CG data in the comparisons. Therefore, FG data were compared to CG 1997 data or CG 19972001 data, all taken at 1 point $/ \mathrm{m}$ sampling intensity. Analyses were conducted for the three main sessile taxa across various sampling schemes that involved data from either the $6 \mathrm{FG} / \mathrm{CG}$ sites or the full set of 25 CG sites.

Effort was not standardized between the FG and CG sampling regimes. The closest comparison, with respect to effort, would be that for the FG and CG 1997 sampling conducted at the same six sites.

TerraStat performed power analyses on a subset of the 1998-2001 CG data using a more recent version of MONITOR (version 10.0; Gibbs and Ramirez de Arellano, 2007), as well as some different parameters (appendix B). The intent of the reanalyses was to determine if changes to the MONITOR program or changes to parameters used in the analyses caused large differences in estimated statistical power.

TerraStat compared three different analyses: (1) use of TerraStat parameters (see below) in MONITOR version 10.0; (2) use of my original parameters in MONITOR version 10.0; and (3) my original results, which had used my parameters in the corrected exponential version of MONITOR. Additionally, TerraStat examined the effect of the number of sites, by contrasting the power obtained by sampling 25 sites versus 15 sites.

The parameters used by TerraStat in its power analyses included: (a) plot means - the initial year's plot means; (b) plot variance - the standard error of the residuals from an exponential-regression fit to untransformed densities; (c) site reductions - power averaged over 10 random subsets of the 25 sites; and (d) number of iterations-1,000 (appendix B). 


\section{Results}

Obtaining 4 years of data has allowed: (a) assessment of the abundance of different taxa, (b) analysis of trends in the abundance of the predominant sessile taxa at a site and regionally, and (c) power analyses. Collectively, these analyses support the goals of detecting long-term trends in the abundance of taxa and determining the most appropriate methods for doing so.

\section{Abundance of Taxa}

Fucus, barnacles and Mytilus were much more abundant than the 78 other taxa encountered in the CG point-intercept sampling along vertical transects (table 2). In the sampling conducted in 1998-2001, of the 61,736 species hits (counts), Fucus was counted 19,773 times (32 percent), Mytilus 17,903 times (29 percent), and all barnacles 16,047 times (26 percent). These three taxa accounted for 82 percent of all species hits. The next most abundant grouping of taxa, all green algae, comprised 3,603 hits (5.8 percent). This disparity in relative abundance led to the focus on the three most abundant sessile taxa for subsequent analyses.

\section{Trend Analyses}

There was considerable variability in the abundance of the predominant sessile taxa, both spatially and temporally (1998-2001), which affected the ability to detect site and regional trends (tables 3-5; appendix B). No significant regional trends were determined for Mytilus or Fucus. Only barnacles had a significant regional trend (mean trend $=+22.5$ percent; 2 -tailed t-test, $\mathrm{p}$ value $=0.0095$; appendix B). For barnacles, all significant trends at individual sites were positive (seven sites), whereas significant trends at sites were more mixed in sign for Mytilus (three positive, five negative) and Fucus (four positive, one negative) (tables 3-5). The largest range in magnitude of trends among sites occurred for Fucus $(-70$ percent per year to +220 percent per year, appendix B). Further results and details of trend analyses are presented in appendix B.

\section{Power Analyses}

I present my power analyses results for: FG sampling, CG sampling, and comparisons of the CG and FG sampling. All these analyses were conducted with $\alpha=0.05$, except for those specified CG analyses where alpha level was varied. These are followed by results of the TerraStat reanalyses, which include comparisons to some of my results.

\section{Fine-Grained Sampling}

The FG data were used to evaluate the power of different point-intercept sampling methods (vertical transects, horizontal transects, and quadrats) to detect change in the abundance of sessile species. Across species, vertical transect sampling provided the greatest power to detect change, generally followed by horizontal transect sampling, then quadrat sampling (fig. 5). 
The power to detect change varied consistently among the three main sessile taxa, with the greatest power for barnacles, somewhat less power for Mytilus, and least for Fucus (fig. 5). The only species group for which the FG sampling of six sites provided sufficient power $(>0.8)$ was barnacles; for this group, the vertical transect sampling produced the highest power $(>0.9)$ (fig. 5C). The number of vertical transects needed to detect 10 percent decreases in Mytilus and Fucus at 0.8 power, when only six sites are sampled, was estimated to be 20 and 35 transects, respectively (fig. 6); these numbers are 2-3.5 times higher than the 10 vertical transects sampled under the FG regimen.

Additional analyses of the 1997 FG data that examined the combined influence on power of varying the number of vertical transects and number of points per meter sampled, indicated that increasing both the number of transects and the number of points sampled per meter generally increases power. Only for Fucus, where power was low and variable, were some of the extrapolated relationships not as consistent as for Mytilus and barnacles (appendix C, figs. $\mathrm{C} 1-$ C7).

\section{Coarse-Grained Sampling, Alpha $=0.05$}

Analyses of the 3- and 4-year CG datasets allowed comparison of the effect of the number of years versus the intensity of point sampling on power. The power of the CG sampling of 25 sites, conducted over 4 years (1997-2001) at 1 point/m intensity (fig. 7), was very similar to that found for analyses of the 3-year (1998-2001) CG data taken at 5 points/m intensity (fig. 8 ). This indicates that increased temporal sampling can compensate for decreased intensity of point sampling.

I found that attempting to increase power by increasing the number of points sampled per meter is not very efficient. The ability to detect 10 percent decreases changes little as CG sampling is increased from 1 to 5 points/m, and remains high for all three taxa (figs. 9-11). In most of the 5 percent and 3 percent annual change graphs (figs. 9-11), the power lines are fairly flat or gently sloping, except for Mytilus. At any given sampling intensity, the power is greater for barnacles and Mytilus, and lesser for Fucus. If the minimum goal is to have a power of at least 0.8 to detect a 10 percent annual change in abundance, then a sampling intensity of 1 point/m in the CG sampling is sufficient for barnacles and Mytilus. For barnacles and Mytilus, there is very high power $(>0.98)$ to detect 10 percent annual changes, even at a sampling intensity of 1 point $/ \mathrm{m}$.

Increasing the number of transects increases power, as was demonstrated by analyses of the CG (1998-2001) data for Mytilus (fig. 12). Only one transect/site is needed to achieve a power of 0.8 to detect a 10 percent decrease in Mytilus. At two transects/site the power to detect a 10 percent annual change is $>0.9$ (fig. $12 \mathrm{~A}$ ). With six transects/site, the power to detect a 5 percent annual change is 0.8 (fig. 12B).

Increasing the number of sites sampled increases the power to detect changes in Mytilus abundance, as illustrated by analysis of CG 1997-2001 data (fig. 13). Sampling 25 sites provides a 1.0 probability of detecting a decrease of 10 percent per year. In fact, sampling 10 or more sites per year produced a power of $\geq 0.9$ to detect 10 percent decreases in Mytilus (fig. 13A). A 5 percent decrease at 0.8 power can be detected by the CG sampling of 25 sites (fig. $13 B$ ). 
When both the number of sites and the number of points per meter sampled are increased, the power to detect trends in Mytilus abundance generally increases (fig. 14). Analyses of the 1998-2001 CG data indicated that a $>90$ percent probability ( 0.9 power $)$ to detect a 10 percent decrease in Mytilus was readily accomplished by sampling $\geq 15$ sites at 1 or more points per meter (fig. 14A). Detecting a 5 percent decrease with 0.9 power would necessitate sampling more points per meter: for 25 sites, about 6 points $/ \mathrm{m}$; for 20 sites, about 8 points $/ \mathrm{m}$ (fig. 14B). Detecting a 5 percent decrease at a power of 0.8 can be accomplished by sampling 25 sites at about 3 points $/ \mathrm{m}, 20$ sites at about 6 points $/ \mathrm{m}$, or 15 sites at about 9 points $/ \mathrm{m}$.

\section{Coarse-Grained Sampling, Varying Alpha}

When the accepted error level (alpha) is increased, the power to detect changes in the abundance of the predominant taxa increases (fig. 15). Analysis of the 1997-2001 CG data indicated that power was high $(\geq 0.9)$ to detect 10 percent changes in the abundance of Mytilus and barnacles at a range of alphas $(0.05-0.20)$. The power to detect changes in abundance of Fucus generally was lower than for the other taxa. A 0.8 probability of detecting a 10 percent change in Fucus generally required $\alpha \geq 0.10$, except for a negative 10 percent change where $\alpha=$ 0.05 sufficed (fig. 15). Detecting 5 percent decreases in all three taxa at a power of 0.8 or greater requires $\alpha \geq 0.20$ (fig. 15).

Additional analyses of the effects on power of varying alpha in combination with other parameters support the increase in power when alpha is increased along with increases in number of transects; lesser effects on power occur when alpha and the number of points sampled per meter are increased (appendix C, figs. C8-C26).

\section{Comparison of Coarse-Grained and Fine-Grained Sampling}

The power of the CG and FG sampling plans varies for the different species, but the CG sampling of 25 sites is more powerful than the FG sampling of 6 sites (fig. 16). There was less difference between these two sampling schemes for barnacles, which was the only taxon that was adequately sampled (power $>0.8$ ) by the FG sampling. For mussels and Fucus, however, the CG sampling at 25 sites was distinctly more powerful (fig. 16).

Comparisons of various sampling plans using only 1997 data indicate that CG sampling of 25 sites has more power than the FG sampling of 6 sites, which in turn has more power than the CG sampling of 6 sites. These results for 1997 data support the positive influence on power of increasing the number of sites or the intensity of sampling. In particular, note that the 1-year CG sampling of 25 sites has more power than the 1-year FG sampling of 6 sites (fig. 16).

The most equivalent comparison of FG and CG effort and power is comparison of the 1997 data for the 6-site analyses (fig. 16). These analyses are for data from the same set of sites. Effort is somewhat greater for the FG sampling (10 transects/site sampled at 5 points $/ \mathrm{m}$ ) versus the CG sampling ( 6 transects/site sampled at $1 \mathrm{point} / \mathrm{m}$ ), and the difference in power reflects that increased effort. There is no equivalent comparison of effort and power for the 25 -site CG sampling versus the 6-site FG sampling; effort is much greater for the 25-site CG sampling. 
The multispecies results, condensed in figure 17, compare the relative power of the CG (1997-2001) sampling and the FG (1997) sampling to detect changes in abundance. For all taxa, the multiyear CG sampling of 25 sites has more power to detect change. These results reflect the positive effects on power of increasing the number of sites sampled or temporal sampling.

\section{TerraStat Power Reanalyses}

The TerraStat results for barnacles and Mytilus were quite similar to those I obtained in previous analyses (figs. 18, 19, 21), including analyses for Mytilus that examined the effect of number of sites on power (figs. 18 and 19). However, TerraStat results for Fucus indicated a decrease of 5-10 percent in power to detect changes in its abundance (fig. 20 and appendix B). This decrease in power for Fucus appeared to be due primarily to the differences in starting parameters used rather than to changes to the MONITOR program, because the starting mean for Fucus in the new analysis was an average of 4 percent lower than the values I used (appendix B). Note that the results of my original analyses shown in figure 20 are aligned with reanalyses that used my original parameters in the newer MONITOR 10.0. This also indicates that it is a change in the parameters used for the TerraStat Fucus analyses, rather than the changes in the MONITOR program that caused the differences in power results for Fucus. The similarity in power results for barnacles and mussels when both MONITOR 10.0 and its earlier version are used, and when both TerraStat and my parameters are used, provide support for being able to directly interpret prior analytical results for those species groups. The TerraStat findings of somewhat reduced power to detect change in Fucus abundance should be taken into account when interpreting my earlier analyses. Implications of this finding for long-term monitoring design are examined in section, "Discussion."

TerraStat analyses that manipulated the number of sites found that decreasing the number of sites sampled from 25 to 15 decreases the power to detect trends for the three predominant sessile taxa (figs. 18-21). For Mytilus and barnacles, this change is minimal at the 10 percent trend detection level, but becomes more important for detection of smaller annual trends (figs. 18, 19, 21). However, for Fucus, the TerraStat analyses indicated lesser ability to detect trends: even with 25 sites sampled, the ability to detect 10 percent trends was below the 0.8 power level specified as the minimum desired level, although the ability to detect negative 10 percent decreases almost reached the 0.8 level (fig. 20). When only 15 sites are sampled, the power to detect 10 percent annual trends for Fucus significantly decreased (fig. 20).

\section{Discussion}

During the Exxon Valdez oil spill in 1989, extensive intertidal habitats and their resident biological communities were affected directly by oil. In the injury assessment following the spill, great emphasis was placed on statistically sound sampling designs that allowed results to be more broadly generalized, and on the need to sample intensively enough to detect effects. As a result, probability-based designs were used in some studies (for example, McDonald and others, 1995; Peterson and others, 2001). This setting, coupled with the recognition that the NPS lacked baseline data for the biological resources on its coasts, stimulated the interest of the NPS in developing probability-based designs for long-term monitoring of intertidal communities along national-park coasts. 


\section{Study Design}

One objective of monitoring is to detect change in populations or communities through time. However, the ability to detect change is related to the extent of the variation that exists, and the design and intensity of sampling. Managers of expansive national parks, such as Glacier Bay NP\&P, are faced with what seem to be daunting challenges imposed by the tremendous physical scale and remoteness of the environments that they manage.

An approach to reconciling these different issues is through use of probability-based designs. Such designs allow the results of surveys to be extrapolated to the universe of sites from which the sampled sites were selected (the sampling frame). Deviations from the design cause some reduction in the extent or scope of inference.

A central purpose of this project was to provide managers with an analysis of several probability-based monitoring designs conducted at different scales of inference and effort. The three levels of survey and sampling included aerial surveys, CG sampling of many sites, and FG sampling of a few sites. An adaptive-sampling approach was used, where results of the first stage of surveys informed the decision making that led to the next sampling stage, etc.

The first stage, the aerial surveys, characterized the frequency of different intertidal substrate types within Glacier Bay. Of the 250 segments identified, 9 could not be surveyed, thus slightly reducing the extent of inference to the universe of coastal segments delineated within Glacier Bay proper. The systematically selected set of characterized segments also is available for other studies, thus facilitating further establishment of probability-based studies in Glacier Bay. This pool of sites has since been used by researchers examining the effects of sea otters on intertidal clams (Bodkin and others, 2007).

The results of the aerial surveys led to a revision in the characteristics of habitat types selected for the monitoring program. The finding that bedrock-dominated habitat with slope $\leq$ $60^{\circ}$ was rare was unanticipated, even by resident NPS staff. The abundance of cobble/boulder habitat, and its location in the more protected waters of Glacier Bay where it is likely to be stable, led to its inclusion along with bedrock as the habitats of focus (Irvine, 1998). The unexpected results of the aerial surveys illustrate one of the values of probability-based surveys for avoiding bias.

At the CG sampling level, 5 of the 30 randomly selected sites could not be sampled, for reasons given in section, "Methods." The elimination of these five sites somewhat reduces the extent of inference of the sampled CG sites to what was defined as target habitat within Glacier Bay. Except for the site eliminated because it was in a wildlife protection area, these eliminations may provide an estimate of the error of the segmentation process and the aerial surveys in correctly describing intertidal habitat within Glacier Bay.

The error rates can be estimated for both the aerial surveys and the selection of CG sites. During the aerial surveys, 9 (or 3.6 percent) of the 250 segments selected were flown but not surveyed. The reasons for not surveying a segment included: (a) segments actually were stream banks and not intertidal habitat $(n=4)$, which likely is an error arising from the digitization of the coastline; (b) segments were snow covered at the time of the survey $(n=3)$, therefore could not be surveyed; and (c) segments apparently had changed since the charts used for the digitized coastline were created. Segments in this latter case occurred in areas of rapid sedimentation and, despite their coordinate matches, did not resemble the ArcInfo segment maps. The snow-covered segments also might have had a slightly higher elevation, because the tides had not removed the snow. 
During the selection of CG sites, 5 of 30 randomly selected segments were eliminated because they were in a wildlife protection area, too steep, or not a true beach (had no land exposed at high tide). After subtracting the one site that was in a wildlife protection area, these four eliminated segments constituted 13.33 percent of those selected. These represented errors in the classification process, but because all were surveyed at low tide, it would have been difficult during the surveys to determine those that were not true beaches.

The CG and FG sampling plans were designed to examine the ability to effectively sample the variation expressed in intertidal communities across different scales. The scales reflected geographic variation and within-site (local) variation. These variations were addressed, respectively, by increasing the number of sites sampled and their geographic scope, and by increasing the intensity of sampling. Geographic inference differed for the CG and FG sampling plans, due to the selection of FG sites from a narrower band of Glacier Bay (approximately the lower half of the bay). The decision to sample cobble/boulder-dominated sites during the FG sampling reduced the inference to that habitat within the narrower geographic band. Although I found some decrease in power to detect change in Mytilus geographically, based on analyses of 1-year data (Irvine, 1998), I have not conducted similar analyses for other intertidal species nor on multiyear data. Further analyses might indicate if a geographical bias exists.

A major question posed in this study was whether sampling many sites less intensively (CG sampling of 25 sites) had higher power to detect trends than did more intensive sampling of fewer sites (FG sampling of 6 sites). I found that the CG sampling had greater power to detect changes in the abundance of the three predominant sessile taxa.

For within-site sampling that targeted sessile taxa, vertical transects were selected as a sampling technique for several reasons. First, they sample across the elevational zonation of species, which is one of the major sources of variation in intertidal communities. Second, if climate change occurs, then the vertical distribution of species could be affected. For example, zones could shift vertically without the relative abundance of a species changing across a beach. If fixed quadrats or horizontal transects initially are established within zones, then changes noted in the abundance of a species or an assemblage might reflect changes for that position, but might not reflect changes occurring across the beach. Vertical transects also provide a good approach for sampling cobble/boulder habitat, as the draped transect lines sample topographically diverse substrates better than quadrats, due to the complication of variation in surface area sampled within quadrats. In addition to these rationales for using vertical transects, I found that sampling of vertical transects has greater power to detect changes in abundance of the major sessile taxa compared to the other point-intercept methods tested (quadrats and horizontal transects; fig. 5). Further support for the usefulness of vertical transects comes from a study on bedrock substrates in southern California that found that sampling of randomly placed vertical transects more precisely estimated the percent cover of species than did fixed plots (Miller and Ambrose, 2000).

The effort and cost associated with the CG and FG sampling regimes is not equivalent, as could be expected from the large difference in the number of sites sampled. This is discussed in section, "Design Issues for Glacier Bay Intertidal Monitoring." 


\section{Comparison with Other Intertidal Monitoring Designs}

For this study, a probability-based monitoring design was planned to test whether the variation that exists in rocky-intertidal assemblages in Glacier Bay could be sampled sufficiently to allow trend detection of the predominant sessile species, while providing inference to the universe of the selected habitat type within the bay. The goal was to provide managers with the ability to broadly generalize changes in abundance. Most sampling of rocky intertidal environments has not followed a probability-based monitoring approach, but has relied on selected sites (Channel Islands National Park (NP) - Richards and Davis, 1988; Olympic NP; Kinnetic Laboratories, Inc., 1992; Partnership for Interdisciplinary Study of Coastal Oceans [PISCO]; and Multi-Agency Rocky Intertidal Network [MARINe] — Ambrose and others, 1995; Raimondi and others, 1999) and often fixed plots or transects.

The population approach taken by Channel Islands NP staff led to target species being followed in fixed plots at selected sites (Richard and Davis, 1988). The intent was that the results would provide trend information for those species, as well as for early detection of abnormal conditions. The MARINe program primarily has sites throughout California, but in connection with PISCO has stretched geographically to include some sites in Mexico, the Pacific Northwest (including British Columbia) and Alaska. Sites are selected and intentionally spaced, and the core protocol targets specific algal and invertebrate assemblages. However, the use of vertical transects and some randomization in their placement allows sampling to be extrapolated to the site. Biodiversity sampling at the site provides data with great taxonomic definition. Band surveys and quadrat sampling also are conducted at sites. The creation of a broader network of sites allows the detection of regional and latitudinal biogeographical changes that may result from broader-scale effects due to oceanographic or climate change. Changes to sites from oil spills or other effectors can then be understood within a broader context (P.T. Raimondi, University of California, Santa Cruz, pers. comm., 2008).

Selecting sites, quadrats or transect locations without a random component to the choice limits the ability to make broader inference from the results. When sites, or other sampling units, are selected to be similar, then it is likely that variation would be reduced. If so, then the power to detect change should be increased. The growth in development of sampling designs for impact and monitoring studies is discussed in Schmitt and Osenberg (1996) and Murray and others (2006).

Two additional probability-based designs for intertidal monitoring are in progress in Alaska. One is an offshoot of the Glacier Bay design, which I adapted for Sitka National Historical Park in southeast Alaska. Sitka has a short coastline (approximately $1 \mathrm{~km}$ ), so the whole beach is defined as the sampling frame. Sessile species are being sampled by vertical transects set systematically with a random start; large mobile invertebrates currently are sampled by band surveys along the vertical transects; and small mobile invertebrates are sampled in quadrats placed systematically along the transects. Power analyses were conducted to test whether the design can detect desired levels of change in target species (Irvine and Madison, 2008). 
Since the Glacier Bay and Sitka intertidal monitoring projects were initiated, another probability-based approach has been developed, the generalized random-tessellation stratified (GRTS) survey design (McDonald, 2004; Stevens and Olsen, 2004). This approach creates a spatially balanced sample and has flexibility for adding sampling units while maintaining the spatial balance. Thus, it improves upon both systematic and simple random designs. The only intertidal monitoring project I am aware of that is using this approach is that of the Southwest Alaska Network of the National Park Service (Bodkin and others, 2008). This project is currently in the design and testing phase.

\section{Power, Trend Detection, and Impact Analysis}

Power is the ability to detect a trend if one is, in fact, occurring (or as stated earlier, the probability of rejecting the null hypothesis when it is false and should be rejected). A priori power analyses are used during the monitoring design phase to estimate how effective the monitoring plan is likely to be. Because these analyses often used limited temporal data, they should not be viewed as being predictive, just the best estimate of how various designs will perform.

The levels that are set for power and other aspects of the monitoring design vary amongst programs. Channel Islands NP has set, as their goal for monitoring, a power of 0.8 to detect a 40 percent change in species abundance through time, with an alpha of 0.05 . It is not clear over what time frame this 40 percent change is computed. The North American Amphibian Monitoring Program (http://www.pwrc.usgs.gov/naamp/) previously stated on their website that monitoring programs should be able to detect population trends with a power of 0.9 , but an alpha of 0.20 . Their rationale for setting a relatively high alpha value is that it is more important, from a conservation standpoint, to detect declines than to be correct about whether they are occurring (Sam Droege, U.S. Geological Survey, written comm., 2009). The signaling of a decline could initiate further sampling or research to clarify the trend or investigate causes. For threatened or declining species, however, failing to detect a trend could be detrimental to the species and to management, therefore setting a smaller error rate (for example, $\alpha=0.01$ ) may be an important aspect of the monitoring design (Gibbs and Ramirez de Arellano, 2007).

Setting the levels of change to be detected is a management decision, but it should be made with cognizance of how short-term changes compound over time. Even small annual changes of 5 percent quickly accelerate over time to appreciable levels of change (table 6).

Various types of power analyses can be performed. I have focused on comparing the ability of different sampling strategies to detect trends in the predominant sessile species through use of the MONITOR program. Trend detection is the goal of most monitoring programs so the power to detect trends in counts or abundance is assessed frequently. Another type of power analysis has focused on the ability to assess impacts, and uses power analysis to compare one site before and after impact (Before-After sampling design) or comparisons of two or more sites (Before-After Control Impact [BACI], or Before-After-Control-Impact Paired [BACIP] designs) (for example, Schroeter and others, 1993; Osenberg and others, 1996; Minchinton and Raimondi, 2001). Additional permutations of this impact type of sampling design have been suggested (for example, Stewart-Oaten and others, 1986; Underwood, 1994). These types of analyses could be performed on Glacier Bay data if there were suspected or known effects to one or a group of sites. Having a number of sites which could act as controls for an affected site, with both categories followed for multiple sampling intervals both before and after an event (an asymmetrical BACI design, Underwood, 1994) might provide the best option for assessing the degree of impact to that one site. Thus, the data gathered through time from the network of sites provides a backdrop against which impacts can be assessed. 


\section{Variation in the Ability to Detect Change among Taxa}

Power to detect change varied consistently among the three predominant sessile taxa. Highest power to detect change occurred for barnacles, followed by lower power for the mussel Mytilus trossulus, then the rockweed Fucus distichus subsp. evanescens. Power will be related inversely to variability and/or abundance.

Power analyses of Channel Islands (California) rocky intertidal data also indicate differences among taxa, with greatest power to detect changes for rockweeds, lower power for the California mussel Mytilus californianus, then barnacles, and lowest power for Endocladia muricata, a red alga (Minchinton and Raimondi, 2001). Because the species comprising these taxonomic groups, as well as their abundances and ecology, differ from those in Glacier Bay, it is not surprising that the power relationships among taxa are not the same. For example, the rockweeds are comprised of entirely different taxa: Silvetia compressa (formerly Pelvetia fastigiata) and Hesperophycus californicus (formerly H. harveyanus) (see MARINe website: http://www.marine.gov/Research/CoreSurveys/SeaweedOfCalifornia.html). The Glacier Bay sites also are protected, while the Channel Island sites are exposed rocky (bedrock) habitat. The study design also influences the power results; because many of the plots at Channel Islands were selected to be within zones dominated by these target taxa (Richards and Davis, 1988), the abundances of the target taxa generally are fairly high. The geographical variation in power for similar taxa (for example, rockweeds) indicates that we should use caution when borrowing information from other areas to guide power analyses.

There is some indication from Glacier Bay data that greater variation in the abundance of a species (only Mytilus data for 1997 were examined) led to decreased power. The trend in power was related to geographical distribution of sites, with decreased power to detect change in Mytilus for sites lower in the bay (Irvine, 1998). This decreased power may be related to the increased species diversity found in the lower part of the bay (Sharman, 1990).

The geographical trend in the power to detect change in Mytilus abundance calls to the fore another issue, which is that the abundances of the major space-holding species in the intertidal are not independent. Competition for space occurs, and can be mediated by predation and disturbance (for example, Connell, 1961; Paine, 1966; Dayton, 1971; Sousa, 1979). Thus, variation in one species can affect variation in other species. If additional species become more common in Glacier Bay as glaciers recede or as climate warms, then the relative abundances, and hence power to detect change in barnacles, Mytilus, and Fucus may change. Because this sampling program is not targeting specific species, but is assessing the biotic composition of a site, differences in the occurrence and relative abundance of species through time will be assessed. The species that are dominant now may not be dominant at some time in the future. Sampling at the CG level may make it difficult to detect species with low abundances, however, and could be combined periodically with more intensive sampling, site surveys, or timed surveys to increase detection of less-common species. 


\section{Design Issues for Glacier Bay Intertidal Monitoring}

The power and design of a monitoring program will be influenced by both managementset parameters (for example, the desired power, level of change to be detected, and alpha level), and sampling parameters (for example, number of sites, transects, quadrats, etc.). The biological data provide the basic information needed for assessing how to sample to achieve the management-set parameters. Another factor greatly affecting the ultimate sampling design is cost. I consider first how different designs (for example, CG and FG) and parameters affect power, then discuss effort and cost considerations.

CG sampling provides more power to detect change than the FG sampling, which is due primarily to the increase in the number of sites sampled under the CG regime. Increasing the number of sites, number of transects, or intensity of points sampled per meter all increase the power to detect trends. The effect of increasing sites and transects is pronounced, generally more so than increasing the intensity of point sampling. It may be harder to observe the effect of increasing point sampling, as very low-intensity point sampling has high power to detect change for barnacles and Mytilus. However, the power curves associated with increasing point sampling for barnacles and Fucus have fairly flat or gentle slopes, indicating that increasing the number of points sampled per meter has little influence on power.

Manipulations of alpha (error level) had relatively straightforward effects on power, which were attenuated when power reached very high levels (for example, 1.0; fig. 15). Increasing alpha increased the ability to detect trends, but, in general, the effect seems to be somewhat less than the effect of increasing the number of sites (fig. 15 versus figs. 18-21).

How do the CG and FG sampling differ in effort and cost? I stated in the "Results" section that the most equivalent comparison of effort and power between the CG and FG sampling is rendered by comparing the power analyses of the same six sites sampled by each method in 1997 (fig. 16). The increased intensity of the FG sampling led to somewhat increased power to detect change for all three of the predominant sessile taxa, with the greatest increase in power for barnacles. If we examine the effect of increasing the number of sites on power by comparing the analyses of CG data (1997, 6-site data versus 1997, 25-site data; fig. 16), we see a solid increase in power for all three sessile taxa. Multiyear sampling of the $25 \mathrm{CG}$ sites leads to further increases in power (fig. 16).

The major difference in cost or effort between the CG and FG sampling is exacted by the logistical cost associated with sampling more sites. Originally I had planned on sampling two CG sites per tide, but the distances between sites meant that only some site pairs could be sampled consistently on one tide (probably five pairs; although the exact number depends on the number of people sampling and their expertise). If we compare the FG and CG vertical transect/band sampling in terms of people-days, the FG sampling costs 18 people-days ( 3 people $\times 1$ site/day $\times 6$ sites), and the CG sampling costs 40-60 people-days (minimum 2 people $\times 0.8-1$ site/day $\times 25$ sites $=40-50$ people-days). Having three people doing the CG sampling increases the likelihood of being able to sample two sites per day, but the overall number of people-days would be 60. Thus, the CG sampling is approximately 2-3 times as expensive, in people-days, as the FG sampling, but samples 4 times as many sites. In the upper reaches of Glacier Bay, it is very helpful to have the support of a larger vessel than a skiff, but that further increases the costs. 
I considered setting initial conditions high: power of 0.9 to detect a 10 percent annual change in the abundance of the predominant taxa, with an alpha of 0.05 (Irvine, 1998). However, the reanalysis by TerraStat supports lowering the power level to 0.8 , or perhaps even lower for Fucus. A power level of 0.8 would encompass annual changes of 5-10 percent in Mytilus and barnacles sampled at 25 sites (figs. 18 and 21), 10 percent annual changes in Mytilus and barnacles sampled at 15 sites (figs. 19 and 21), and would come close to being able to detect 10 percent decreases in Fucus sampled at 25 sites (fig. 20). However, the power to detect 10 percent increases in Fucus is less, approximately 0.68 (fig. 20). This decrease in power from previous analyses is attributed primarily to the reduced plot mean estimate used for Fucus in the new analyses (average decrease of 4 percent in starting means; appendix B). The new analyses used the starting year mean values at each site as the plot or site mean input to MONITOR, whereas, my previous analyses used an average value for a taxon over all years. Increased temporal sampling is likely to increase the power to detect trends in Fucus. This is illustrated by the finding of very similar power for the 4-year (1997-2001, 1 point $/ \mathrm{m})$ and 3-year (1998-2001, 5 points $/ \mathrm{m}$ ) datasets (figs. 7 and 8 , respectively); in this case increased temporal sampling compensated for lower sampling intensity.

In addition to increasing temporal sampling, there are other options for increasing the power to detect change in Fucus. One would be increasing the number of sites, which provides the greatest increase in power (fig. 20). Increasing the number of transects sampled/site or the error level (alpha) also enhance power, but to a lesser degree. Increasing the number of points sampled/m has a yet smaller effect on power. Because the CG sampling, as conducted from1998 through 2001, provides high power to detect 10 percent annual changes in Mytilus and barnacles, no adjustments are needed to the CG sampling plan to effectively sample those taxa.

An alternative method for increasing power would be to make the vertical transects at the sites fixed in location, thus reducing the spatial variability over time. The data thus far have been from new (re-randomized) transect locations at a site each year. Because the power to detect trends for the major sessile species is relatively high, the challenges of marking and relocating precise transect locations may not warrant the effort. If quadrat sampling for small mobile invertebrates is coupled with vertical transect sampling in the future, then it probably would be worthwhile to fix the location of transects and quadrats to reduce the spatial component of variation in abundance due to re-randomization of sampling locations within a site each year. The abundance of small mobile invertebrates is likely more sensitive to changes in substrate composition.

If we assume that increased data collection over time would increase the power to detect change for Fucus, then the recommended sampling protocol for these sessile intertidal assemblages is to conduct the CG sampling scheme annually at the 5 points $/ \mathrm{m}$ level. The suggested protocol is detailed in appendix D. Because the newer power analyses indicate somewhat reduced ability to detect change in Fucus (fig. 20), I do not recommend reducing the number of sites sampled. Power analyses of the FG data provide an example of the repercussions on sampling intensity that result when only a small number of sites are sampled. In order to achieve the same 0.8 level of power to detect 10 percent decreases in the main sessile taxa, the number of transects per site would need to be increased to approximately 35 for Fucus and 20 for Mytilus when only 6 sites are sampled; current sampling is sufficient for barnacles (fig. 6). 
Although an equivalent level of power might be achieved by sampling fewer sites more intensely, there is additional value to having a larger number and greater spread of sites throughout the bay than was the case for the FG sampling. Note, however, that a random selection of a few sites from the entire bay might have good geographic spread and broad inference, but would not necessarily increase the probability of detecting different events. The logistic costs of visiting a few, quite dispersed sites also could be very high or prohibitive.

Sampling a larger number of sites is likely to increase the capture of particular types of effects, events, and changes, as well as increasing the likelihood that these sites better reflect the overall condition of the bay. If effects do occur that are likely to affect a small subset of the sites (for example, ice scour or trampling effects), having a larger number of sites increases the ability to compare affected versus unaffected sites. For example, site 69 (Berg Bay, fig. 1) was noticeably different from other sites in 1997 in being dominated by barnacles. I hypothesized that the site had been disturbed (probably by ice) prior to the spring set of barnacles in 1997. Later I learned from crab researchers that much of Berg Bay froze in the winter of 1996-97 (Tom Shirley, Texas A\&M University, and Jim Taggart, U.S. Geological Survey, oral commun.). The pattern of species abundances since then (fig. 22) is giving us a trajectory of the rate and pattern of secondary succession following a major site disturbance. Additionally, the slow recovery of Fucus, as compared to barnacles and mussels, indicates that it might be an important indicator of disturbance or change to intertidal communities.

Results detailed in this report indicate that generally high power exists to detect trends in the major space-dominating sessile taxa. As more temporal data are acquired, it is likely that the power to detect changes in trends of these sessile taxa will increase (for example, CG data, 25 sites, 1997 versus 1997-2001 data, fig. 16). An increase in power with additional data and increased temporal spread of data indicates that, in the future, the park could evaluate whether to sample these 25 sites every other year (or less frequently), or perhaps combine sampling the 25 sites every other year with sampling a subset in the intervening years for some indication of annual changes or major events.

This report has concentrated on analyses of sampling to detect trends in the major sessile taxa. For more complete sampling of intertidal assemblages, protocols are needed for sampling small mobile invertebrates (such as predatory snails, limpets, and littorine snails), and more effective sampling of large mobile invertebrates. Some data exist for both of these groups that could assist in proposing more effective sampling. Small mobile invertebrates were sampled within quadrats during the FG sampling. Large mobile invertebrates were sampled via band surveys along the vertical transects in both CG and FG sampling, but were uncommon. Sampling should be modified to concentrate sampling for this latter group (notably starfish) on the lower areas of the intertidal (Irvine and Madison, 2008).

Small and large mobile-invertebrate species can have dramatic effects on the abundances of the major sessile species under natural conditions (e.g. Irvine, pers. obs.), and following oil spills (Cubit and Connor, 1993; Highsmith, and others, 1996; Irvine, 2000). Therefore, development of protocols to effectively sample these groups would greatly strengthen an intertidal monitoring program and increase understanding of changes occurring to these communities. 
The sampling protocol as designed thus far has the power, if implemented and carefully executed, to enable the park to assess changes occurring to the major sessile species in these intertidal communities throughout Glacier Bay over the long term. To achieve and further this goal, I provide the following comments based on this study:

Implementation of the CG monitoring protocol for sessile species, as conducted from 1998-2001 (that is, sampling of 25 sites, 6 vertical transects/site, 5 points $/ \mathrm{m}$ ) will allow the park to detect 10 percent annual changes in the abundance of barnacles and Mytilus. Annual sampling will increase the ability to detect events, but sampling could be conducted at greater time intervals (for example, biennially or triennially).

The somewhat reduced ability to detect trends for Fucus argues against reducing the number of sites; however, increasing the time series of data is likely to increase the power to detect trends for Fucus.

If cost is an issue, the number of sites might be reduced, but this would reduce power to detect change in abundance of Fucus. If the number of sites sampled is reduced, the number of transects, points sampled per meter, etc. would need to be increased to maintain or increase power.

There are advantages to maximizing the number of sites sampled, while maintaining a random selection of sites. A GRTS design would allow greater flexibility for altering the number of sites while maintaining spatial balance of the sites. However, shifting to a GRTS design would result in a loss of applicability of many of the results of this study and the ability to extend the timeline of data from sites already established.

To more completely sample the macrobiota of the rocky intertidal communities within Glacier Bay, development of effective monitoring protocols for small mobile invertebrates and large mobile invertebrates should be considered.

\section{Why Monitor the Intertidal?}

The data obtained indicate that we can monitor the major sessile intertidal taxa. Why should we? Intertidal communities are very productive and diverse assemblages of species with extensive ties to terrestrial and marine species. The complexity and strength of the links has been illustrated by oil spills and a wealth of observation and investigation. The particular sessile species focused on here are all spatial dominants over broad geographical extents and are significant ecologically. Mussels and rockweed provide structure to the intertidal that can increase the species diversity of the communities. Additionally, mussels are an extremely important prey for many species including other invertebrates, fishes, birds, and mammals (including humans). Immigrating sea otters are expected to have effects on both intertidal and shallow subtidal species, including mussels, as their numbers continue to increase. Benthicfeeding waterfowl (for example, scoters [Melanitta spp.], goldeneyes [Bucephala spp.], and harlequin ducks [Histrionicus histrionicus) currently may be having a more extensive effect than sea otters. Surveys of marine predators in Glacier Bay have estimated that these benthic-feeding waterfowl have a combined population size greater than 30,000 individuals, with seasonal shifts in the abundance of different groups (Drew and others, 2008). Barnacles, another important prey for a number of species, also are included in the diets of black bears (Ursus americanus) in Glacier Bay and brown bears (U. arctos) in Katmai NP\&P. These intertidal communities, located at the juncture of the atmosphere, ocean and land are vulnerable to effects from all three directions. 
Monitoring of these rocky intertidal habitats falls under the mantle of long-term ecological monitoring. Although the direct relationship of the findings to management actions may not always be clear (Oakley and Boudreau, 2000), with time and development of understanding of natural patterns in species abundances and distributions, a greater understanding of the Glacier Bay ecosystem will result.

The findings from long-term monitoring may contribute substantially to the ability to assess effects to the intertidal from issues or stressors of concern to the park. Some of these are: oil spills, immigration of sea otters, shifts in species abundances and distributions due to local or global climate change, and invasive species. One potential invasive species that could have large effects on the intertidal communities of Glacier Bay is the green crab, Carcinus maenas. The green crab is spreading north up the west coast of North America, and by 1999 had reached British Columbia (U.S. Environmental Protection Agency, 2009).

Linking intertidal monitoring at Glacier Bay with monitoring planned for Sitka National Historical Park (NHP) would allow more robust assessment of biogeographical changes in species abundances and distributions caused by climate change or the advance of invasive species. Expanding the linkage of Sitka NHP and Glacier Bay NP\&P studies to monitoring programs being set up in Prince William Sound and the NPS Southwest Area Network parks would expand our ability to understand community dynamics across the Gulf of Alaska, and detect effects from broader-scale environmental drivers, including climate change.

One of the major threats of climate change is acidification of the oceans resulting from increased atmospheric $\mathrm{CO}_{2}$ (for example, Orr and others, 2005). Sessile invertebrates with calcareous shells (for example, mussels and barnacles) or whose larval forms have such shells will be particularly vulnerable to changes in ocean acidity. Thus, consistent sampling of ocean water as well as intertidal sessile species may indicate important changes in the marine environment that are not detected so readily in pelagic systems. The visibility and accessibility of marine intertidal communities, as well as their complexity, are some of the reasons why these communities have been studied intensively. This knowledge base increases the potential for understanding changes that may occur, even though it does not eliminate the need for focused research.

Thus, having and implementing an effective monitoring protocol for intertidal communities in Glacier Bay may aid detection and interpretation of major changes that are likely to occur. This study provides the first step in that direction by providing an effective protocol for assessing changes occurring to the major sessile taxa in the rocky intertidal communities. 


\section{Conclusions}

The probability-based survey and sampling approaches used in this study have provided a broad characterization of intertidal habitats within Glacier Bay, as well as statistical comparison of different sampling approaches that varied in their inference, intensity, and techniques. The three levels of surveys included: aerial surveys, CG sampling, and FG sampling. At each level, there were some reductions in inference based on: segments that were classified inappropriately (aerial surveys), segments that could not be sampled (CG sampling), or geographical and habitat restrictions (FG sampling).

Rocky habitat, including cobble/boulder and bedrock substrates, was the focus of on-site surveys. Analyses presented focus on the three predominant sessile taxa: barnacles; the mussel Mytilus trossulus; and the rockweed Fucus distichus subsp. evanescens. The CG sampling, which involved sampling 25 randomly selected sites at relatively low intensity, provided a higher degree of power to detect changes in the predominant sessile taxa than did the more intensive FG sampling of 6 sites. The FG sites, a subset of the CG sites, were cobble/boulder dominated and were located in the lower half of the bay.

I provide data illustrating the effect on power of different sampling methods (quadrats, vertical transects, and horizontal transects), sampling parameters (number of sites, number of transects, intensity of point sampling per meter), and management-set parameters (the level of change to be detected, certainty [alpha]).

Point-intercept sampling of vertical transects had greater power to detect change than did similar sampling of horizontal transects and quadrats. The number of sites sampled had the largest effect on power, followed by the number of transects sampled and alpha levels. The number of points sampled per meter had the least effect on power. There were consistent differences among species in the ability of the sampling to detect changes in their abundances. There was greater power to detect changes in abundance of barnacles, somewhat less power for the mussel Mytilus, and least power for the rockweed Fucus.

The CG sampling regime carried out from 1998 through 2001 would provide the park a means to assess changes to these intertidal taxa and communities on a broad spatial scale. Under this approach, sampling would occur annually (or at greater time intervals) at the 25 established sites, using 6 vertical transects per site, sampled at 5 points $/ \mathrm{m}$. This sampling provides a power of 0.8 or greater, with an alpha of 0.05 , to detect annual changes of 10 percent for barnacles and Mytilus. The ability to determine change for Fucus is somewhat less, particularly for increases in abundance; however, acquiring more data over time should increase the ability to distinguish trends. Further effort is needed to develop effective protocols for small and large mobile invertebrates.

The data from this project provide managers with substantial information that will be useful in designing and implementing a long-term intertidal monitoring program with sufficient power to detect trends for important sessile taxa inhabiting rocky shores within Glacier Bay. Data from this study and future monitoring also may be used to assess impacts to these biological communities from such causes as climate change, invasive species, oil spills, trampling, or the immigration of sea otters. 


\section{Acknowledgments}

This project has been inspired and supported by the input of various statisticians, including Lyman McDonald (WEST, Inc.), Mark Udevitz (USGS), and Alice Shelly (TerraStat). Numerous people have made significant contributions including: Jim Bodkin, Kim Kloecker, Tammy Gage, Megan Ferguson, Karen Vandersall, George Esslinger, Dan Monson, Jennifer Mondragon, Jeffrey Mondragon, Amy Delorenzo, Todd Stoltey, Erica Madison, Jim de la Bruere, Dave Douglas (USGS); Mandy Lindeberg (National Oceanic and Atmospheric Administration); Jennifer Williams (Sitka NHP); and Lewis Sharman, Bill Eichenlaub, Mary Beth Moss, and Larry Basch (Glacier Bay NP\&P). In addition to NPS staff input, I appreciate the financial support for the fourth year of sampling and logistical support. Special thanks to Bill Eichenlaub, Jennifer Mondragon, and Jeffrey Mondragon for creation of the MicrosoftC Access database. Additional thanks to reviewers of different drafts of this report (Karen Oakley, Eric Knudsen, Heather Coletti, Erik Beever, Lyman McDonald, and Tony DeGange). Initial funding was from USGS National Resources Preservation Program; additional support was provided by the National Park Service (Glacier Bay NP\&P), and the U.S. Geological Survey. 


\section{References Cited}

Abacus Concepts, Inc., 1996, StatView for Windows, version 4.57: Berkeley, CA, Abacus Concepts, Inc.

Ambrose, R.F., Engle, J.M., Raimondi, P.T., Wilson, M., and Alstatt, J.A., 1995, Rocky intertidal and subtidal resources: Santa Barbara County mainland: Report to the Minerals Management Service, Pacific OCS Region, OCS Study MMS 95-0067.

Bodkin, J.L., Ballachey, B.B., Esslinger, G.G., Kloecker, K.A., Monson, D. H., and Coletti, H.A., 2007, Perspectives on an invading predator-Sea otters in Glacier Bay, in Piatt, J.F., and Gende, S.M., eds., Proceedings of the Fourth Glacier Bay Science Symposium, October 2628, 2004: U.S. Geological Survey Scientific Investigations Report 2007-5047, p. 133-136.

Bodkin, J.L., Dean, T.A., Coletti, H.A., and Kloecker, K.A., 2008, Nearshore marine vital signs monitoring in the Southwest Alaska network of National Parks: Annual Report to the National Park Service, Anchorage, AK, $176 \mathrm{p}$.

Connell, J.H., 1961, The influence of interspecific competition and other factors on the distribution of the barnacle Chthamalus stellatus: Ecology, v. 42, p. 710-723.

Cubit, J.D., and Connor, J.L., 1993, Effects of the 1986 Bahía las Minas oil spill on reef flat communities, in Proceedings of the 1993 International Oil Spill Conference: Washington, D.C., American Petroleum Institute, p. 329-334.

Dayton, P.K., 1971, Competition, disturbance, and community organization: The provision and subsequent utilization of space in a rocky intertidal community: Ecological Monographs, v. 41, p. 351-389.

Drew, G.S., Speckman, S.G., Piatt, J.F., Burgos, J.M., and Bodkin, J.L., 2008, Survey design considerations for monitoring marine predator populations in Glacier Bay, Alaska: Results and post-hoc analysis of surveys conducted in 1999-2003: Anchorage, AK, U.S. Geological Survey Administrative Report, $127 \mathrm{p}$.

Geiselman, J., Dunlap, J., Hooge, P., and Albert, D., 1997, Glacier Bay ecosystem Geographic Information System, CD-ROM: U.S. Geological Survey-Interrain Pacific, Anchorage, AK, and Portland, OR.

Gibbs, J.P., 1995, MONITOR, Software for estimating power of population monitoring programs to detect trends in plant and animal abundance (versions 6.2, modified exponential, and 10.0). (Version 6.2 user manual available at http://www.wcsmalaysia.org/analysis/PDF/MONITORmanual.pdf.)

Gibbs, J.P., and Ramirez de Arellano, P., 2007, Program MONITOR: Estimating the statistical power of ecological monitoring programs: Version 10.0.0. URL: www.esf.edu/efb/gibbs/monitor/monitor.htm.

Hatch, S.A., 2003, Statistical power for detecting trends with applications to seabird monitoring: Biological Conservation, v. 111, no. 3, p. 317-329.

Highsmith, R.C., Rucker, T.L., Stekoll, M.S., Saupe, S.M., Lindeberg, M.R., Jenne, R.N., and Erickson, W.P., 1996, Impact of the Exxon Valdez oil spill on intertidal biota, in Rice, S.D., Spies, R.B., Wolfe, D.A., and Wright, B.A., eds., Proceedings of the Exxon Valdez Oil Spill Symposium: American Fisheries Society Symposium Number 18: Bethesda, MD, American Fisheries Society, p. 212-237. 
Irvine, G.V., 1998, Development of coastal monitoring protocols and process-based studies to address landscape-scale variation in coastal communities of Glacier Bay National Park and Preserve, Katmai National Park and Preserve, and Wrangell-St. Elias National Park and Preserve; Phase II: Development and testing of monitoring protocols for selected intertidal habitats and assemblages: National Resources Preservation Program Project Annual Report, March 1998, $62 \mathrm{p}$.

Irvine, G.V., 2000, Persistence of spilled oil on shores and its effects on biota, in Sheppard, Charles, ed., Seas at the millennium: An environmental evaluation, Volume III, Global issues and processes: Elsevier Science, Oxford, p. 267-281.

Irvine, G.V., and Madison, E.N., 2008, Development of a monitoring protocol to detect ecological change in the intertidal zone of Sitka National Historical Park, Alaska: U.S. Geological Survey Scientific Investigations Report 2008-5139, 58 p. (Also available at http://pubs.usgs.gov/sir/2008/5139.)

Irvine, G., Stevens, D., Deschu, N., Dickison, G., Rice, B., and Somers, G., 1994, Coastal resources in Alaska: What's the program: Report to the Superintendent's Conference, Alaska Regional Office, National Park Service, November 1994.

Kinnetic Laboratories, Inc., 1992, Study of the rocky intertidal communities of central and northern California (final report): Vol. I: Prepared in association with the University of California, Santa Cruz, Moss Landing Marine Laboratories, and TENERA Corporation for the Pacific OCS Region, Minerals Management Service, U.S. Department of the Interior, contract no. 14-12-0001-30057, OCS Study, MMS 91-0089, 321 p.

McDonald, L.L., Erickson, W.P., and Strickland, M.D., 1995, Survey design, statistical analysis, and basis for statistical inferences in coastal habitat injury assessment: Exxon Valdez oil spill, in Wells, P.G., Butler, J.N., and Hughes, J.S., eds., Exxon Valdez oil spill: Fate and effects in Alaskan waters: ASTM STP 1219, p. 296-311.

McDonald, T., 2004, GRTS for the average Joe: A GRTS sampler for Windows: West, Inc., 10 p. (Also available http://www.west-inc.com/reports/grts.pdf.)

Miller, A.W., and Ambrose, R.F., 2000, Sampling patchy distributions: comparison of sampling designs in rocky intertidal habitats: Marine Ecology Progress Series 196, p. 1-14.

Minchinton, T.E., and Raimondi, P.T., 2001, Long-term monitoring of rocky intertidal communities at the Channel Islands National Park: Summary of spatial and temporal trends and statistical power analyses: Report for the Scientific and Management Review of Monitoring Protocols for the Channel Islands National Park, 29 p.

Murray, S.N., Ambrose, R.N., and Dethier, M.N., 2006, Monitoring Rocky Shores: University of California Press, Berkeley, 220 p.

Nautical Software, Inc., 1997, Tides \& Currents for Windows, version 2.5a. (Note: Nautical Software was acquired by Nobeltec in 1999; newer version of program available at http://cms.nobeltec.com/cms/Products/NavigationData/TidesCurrents.aspx)

Oakley, K.L., and Boudreau, S.L., 2000, Conceptual design of the long-term ecological monitoring program for Denali National Park and Preserve, $116 \mathrm{p}$.

Orr, J.C., and others, 2005, Anthropogenic ocean acidification over the twenty-first century and its impact on calcifying organisms: Nature, v. 437 (September 29, 2005), doi: 10.1038/ nature04095, p. 681-686.

Osenberg, C.W., Schmitt, R.J., Holbrook, S.J., Abu-Saba, K., and Flegal, A.R., 1996, Detection of environmental impacts: natural variability, effect size, and power analysis, in Schmitt, R.J., and Osenberg, C.W., eds., Detecting ecological impacts: concepts and applications in coastal habitats: Academic Press, San Diego, chap. 6, p. 83-107. 
Paine, R.T., 1966, Food web complexity and species diversity: The American Naturalist, v. 100, no.910, p. 65-75.

Peterson, C.H., McDonald, L.L., Green, R.H., and Erickson, W.P., 2001, Sampling design begets conclusions: the statistical basis for detection of injury to and recovery of shoreline communities after the Exxon Valdez oil spill: Marine Ecology Progress Series, v. 210, p. 255283.

Raimondi, P.T., Ambrose, R.F., Engle, J.M., Murray, S.N., and Wilson, M., 1999, Monitoring of rocky intertidal resources along the central and southern California mainland: 3-year report for San Luis Obispo, Santa Barbara, and Orange Counties (fall 1995-spring 1998): U.S. Department of the Interior, Minerals Management Service MMS 99-0032, Pacific OCS Region, $143 \mathrm{p}$.

Richards, D.V., and Davis, G.E., 1988, Rocky intertidal communities monitoring handbook, Channel Islands National Park, California, National Park Service, Ventura, CA, 78 p.

Schmitt, R.J., and Osenberg, C.W., eds., 1996, Detecting ecological impacts: Concepts and applications in coastal habitats: Academic Press, San Diego, 401 p.

Schroeter, S.C., Dixon, J.D., Kastendiek, J., Smith, R.O., and Bence, J.R., 1993, Detecting the ecological effects of environmental impacts: A case study of kelp forest invertebrates: Ecological Applications, v. 3, no, 2, p. 331-350.

Sharman, Lewis, 1990, Marine intertidal community; development following glacial recession in Glacier Bay, Alaska, in Milner, A.M., and Wood, J.D., Jr., eds., Proceedings of the Second Glacier Bay Science Symposium, September 19-22, 1988, p. 108-115.

Sousa, W.P., 1979, Disturbance in marine intertidal boulder fields: The nonequilibrium maintenance of species diversity: Ecology, v. 60, p. 1125-1239.

Stevens, D.L., Jr., and Olsen, A.R., 2004, Spatially balanced sampling of natural resources: Journal of the American Statistical Association, v. 99, no. 465, p. 262-278. (Also available at http://www.epa.gov/nheerl/arm/documents/grts_asa.pdf.)

Stewart-Oaten, A., Murdoch, W.W., and Parker, K.R., 1986, Environmental impact assessment: Pseudo-replication in time?: Ecology, v. 67, p. 929-940.

Thomas, L., and Krebs, C.J., 1997, A review of statistical power analysis software: Bulletin of the Ecological Society of America, v. 78, no. 2, p. 126-139.

Underwood, A.J., 1994, On beyond BACI: sampling designs that might reliably detect environmental disturbances: Ecological Applications 4, p. 3-15.

U.S. Environmental Protection Agency, 2009, Non-native crab species: Region 10: Pacific Northwest, accessed July 2009, at http://yosemite.epa.gov/r10/ECOCOMM.NSF/B724CA698F6054798825705700693650/3BD4 70D756FBBFAE88257416005A73DE? OpenDocument .

Zar, J.H., 1984, Biostatistical analysis: Prentice-Hall, Inc., NJ, 718 p. 


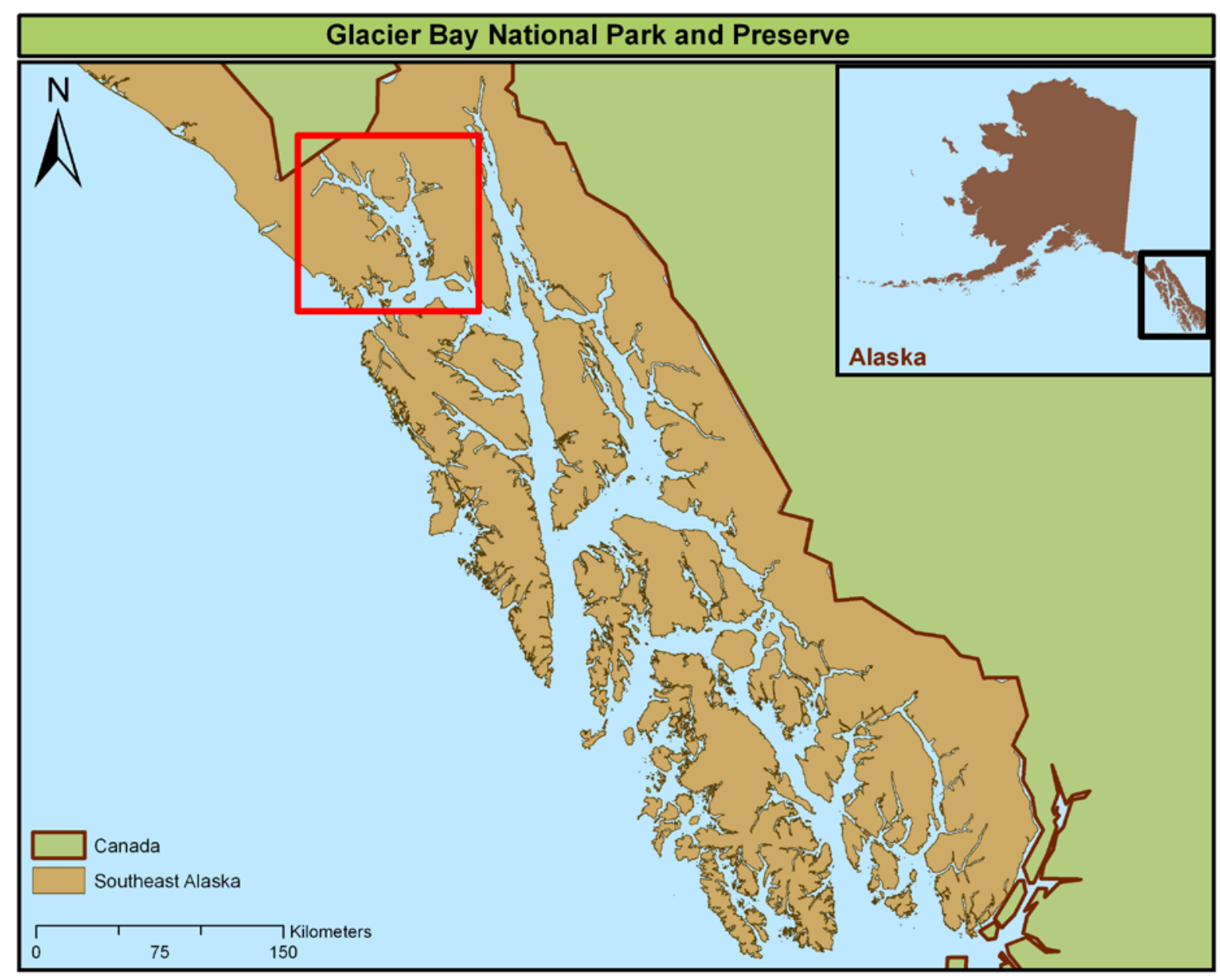

Figure 1. Location of Glacier Bay National Park and Preserve (red box) in southeast Alaska (black box in inset). 


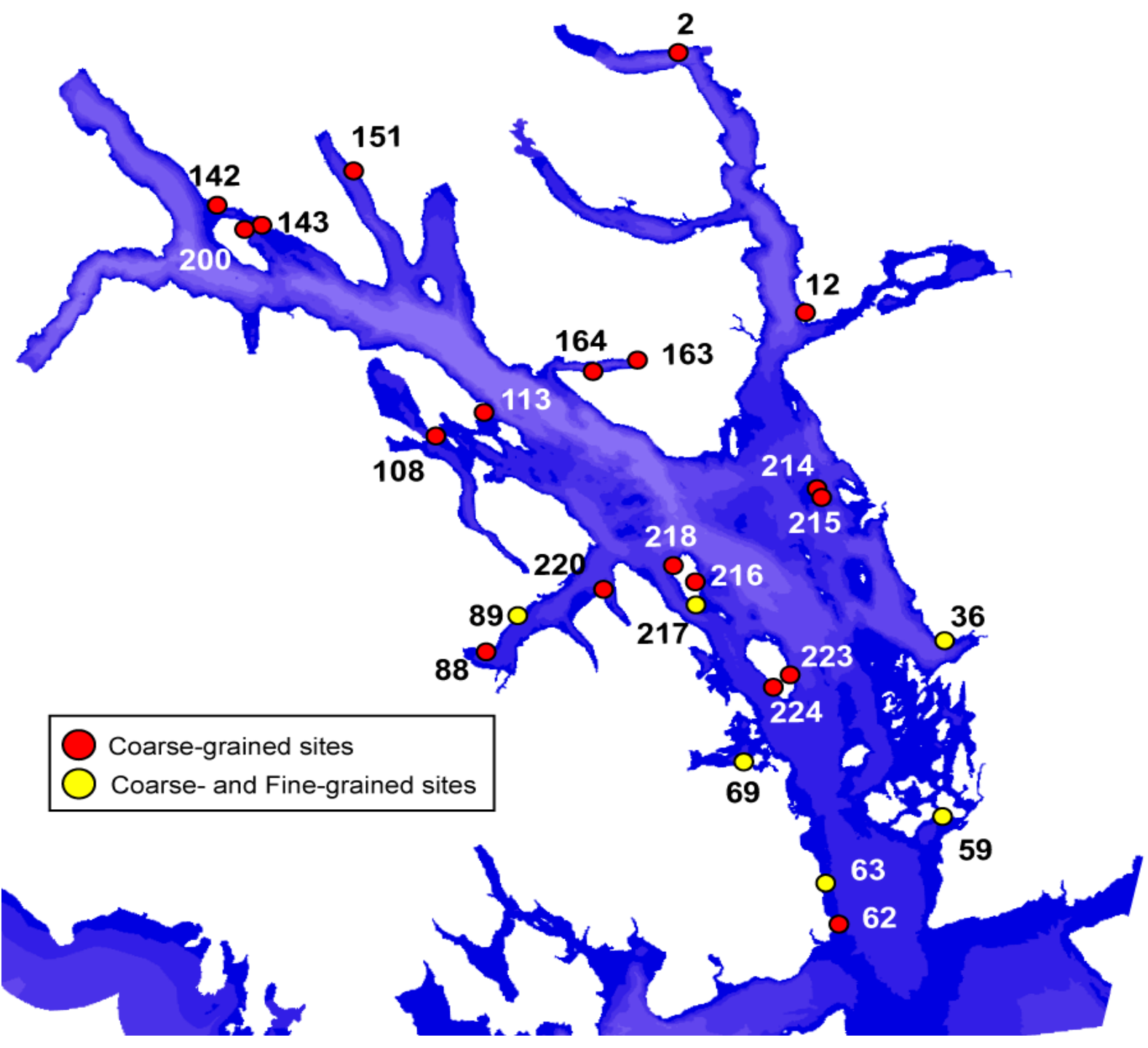

Figure 2. Locations and segment numbers of the coarse-grained and fine-grained sites in Glacier Bay National Park and Preserve. Fine-grained sites are a subset of the coarse-grained sites. 


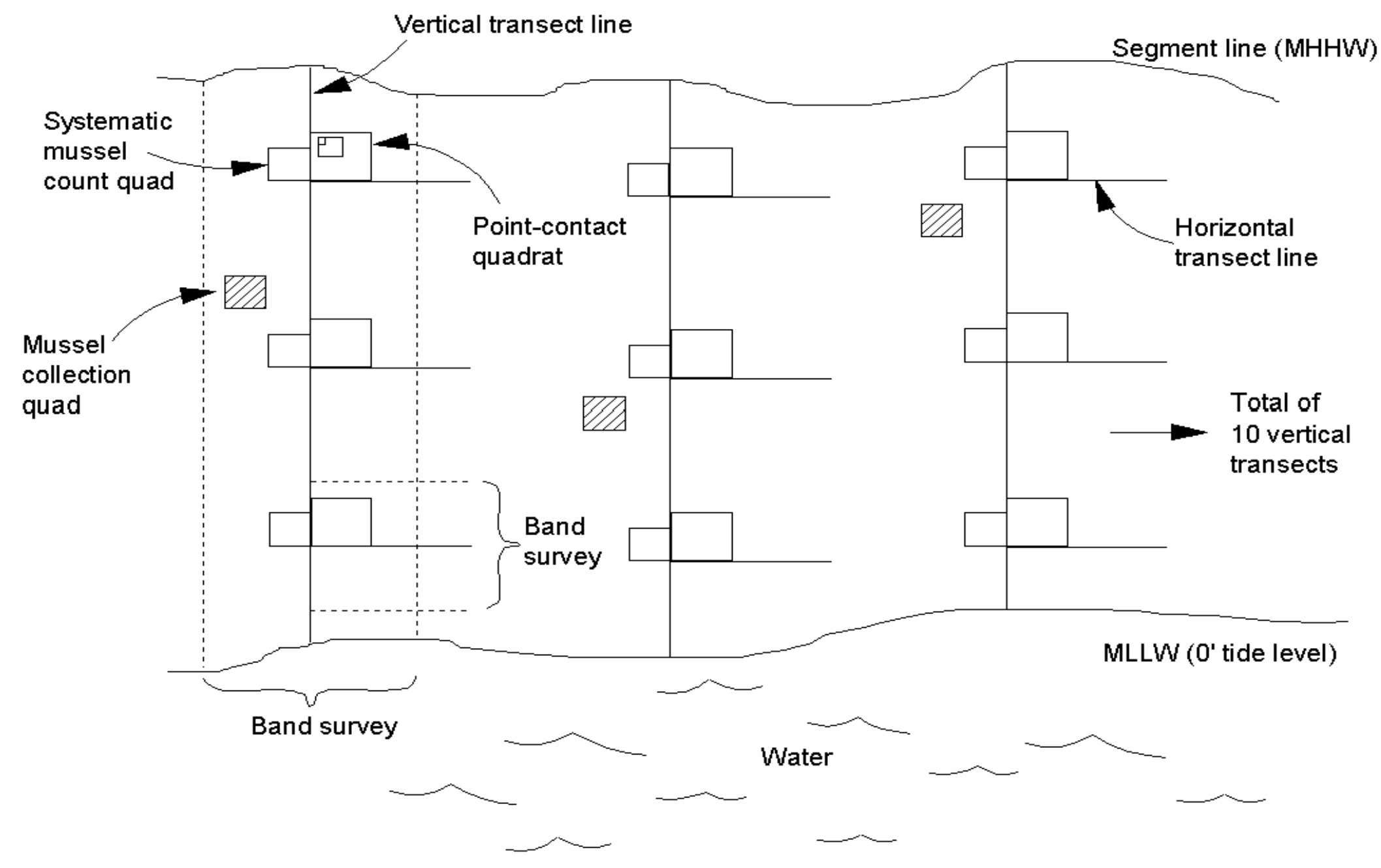

Figure 3. Layout of the various sampling methods used during the fine-grained surveys. 


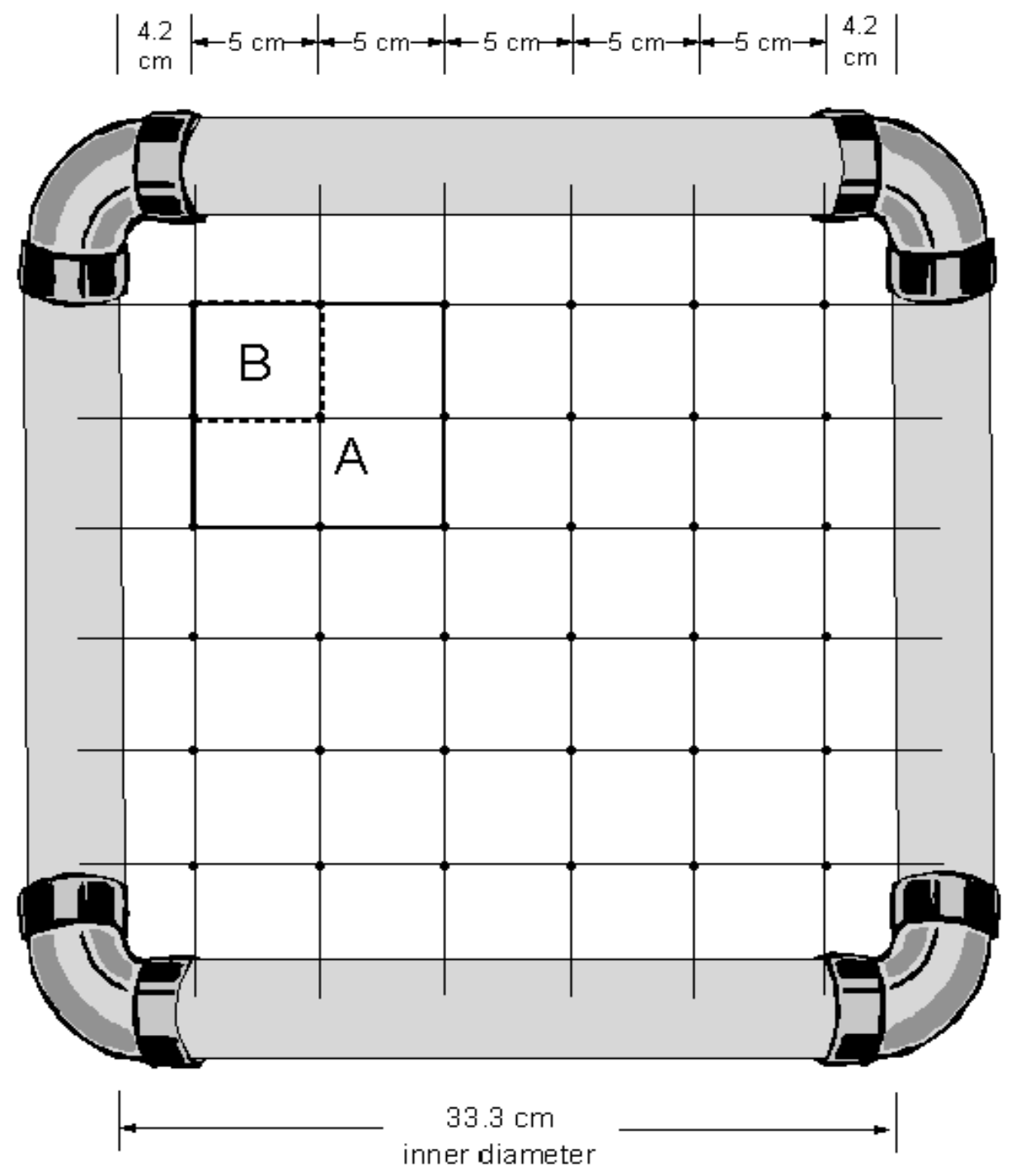

\section{A - $10 \times 10 \mathrm{~cm}$ - Littorine sampling area \\ B - $5 \times 5 \mathrm{~cm}$ - Barnacle spat sampling area}

Figure 4. Quadrat used for point-contact sampling at the fine-grained sampling level. Littorine snails and barnacle spat/recruits were subsampled in the indicated areas. 

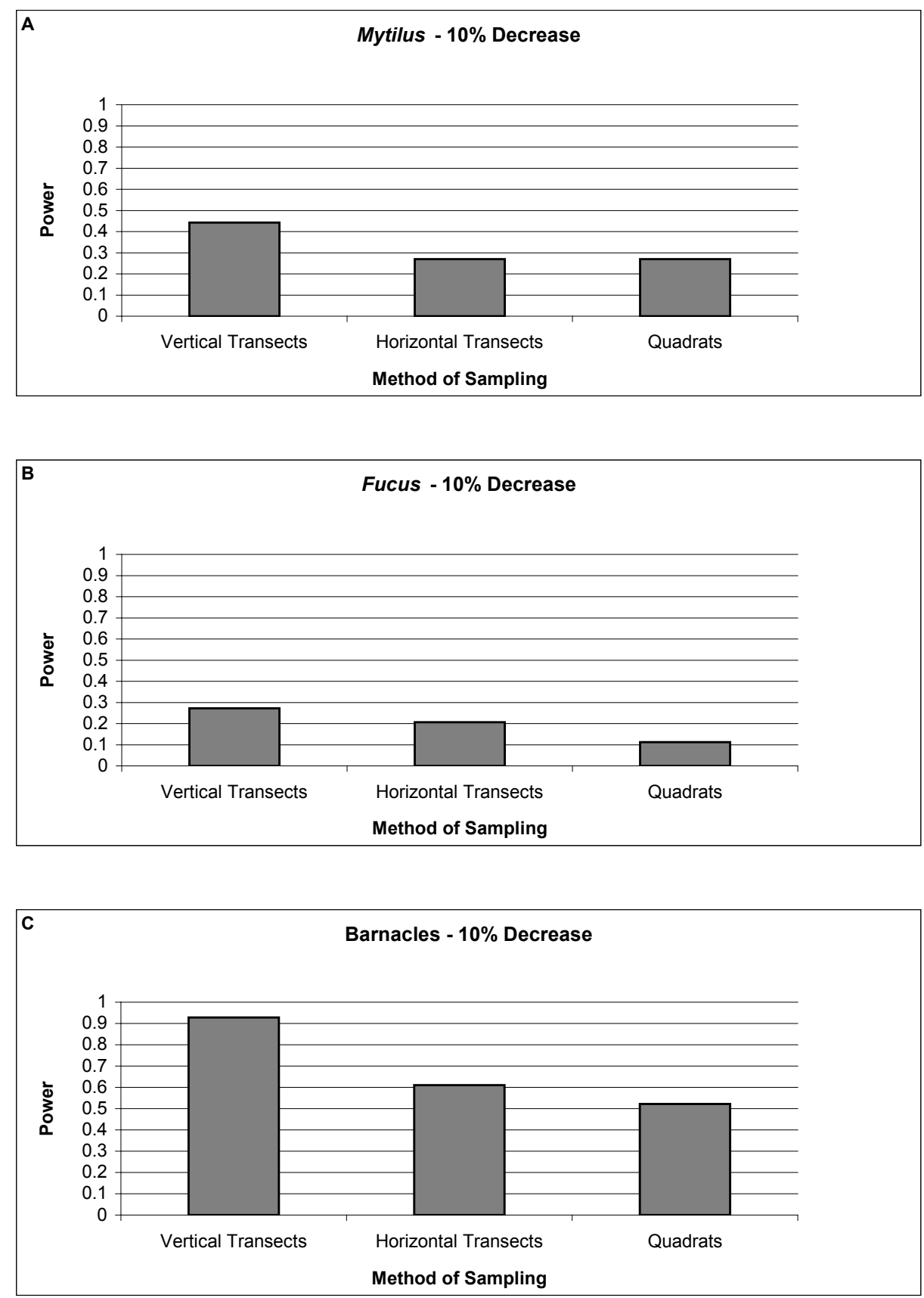

Figure 5. Comparison of the power of three point-intercept sampling methods conducted as part of the finegrained sampling protocol in 1997. At six sites, 10 vertical transects, 30 horizontal transects, and 30 quadrats were sampled. A. Power to detect a 10 percent decrease in Mytilus. B. Power to detect a 10 percent decrease in Fucus. C. Power to detect a 10 percent decrease in barnacles. $\alpha=0.05$. 


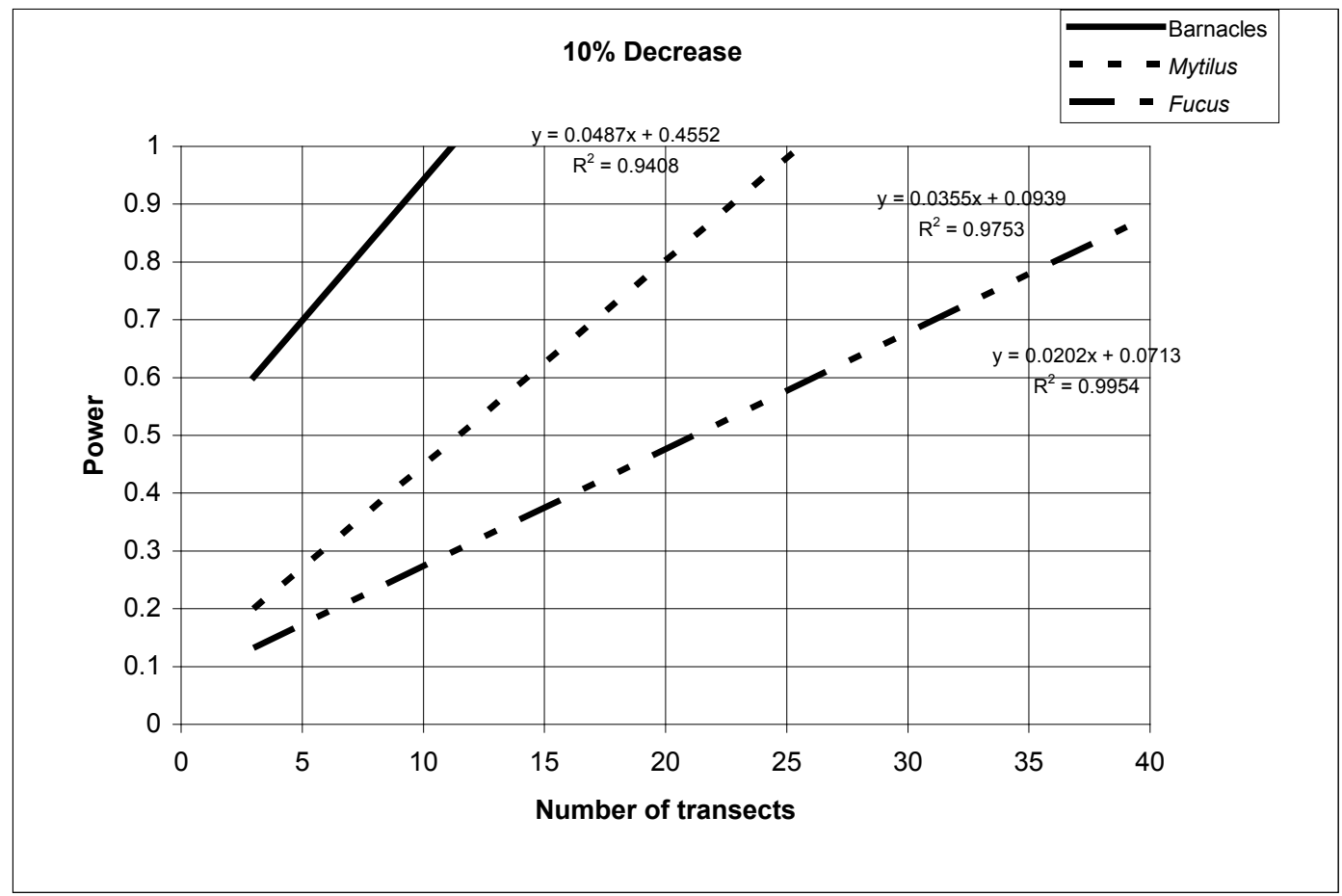

Figure 6. Power to detect a 10 percent decrease in the predominant sessile species as related to the number of transects sampled. A linear trend is projected based on data collected from 1997 fine-grained sampling of six sites. Each transect was sampled at a frequency of 5 points $/$ meter. $\alpha=0.05$. 


\section{Barnacles}

$\begin{array}{cc}\text { Population Trend } & \text { Power } \\ -10 \% & 1 \\ -5 \% & 0.904 \\ -3 \% & 0.458 \\ -2 \% & 0.28 \\ -1 \% & 0.092 \\ 0 \% & 0.068 \\ 1 \% & 0.094 \\ 2 \% & 0.212 \\ 3 \% & 0.446 \\ 5 \% & 0.862 \\ 10 \% & 1\end{array}$

\section{Mytilus}

$\begin{array}{cc}\text { Population Trend } & \text { Power } \\ -10 \% & 1 \\ -5 \% & 0.83 \\ -3 \% & 0.424 \\ -2 \% & 0.23 \\ -1 \% & 0.072 \\ 0 \% & 0.04 \\ 1 \% & 0.09 \\ 2 \% & 0.222 \\ 3 \% & 0.42 \\ 5 \% & 0.768 \\ 10 \% & 1\end{array}$

\section{Fucus}

$\begin{array}{cc}\text { Population Trend } & \text { Power } \\ -10 \% & 0.974 \\ -5 \% & 0.49 \\ -3 \% & 0.228 \\ -2 \% & 0.124 \\ -1 \% & 0.072 \\ 0 \% & 0.052 \\ 1 \% & 0.052 \\ 2 \% & 0.132 \\ 3 \% & 0.188 \\ 5 \% & 0.422 \\ 10 \% & 0.944\end{array}$
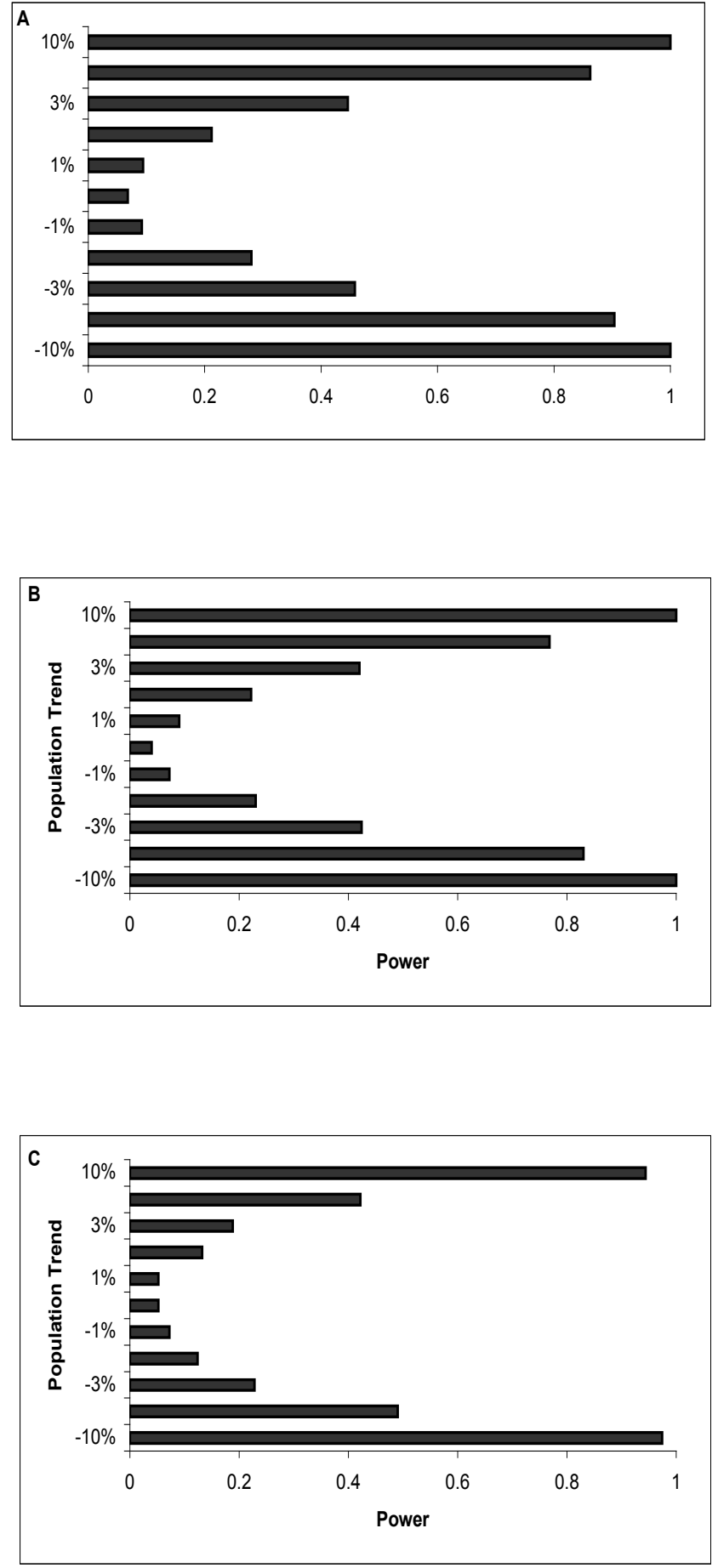

Figure 7. Power to detect population trends based on 4 years of data (1997-2001) in $A$. barnacles; $B$. Mytilus; and C. Fucus. Data are based on sampling of 25 sites, 6 transects, 1 point $/$ meter. $\alpha=0.05$. 


\section{Barnacles}

Population Trend

$-10 \%$

Power

$-5 \%$

0.998

$-3 \%$

0.74

$-2 \%$

0.398

$-1 \%$

0.158

$-0 \%$

0.088

$1 \%$

0.056

$2 \%$

0.088

$3 \%$

0.148

$5 \%$

0.308

$10 \%$

0.74

0.996

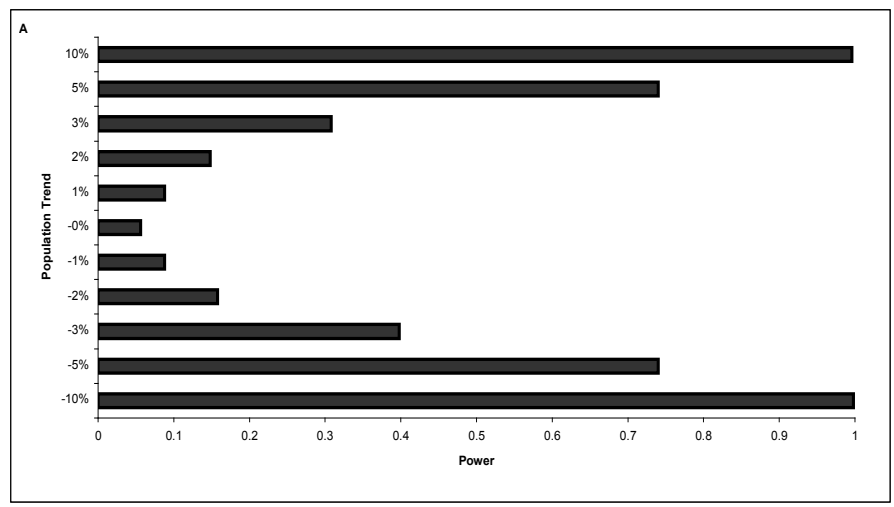

\section{Mytilus}

Population Trend

$\begin{array}{cc}-10 \% & 1 \\ -5 \% & 0.838 \\ -3 \% & 0.456 \\ -2 \% & 0.17 \\ -1 \% & 0.082 \\ -0 \% & 0.066 \\ 1 \% & 0.092 \\ 2 \% & 0.216 \\ 3 \% & 0.43 \\ 5 \% & 0.776 \\ 10 \% & 1\end{array}$

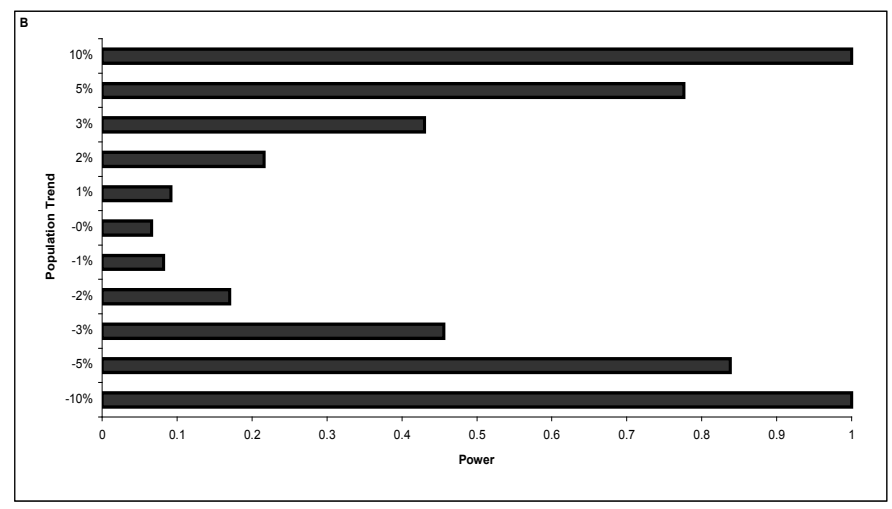

\section{Fucus}

Population Trend

$\begin{array}{cc}-10 \% & 0.912 \\ -5 \% & 0.366 \\ -3 \% & 0.162 \\ -2 \% & 0.092 \\ -1 \% & 0.06 \\ -0 \% & 0.034 \\ 1 \% & 0.062 \\ 2 \% & 0.08 \\ 3 \% & 0.158 \\ 5 \% & 0.37 \\ 10 \% & 0.86\end{array}$

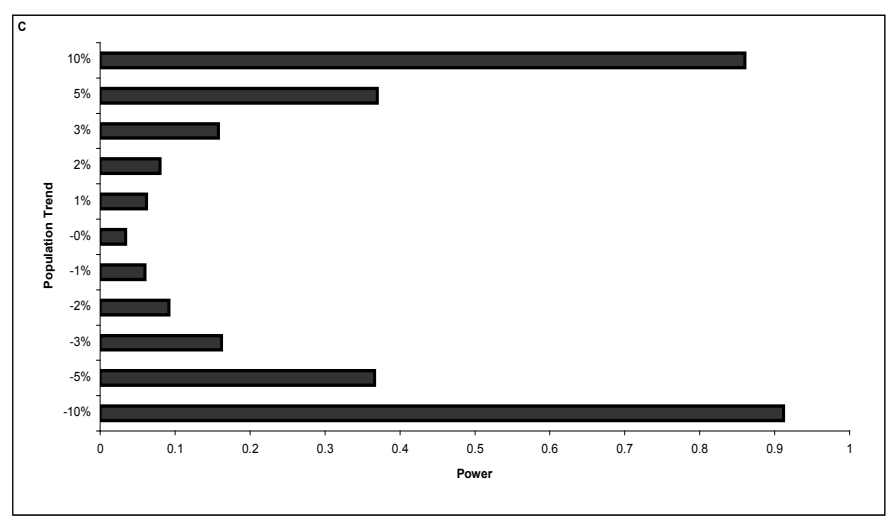

Figure 8. Power to detect population trends based on 3 years of data (1998-2001) in A. barnacles; $B$. Mytilus; and C. Fucus. Sampling was conducted at 25 sites, 6 transects, 5 points/meter. $\alpha=0.05$. 

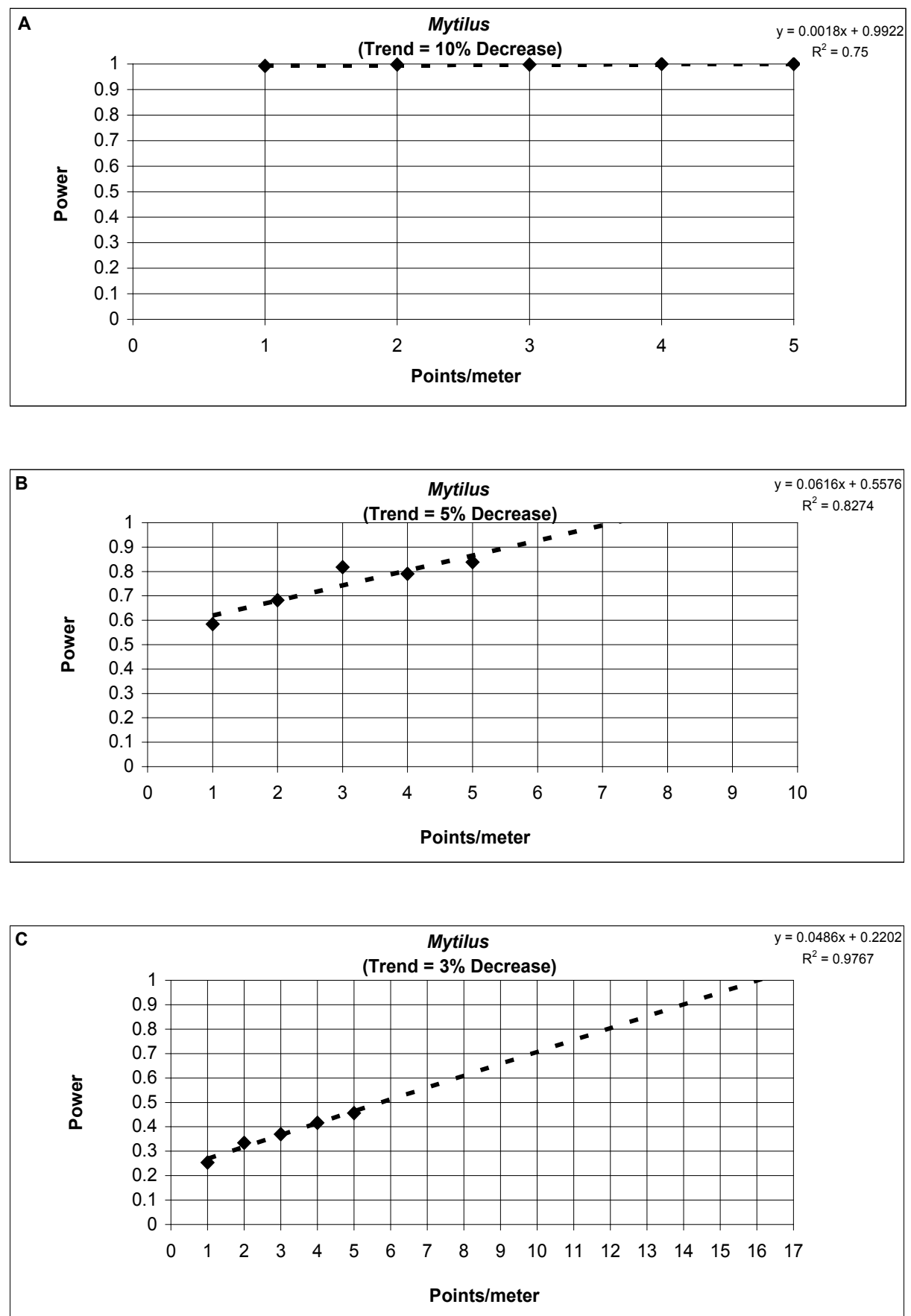

Figure 9. Power to detect decreases in Mytilus populations as related to the number of points sampled per meter of each transect. Data are based on 3 years of sampling (1998-2001) at 25 sites, 6 transects at each site. $\alpha=0.05$. $A .10$ percent decrease; $B .5$ percent decrease; $C .3$ percent decrease. Points on graphs indicate data; lines are linear projections of the trends suggested by these data. 

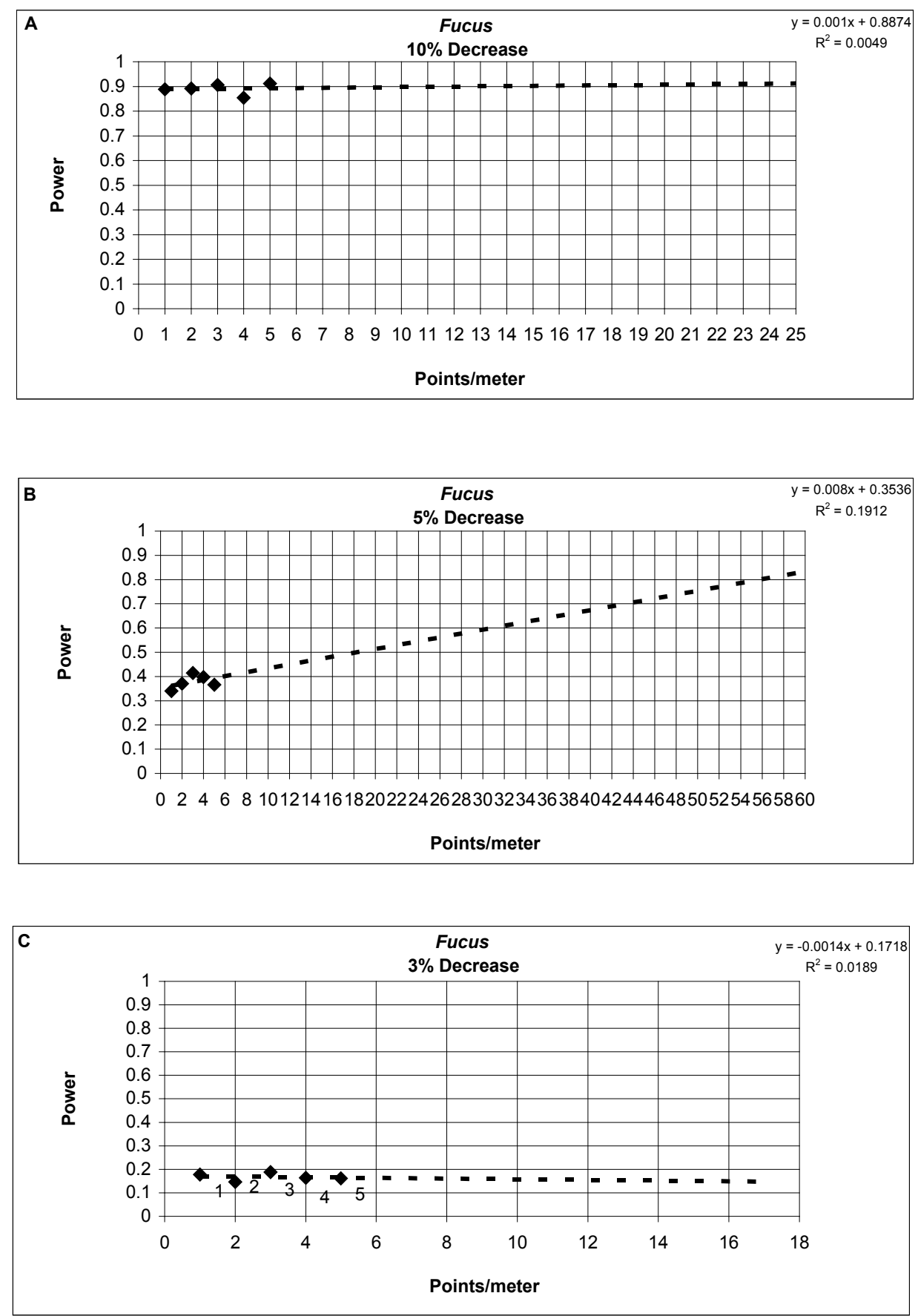

Figure 10. Power to detect decreases in Fucus populations as related to the number of points sampled per meter of each transect. Data are based on 3 years of sampling (1998-2001) at 25 sites, 6 transects at each site. $\alpha=0.05$. $A .10$ percent decrease; $B .5$ percent decrease; $C$. 3 percent decrease. Points on graphs indicate data; lines are linear projections of the trends suggested by these data. 

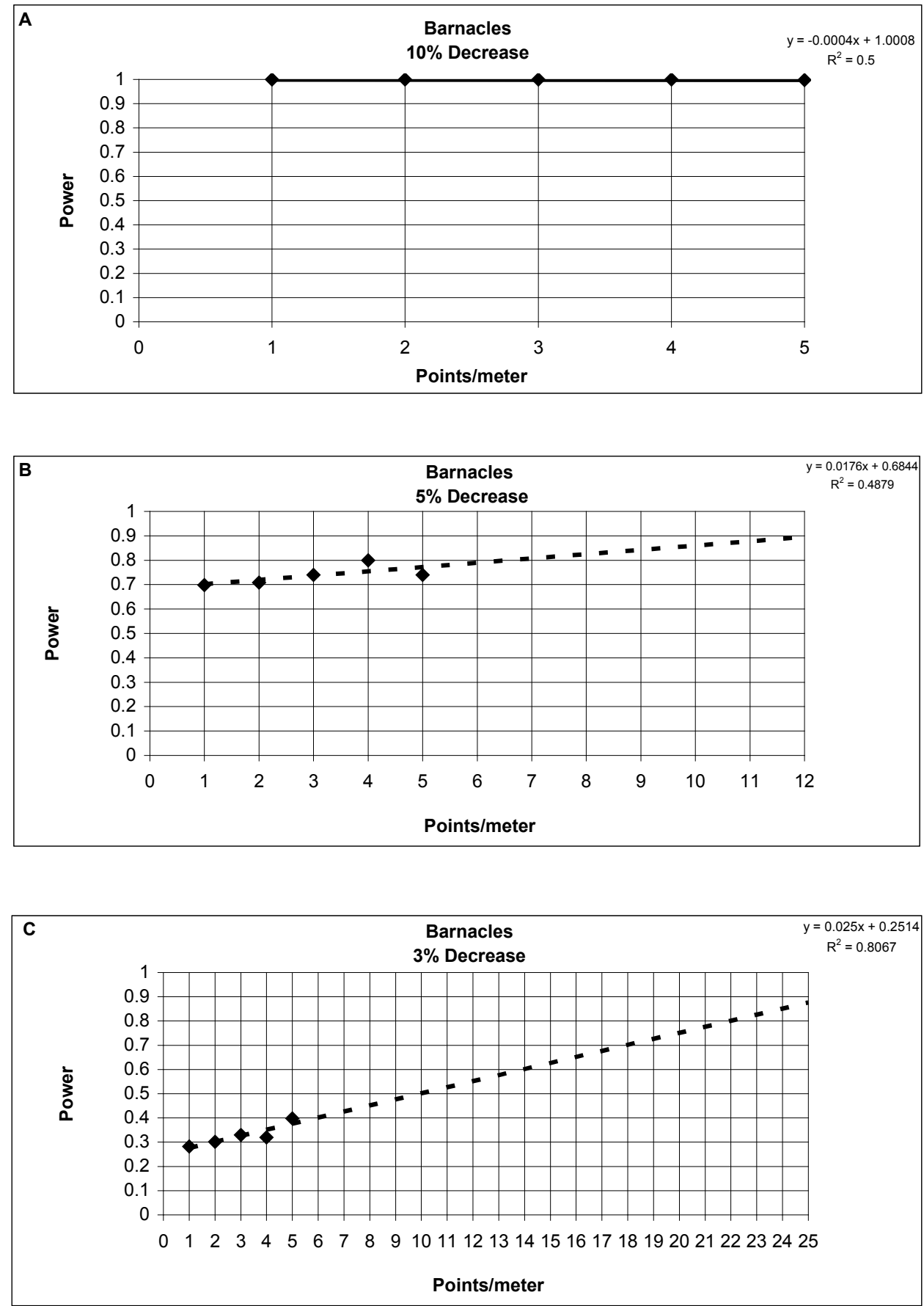

Figure 11. Power to detect decreases in barnacle populations as related to the number of points sampled per meter of each transect. Data are based on 3 years of sampling (1998-2001) at 25 sites, 6 transects at each site. $\alpha=0.05$. $A$. 10 percent decrease; $B .5$ percent decrease; $C$. 3 percent decrease. Points on graphs indicate data; lines are linear projections of the trends suggested by these data. 

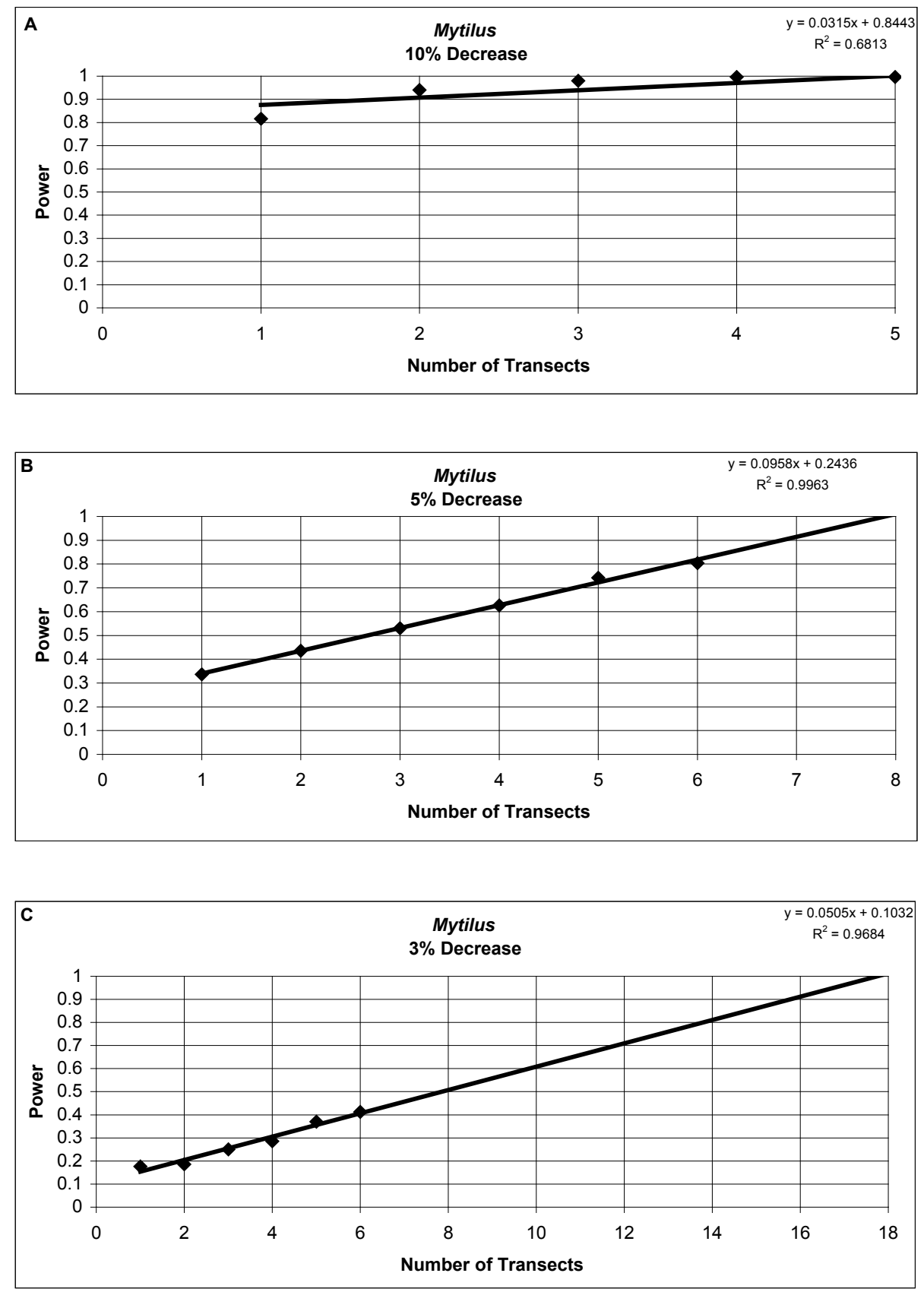

Figure 12. Power to detect a decrease in Mytilus populations as a function of the number of transects sampled at each site. Data are based on 3 years of sampling (1998-2001), 25 sites sampled, transects sampled at a frequency of 5 points/meter. A. 10 percent decrease; $B .5$ percent decrease; $C$. 3 percent decrease, $\alpha=0.05$. Points on graphs indicate data; lines are linear projections of the trends suggested by these data. 

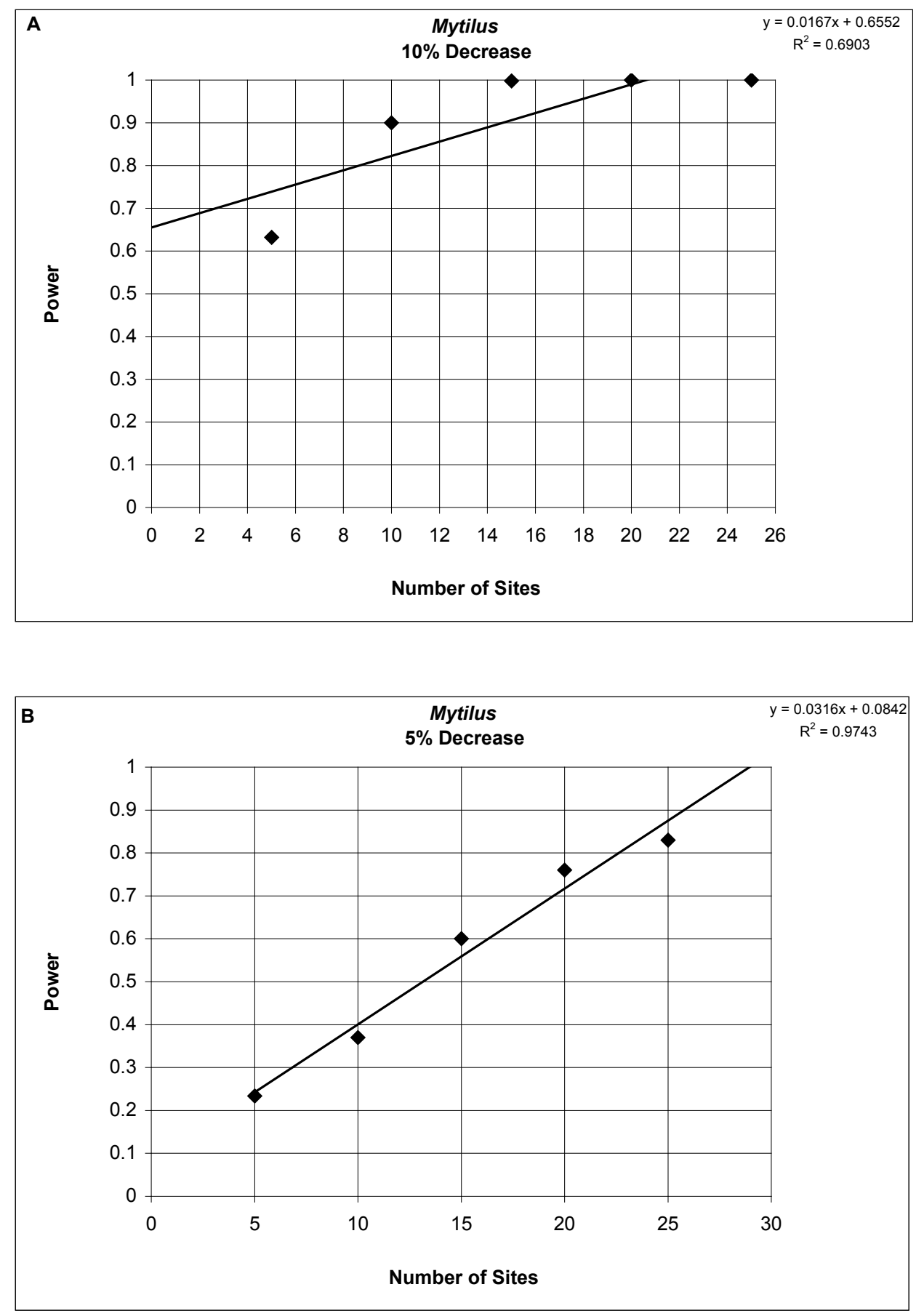

Figure 13. Power to detect a decrease in Mytilus populations in relation to the number of sites. A. 10 percent decrease; B. 5 percent decrease. Data are based on 4 years (1997-2001) sampling of 6 transects sampled at 1 point $/$ meter at each site. $\alpha=0.05$. Points on graphs indicate data; lines are linear regressions of trends suggested by these data. 

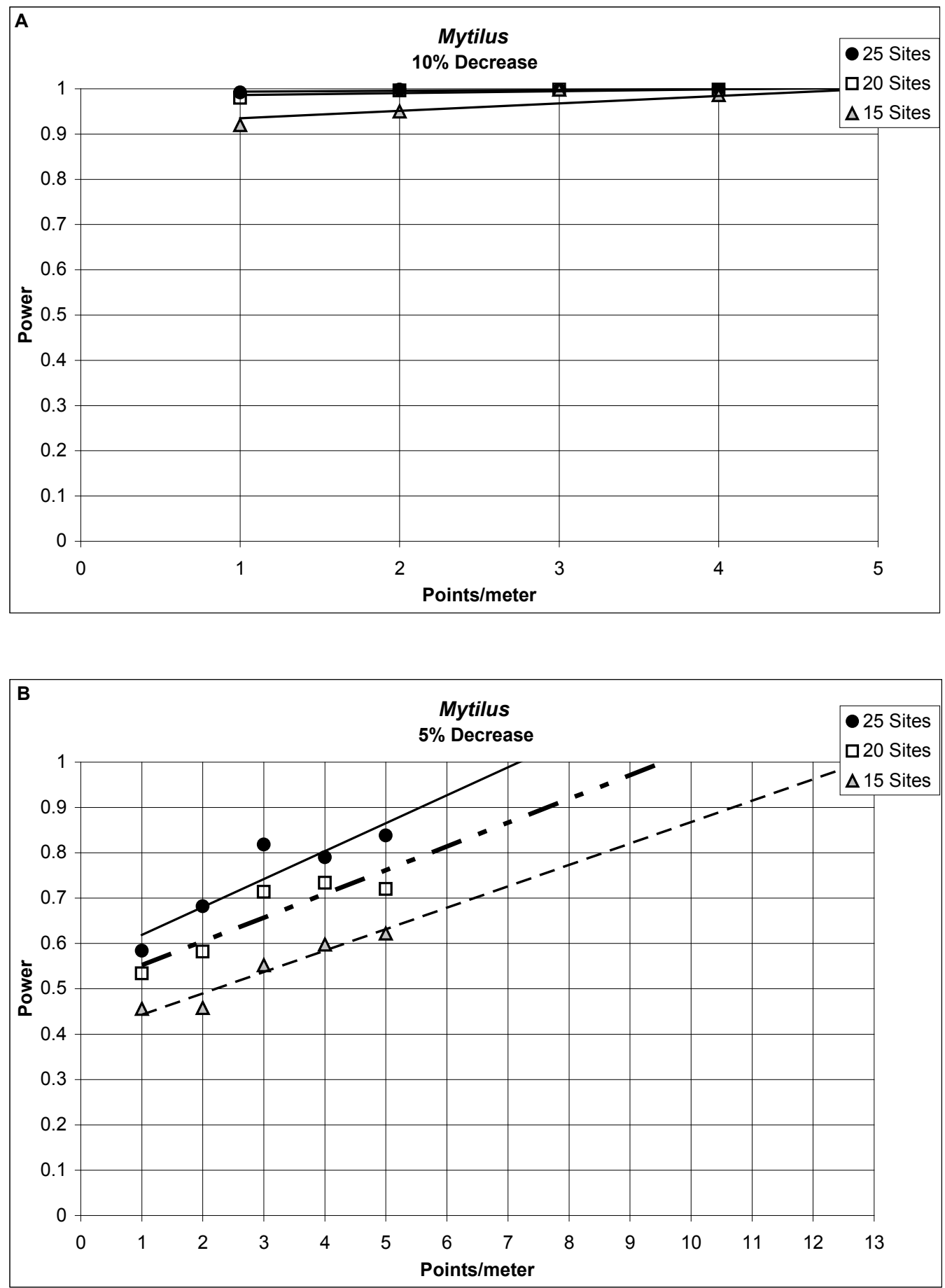

Figure 14. Change in the power to detect a decrease in Mytilus populations as a result of varying the number of points sampled per meter with respect to 25,20 , and 15 sites. $A$. 10 percent decrease; $B .5$ percent decrease. Data are based on 3 years (1998-2001) of sampling 6 transects at each site. $\alpha=0.05$. Points on graphs indicate data; lines are linear regressions of trends suggested by these data. 

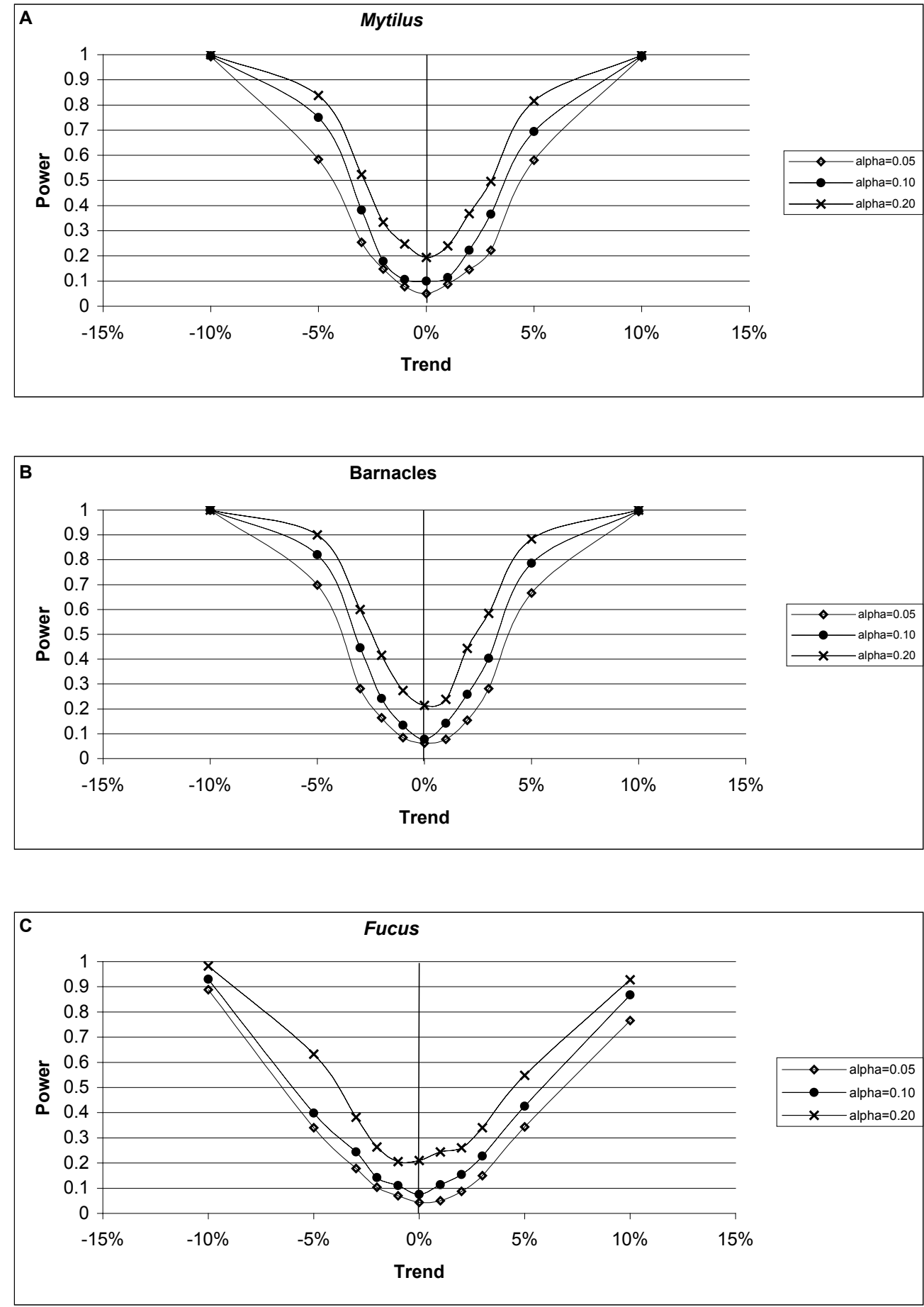

Figure 15. Power to detect trends of differing magnitudes at three levels of alpha $(\alpha=0.05,0.10,0.20)$. Data are based on 4 years (1997-2001) of coarse-grained sampling: 25 sites, 6 transects/site, 1 point/meter. A. Mytilus; B. barnacles; C. Fucus. 


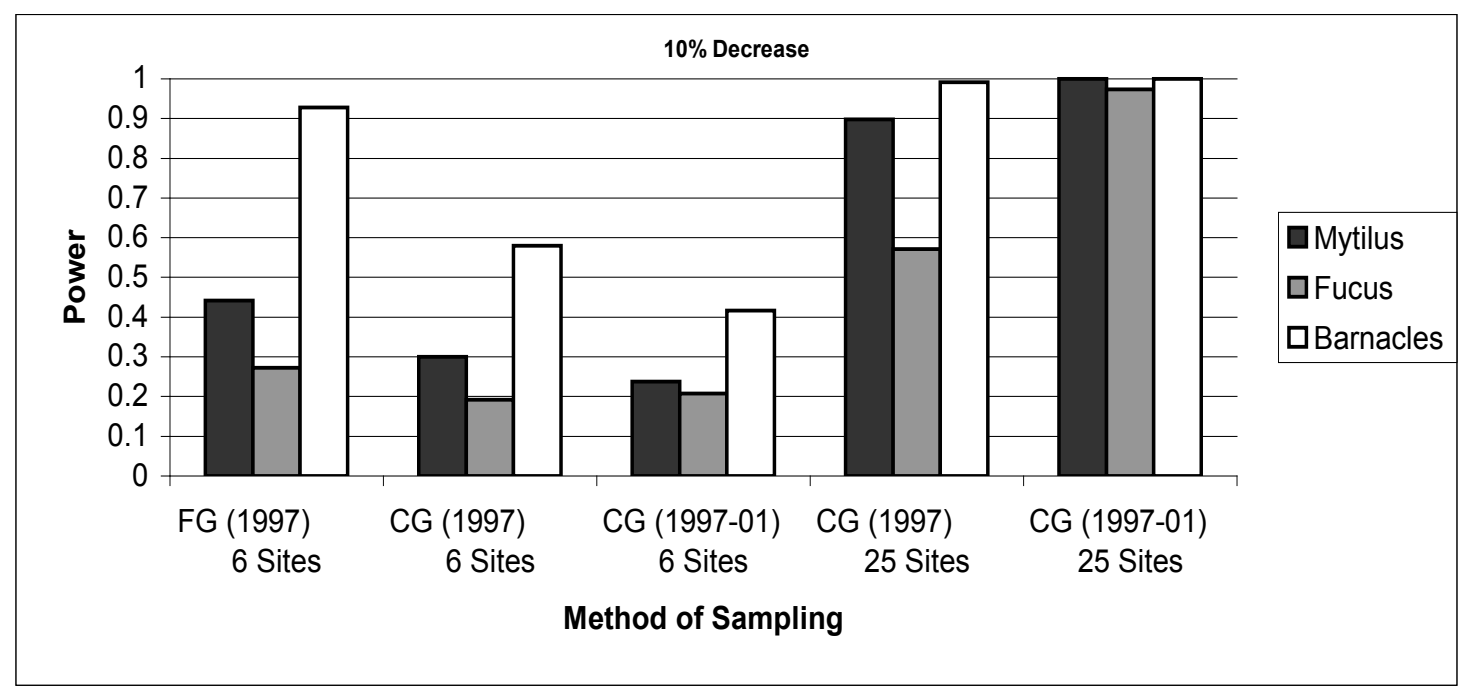

Figure 16. Comparison in the power of fine-grained and coarse-grained vertical transect sampling to detect a 10 percent decrease in Mytilus, Fucus, and barnacles. Fine-grained sampling (1997 only) included 10 transects at each of 6 sites, each transect sampled at 5 points/meter. Coarse-grained data (1997-2001) used in these analyses are derived from sampling 6 transects/ site at 1 point/meter. Analyses of both coarse-grained sampling at the 6 fine-grained sites and all 25 sites are included as a comparison. 

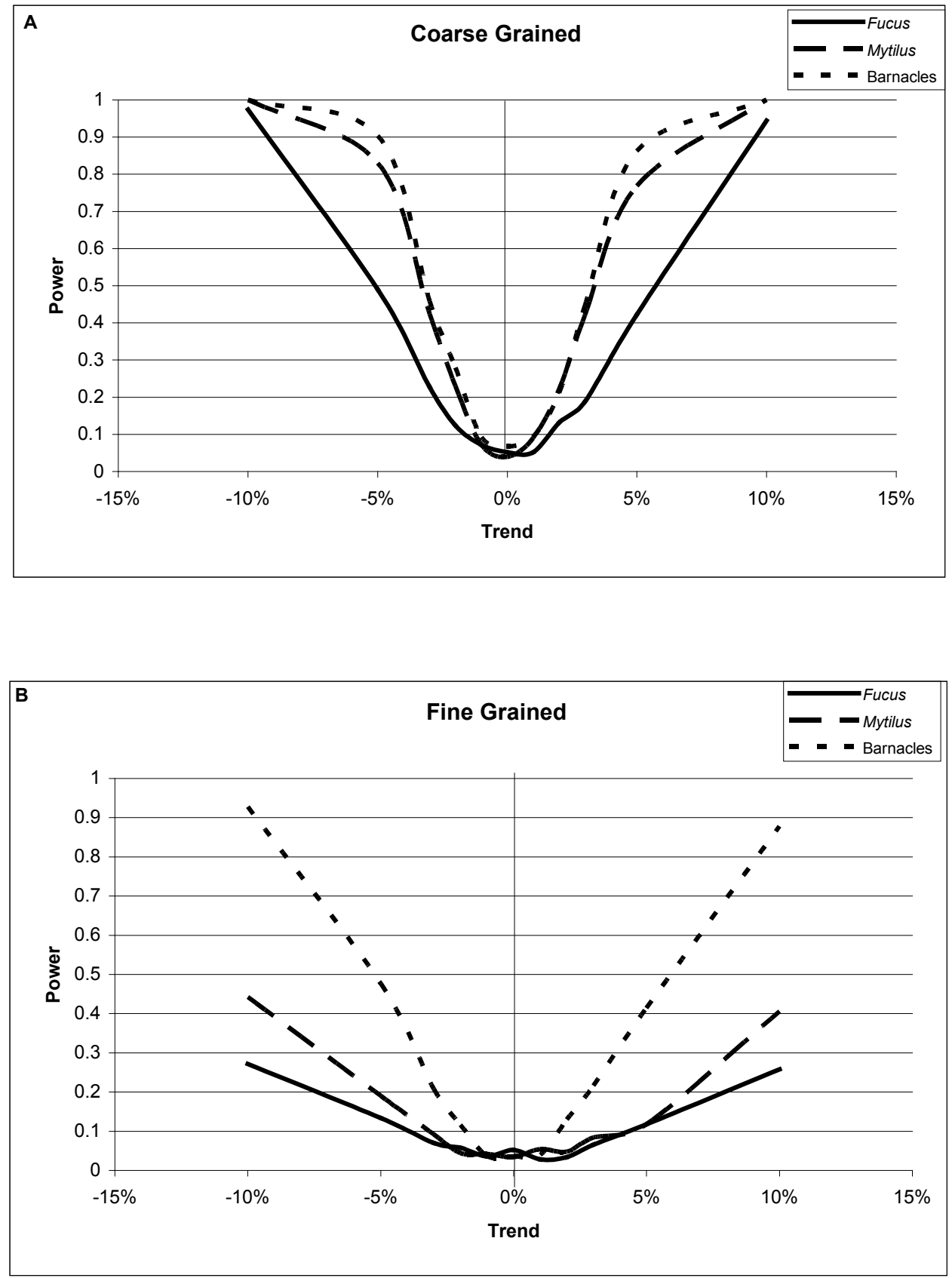

Figure 17. Comparison of the power of coarse-grained and fine-grained sampling methods to detect trends in the predominant sessile species. $\alpha=0.05$ A. Coarse-grained (1997-2001): 25 sites, 6 transects/site, 1 point/meter. B. Fine grained (1997): 6 sites, 10 transects/site, 5 points/meter. 


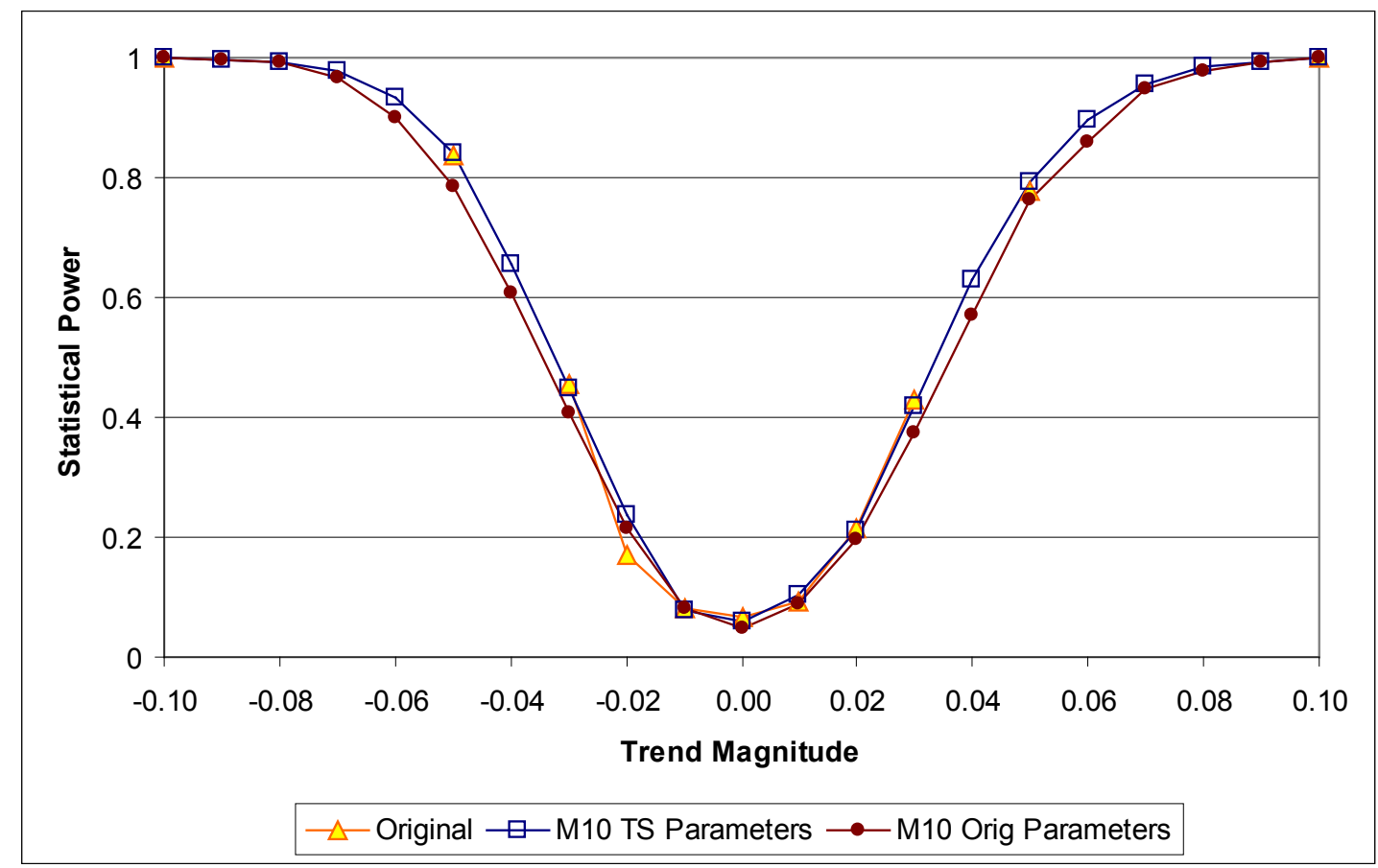

Figure 18. Statistical power comparison for Mytilus with 25 sites. Results from the original analyses are compared to those from two sets obtained with MONITOR, version 10: (1) using TerraStat parameters and (2) using the original parameters. See appendix B for more details. 


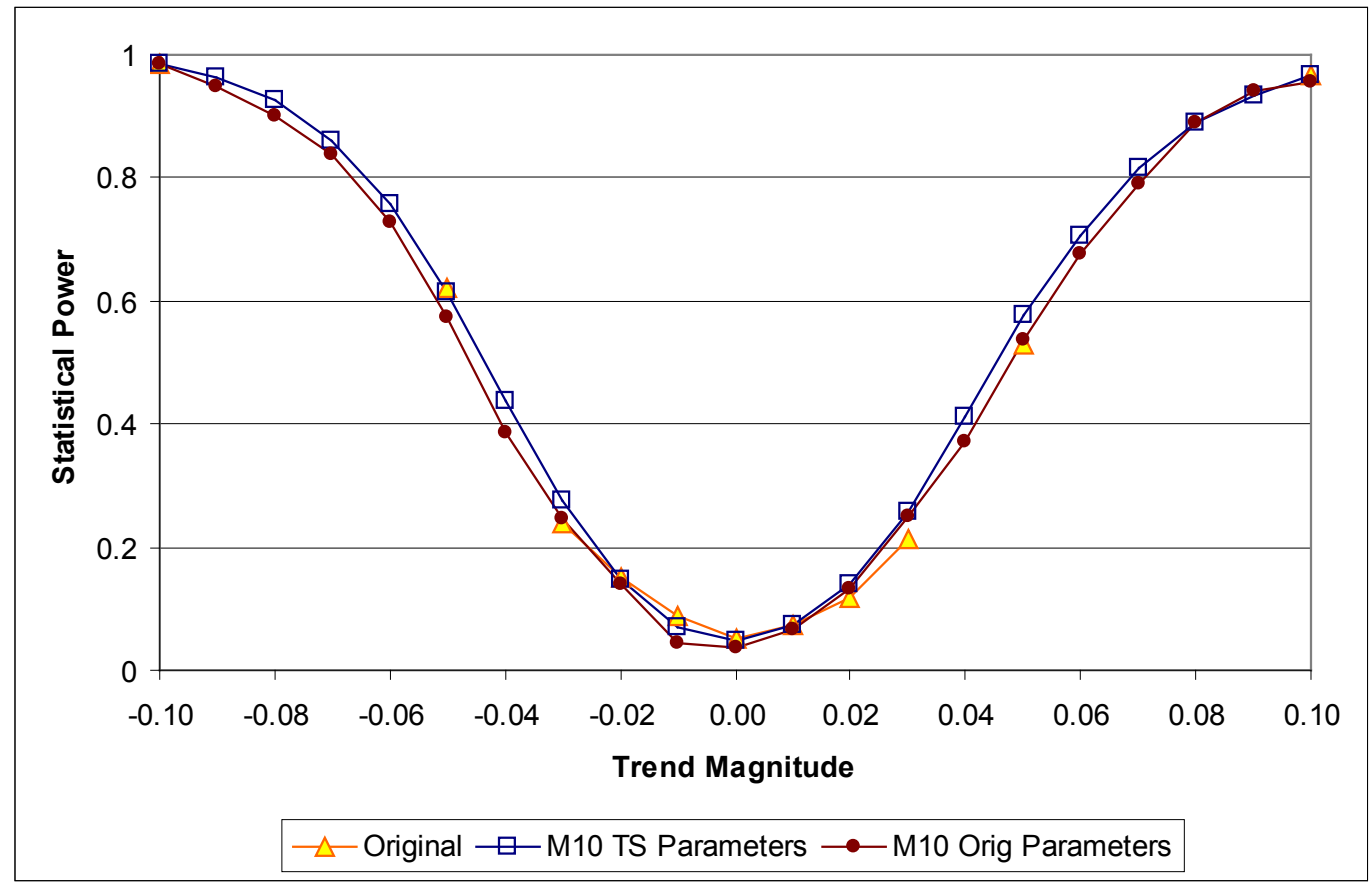

Figure 19. Statistical power comparison for Mytilus with 15 sites. See caption in figure 18 for more detail. 


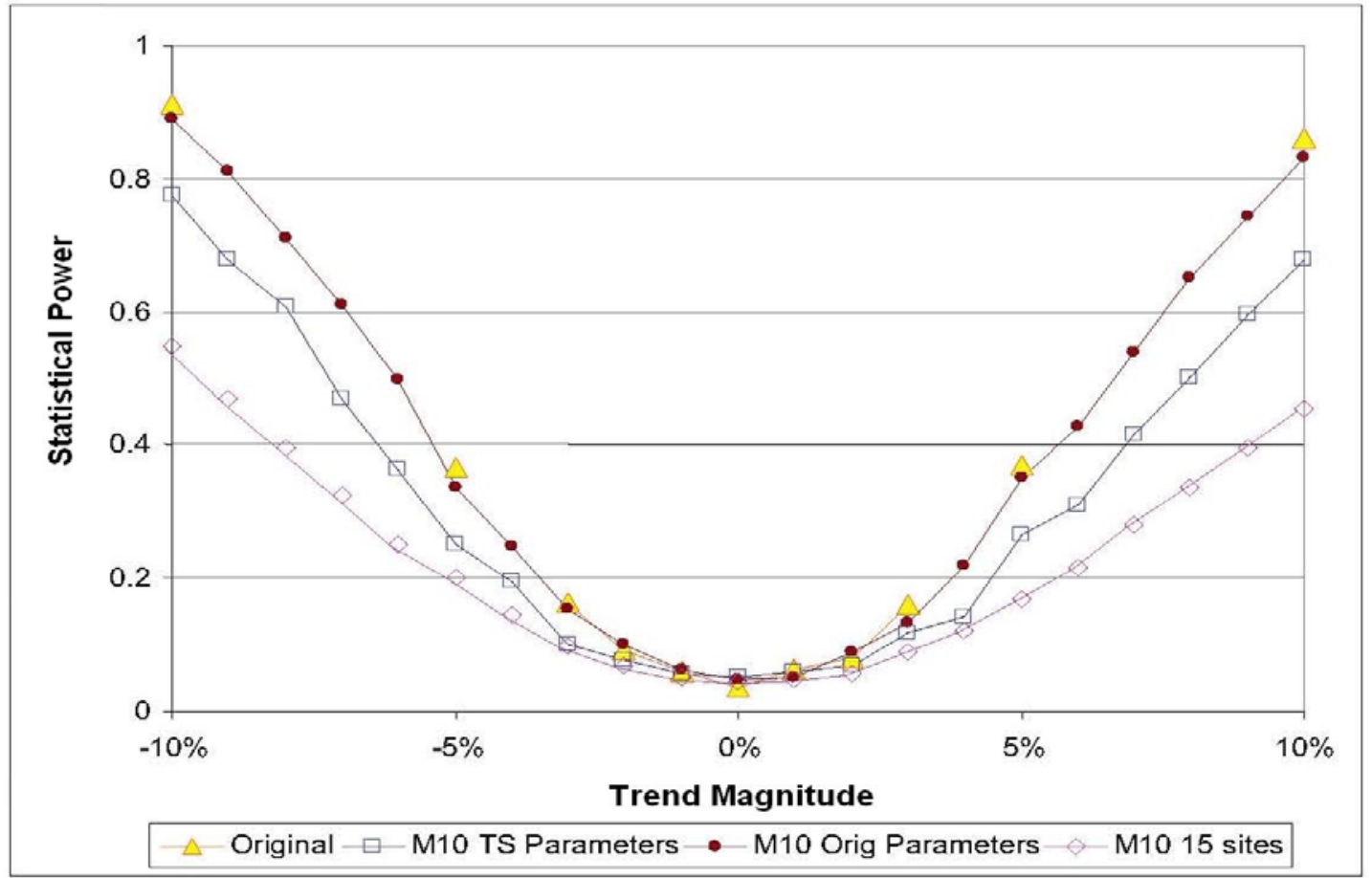

Figure 20. Statistical power comparison for Fucus. See caption figure 18 for greater detail. In addition to the three runs examining power for 25 sites, also there is a run of MONITOR 10 using data from 15 sites. 


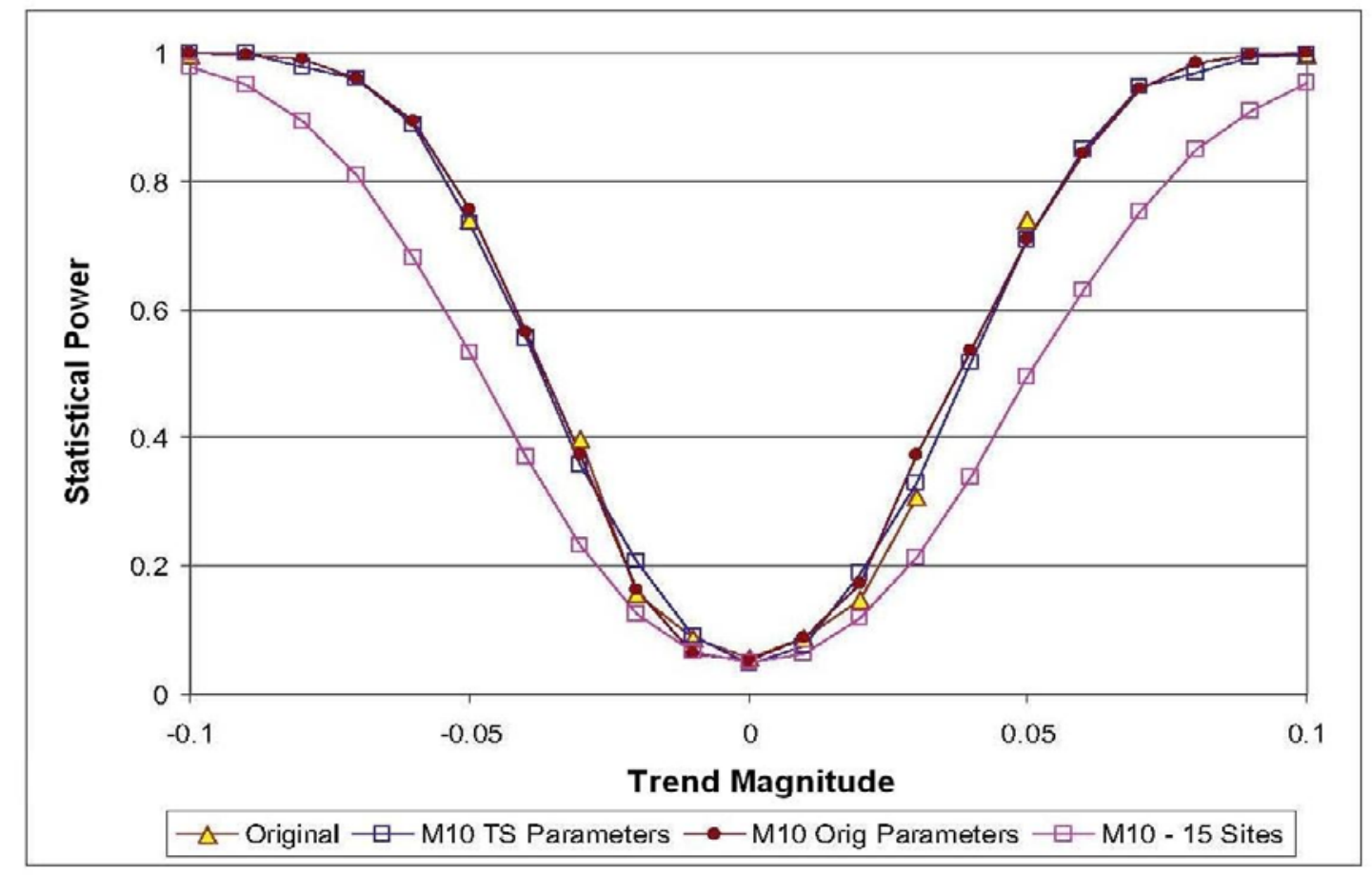

Figure 21. Statistical power comparison for barnacles. See captions for figures 18 and 20. 


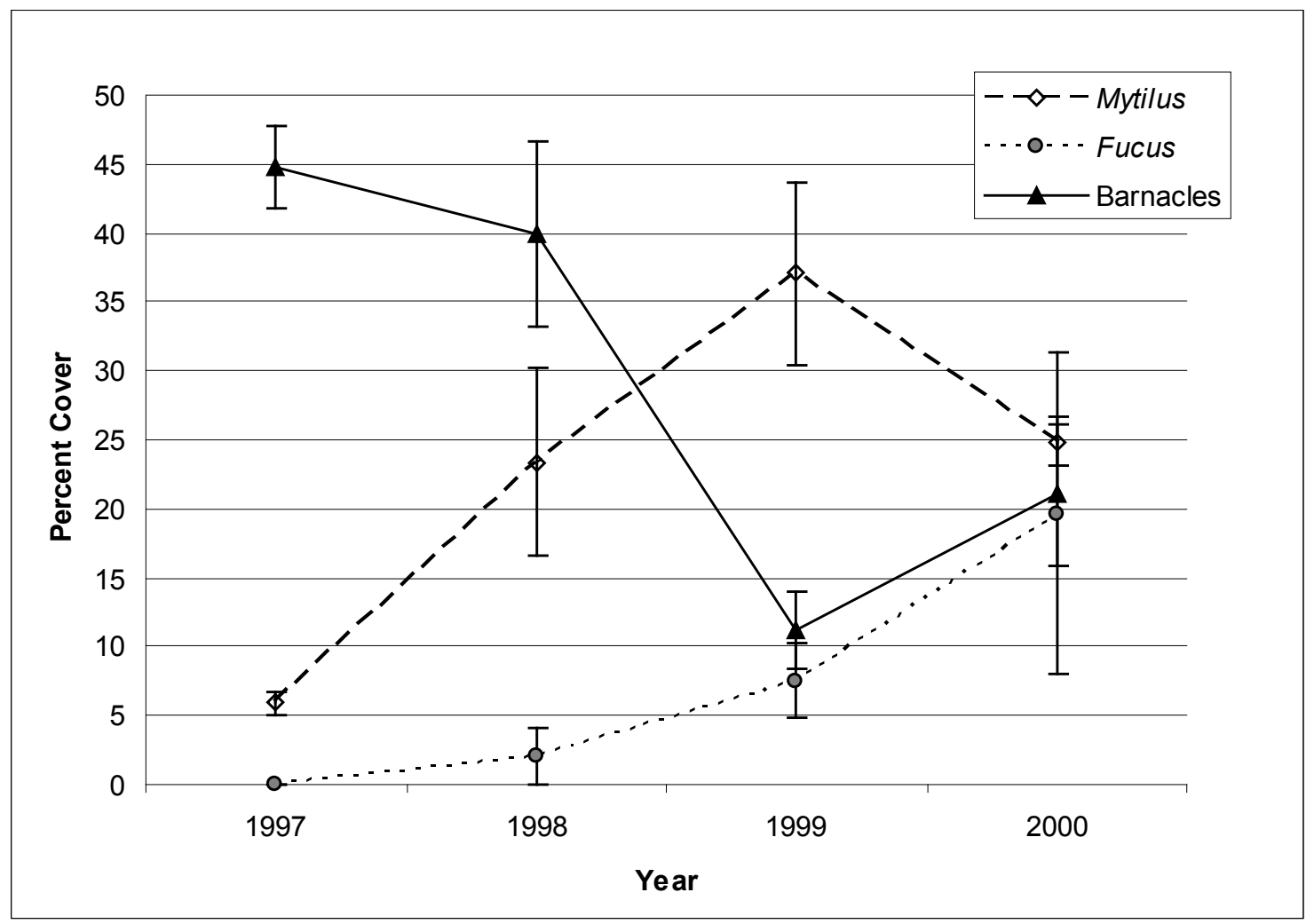

Figure 22. Mean percent cover of the predominant sessile species at Berg Bay. Data were collected in 1997-2000, from the sampling of six transects at 1 point/meter. Error bars represent \pm 1 standard error. 
Table 1. Parameters used in the power analyses.

\begin{tabular}{|c|c|}
\hline Parameter Categories & Parameter Options \\
\hline \multicolumn{2}{|l|}{ Species } \\
\hline Sessile dominants & Mytilus, Fucus, all barnacles \\
\hline \multicolumn{2}{|l|}{ Sampling Attributes } \\
\hline Sampling design & $\begin{array}{l}\text { Coarse-grained ( } 25 \text { sites; multiyear sampling; } \\
\quad 6 \text { vertical transects/site; } 1-5 \text { points } / \mathrm{m}) \\
\text { Fine-grained ( } 6 \text { sites; } 1997 \text { only; multiple methods, } \\
\quad \text { including } 10 \text { vertical transects } / \text { site, } 5 \text { points } / \mathrm{m})\end{array}$ \\
\hline Sampling method (fine-grained) & $\begin{array}{l}\text { Vertical transects }(10 / \text { site }) \\
\text { Horizontal transects }(30 / \text { site }) \\
\text { Quadrats }(30 / \text { site })\end{array}$ \\
\hline Number of sites & $\begin{array}{l}\text { Coarse grained ( } 25 \text {-site pool) } \\
\text { Fine grained (6-site pool) }\end{array}$ \\
\hline Number of transects & $\begin{array}{l}6 \text { max. for Coarse-grained sampling } \\
10 \text { max. for Fine-grained sampling }\end{array}$ \\
\hline Number of points per meter & $\begin{array}{l}1-5 \text { points } / \mathrm{m} \text { for coarse- and fine-grained } \\
\text { sampling }\end{array}$ \\
\hline \multicolumn{2}{|l|}{ Management Parameters } \\
\hline Alpha level & $0.05,0.10,0.20$ \\
\hline Level of annual change to detect & $\begin{array}{l}10 \text { percent, } 5 \text { percent, } \\
3 \text { percent }\end{array}$ \\
\hline
\end{tabular}


Table 2. The number of hits (counts) of a species or taxon during each year of sampling along the vertical transects.

[Data are from the 1998-2001 coarse-grained sampling of 25 sites, with 6 vertical transects/site sampled at 5 points/m intensity. The total number of hits can be greater than the total number of points sampled because layers of biota are recorded during the point-intercept sampling]

\begin{tabular}{|c|c|c|c|c|}
\hline \multirow{2}{*}{\multicolumn{5}{|c|}{$\begin{array}{l}\text { Taxonomic Groupings/Species } \\
\text { PLANTS }\end{array}$}} \\
\hline & & & & \\
\hline \multicolumn{5}{|l|}{ Bacillariophyta (Diatoms) } \\
\hline Diatom & 68 & 159 & 25 & 252 \\
\hline \multicolumn{5}{|l|}{ Chlorophyta (Green algae) } \\
\hline Acrosiphonia spp. & 103 & 70 & 152 & 325 \\
\hline Enteromorpha intestinalis & 73 & 44 & 38 & 155 \\
\hline Prasiola meridionalis & 10 & & 1 & 11 \\
\hline Small filamentous green alga & 40 & 112 & 149 & 301 \\
\hline Ulothrix spp. & 14 & & & 14 \\
\hline Ulvales & 573 & 894 & 1,341 & 2,808 \\
\hline \multicolumn{5}{|l|}{ Phaeophyta (Brown algae) } \\
\hline Agarum clathratum & & & 1 & 1 \\
\hline Alaria fistulosa & 1 & & & 1 \\
\hline Alaria marginata & 45 & 121 & 117 & 283 \\
\hline Alaria spp. & 195 & & 5 & 200 \\
\hline Cymathere triplicata & 1 & & & 1 \\
\hline Desmarestia spp. & & 2 & 2 & 4 \\
\hline Fine filamentous brown algae & 116 & 16 & 22 & 154 \\
\hline Fucus distichus subspecies evanescens & 6,140 & 7,222 & 6,411 & 19,773 \\
\hline Laminaria saccharina & & 1 & & 1 \\
\hline Leathesia difformis & & 2 & & 2 \\
\hline Petalonia fascia & 4 & 6 & 12 & 22 \\
\hline Scytosiphon/Melanosiphon spp. & 7 & 8 & 2 & 17 \\
\hline Small foliose brown alga & 6 & & & 6 \\
\hline Soranthera ulvoidea & 9 & 20 & 15 & 44 \\
\hline Thick brown algal crust & & & 4 & 4 \\
\hline \multicolumn{5}{|l|}{ Rhodophyta (Red algae) } \\
\hline Cryptosyphonia woodii & 17 & 22 & & 39 \\
\hline Encrusting coralline algae & 8 & 1 & 3 & 12 \\
\hline Endocladia muricata & 8 & & & 8 \\
\hline Gigartinaceae & 123 & 129 & & 252 \\
\hline Gloiopeltis furcata & 1 & & & 1 \\
\hline Halosaccion americanum & 38 & 28 & 61 & 127 \\
\hline Mastocarpus papillatus & 17 & 82 & 202 & 301 \\
\hline Neorhodamela/Odonthalia spp. & 148 & 193 & 317 & 658 \\
\hline Palmaria callophylloides & 135 & 201 & 406 & 742 \\
\hline Palmaria spp. & 223 & 337 & 231 & 791 \\
\hline Polysiphonia/Pterosiphonia spp. & 249 & 144 & 50 & 443 \\
\hline Porphyra spp. & 72 & 56 & 50 & 178 \\
\hline Red algal crust- fleshy & 3 & 7 & 2 & 12 \\
\hline
\end{tabular}




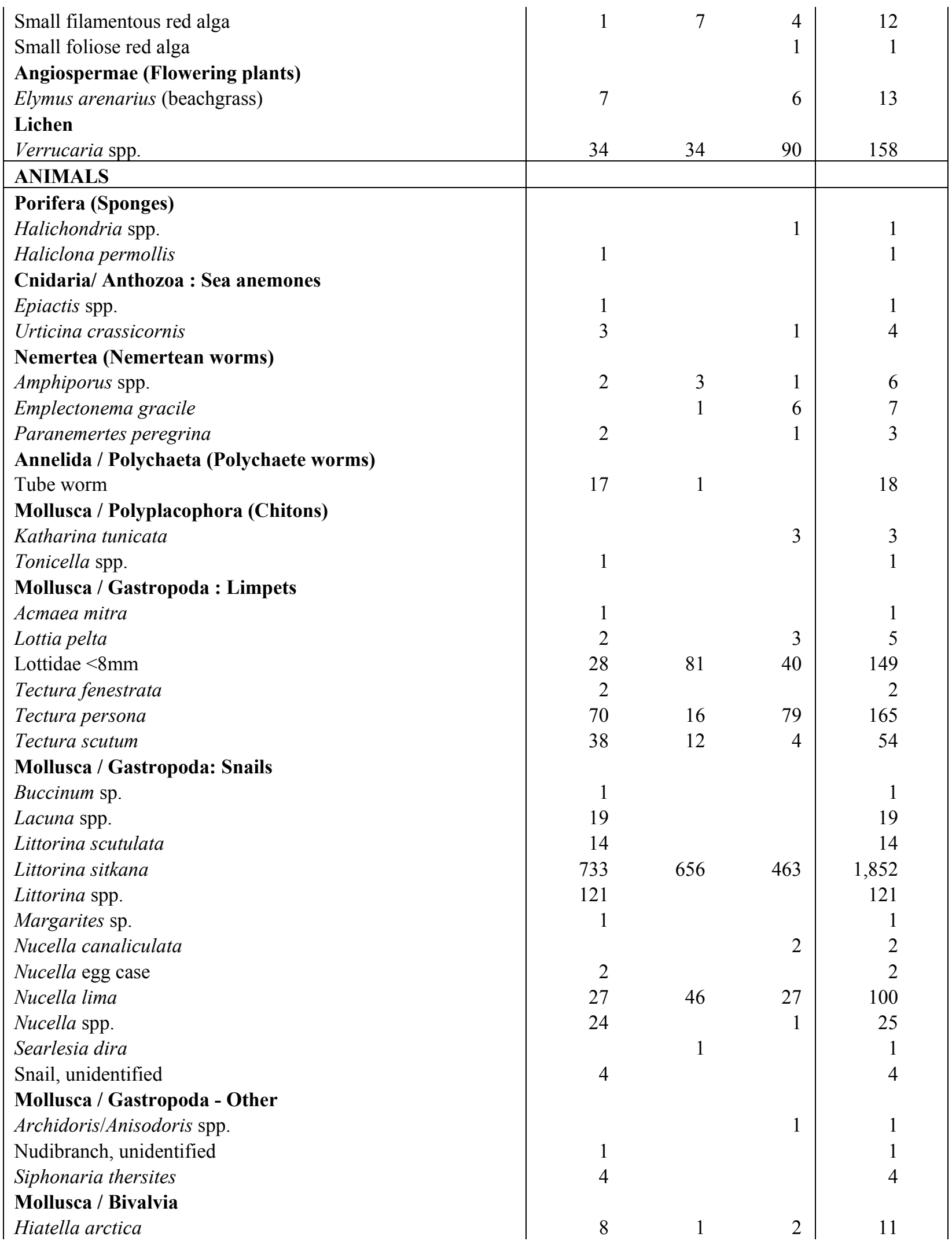


Mytilus trossulus

Arthropoda / Crustacea: Barnacles

Balanus glandula/Semibalanus balanoides

Balanomorpha

Barmacle spat/recruits $<2 \mathrm{~mm}$

Chthamalus dalli

Semibalanus cariosus

Arthropoda / Crustacea: Isopods

Isopoda

Arthropoda / Crustacea : Decapods (Crabs)

Hemigrapsus spp.

Paguridae (hermit crabs)

Echinodermata / Asteroidea (Sea stars)

Evasterias troschelli

Leptasterias epichlora

Echinodermata / Echinoidea (Sea urchins)

Strongylocentrotus droebachiensis

Echinodermata / Holothuroidea (Sea cucumbers)

Sea cucumber, unidentified

Urochordata / Ascidiacea (Tunicates)

Tunicate, solitary

Total Number of Hits

Total Number of Points Sampled

\begin{tabular}{rrr|r|}
6,244 & 4,519 & 4,140 & 14,903 \\
3,274 & 2,281 & 4,546 & 10,101 \\
1,407 & 1 & 1,475 & 2,883 \\
1,276 & 29 & 1,420 & 2,725 \\
1 & & & 1 \\
71 & 132 & 134 & 337 \\
& & & \\
2 & 2 & & 4 \\
& & & 1 \\
6 & 3 & & 9 \\
4 & & & 4 \\
3 & & 2 & 5 \\
24 & & 2 & 26 \\
19 & & & 19 \\
11 & & & 11 \\
\hline $\mathbf{1 , 9 5 8}$ & $\mathbf{1 7 , 7 0 3}$ & $\mathbf{2 2 , 0 7 5}$ & $\mathbf{6 1 , 7 3 6}$ \\
$\mathbf{2 3 , 6 1 4}$ & $\mathbf{2 0 , 8 3 0}$ & $\mathbf{2 0 , 5 1 6}$ & $\mathbf{6 4 , 9 6 0}$ \\
\hline & & &
\end{tabular}


Table 3. Trend results by site for percent cover of Mytilus.

[A linear regression was fit to log-transformed density data for each site to test for exponential trends. The trends are represented by the slopes. Significant negative (-) and positive (+) trends are indicated in the right-most column. Greater detail is in appendix B]

\begin{tabular}{|c|c|c|c|c|}
\hline $\begin{array}{l}\text { Site } \\
\text { No. }\end{array}$ & Site Name & Slope & $p$-value & Trend \\
\hline 36 & Bear Track & 0.126 & 0.472 & \\
\hline 69 & Berg Bay & 0.288 & 0.023 & + \\
\hline 63 & Between Pt Carolus \& Ripple Cove & -0.385 & 0.109 & \\
\hline 113 & Blue Mouse Cove & 0.076 & 0.273 & \\
\hline 217 & Drake Island - Lower & -0.107 & 0.111 & \\
\hline 216 & Drake Island - Mid & -0.434 & 0.001 & - \\
\hline 218 & Drake Island - Upper & -0.200 & 0.089 & \\
\hline 89 & Geikie Inlet - Lower & 0.036 & 0.747 & \\
\hline 88 & Geikie Inlet - Upper & 0.154 & 0.150 & \\
\hline 59 & Lester Island & -0.218 & 0.209 & \\
\hline 215 & Little Sturgess Island & -0.059 & 0.803 & \\
\hline 143 & Mt Abdallah Outwash & -0.112 & 0.242 & \\
\hline 12 & Muir Inlet - Lower & 0.405 & 0.002 & + \\
\hline 2 & Muir Inlet - Upper & 0.813 & 0.000 & + \\
\hline 62 & Pt Carolus & -0.167 & 0.100 & \\
\hline 151 & Rendu Inlet & 0.102 & 0.763 & \\
\hline 200 & Russel Island & -0.471 & 0.010 & - \\
\hline 108 & Scidmore Bay & -0.024 & 0.886 & \\
\hline 220 & Shag Cove & -0.135 & 0.212 & \\
\hline 214 & Sturgess Island & -0.224 & 0.177 & \\
\hline 142 & Tarr Inlet & -0.014 & 0.867 & \\
\hline 164 & Tidal Inlet - Lower & -0.071 & 0.576 & \\
\hline 163 & Tidal Inlet - Upper & -0.389 & 0.004 & - \\
\hline 223 & Willoughby Island - East & -0.282 & 0.029 & - \\
\hline 224 & Willoughby Island - West & -0.692 & 0.001 & - \\
\hline
\end{tabular}


Table 4. Trend results by site for percent cover of Fucus.

[A linear regression was fit to log-transformed density data for each site to test for exponential trends. The trends are represented by the slopes. Significant negative $(-)$ and positive $(+)$ trends are indicated in the right-most column. Greater detail is in appendix B]

\begin{tabular}{|c|c|c|c|c|}
\hline $\begin{array}{l}\text { Site } \\
\text { No. }\end{array}$ & Site & Slope & $p$-value & Trend \\
\hline 36 & Bear Track & -0.611 & 0.055 & \\
\hline 69 & Berg Bay & 1.17 & 0.000 & + \\
\hline 63 & Between Pt Carolus \& Ripple Cove & 0.641 & 0.111 & \\
\hline 113 & Blue Mouse Cove & 0.068 & 0.908 & \\
\hline 217 & Drake Island - Lower & -1.01 & 0.002 & - \\
\hline 216 & Drake Island - Mid & 0.411 & 0.047 & + \\
\hline 218 & Drake Island - Upper & 0.157 & 0.339 & \\
\hline 89 & Geikie Inlet - Lower & 0.818 & 0.001 & + \\
\hline 88 & Geikie Inlet - Upper & 0.555 & 0.015 & + \\
\hline 59 & Lester Island & 0.444 & 0.211 & \\
\hline 215 & Little Sturgess Island & 0.410 & 0.407 & \\
\hline 143 & Mt Abdallah Outwash & -0.120 & 0.564 & \\
\hline 12 & Muir Inlet - Lower & 0.152 & 0.652 & \\
\hline 2 & Muir Inlet - Upper & 0.253 & 0.451 & \\
\hline 62 & Pt Carolus & 0.317 & 0.097 & \\
\hline 151 & Rendu Inlet & 0.310 & 0.471 & \\
\hline 200 & Russel Island & -1.212 & 0.006 & - \\
\hline 108 & Scidmore Bay & 0.103 & 0.743 & \\
\hline 220 & Shag Cove & 0.107 & 0.660 & \\
\hline 214 & Sturgess Island & 0.217 & 0.510 & \\
\hline 142 & Tarr Inlet & -0.352 & 0.267 & \\
\hline 164 & Tidal Inlet - Lower & 0.421 & 0.055 & \\
\hline 163 & Tidal Inlet - Upper & -0.653 & 0.088 & \\
\hline 223 & Willoughby Island - East & -0.149 & 0.655 & \\
\hline 224 & Willoughby Island - West & 0.665 & 0.064 & \\
\hline
\end{tabular}


Table 5. Trend results by site for percent cover of barnacles.

[A linear regression was fit to log-transformed density data for each site to test for exponential trends. The trends are represented by the slopes. Significant negative (-) and positive (+) trends are indicated in the right-most column. Greater detail is in appendix B]

\begin{tabular}{|c|c|c|c|c|}
\hline $\begin{array}{l}\text { Site } \\
\text { No. }\end{array}$ & Site & Slope & $p$-value & Trend \\
\hline 36 & Bear Track & -0.011 & 0.974 & \\
\hline 69 & Berg Bay & 0.071 & 0.771 & \\
\hline 63 & Between Pt Carolus \& Ripple Cove & 0.116 & 0.713 & \\
\hline 113 & Blue Mouse Cove & 0.449 & 0.019 & + \\
\hline 217 & Drake Island - Lower & 0.002 & 0.989 & \\
\hline 216 & Drake Island - Mid & 0.097 & 0.557 & \\
\hline 218 & Drake Island - Upper & 0.093 & 0.676 & \\
\hline 89 & Geikie Inlet - Lower & -0.014 & 0.887 & \\
\hline 88 & Geikie Inlet - Upper & 0.279 & 0.333 & \\
\hline 59 & Lester Island & 0.570 & 0.057 & \\
\hline 215 & Little Sturgess Island & 0.437 & 0.026 & + \\
\hline 143 & Mt Abdallah Outwash & 0.373 & 0.004 & + \\
\hline 12 & Muir Inlet - Lower & -0.169 & 0.076 & \\
\hline 2 & Muir Inlet - Upper & 0.494 & 0.018 & + \\
\hline 62 & Pt Carolus & -0.039 & 0.899 & \\
\hline 151 & Rendu Inlet & 0.462 & 0.092 & \\
\hline 200 & Russel Island & 0.426 & 0.013 & + \\
\hline 108 & Scidmore Bay & 0.468 & 0.083 & \\
\hline 220 & Shag Cove & 0.044 & 0.668 & \\
\hline 214 & Sturgess Island & 0.007 & 0.979 & \\
\hline 142 & Tarr Inlet & 0.363 & 0.259 & \\
\hline 164 & Tidal Inlet - Lower & 0.008 & 0.962 & \\
\hline 163 & Tidal Inlet - Upper & 0.049 & 0.795 & \\
\hline 223 & Willoughby Island - East & 0.470 & 0.006 & + \\
\hline 224 & Willoughby Island - West & 0.350 & 0.022 & + \\
\hline
\end{tabular}


Table 6. Conversion of short-term trends, expressed as percent change per year, into long-term trends.

[The latter is the total percent change expected over periods ranging from 5 to 20 years, if the annual trend occurs during each of the $5,10,15$, or 20 years. In effect, this is like compounding interest, and is calculated easily, for example, -3 percent over 15 yrs $=(0.97)^{15}=0.6333$, or 100 percent -36.67 percent]

\begin{tabular}{lccc}
\hline \multirow{2}{*}{ Trend per year } & \multicolumn{3}{c}{ Short-term versus long-term trends } \\
\cline { 2 - 4 } & -5 percent & -3 percent & -2 percent \\
\hline Trend over 5 years & -22.62 percent & -14.13 percent & -9.61 percent \\
Trend over 10 years & -40.13 percent & -26.26 percent & -18.29 percent \\
Trend over 15 years & -53.67 percent & -36.67 percent & -26.14 percent \\
Trend over 20 years & -64.15 percent & -45.62 percent & -33.24 percent \\
\hline
\end{tabular}


This page left intentionally blank 


\section{APPENDIX A - Methods for the 1997 Sampling, including Aerial Survey Standard Operating Procedures}

\section{Methods for the 1997 Sampling, including Aerial Survey Standard Operating Procedures}

The Aerial Survey Standard Operating Procedures (SOP) is included here for its detail and because it was not included in Irvine (1998). Following that is a section of Methods from Irvine (1998), which concentrates on the coarse-grained (CG) and fine-grained (FG) sampling and just briefly refers to the aerial surveys. In the Methods section, note that the table and figure numbers refer to ones in the current report, unless indicated as being found only in the 1998 report. 
September 30, 1997

\author{
Standard Operating Procedures \\ for
}

AERIAL SURVEYS

for

Development of Coastal Monitoring Protocols and Process-Based Studies to Address Landscape-

Scale Variation in Coastal Communities of Glacier Bay National Park and Preserve (GLBA),

Katmai National Park and Preserve (KATM), and Wrangell-St. Elias National Park and Preserve

(WRST)

\title{
$\underline{\text { Purpose }}$
}

The purpose of monitoring programs is to detect change in populations or communities through time. If populations and communities are stable, then only an inventory of the populations or communities would be required. An inventory over large areas requires sampling to make inferences regarding populations over the area of interest. Glacier Bay proper encompasses over one thousand kilometers of coastline. As this is far too large to be thoroughly sampled, aerial surveys were carried out in order to provide a preliminary assessment of substrate and biota compositions and thus aid in selection of the sites to be sampled as part of the coarse and finegrained sampling levels of the project. The aerial surveys provided estimates of the relative abundance of different substrate types (bedrock, cobble/boulder, pebble/gravel, sand/silt/mud, coarse sand) within segments as well as biota (Fucus, mussels, barnacles). These standard operating procedures were used in 1997 when performing aerial surveys for the coastal monitoring protocol development study.

\section{Site Selection}

Within Glacier Bay National Park and Preserve the coastline of Glacier Bay proper (the inner part of Glacier Bay NP\&P, from Pt. Carolus and Pt. Gustavus northward) was divided into 200 $\mathrm{m}$ segments using ArcInfo software and a digitized coastline (CD-ROM; Geiselman, J. et al. 1997). This resulted in the identification of 5,545 segments representing a coastline of $1,109 \mathrm{~km}$. Based on our assessment that we could aerially survey 250 segments during one minus tide cycle, a random number was used to identify a starting segment, and every twenty-third segment was then systematically chosen, resulting in 250 segments. Ultimately, 241 segments were surveyed by fixed-wing airplane. Segments 173,174 , and 175 were not surveyed because they were part of a stream bank rather than intertidal areas. Segment 192 also was not an intertidal site and was not surveyed. Segments 185 and 186 were not surveyed because they were on a large mud delta criss-crossed by streams and still snow-covered. The areas matching the coordinates for segments 17 and 205 did not even vaguely resemble the segment maps from ArcInfo, thus they were not surveyed. This area of the Park has been undergoing rapid sedimentation and is now quite different than when the last charts were created. Segment 190 was not sampled because it was still covered in ice and snow. See figure A-1 for a map of the study area and segments. 


\section{Methods}

1. A map of each segment was printed on a data sheet (fig. A-2).

2. A Bellanca Scout on floats equipped with a Trimble 2000 GPS was used to fly the surveys. This is a two seat plane where the observer is seated directly behind the pilot and thus can view out of both sides of the plane. The coordinates for the segments were loaded from ArcInfo onto data cards for use with the plane=s GPS.

3. Locating the segment. Previous to the sampling we had established a technique to determine the $200 \mathrm{~m}$ segment from the air. We found that at an altitude of 150 feet, flying $300 \mathrm{~m}$ diameter circles, the distance from the point directly beneath the plane to the point beneath the center of the wingtip was $200 \mathrm{~m} \forall 15 \%$. The pilot used the Trimble to navigate to one endpoint of the segment. He would then adjust the altitude and bank into a turn and identify markers on the beach (e.g. streams, bedrock outcroppings, large boulders) to use as segment boundaries. Frequently, the location of the segment could be verified by details on the segment maps (fig. A-2).

4. Flying the segment. The aerial surveys were flown 3-8 May 1997, during minus tides. If daylight permitted, surveys were flown 2 hours before and after the low tide using the Juneau tide tables adjusted for Bartlett Cove. Because of the low altitudes and techniques employed in the surveys, we stopped flying if winds were $>10-15$ knots. Recommendations for future surveys would be to use the tide tables for the nearest tide station (Bartlett Cove, Willoughby Island, Composite Island, Muir Inlet) and sample as long as the tide is lower than 0 MLLW (mean lower low water).

Usually, the pilot flew in tight circles (diameter - 300m) at an altitude of 150-200 feet centered over the beach. In steep, constricted areas (e.g. Johns Hopkins Inlet) he would fly figure eights over the water, rather than circles. He would fly as many circles or figure eights as needed to gather the required data. Early in the surveying process, six or seven passes were needed, but this dropped to four or five by the end of the surveying period. Depending on light conditions, an "offshore" circle was flown at the minimum altitude in addition to the "above beach" circles to better view the barnacle line.

Previous to the sampling, we had familiarized ourselves with the appearances of the various substrates and biota from the air. Nevertheless, each morning early in the week of sampling and whenever we felt it necessary, we ground-truthed the data we had collected.

5. Data collection.

Slope: Upon approaching the segment, the slope category was estimated using the water surface as the horizontal, and recorded on the data sheet. Categories for slope were: 0 30E, 31-60E, 61-90E. (Note: Slope data are missing for Segment 6)

Segment area: Once the length of the $200 \mathrm{~m}$ segment had been identified, the high tide line was mentally placed by noting the edge of the Verrucaria (black lichen) zone, the snow line, the tree or grass line, or whatever else could be used to assess the line. This now created a large rectangle $200 \mathrm{~m}$ long, from the water to the high tide line. 
a. Substrate: The substrate types were determined during the approach and initial fly-over. First, substrates that were not present were marked as $0 \%$ on the data sheet. Next, substrates that were very minimal were marked $1-25 \%$. Finally, the segment rectangle was mentally broken into chunks (either vertical bands, horizontal stripes or a combination of both) and the substrates that were present were assigned percent coverages. Percent coverages of substrates should not exceed 100\%. Substrate categories were based on the sediment surface mapping protocol developed by the Glacier Bay Coastal Mapping team. The substrate categories used in the aerial surveys were: bedrock, identified as a continuous rock surface; cobble/boulder, identified as larger than tennis ball size rocks; pebble/gravel, identified by having particles smaller than tennis ball size yet still recognizable as separate particles; sand/silt/mud, identified by not having recognizable individual particles, often appearing as one continuous surface; and coarse sand. Usually, silt/mud could be identified separately from sand because it became deeply channeled, had many colorful algae growing on it, and seeped out thus clouding the water. (Note: substrate percent coverages are missing for Segment 197, though substrates present are listed).

b. Biota: During approach and substrate determination, a preliminary scan for Fucus, mussels, and barnacles was made. After substrate determinations were finished, any species that was not present was marked as $0 \%$. Next, species that were very minimal were marked $1-25 \%$. Finally, the segment rectangle was mentally broken into chunks (either vertical bands, horizontal stripes or a combination of both) and the species that were present were assigned percent coverages. Total percent coverages of biota on a segment ranged from $<100 \%$ to $>100 \%$. Fucus, growing in dense beds down to small clumps, was readily identifiable from the air. If mussels were present as a continuous band they were easy to identify. If mussels were patchy or covered with barnacles or barnacle spat, they were more difficult to identify from the air. The surest method for recognizing mussels was to scan the sides of any boulders or larger cobbles. Mussels, even if covered by barnacle spat, contrasted with the surface of the larger rocks. On segments comprised of pebble/gravel, mussels were extremely difficult to recognize and were possibly underestimated. The barnacle zone was recognizable by its distinctive greyish-chalky color. The pilot usually had to experiment with altitude and distance offshore to bring this color separation into view. If barnacles were patchy or the segment was pebble/gravel, barnacles were more difficult to recognize and may have been underestimated. Several other biotic groups were occasionally visible and recognizable, although not widespread throughout the park: encrusting red algae, green algae (individual species could not be differentiated from the air), subtidal brown algae (e.g. Alaria), subtidal urchin barrens, and sea stars. These were noted on the data sheets but no estimates of percent coverage were given.

\section{References}

Geiselman, J., Dunlap, J., Hooge, P., and D. Albert. (Eds.). 1997. Glacier Bay Ecosystem GIS CD-ROM set. U.S. Geological Survey and Interrain Pacific. Anchorage and Juneau, Alaska. Authors? 1997. Sediment surface mapping protocol for Glacier Bay Coastal Mapping, Coastal Monitoring, and Benthic Surveys. (sedprot.w51) 


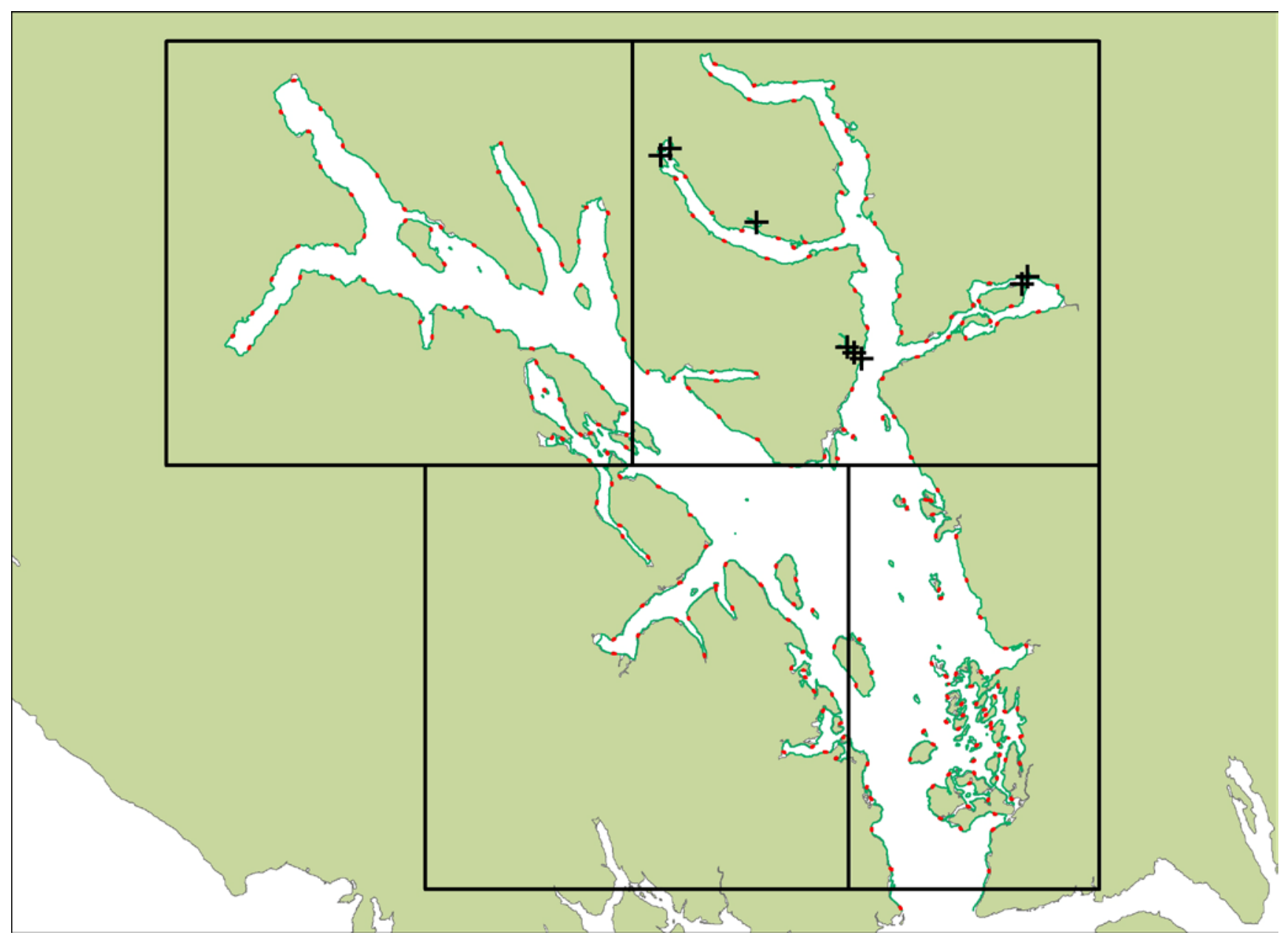

Figure A-1. Map of the study area showing Glacier Bay proper. Each red dot represents one of the $200 \mathrm{~m}$ segments sampled during the 1997 aerial survey. The eight "X" marks show sites that were not sampled. 


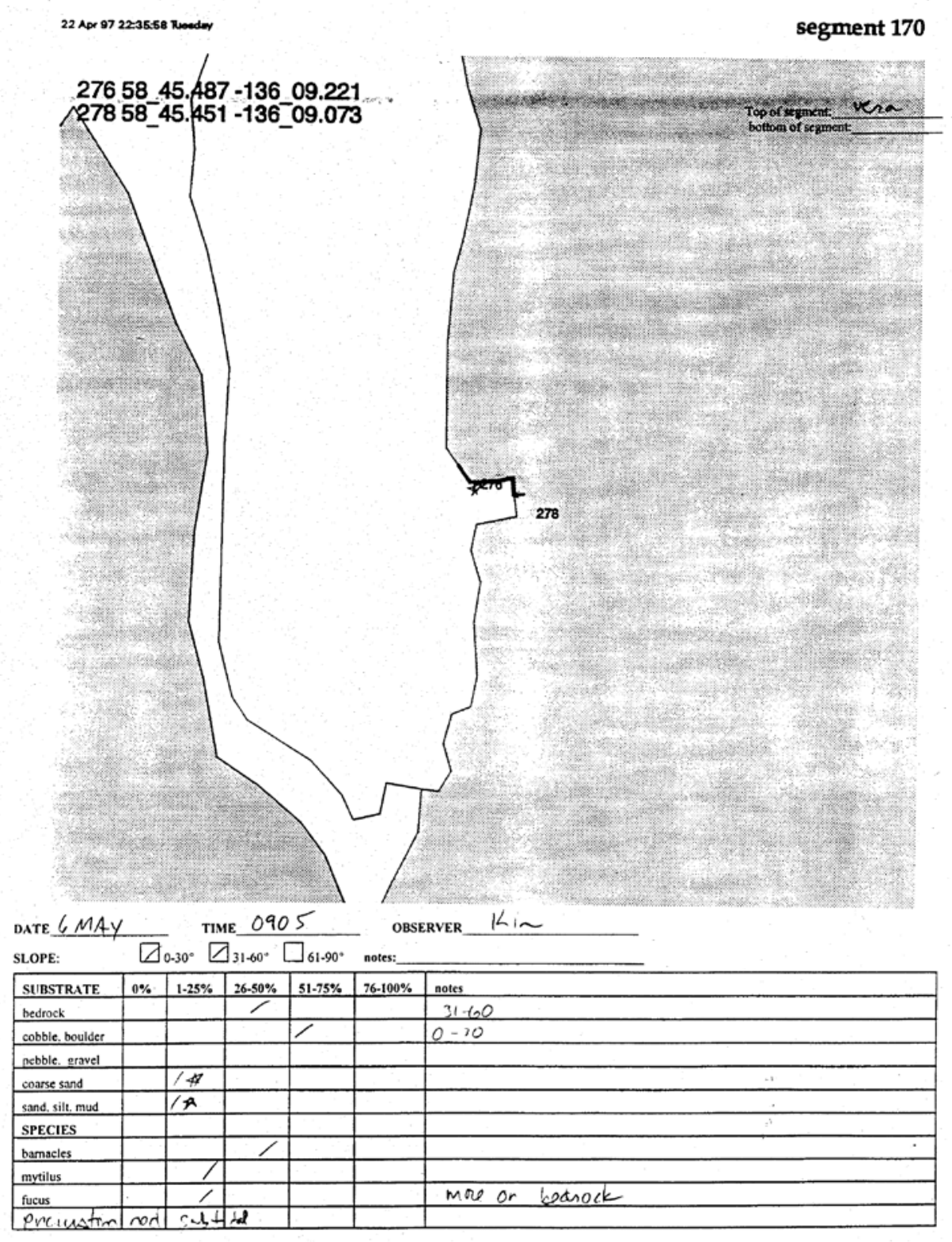

Figure A-2. An example of a completed data sheet/segment map from the aerial surveys. The thickened line delineates the $200 \mathrm{~m}$ segment. The numbers 276 and 278 are the endpoints corresponding to the GPS coordinates given in the upper left corner. 


\section{Methods (from Irvine, 1998)}

\section{Objective 2: Sampling Design}

Methods used in the three sampling designs [a. broad-scale inferential, b. nested inferential (=coarse-grained), and c. nested, intensive (=fine-grained)] are detailed in the preceding Aerial Survey SOP and below. The first level (a) used aerial surveys, while (b) and (c) used on-site sampling of differing degrees of intensity. Figure numbers in this section refer to figures within the main body of the report.

\section{A. Broad-scale, inferential (= Aerial surveys). See Aerial Survey SOP (preceding this section)}

\section{B. Nested inferential (selected habitat, coarse-grained).}

Under the nested, coarse-grained category, a smaller and more selected set of sites was sampled at a greater level of detail than the broad aerial categorization of the coast accomplished under (A) above. The first decision was to select a habitat type for examination. Stratification by habitat type should reduce the level of variability of species abundances. As mentioned above, we initially had been asked by the park to study protected rocky (bedrock) habitat. However, only 3 of the 241 segments surveyed by air were comprised of $76 \%$ or greater bedrock, with a slope $<60$ degrees. At that point, we had the option to: (1) conduct more aerial surveys to increase the number of segments of the targeted habitat type, (2) relax the habitat parameters to include both bedrock and a more predominant habitat type, or (3) change the habitat choice altogether. We decided on the basis of further discussions with Center staff (including Mark Udevitz) and the Park staff, to relax the habitat parameters and include unconsolidated substrates (cobble/boulder) as a target of this study. All those segments with bedrock ( $\geq 1 \%)$ and/or 76 $100 \%$ cobble/boulder were included.

This change in targeted habitats resulted in a larger pool (111) of the original 241 segments being considered for further study. Thirty segments were randomly selected for sampling. However, several selected segments had to be deleted because they were too steep, had no land exposed at high tide, or were in wildlife protection areas where no access was allowed. Twenty-five sites (fig. 2) were ultimately sampled (Protocol for Coarse-Grained Sampling).

Coarse-grained surveys were undertaken at this selected subset of protected bedrock and cobble/boulder sites. During surveys we: (1) verified the substrate composition of the segment and its associated assemblage of species, (2) laid out 6 line transects running parallel to the elevational gradient (vertical transects) from mean high high water (MHHW) to the 0 foot tide level ( mean low low water, MLLW), (3) sampled a systematic set of points along the transects (1 point $/ \mathrm{m}$ in 1997; $5 \mathrm{pts} / \mathrm{m}$ in later years) to indicate zonation as well as the relative abundance of species along the transect tape, (4) sampled band transects along the transect line (1 m to each side) for larger organisms (starfish, sea urchins, and large chitons), (5) conducted a CTD (conductivity, temperature depth) cast off of each site, and (6) photographed the site (still shots).

The sampling area of the segment was demarcated at its upper side by a horizontal segment line laid at the lower edge of the Verrucaria (black lichen) zone, defined as approximately $20 \%$ coverage of Verrucaria, usually close to the juncture of the barnacle and Verrucaria zones. On some beaches, the beach wrack and/or beach grass were used as indicators 
of the highest tide levels. The horizontal segment line was laid parallel with the shoreline. Six line transects were laid out parallel to the elevational gradient ("vertical") and perpendicular to the shoreline, running from the horizontal segment line down to mean MLLW. The 0' tide level was determined by using the Tides and Currents computer program (Nautical Software, Inc.) for the sampling date and nearest tide station location (Bartlett Cove, Willoughby Island, Composite Island, or Muir Inlet). The start of the first vertical transect line was determined randomly within the first $33 \mathrm{~m}$ of the horizontal segment line; the succeeding transect lines were laid out systematically with respect to the first. One point was sampled at each meter interval of the vertical transects $(0,1,2 \mathrm{~m}$, etc.): Note: at some sites sampling was increased in density, and in later years, sampling was conducted at 5 points $/ \mathrm{m}$. Point sampling was 3 -dimensional; all species underlying the point were recorded, from top to bottom, and substrate underlying the point was also recorded.

We expected to sample two sites per low tide, six days of low tides per low tide cycle, for two low tide cycles, giving 24 total expected sites sampled, however, the sites were spaced sufficiently far apart that usually only one site could be sampled on a tide. Data obtained were the percent coverage of sampled species, densities of species counted in the swath surveys, and measures of zonation as reflected in the sampling of the vertical transects.

Initial sampling in May at a site (\#63) with very long transects (up to 140m) caused a reexamination of the intensity of sampling that was possible at this level of the survey. Although we had planned originally to sample every $20 \mathrm{~cm}$, we had to scale the sampling to one point per meter in order to be able to complete the sampling of 6 transects by 3 people in one tide. So the one point sampled per meter of transect length became the standard for all coarse-grained level sampling. When we moved up bay and found the transects were considerably shorter, we increased the density of sampling (in order to increase the biological information on a site), but analyses using 1997 data, except where noted, compare all coarse-grained sites at the standard level of sampling (one point/meter).

\section{Intensive sampling (selected habitat, fine-grained)}

The shift in habitats focused on in Level B (the coarse-grained surveys) had consequences for the selection of sites for Level $\mathrm{C}$ (the fine-grained, intensive sampling). The lack of bedrock-dominated segments with slopes less than 60 degrees in the aerial surveys prompted relaxation of the habitat parameters, which resulted in a mixed habitat type becoming the focus in Level B. The 25 Level B sites contained bedrock and/or cobble/boulder substrates, with the mix ranging from $76 \%$ or greater bedrock with $1-25 \%$ cobble/boulder to $76 \%$ or greater cobble/boulder with no bedrock. These sites had been drawn from the pool of all sites containing bedrock, plus all $76 \%$ or greater cobble/boulder sites; these latter did not have to have a bedrock component.

The methods of selecting and sampling the sites affect the ability of the park to generalize from the selected sites to other similar sites. We planned to sample six sites at this level. Two factors weighed in narrowing the focus of sites and habitats selected for fine-grained sampling. First, in light of the variability in the habitat mix of the coarse-grained sites, we decided, in consultation with Center and Park staff to concentrate efforts on predominately cobble/boulder sites. In part, the park advocated for consideration of this habitat type (or subset of Level B habitat mix) because of its greater abundance within Glacier Bay proper than low-angle bedrock sites. Only those sites with $76 \%$ or greater cobble/boulder were considered for inclusion. Having 
a bedrock component did not eliminate a site. For example, Site 69 was included, and it contained both $76 \%$ or greater cobble/boulder and $1-25 \%$ bedrock. Second, we decided to narrow the geographical scope of the sites sampled in this third level, to sites within a region readily accessible from Bartlett Cove by skiff, in order to allow for frequent access. Thus the region from just south of the two arms to approximately the Bartlett Cove level was chosen. Two methods of maintaining inference to the broader pool of sites were apparent. One, to choose a subset of the sites from level B that fit the parameters described above, or two, to draw a new pool of sites from Level A that fit the parameters. We decided to choose a subset of those from level $\mathrm{B}$, in part because we then had the advantage of comparing the two types of sampling at the same sites. In total, below the juncture of the two arms in Glacier Bay proper, were eight sites that had been defined as predominately cobble/boulder by the aerial surveys. One of those in Geike was discarded because the level B surveys had revealed that little cobble/boulder was present. The most southerly geographic outlier was also dropped. This resulted in the selection of six sites that ranged from just south of the two arms to slightly south (but across bay) of Bartlett Cove (fig. 2). The data from these sites is applicable to the range of cobble/boulder habitat within the band or region that the sites occupy, as these sites were originally randomly selected from a pool of the selected habitat type (Level B).

Within-site sampling

All of these sites had been sampled under the coarse-grained regime. The sampling at the fine-grained level included sampling that was similar to the coarse-grained level, although more intensive, plus additional methods of sampling. Locating the site and delineating the horizontal segment tape were accomplished in the same manner as under the coarse-grained procedures.

The within-site sampling included:

1. vertical transects,

2. horizontal transects,

3. band sampling of both vertical and horizontal transects,

4. quadrat sampling, both point-contact and counts of small mobile invertebrates

5. mussel sampling, and

6. fixed quadrats (at one site),

7. photographs of the various sampling units, and

8. some additional marking of the site.

The layout of the sampling is depicted in figure 3.

\section{Vertical transects:}

Line transects (vertical transects) were laid out as under the coarse-grained protocol, except 10 instead of 6 were laid out. These transects were positioned parallel to the elevational gradient of the segment, running from the horizontal segment line down to MLLW. The start of the first vertical transect along the horizontal segment tape was determined by choosing a random number between 0 and 20, and the remaining transects were placed systematically relative to the first. Transect lines were draped so as to generally conform to the substrate, rather than being stretched taut. The intention was that all exposed surfaces had an equal probability of being sampled. 
Point sampling of Vertical (and Horizontal) Transects: Systematically selected points along the vertical transects were sampled to provide estimates of the relative percent cover of biota along the transects, as well as the relative percent cover of underlying substrates. This sampling of points also provides information on the zonation of species. At fine-grained sites, five points were sampled per meter (each $20 \mathrm{~cm}$ ).

Points were sampled 3-dimensionally, noting the species and substrate under each point. The right edge of the tape and the distance hash marks along the tape were used as cross hairs to mark the point. We used knitting needles to facilitate the 3D sampling of the naturally heterogeneous topography for all types of point sampling on the fine-grained level (vertical and horizontal transects, and point-contact quadrats). The knitting needle was used to follow the point perpendicularly from the tape to the substrate. All species underlying the point were recorded, in order from the top down, including multiple "hits" or layers of the same species. The substrate, which also could be multi-layered, was recorded. Organisms were identified to the lowest taxonomic level possible in field sampling. Some taxa are grouped because they could not be discriminated in the field readily. Substrates were classified according to a modified Wentworth-scale (appendix D1.2, this report).

\section{Horizontal transects:}

(a). Determining the elevations at which horizontal transects and quadrats are set. As set out in the initial protocol, the total tidal range of a site was going to be determined by assessing the angle of the slope, and multiplying the sine of that angle by the length of the transect (the hypotenuse). We planned to define three elevational zones by dividing the tidal range into thirds. A random number was then going to be used as a multiplier to determine the point within each vertical zone to be sampled, so that each zone was sampled at the same proportionate elevation. The same random number $(0.71)$ was applied to all six sites sampled this round in order to facilitate comparisons between sites. This number set the sampling locations fairly high within each zone.

After determining the tidal heights for sampling along each transect, the actual locations would have been determined through use of the tide programs. Although we initially set up our calculations at Site 63 in this manner, we could not follow the procedure because the high tides were not going to be high enough for us to locate, via the tide tables, the actual locations of the upper horizontal transects. Thus, we modified the procedure in the field, and decided that we had to assume an equal slope and use the distance of the transect line as a proxy of the elevational gradient. So, in the field, the transect length was divided into thirds: a low, middle, and high portion, and a random number (0.71) was multiplied by the length of each third to determine the location for placement of the horizontal transects. For example, if the transect length was $45 \mathrm{~m}$, each $1 / 3$ segment equals $15 \mathrm{~m}$, and the sampling location within each third is the random number (0.71) times $15 \mathrm{~m}=10.65 \mathrm{~m}$ from the lower end of that third of the transect. This same method was used for all sampling of fine-grained sites in 1997. 
(b). Laying and sampling horizontal transects. Horizontal transects were $10 \mathrm{~m}$ in length, with the origin at the vertical tape, and the horizontal tape laid to the right as one faces shore. They were run parallel with the water's edge, unless they were constrained in other ways. For example at site 69 , where the bedrock cliff jutted out and made sampling a line parallel with the water impossible, then the transect line was run parallel with the segment line. Point sampling was conducted in the same manner and at the same intensity as for the vertical transects. The sampling under each point was 3D, and was recorded as for the vertical transects.

\section{Band sampling:}

Band surveys were conducted along both vertical and horizontal transects as was done in the coarse-grained sampling. A band one meter to each side (total width of 2 meters) of the transect tape was surveyed for the presence and abundance of specific species, primarily mobile species or larger, rarer species unlikely to be sampled well by point sampling (band species were identified on the species identification list). The number of each band species observed per meter was recorded in the appropriate place on the data sheet. If no band species were observed, that was noted. During the band surveys, substrate was not disturbed, therefore species that more commonly are found in the crevices between cobbles/boulders or under them were probably under-represented (e.g., the six-armed starfish, Leptasterias hexactis).

At some sites, a dense Fucus zone made it difficult to observe band species. In these cases, the bands were scanned from 0 MLLW to the bottom of the Fucus zone (this was noted on the data sheet as well as the distances along the transect line that were searched). In most cases, the Fucus zone was scanned, but Fucus was not moved if it was dense. In 1997, dense Fucus occurred at only a few sites, primarily in the lower bay area.

\section{Quadrat sampling $(33.3 \mathrm{~cm} \times 33.3 \mathrm{~cm}$ quads):}

Quadrats used were $1 / 9 \mathrm{~m}^{2}$, with an inner diameter of $33.3 \mathrm{~cm}$ per side. A grid of lines was set across the quadrat frames (see fig. 4), and the 36 intersections of the grid created the sampling points. Quadrats were laid out in a systematic manner at the interstices of the vertical and horizontal transects, with the lower edge of the quadrat set along the upper side of the horizontal transect, and the left edge to the right of and along the vertical transect. The quadrat frame was allowed to lie naturally relative to the slope and heterogeneity of the substrate. Occasionally, a quadrat was unstable and had to be supported in order to be assessed. Once the quadrat frame was set, points were sampled perpendicular to the plane of the quadrat. Points were assessed on substrates with slopes up to 90 degrees; substrates where the angle was $>90$ degrees (underhangs) were ignored. Sampling of points was done in a 3-dimensional manner as for the vertical and horizontal transects. The 3-dimensional assessment of points included counting multiple layers of biota (of the same or different species), and multiple substrates if they were discernible.

Prior to sampling, photos were taken of both the regular quadrat and the adjacent mussel counting quadrat (see below), as the sampling could disturb the position of biota within the quadrat. Usually, a single photo of both the quadrat and adjacent mussel count quadrat was taken. However, if the quadrats lay at different angles separate photos were taken because one photo couldn't be taken normal (perpendicular) to both. 
After assessing the point contacts, counts were made of mobile species inside the quadrat, including: chitons, limpets, Nucella spp. (predatory gastropods), nemerteans, sea urchins, and starfish. Counts of mobile species were done to the lowest species distinctions. Limpets $<8 \mathrm{~mm}$ were lumped into Lottiidae, due to difficulties of accurately identifying small limpets (Nora Foster, pers. comm.). In addition to counts of mobile species, the quadrat was scanned for the presence of other sessile species not counted under the points (including species like Mytilus, whose abundance was highly variable within quadrats). These species were listed as present. Mytilus was not uniformly scanned for presence in 1997.

A small subquadrat $(10 \times 10 \mathrm{~cm})$ set within the upper left of the $33.3 \mathrm{~cm} \times 33.3 \mathrm{~cm}$ quadrat was used to assess the density of littorine snails. In 1997, Littorina scutulata was noted by one observer to be present only at Site 59; L. sitkana having been the predominant species observed. Other observers either saw only L. sitkana, or did not discriminate species.

Barnacle spat. The density of barnacle spat was assessed in the upper left quadrant $(5 \times 5 \mathrm{~cm})$ of the subquadrat $(10 \times 10 \mathrm{~cm})$ in which littorines were counted. For 1997, barnacle spat for the quadrat sampling within the fine-grained sampling, which took place late July to August, was defined as barnacles from 0-2 $\mathrm{mm}$ diameter. In the coarse-grained sampling, which took place in June to mid-July, barnacle spat was considered $0-1 \mathrm{~mm}$.

\section{Mussel quadrat sampling:}

There were two types of mussel quadrat sampling: (a) sampling within the mussel zone along each vertical transect (with collection of mussels and Fucus), and (b) non-disruptive sampling set systematically along the vertical transects. In both types of mussel sampling, a square $500 \mathrm{~cm}^{2}$ quadrat was used.

(a). Sampling within the mussel zone followed the layout and sampling procedure of the Exxon Valdez oil spill Nearshore Vertebrate Predator (NVP) Project mussel sampling, with one minor placement deviation noted below. Briefly, the length and location of the mussel zone was defined along each vertical transect, and a random number from $0-1.0$ was used as a multiplier to establish the location of the lower horizontal edge of the quadrat within the mussel zone. The random number was multiplied by the length of the mussel zone, then the number was added to the upper edge of the mussel zone to determine the quadrat location relative to the distance down the vertical transect. In our sampling, the $500 \mathrm{~cm}^{2}$ quadrat was displaced one quadrat width to the left of the vertical transect line, so as to not interfere with the second type of mussel quadrat sampling, in which the quadrat was placed adjacent to the vertical transect line (at three locations along each line). One quadrat was sampled per vertical transect line under the first type of mussel sampling. In these quadrats, all mussels and Fucus were collected from the quadrat, placed into a labeled plastic bag, and frozen upon return to the ship, or kept cooled until returned from the field. While removing the mussels and Fucus, if shape of the substrate allowed, then all surfaces were scraped of biota in order to be able to use these clearings to follow recruitment and succession. Back in the lab, mussels were measured, and the length and reproductive status of Fucus plants were recorded. 
(b). Systematic sampling along the vertical transect lines involved non-destructive sampling of $500 \mathrm{~cm}^{2}$ quadrats laid out systematically at three points along each vertical transect line. The quadrats were laid out at the intersections of the vertical and horizontal transects, on the left hand side of the vertical transect line, as one faces shoreward. The lower right hand corner of the 500 $\mathrm{cm}^{2}$ quadrat is opposed, across the transect line, from the lower left hand corner of the $33.3 \mathrm{~cm} \times$ $33.3 \mathrm{~cm}$ quadrat, and both have their lower edge at the elevational level defined by the horizontal transect (fig. 3). As mentioned above in the discussion of the $33.3 \mathrm{~cm} \times 33.3 \mathrm{~cm}$ quadrat sampling, photos were taken of both quadrats before they were assessed. Three quadrats were placed along each vertical transect line, for a total number of quadrats sampled across the site of 30 . Within these quadrats, mussels were counted, but not collected and substrates were not disturbed. Because mussels were sometimes very dense, the quadrat frame was subdivided into three sections with line to facilitate counting, and tally counters were used. Any mussel even partially within the quadrat frame was counted, irrespective of the amount of its shell in the quadrat. All mussels that could be observed without disturbing substrates within the quadrat were counted, including ones in crevices or beneath underhangs, and the smallest identifiable mussels.

\section{Fixed quadrats:}

In 1997, time constraints and design challenges led to fixed quadrats being set up and sampled only at Site 69 . Ten fixed quadrats $(33.3 \mathrm{~cm} \times 33.3 \mathrm{~cm})$ were laid out and marked on boulders of sufficient size. At this site, the boulders were selected arbitrarily and were spread over the length $\mathrm{f}$ the site. These quadrats were sampled in the same manner as the other main quadrat sampling on the beach: 36 points assessed, counts of small mobile invertebrates, subsample counts of littorines and barnacle spat, and scans for other sessile species. Photographs of quadrats are also taken. 
This page left intentionally blank 
APPENDIX B - TerraStat Report 


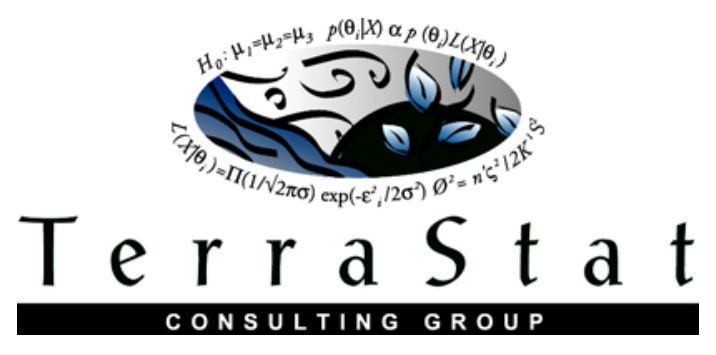

Review of

"Development of Coastal Monitoring Protocols and Process-Based Studies to Address LandscapeScale Variation in Coastal Communities of Glacier Bay National Park and Preserve: Phase II: Development and testing of monitoring protocols for selected intertidal habitats and assemblages (Draft Final Report; 2002)"

\author{
By Alice Shelly \\ TerraStat Consulting \\ 5002 Lodge View Lane \\ Austin, TX 78731 \\ alice@blarg.net \\ May 14, 2007
}

This report summarizes my review of the power analysis detailed in your 2002 draft final coastal monitoring report for Glacier Bay. Overall, it seems that the power analysis was adequate with reasonable results. Although there were some estimation methods that were not ideal, I have now verified (using the updated program MONITOR 10.0) that these differences did not significantly affect the power estimation for mussels (Mytilus trossulus, MY) and barnacles (BARN). The statistical power for rockweed (Fucus gardneri, FU) may have been overestimated in the coastal monitoring report by $5-10 \%$. There remains some uncertainty in the results due to the assumption of lognormal errors, which is not ideal for MY and BARN. The only way to address this uncertainty would be with an independent simulation program which would allow for simulations from empirical error distributions or a more suitable fitted distribution.

With multiple sites, program MONITOR estimates statistical power for a trend monitoring program assuming an analysis using route regression. Route regression consists of a linear or exponential regression relating years to the estimated percent cover for each site. The slope of the regression line for each site is the estimated trend for that site, and the mean of these slopes is tested for differences from zero using a t-test. To estimate power, the user inputs site means and variances. MONITOR simulates normal or log-normal residuals around projected linear or exponential trend lines as specified by the user. Variability among site trends is an optional feature. In version 10.0, the variance can also be partitioned into variance among years and variance within years (although this feature is not yet functioning properly per James Gibbs 4/3/07). 
For the Glacier Bay power analysis, the questions of interest are:

1) Given the variability observed over the sampled 3-4 years, what magnitudes of trend were we likely to detect for various species?

2) Would varying the number of sites, the number of transects, or the number of samples per transect result in large differences to statistical power?

3) Is statistical power more sensitive to changes in sampling design (e.g., number of sites, \# of transects, \# samples/transect, etc.) or to changes in parameters (e.g., different levels of alpha)?

4) Would varying the sampling type (point sampling of quadrats, horizontal transects or vertical transects) result in large differences to statistical power? If there were differences in power among the sampling types, is that difference related to the number of points sampled in each?

The analysis run for Glacier Bay (coarse-grained sites) was a post-hoc analysis, in that the power estimates were based on detecting trends within the time period already sampled. With 3-4 years of data, linear regression was run on untransformed percent cover estimates for each transect. The variance of the residuals from the linear regression was input to MONITOR as the site variance for each site. The average cover over all transects over all sampled years was input as the site mean for each site. The effects of different sampling designs were analyzed by estimating different variance estimates corresponding to each sampling design.

\section{Issues with MONITOR (as used)}

For exponential trend, a log-normal distribution for within-year sampling variability at each site was assumed. This assumption was not tested to see if it was a reasonable one for the Glacier Bay cover data.

The new version of MONITOR allows the sampling variance to be partitioned between within-year and between-year components. The authors do not expect this to be a critical difference to the power results, but it would be interesting to recalculate power using this feature. The variance components could be estimated from existing data.

MONITOR 10.0 also allows the user to specify if the mean and the variance are related over time. This is a very common scenario, and changes to this component may affect the power estimates more than any of the other issues mentioned here.

Issues with Data Analysis and Input Parameters

Although this does not affect the power analysis, I am not sure why there is not an analysis of the observed trends in the report. Since the researchers expect an exponential trend, and this is a post-hoc power analysis, the analysis itself should have been conducted first. In the process of selecting the best analysis, the distribution of the errors would be tested and the shapes of the trends could be discussed. This would also alert the analyst as to whether there are any errors in the data set.

The plot or site means input into program MONITOR should have been the starting mean values in Year 0 , rather than the average across all years. If the research question were to detect trends in an ongoing monitoring program, the mean of all current data might be appropriate, but in the case of a post-hoc test such as this, the mean of the first year of sampling should be used. This is not likely to have a large effect on the power results. 
The sampling variance should be estimated by the empirical variance of the residuals from an exponential regression fit, rather than a linear fit. This would match the assumptions of program MONITOR. The results are likely to be similar, depending on the magnitude and shape of any existing trends.

There are some other minor things that I may have done differently, but as stated in the start of this document, I think the overall methods were reasonable.

\section{Limited Re-analysis}

To answer some of the issues raised above, I evaluated data for MY, FU, and BARN collected in 19982001 on six vertical transects with five points per meter. Results reported below include an analysis of existing trends, data distributions, revised parameter estimates, and limited statistical power results from MONITOR 10.0 for comparison purposes. The intention is to determine if the changes to program MONITOR and/or changes to parameters suggested above cause large differences in estimated statistical power.

\section{Testing Assumptions}

The metric being tested for trends at this site is percent cover, estimated by counts divided by the number of points sampled (multiplied by 100). The estimates can exceed $100 \%$ due to layered species.

\section{MY}

I subtracted out the site/year means and tested the combined residuals for normality using Shapiro-Wilks goodness-of-fit test with alpha $=0.05$. The tails of the distribution were too thin to fit a normal distribution ( $p$ value $=0.0000$ ), but there was no skew. When the sites were tested separately, all but three had approximately normal residuals (sites 63 and 151 have a slight skew, and site 220 appears bimodal). This could indicate that variance differs across sites or that these three sites were causing the lack of normality overall. When the residuals were standardized for variance, the distribution was skewed with a shortened range $(p$-value $=0.0000)$.

I also log-transformed the cover (after adding the constant 1 to allow for zeros) and tested the combined residuals for normality with the same result (no improvement in fit for log-normal assumption). All but three individual sites (site 36 and 215 had a few "outlier" transects with low cover; site 220 appears bimodal) were approximately normal after log-transformation.

The above indicates that neither normal nor log-normal simulations are ideal for estimating statistical power for these data. An empirical distribution or a box-cox transformation would provide a better fit. However, MONITOR 10.0 is the best available software for estimating the power.

The mean (site/year) and variance are related, as shown in figure B1, so the constant CV option in MONITOR 10.0 should be selected.

\section{FU}

The distribution of the combined residuals again had thin tails and did not fit a normal distribution ( $p$-value $=$ 0.0000 ). When the sites were tested separately, nine sites failed the normality test - mainly for skewness. The log-transformed combined residuals are approximately normally distributed (Shapiro-Wilks $p$-value $=$ 0.19). The lognormal distribution is the appropriate choice for FU. As with MY, the mean and variance are related (see fig. B2). 
BARN

The distribution of the combined residuals again had thin tails and did not fit a normal distribution ( $\mathrm{p}$-value $=$ $0.0001)$. When the sites were tested separately, four sites failed the normality test - mainly for skewness (sites $63,108,200$, and 218 ). The log-transformation did not approximate normality ( $p$-value $=0.0001$ ), with four different individual sites also failing (sites $69,163,214$, and 216 ). As with the other species, the mean and variance are related (see fig. B3).

\section{Testing for Exponential Trends}

A linear regression was fit to log-transformed density data for each site for each species to test for exponential trends.

\section{MY}

The trends (slopes) for MY ranged from a decrease of $50 \%$ per year (Willoughby Island - West) to an increase of $125 \%$ per year (Muir Inlet - Upper), and the slopes were approximately normally distributed. The average trend was a decrease of $8 \%$, which was not significantly different from zero using a two-tailed $\mathrm{t}$-test $(\mathrm{p}$-value $=0.21$ ). Trends for individual sites are displayed in table B1. There are significant positive and negative trends observed at some individual sites, but no significant regional trend in MY over the years 1998-2001. Note that the individual regression significance results are only valid if the measurements from year to year are independent. Since the transects are randomly selected in each year, these should be approximately independent.

\section{FU}

The trends for FU ranged from a decrease of 70\% per year (Russel Island) to an increase of $220 \%$ per year (Berg Bay), and the slopes were approximately normally distributed. The average trend was an increase of $13 \%$, which was not significantly different from zero using a two-tailed t-test ( $p$-value $=0.27$ ). Trends for individual sites are displayed in table B2. There are significant positive and negative trends observed at some individual sites, but no significant regional trend in FU over the years 1998-2001.

\section{BARN}

The trends for BARN ranged from a decrease of $16 \%$ per year (Muir Inlet - Lower) to an increase of $77 \%$ per year (Lester Island). The distribution of slopes failed the test for normality at alpha $=0.05$ ( $p$-value $=$ $0.0095)$. However, the t-test is fairly robust to non-normality, and the result for the nonparametric Wilcoxon test was similar. The average trend was an increase of $22.5 \%$, which was significantly different from zero using a two-tailed t-test $(p$-value $=0.00005)$. Trends for individual sites are displayed in table B3. There are significant positive trends observed at some individual sites, and a significant increasing regional trend in BARN over the years 1998-2001.

\section{MONITOR Parameter Estimates}

The plot means for MONITOR are the starting means for the trends. These should be the 1998 plot means, as displayed in tables B4-6. The plot variances, shown in the tables as standard deviations, were estimated by the residual variance from exponential regressions fit to untransformed densities. These plot variances include all sources of variability: spatial variability, sampling error, and random variability due to year effects (e.g., weather). The final version of MONITOR 10.0 will have capability to account for random variability due to year as a separate parameter, but in the existing beta version (as of 5/7/07) this capability is not correctly working (personal communication, James Gibbs). However, the parameters may be of some interest in understanding the sources of variability for the different species. The variance components for 
between versus within years (displayed in tables B4-6 as standard deviations) were estimated using restricted maximum likelihood estimates for random components of variance model.

For MY, the variation among years (after adjusting for trend) is effectively zero for all but six sites. At least for the existing three years of data, the majority of sites have very little variance due to random year effects (relative to within year variance). In this case, partitioning the variance is unlikely to have a strong effect on statistical power. The two plots in figure B4 show an example of two sites with different variance characteristics. MY cover at Shag Cove, in the top plot, has very little variability among years after the trend is removed. At Upper Drake Island, however, there is variability both among and within years.

For FU, the results are similar to those for MY, except that there are nine sites with some annual variability.

Barnacles have mixed results across sites, with some sites exhibiting more variability among years than within years. Thus, power for BARN would be the most likely to change if the components of variance are split.

\section{MONITOR 10.0 Analysis}

The statistical power was estimated using MONITOR 10.0 dated 3/14/07 at this web address:

\section{http://www.esf.edu/efb/gibbs/MONITOR/.}

Power was estimated with the original parameters used by USGS, as well as with the new parameters estimated and discussed above. The plot means were originally the mean of all samples across three years, whereas I used the means of the first year's samples only. The plot standard deviations were originally the standard error of the residuals from a linear regression on untransformed data. I used the standard error of the residuals from an exponential regression. For reduced numbers of sites, the original analysis used one random subset of sites to estimate the power. I averaged the statistical power over 10 random subsets of the 25 sites. I also used 1000 instead of 500 simulations in MONITOR, to create more stable results.

The plots in figures B5 and B6 show the power estimates for MY with 25 and 15 sites with 6 transects, each having five points per meter. The yellow triangles show results from the original report (partial series). The brown circles show the current runs on MONITOR 10.0 using the original parameters, but with 1000 replications. The blue squares show the current runs on MONITOR 10.0 with the TerraStat parameters. The results show that the original power estimates for MY using earlier parameter estimates and previous versions of MONITOR were almost identical to current estimates.

The plot in figure B7 shows that the change in parameterization had the effect of reducing the statistical power for FU by $5-10 \%$. This is likely due to the reduced plot mean estimates - the new parameters represent an average decrease of $4 \%$ in the starting mean. In contrast, Mytilus and Barnacle plot means had an average increase in starting mean (although there was high variability across sites.) Results for FU using MONITOR 10 are similar to previous results when the original parameters are used. The power for 15 sites was not estimated in the previous report.

The plot in figure B8 shows that the original power results for BARN are equivalent to the estimates with MONITOR 10, and with revised parameters. 


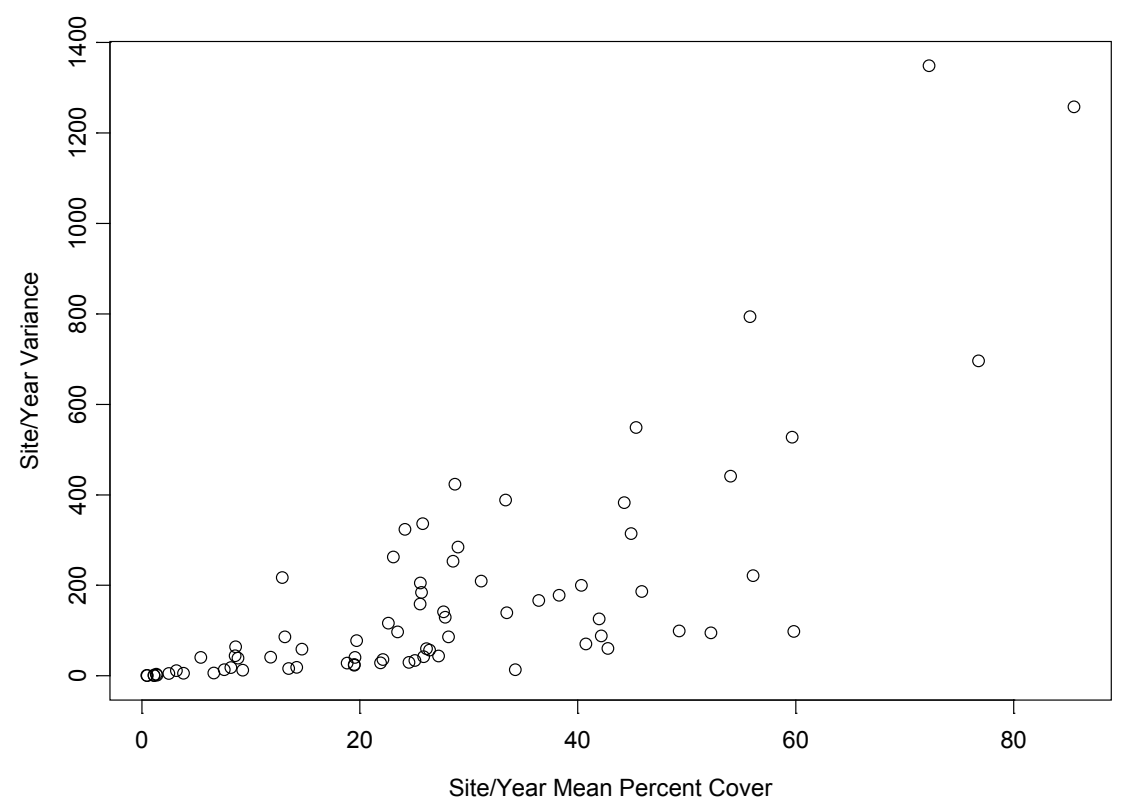

Figure B1. Variance of MY cover within a year at each site plotted against the average cover.

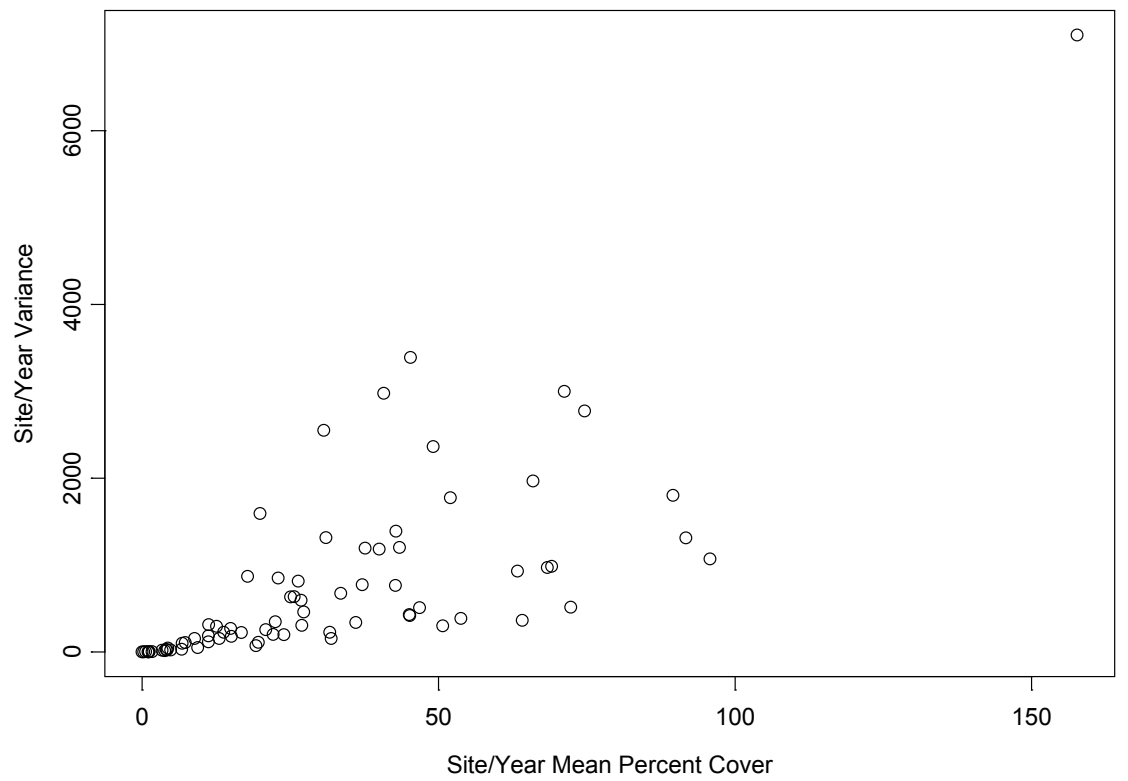

Figure B2. Variance of FU cover within a year at each site plotted against the average cover. 


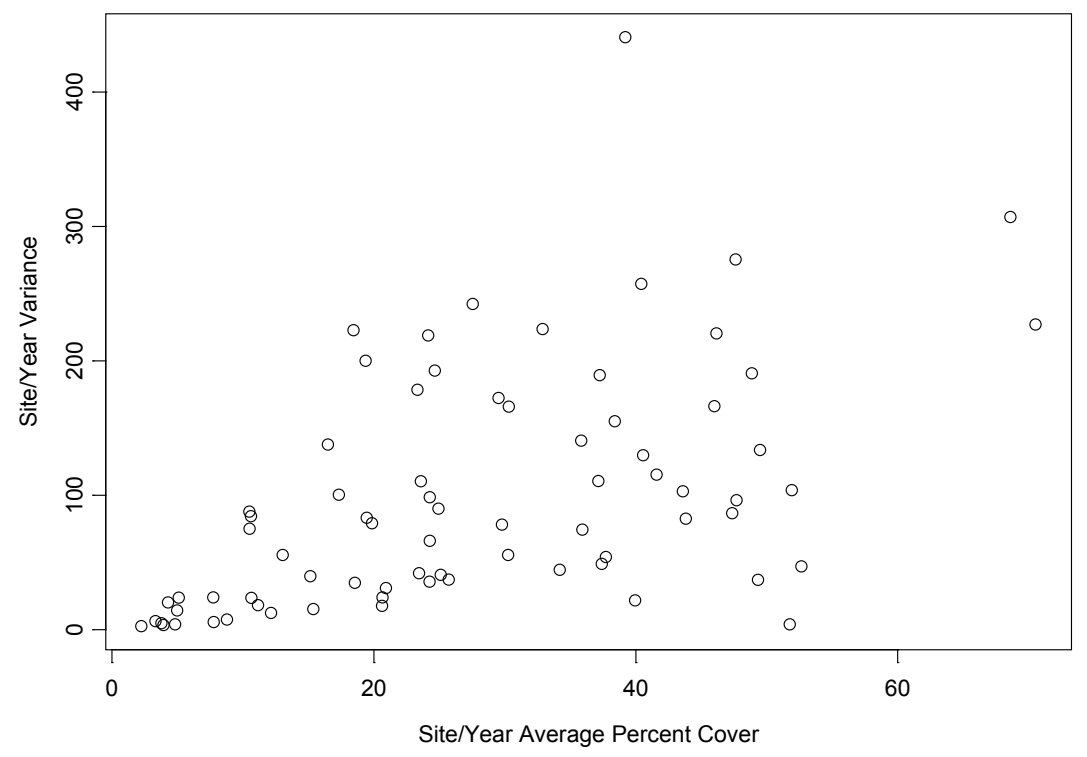

Figure B3. Variance of BARN cover within a year at each site plotted against the average cover.

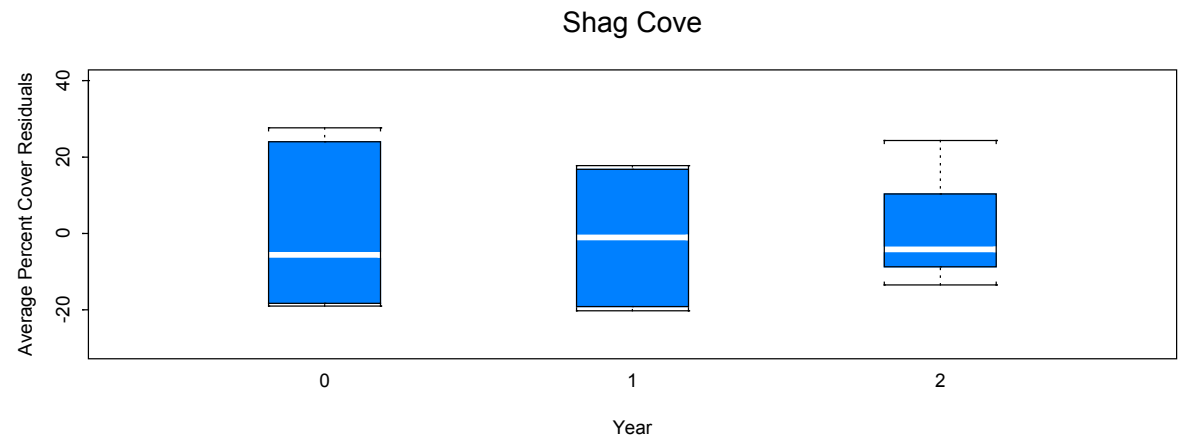

Drake Island, Upper

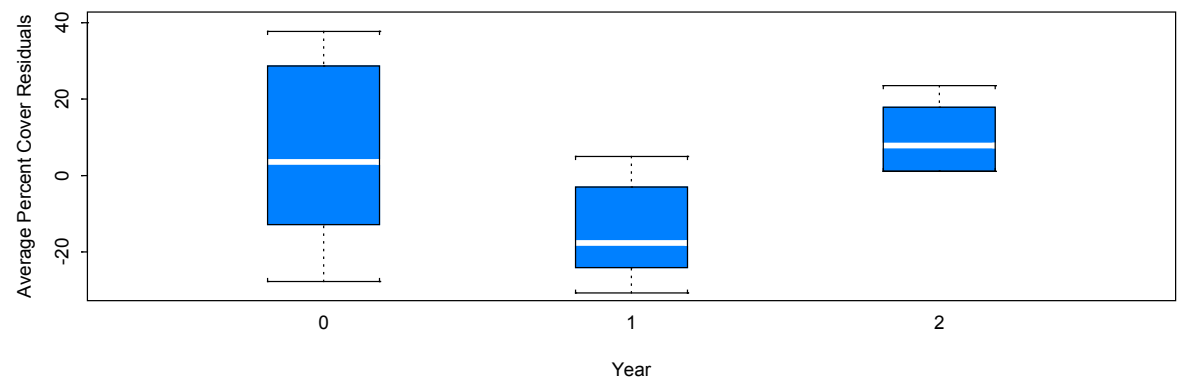

FigureB4. Comparison of residual variance sources within and between years for MY percent cover for two selected sites. 


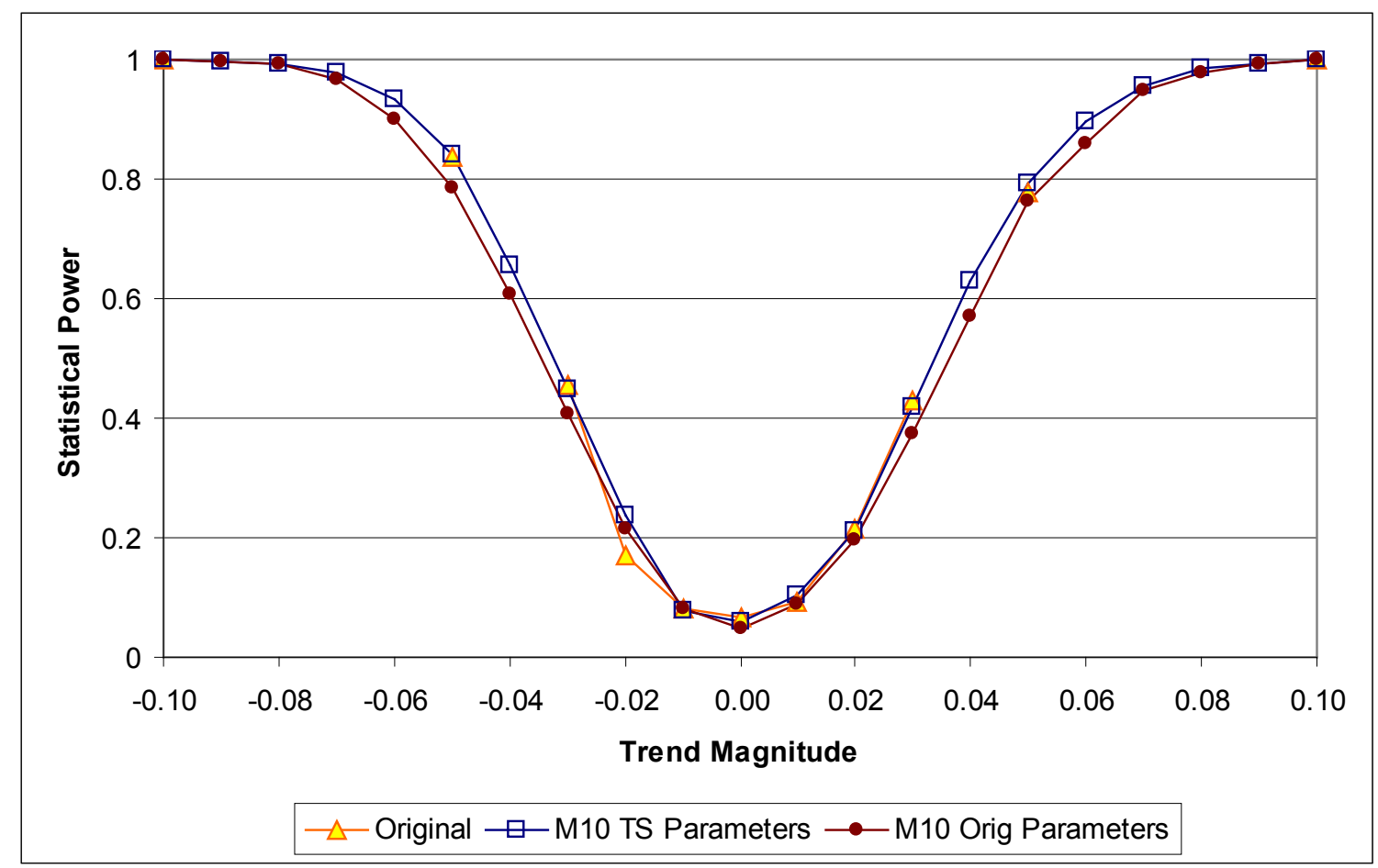

Figure B5. Statistical power comparison for MY with 25 sites.

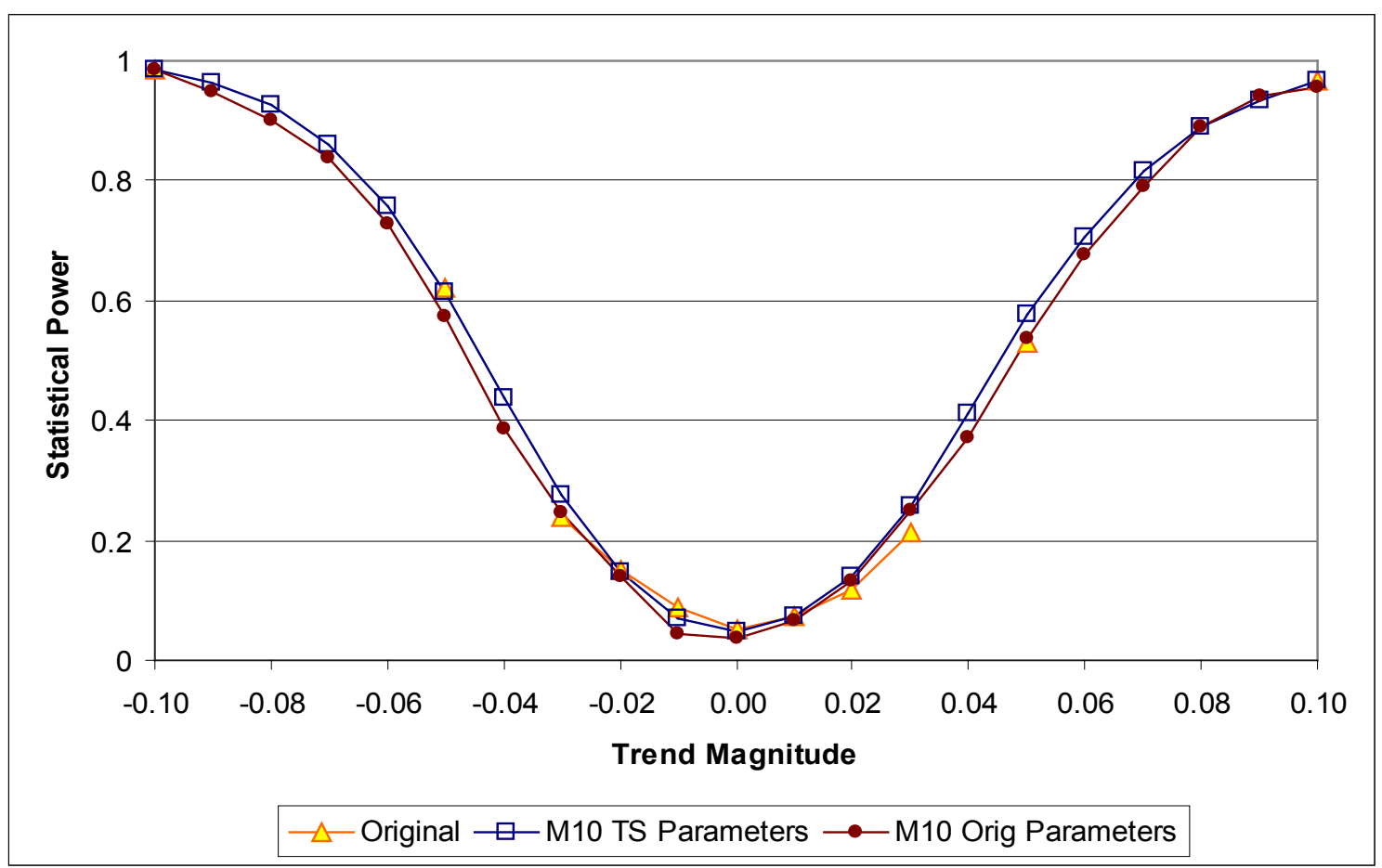

Figure B6. Statistical power comparison for MY with 15 sites. 


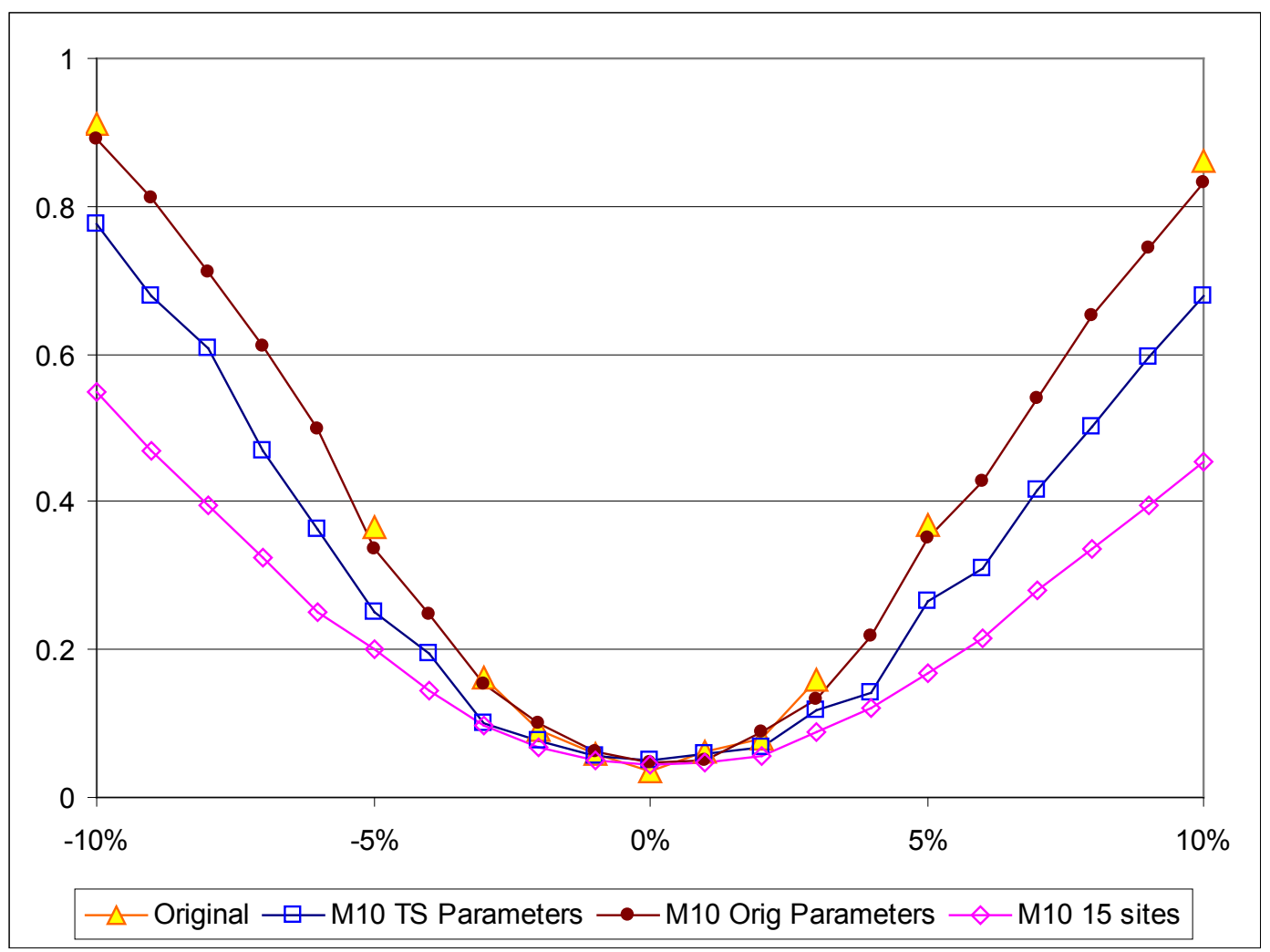

Figure B7. Statistical power comparison for FU.

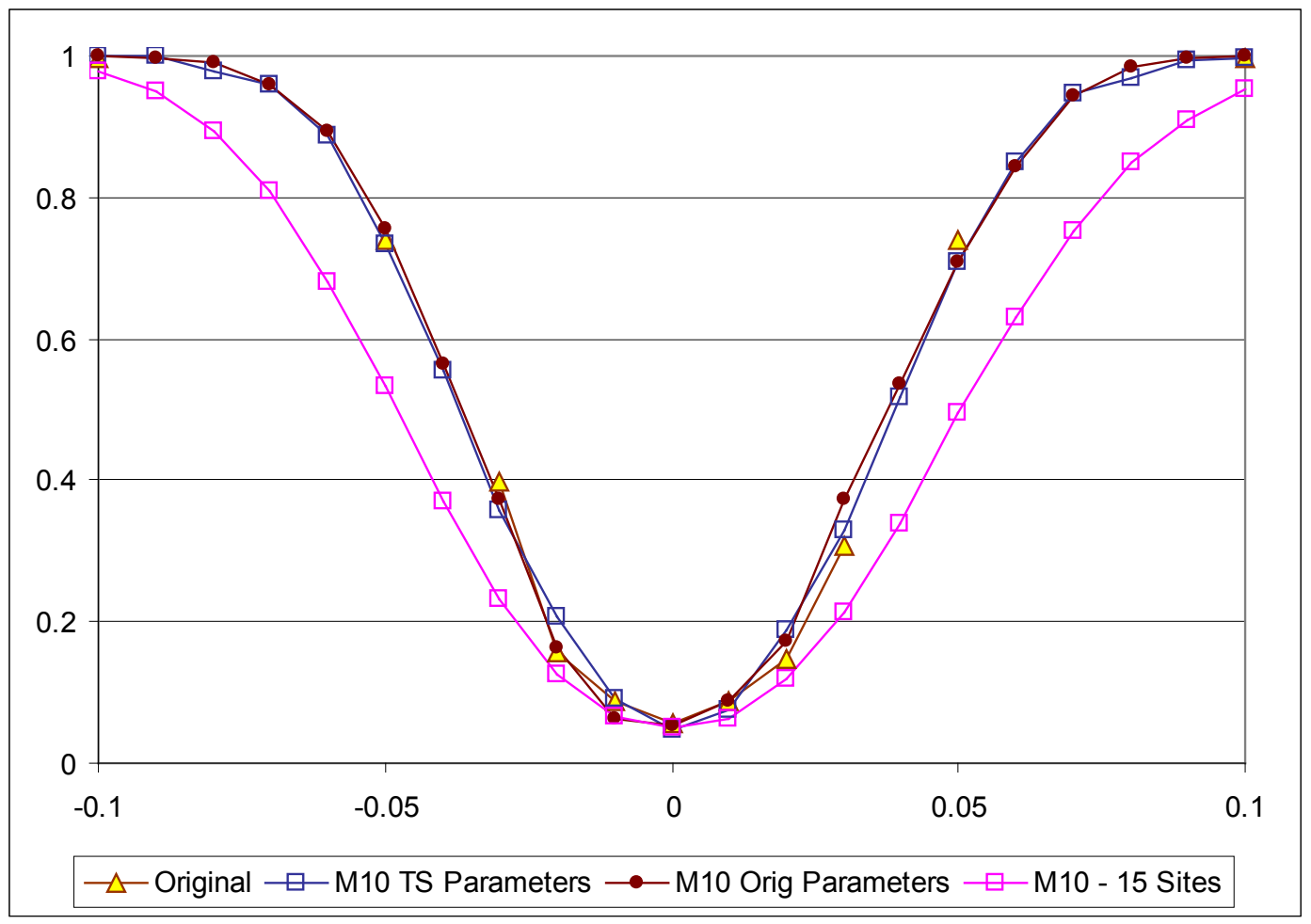

Figure B8. Statistical power comparison for BARN. 
Table B1. Trend results by site for percent cover of MY.

\begin{tabular}{|clccc|}
\hline $\begin{array}{c}\text { Site } \\
\text { Number }\end{array}$ & Site Name & Slope & p-value & Trend \\
\hline 36 & Bear Track & 0.126 & 0.472 & \\
69 & Berg Bay & 0.288 & 0.023 & + \\
63 & Between Pt Carolus \& Ripple Cove & -0.385 & 0.109 & \\
113 & Blue Mouse Cove & 0.076 & 0.273 & \\
217 & Drake Island - Lower & -0.107 & 0.111 & \\
216 & Drake Island - Mid & -0.434 & 0.001 & - \\
218 & Drake Island - Upper & -0.200 & 0.089 & \\
89 & Geikie Inlet - Lower & 0.036 & 0.747 & \\
88 & Geikie Inlet - Upper & 0.154 & 0.150 & \\
59 & Lester Island & -0.218 & 0.209 & \\
215 & Little Sturgess Island & -0.059 & 0.803 & \\
143 & Mt Abdallah Outwash & -0.112 & 0.242 & \\
12 & Muir Inlet - Lower & 0.405 & 0.002 & + \\
2 & Muir Inlet - Upper & 0.813 & 0.000 & + \\
62 & Pt Carolus & -0.167 & 0.100 & \\
151 & Rendu Inlet & 0.102 & 0.763 & \\
200 & Russel Island & -0.471 & 0.010 & - \\
108 & Scidmore Bay & -0.024 & 0.886 & \\
220 & Shag Cove & -0.135 & 0.212 & \\
214 & Sturgess Island & -0.224 & 0.177 & \\
142 & Tarr Inlet & -0.014 & 0.867 & \\
164 & Tidal Inlet - Lower & -0.071 & 0.576 & \\
163 & Tidal Inlet - Upper & -0.389 & 0.004 & - \\
223 & Willoughby Island - East & -0.282 & 0.029 & - \\
224 & Willoughby Island - West & -0.692 & 0.001 & - \\
& & & & \\
\hline
\end{tabular}


Table B2. Trend results by site for percent cover of FU.

\begin{tabular}{|c|c|c|c|c|}
\hline $\begin{array}{c}\text { Site } \\
\text { Number }\end{array}$ & Site & Slope & p-value & Trend \\
\hline 36 & Bear Track & -0.611 & 0.055 & \\
\hline 69 & Berg Bay & 1.17 & 0.000 & + \\
\hline 63 & Between Pt Carolus \& Ripple Cove & 0.641 & 0.111 & \\
\hline 113 & Blue Mouse Cove & 0.068 & 0.908 & \\
\hline 217 & Drake Island - Lower & -1.01 & 0.002 & - \\
\hline 216 & Drake Island - Mid & 0.411 & 0.047 & + \\
\hline 218 & Drake Island - Upper & 0.157 & 0.339 & \\
\hline 89 & Geikie Inlet - Lower & 0.818 & 0.001 & + \\
\hline 88 & Geikie Inlet - Upper & 0.555 & 0.015 & + \\
\hline 59 & Lester Island & 0.444 & 0.211 & \\
\hline 215 & Little Sturgess Island & 0.410 & 0.407 & \\
\hline 143 & Mt Abdallah Outwash & -0.120 & 0.564 & \\
\hline 12 & Muir Inlet - Lower & 0.152 & 0.652 & \\
\hline 2 & Muir Inlet - Upper & 0.253 & 0.451 & \\
\hline 62 & Pt Carolus & 0.317 & 0.097 & \\
\hline 151 & Rendu Inlet & 0.310 & 0.471 & \\
\hline 200 & Russel Island & -1.212 & 0.006 & - \\
\hline 108 & Scidmore Bay & 0.103 & 0.743 & \\
\hline 220 & Shag Cove & 0.107 & 0.660 & \\
\hline 214 & Sturgess Island & 0.217 & 0.510 & \\
\hline 142 & Tarr Inlet & -0.352 & 0.267 & \\
\hline 164 & Tidal Inlet - Lower & 0.421 & 0.055 & \\
\hline 163 & Tidal Inlet - Upper & -0.653 & 0.088 & \\
\hline 223 & Willoughby Island - East & -0.149 & 0.655 & \\
\hline 224 & Willoughby Island - West & 0.665 & 0.064 & \\
\hline
\end{tabular}


Table B3. Trend results by site for percent cover of BARN.

\begin{tabular}{|clccc|}
\hline $\begin{array}{c}\text { Site } \\
\text { Number }\end{array}$ & Site & Slope & p-value & Trend \\
\hline 36 & Bear Track & -0.011 & 0.974 & \\
69 & Berg Bay & 0.071 & 0.771 & \\
63 & Between Pt Carolus \& Ripple Cove & 0.116 & 0.713 & \\
113 & Blue Mouse Cove & 0.449 & 0.019 & + \\
217 & Drake Island - Lower & 0.002 & 0.989 & \\
216 & Drake Island - Mid & 0.097 & 0.557 & \\
218 & Drake Island - Upper & 0.093 & 0.676 & \\
89 & Geikie Inlet - Lower & -0.014 & 0.887 & \\
88 & Geikie Inlet - Upper & 0.279 & 0.333 & \\
59 & Lester Island & 0.570 & 0.057 & \\
215 & Little Sturgess Island & 0.437 & 0.026 & + \\
143 & Mt Abdallah Outwash & 0.373 & 0.004 & + \\
12 & Muir Inlet - Lower & -0.169 & 0.076 & \\
2 & Muir Inlet - Upper & 0.494 & 0.018 & + \\
62 & Pt Carolus & -0.039 & 0.899 & \\
151 & Rendu Inlet & 0.462 & 0.092 & \\
200 & Russel Island & 0.426 & 0.013 & + \\
108 & Scidmore Bay & 0.468 & 0.083 & \\
220 & Shag Cove & 0.044 & 0.668 & \\
214 & Sturgess Island & 0.007 & 0.979 & \\
142 & Tarr Inlet & 0.363 & 0.259 & \\
164 & Tidal Inlet - Lower & 0.008 & 0.962 & \\
163 & Tidal Inlet - Upper & 0.049 & 0.795 & \\
223 & Willoughby Island - East & 0.470 & 0.006 & + \\
224 & Willoughby Island - West & 0.350 & 0.022 & + \\
& & & & \\
\hline
\end{tabular}


Table B4. Plot means and standard deviations for MY percent cover.

\begin{tabular}{|c|c|c|c|c|c|c|c|c|c|}
\hline $\begin{array}{c}\text { Site } \\
\text { Number }\end{array}$ & Site & 1998 & lot Me & 2001 & $\begin{array}{c}\text { USGS } \\
\text { Original } \\
\text { Mean } \\
\text { Estimate }\end{array}$ & $\begin{array}{c}\text { Plot } \\
\text { Standard } \\
\text { Deviation }\end{array}$ & $\begin{array}{c}\text { USGS } \\
\text { Original } \\
\text { SD } \\
\text { Estimate }\end{array}$ & $\begin{array}{c}\text { Standard } \\
\text { Deviation } \\
\text { Among } \\
\text { Years }\end{array}$ & $\begin{array}{c}\text { Standard } \\
\text { Deviation } \\
\text { Within } \\
\text { Years }\end{array}$ \\
\hline 2 & Muir Inlet - Upper & 2.48 & 4.59 & 14.2 & 6.8 & 2.99 & 3.26 & 0.00 & 2.99 \\
\hline 12 & Muir Inlet - Lower & 19.7 & 14.7 & 42.8 & 27.4 & 8.42 & 8.45 & 4.04 & 7.70 \\
\hline 36 & Bear Track & 11.8 & 7.6 & 13.5 & 11.0 & 5.15 & 5.33 & 2.14 & 4.83 \\
\hline 59 & Lester Island & 1.39 & 1.34 & 0.47 & 1.07 & 1.26 & 1.28 & 0.00 & 1.26 \\
\hline 62 & Pt Carolus & 1.13 & 1.12 & 0.520 & 0.9 & 0.679 & 0.686 & 0.00 & 0.679 \\
\hline 63 & Between Pt Carolus \& Ripple Cove & 8.57 & 5.41 & 3.19 & 5.73 & 5.30 & 5.50 & 0.00 & 5.30 \\
\hline 69 & Berg Bay & 25.6 & 33.5 & 42.0 & 33.7 & 11.8 & 12.2 & 0.00 & 11.8 \\
\hline 88 & Geikie Inlet - Upper & 6.61 & 8.18 & 9.27 & 8.02 & 3.27 & 3.38 & 0.00 & 3.27 \\
\hline 89 & Geikie Inlet - Lower & 28.2 & 27.7 & 31.1 & 29.0 & 11.4 & 11.7 & 0.00 & 11.4 \\
\hline 108 & Scidmore Bay & 28.7 & 28.6 & 25.7 & 27.7 & 15.9 & 16.4 & 0.00 & 15.9 \\
\hline 113 & Blue Mouse Cove & 22.1 & 21.9 & 25.9 & 23.3 & 5.67 & 5.80 & 0.00 & 5.67 \\
\hline 142 & Tarr Inlet & 26.1 & 18.8 & 25.1 & 23.3 & 6.81 & 7.03 & 2.90 & 6.36 \\
\hline 143 & Mt Abdallah Outwash & 24.5 & 23.5 & 19.5 & 22.5 & 6.69 & 6.85 & 0.00 & 6.69 \\
\hline 151 & Rendu Inlet & 8.61 & 12.9 & 8.8 & 10.1 & 9.90 & 10.2 & 0.00 & 9.90 \\
\hline 163 & Tidal Inlet - Upper & 26.4 & 19.5 & 13.1 & 19.7 & 7.02 & 7.34 & 0.00 & 7.02 \\
\hline 164 & Tidal Inlet - Lower & 59.7 & 59.8 & 55.8 & 58.4 & 20.5 & 21.1 & 0.00 & 20.5 \\
\hline 200 & Russel Island & 56.1 & 45.4 & 25.8 & 42.2 & 17.9 & 18.2 & 0.00 & 17.9 \\
\hline 214 & Sturgess Island & 22.6 & 27.8 & 14.7 & 21.7 & 10.5 & 10.5 & 3.62 & 10.1 \\
\hline 215 & Little Sturgess Island & 33.4 & 29.0 & 23.1 & 28.5 & 16.6 & 17.1 & 0.00 & 16.6 \\
\hline 216 & Drake Island - Mid & 72.2 & 45.9 & 27.2 & 48.4 & 21.6 & 22.8 & 0.00 & 21.6 \\
\hline 217 & Drake Island - Lower & 52.2 & 34.3 & 42.2 & 42.9 & 9.7 & 10.6 & 6.48 & 8.08 \\
\hline 218 & Drake Island - Upper & 76.8 & 38.3 & 49.3 & 54.8 & 20.2 & 22.5 & 10.9 & 18.0 \\
\hline 220 & Shag Cove & 54.0 & 44.9 & 40.3 & 46.4 & 16.8 & 17.4 & 0.00 & 16.8 \\
\hline 223 & Willoughby Island - East & 40.8 & 36.4 & 25.5 & 34.2 & 11.0 & 11.1 & 0.00 & 11.0 \\
\hline 224 & Willoughby Island - West & 85.5 & 44.3 & 24.2 & 51.3 & 24.0 & 26.7 & 0.00 & 24.0 \\
\hline
\end{tabular}


Table B5. Plot means and standard deviations for FU percent cover.

\begin{tabular}{|c|c|c|c|c|c|c|c|c|c|}
\hline $\begin{array}{c}\text { Site } \\
\text { Number }\end{array}$ & Site & 1998 & Jot Mea & 2001 & $\begin{array}{c}\text { USGS } \\
\text { Original } \\
\text { Mean } \\
\text { Estimate }\end{array}$ & $\begin{array}{c}\text { Plot } \\
\text { Standard } \\
\text { Deviation }\end{array}$ & $\begin{array}{c}\text { USGS } \\
\text { Original } \\
\text { SD } \\
\text { Estimate }\end{array}$ & $\begin{array}{c}\text { Standard } \\
\text { Deviation } \\
\text { Among } \\
\text { Years }\end{array}$ & $\begin{array}{c}\text { Standard } \\
\text { Deviation } \\
\text { Within } \\
\text { Years }\end{array}$ \\
\hline 2 & Muir Inlet - Upper & 25.6 & 49.1 & 33.5 & 36.1 & 34.2 & 35.4 & 0.00 & 34.2 \\
\hline 12 & Muir Inlet - Lower & 3.37 & 19.2 & 4.85 & 9.14 & 9.34 & 9.62 & 8.36 & 6.15 \\
\hline 36 & Bear Track & 12.6 & 1.22 & 1.13 & 4.97 & 9.41 & 10.4 & 0.00 & 9.41 \\
\hline 59 & Lester Island & 14.9 & 22.5 & 26.9 & 21.4 & 16.5 & 17.1 & 0.00 & 16.5 \\
\hline 62 & Pt Carolus & 31.7 & 53.7 & 50.7 & 45.4 & 17.6 & 18.5 & 3.15 & 17.4 \\
\hline 63 & Between Pt Carolus \& Ripple Cove & 4.08 & 19.9 & 22.9 & 15.6 & 27.3 & 28.2 & 0.00 & 27.3 \\
\hline 69 & Berg Bay & 1.67 & 4.21 & 23.9 & 9.94 & 8.14 & 8.75 & 0.00 & 8.14 \\
\hline 88 & Geikie Inlet - Upper & 0 & 0.282 & 4.34 & 1.54 & 3.66 & 3.82 & 0.00 & 3.7 \\
\hline 89 & Geikie Inlet - Lower & 9.37 & 26.8 & 46.8 & 27.7 & 18.6 & 19.1 & 0.00 & 18.6 \\
\hline 108 & Scidmore Bay & 19.6 & 36.1 & 37.6 & 31.1 & 22.4 & 23.2 & 0.00 & 22.4 \\
\hline 113 & Blue Mouse Cove & 45.3 & 40.8 & 26.3 & 37.5 & 46.1 & 47.4 & 0.00 & 46.1 \\
\hline 142 & Tarr Inlet & 3.80 & 13.0 & 1.00 & 5.94 & 8.76 & 8.81 & 5.38 & 7.5 \\
\hline 143 & Mt Abdallah Outwash & 45.2 & 91.7 & 40.0 & 59.0 & 37.8 & 38.3 & 25.4 & 31.2 \\
\hline 151 & Rendu Inlet & 7.30 & 11.2 & 13.8 & 10.8 & 13.8 & 14.2 & 0.00 & 13.8 \\
\hline 163 & Tidal Inlet - Upper & 8.84 & 11.2 & 0.581 & 6.86 & 9.64 & 9.54 & 1.75 & 9.53 \\
\hline 164 & Tidal Inlet - Lower & 43.4 & 74.7 & 72.3 & 63.5 & 37.4 & 38.9 & 0.00 & 37.4 \\
\hline 200 & Russel Island & 63.3 & 37.1 & 11.2 & 37.2 & 23.6 & 24.4 & 0.00 & 23.6 \\
\hline 214 & Sturgess Island & 71.2 & 22.1 & 68.4 & 53.9 & 42.0 & 43.2 & 22.9 & 37.3 \\
\hline 215 & Little Sturgess Island & 17.8 & 52.0 & 31.0 & 33.6 & 36.7 & 38.1 & 6.58 & 36.3 \\
\hline 216 & Drake Island - Mid & 42.8 & 42.8 & 89.5 & 58.4 & 35.2 & 36.0 & 0.00 & 35.2 \\
\hline 217 & Drake Island - Lower & 95.8 & 64.1 & 25.1 & 61.6 & 25.5 & 25.7 & 0.00 & 25.5 \\
\hline 218 & Drake Island - Upper & 31.9 & 27.3 & 45.0 & 34.7 & 18.2 & 18.6 & 0.00 & 18.2 \\
\hline 220 & Shag Cove & 65.9 & 158 & 69.1 & 97.6 & 69.8 & 71.6 & 46.4 & 57.9 \\
\hline 223 & Willoughby Island - East & 16.8 & 6.70 & 15.1 & 12.8 & 12.1 & 12.5 & 2.02 & 12.0 \\
\hline 224 & Willoughby Island - West & 6.74 & 20.9 & 30.6 & 19.4 & 29.4 & 30.3 & 0.00 & 29.4 \\
\hline
\end{tabular}


Table B6. Plot means and standard deviations for BARN percent cover.

\begin{tabular}{|c|c|c|c|c|c|c|c|c|c|}
\hline $\begin{array}{c}\text { Site } \\
\text { Number }\end{array}$ & Site & 1998 & ot Mea & 2001 & $\begin{array}{c}\text { USGS } \\
\text { Original } \\
\text { Mean } \\
\text { Estimate }\end{array}$ & $\begin{array}{c}\text { Plot } \\
\text { Standard } \\
\text { Deviation }\end{array}$ & $\begin{array}{c}\text { USGS } \\
\text { Original } \\
\text { SD } \\
\text { Estimate }\end{array}$ & $\begin{array}{c}\text { Standard } \\
\text { Deviation } \\
\text { Among } \\
\text { Years }\end{array}$ & $\begin{array}{c}\text { Standard } \\
\text { Deviation } \\
\text { Within } \\
\text { Years } \\
\end{array}$ \\
\hline 2 & Muir Inlet - Upper & 3.79 & 4.99 & 8.79 & 5.86 & 2.81 & 2.88 & 0.0 & 2.8 \\
\hline 12 & Muir Inlet - Lower & 52.7 & 37.7 & 40.4 & 43.6 & 11.0 & 11.8 & 1.8 & 10.9 \\
\hline 36 & Bear Track & 24.3 & 2.25 & 24.3 & 16.9 & 12.0 & 12.2 & 12.5 & 5.9 \\
\hline 59 & Lester Island & 7.77 & 3.32 & 24.9 & 12.0 & 6.73 & 7.44 & 4.0 & 5.8 \\
\hline 62 & Pt Carolus & 25.1 & 3.94 & 24.3 & 17.8 & 12.0 & 12.2 & 11.6 & 6.9 \\
\hline 63 & Between Pt Carolus \& Ripple Cove & 16.5 & 5.11 & 19.4 & 13.7 & 12.0 & 12.3 & 5.8 & 11.0 \\
\hline 69 & Berg Bay & 41.6 & 13.1 & 47.4 & 34.0 & 17.5 & 17.5 & 17.6 & 9.3 \\
\hline 88 & Geikie Inlet - Upper & 10.6 & 4.30 & 15.4 & 10.1 & 7.17 & 7.27 & 4.0 & 6.3 \\
\hline 89 & Geikie Inlet - Lower & 37.2 & 29.8 & 35.8 & 34.3 & 11.4 & 11.8 & 0.0 & 11.4 \\
\hline 108 & Scidmore Bay & 18.5 & 10.5 & 39.2 & 22.7 & 16.6 & 17.0 & 5.8 & 15.8 \\
\hline 113 & Blue Mouse Cove & 30.3 & 19.9 & 68.6 & 39.6 & 17.1 & 17.3 & 12.3 & 13.6 \\
\hline 142 & Tarr Inlet & 25.7 & 4.84 & 51.8 & 27.4 & 14.1 & 15.0 & 16.2 & 3.9 \\
\hline 143 & Mt Abdallah Outwash & 20.9 & 18.6 & 43.8 & 27.8 & 8.49 & 8.30 & 5.7 & 7.0 \\
\hline 151 & Rendu Inlet & 17.3 & 7.74 & 37.2 & 20.7 & 11.4 & 11.6 & 8.5 & 8.8 \\
\hline 163 & Tidal Inlet - Upper & 29.5 & 12.2 & 32.9 & 24.9 & 14.3 & 14.5 & 9.9 & 11.7 \\
\hline 164 & Tidal Inlet - Lower & 46.0 & 24.7 & 47.6 & 39.4 & 17.4 & 17.7 & 11.3 & 14.5 \\
\hline 200 & Russel Island & 30.3 & 24.2 & 70.5 & 42.7 & 15.3 & 15.3 & 10.2 & 12.8 \\
\hline 214 & Sturgess Island & 40.6 & 10.5 & 39.9 & 30.3 & 16.6 & 16.9 & 16.8 & 8.7 \\
\hline 215 & Little Sturgess Island & 20.7 & 11.2 & 49.5 & 27.1 & 11.5 & 12.1 & 10.2 & 7.7 \\
\hline 216 & Drake Island - Mid & 35.9 & 15.2 & 43.6 & 31.6 & 14.2 & 14.2 & 13.6 & 8.5 \\
\hline 217 & Drake Island - Lower & 47.7 & 20.6 & 48.9 & 39.1 & 16.4 & 16.7 & 15.4 & 10.1 \\
\hline 218 & Drake Island - Upper & 37.4 & 10.7 & 46.2 & 31.4 & 17.5 & 17.5 & 17.2 & 9.9 \\
\hline 220 & Shag Cove & 34.2 & 23.5 & 38.4 & 32.0 & 10.5 & 10.6 & 6.4 & 9.0 \\
\hline 223 & Willoughby Island - East & 23.3 & 19.5 & 51.9 & 31.6 & 12.4 & 12.7 & 6.8 & 11.0 \\
\hline 224 & Willoughby Island - West & 27.6 & 23.6 & 49.3 & 33.5 & 12.4 & 12.4 & 5.7 & 11.4 \\
\hline
\end{tabular}




\section{APPENDIX C - Additional Power Analyses}
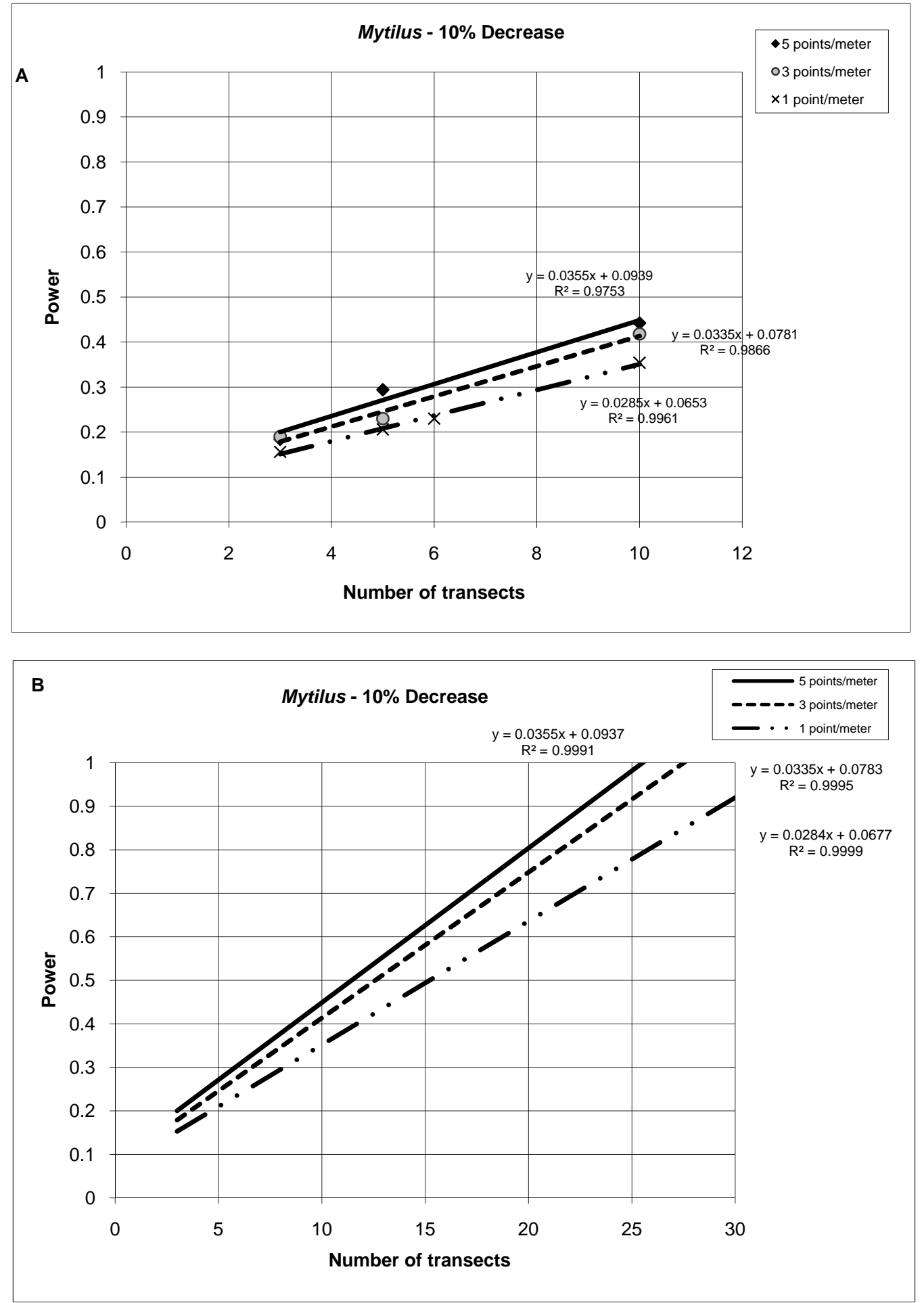

Figure $\mathrm{Cl}$ Change in the power to detect a $10 \%$ decrease in Mytilus as a result of varying the number of transects sampled per site with respect to 1,3 , and 5 points sampled per meter. Sampling was conducted in 1997 at 6 sites. $\alpha=0.05$. A. Points graphed using data. B. Linear trend of power based on data. 

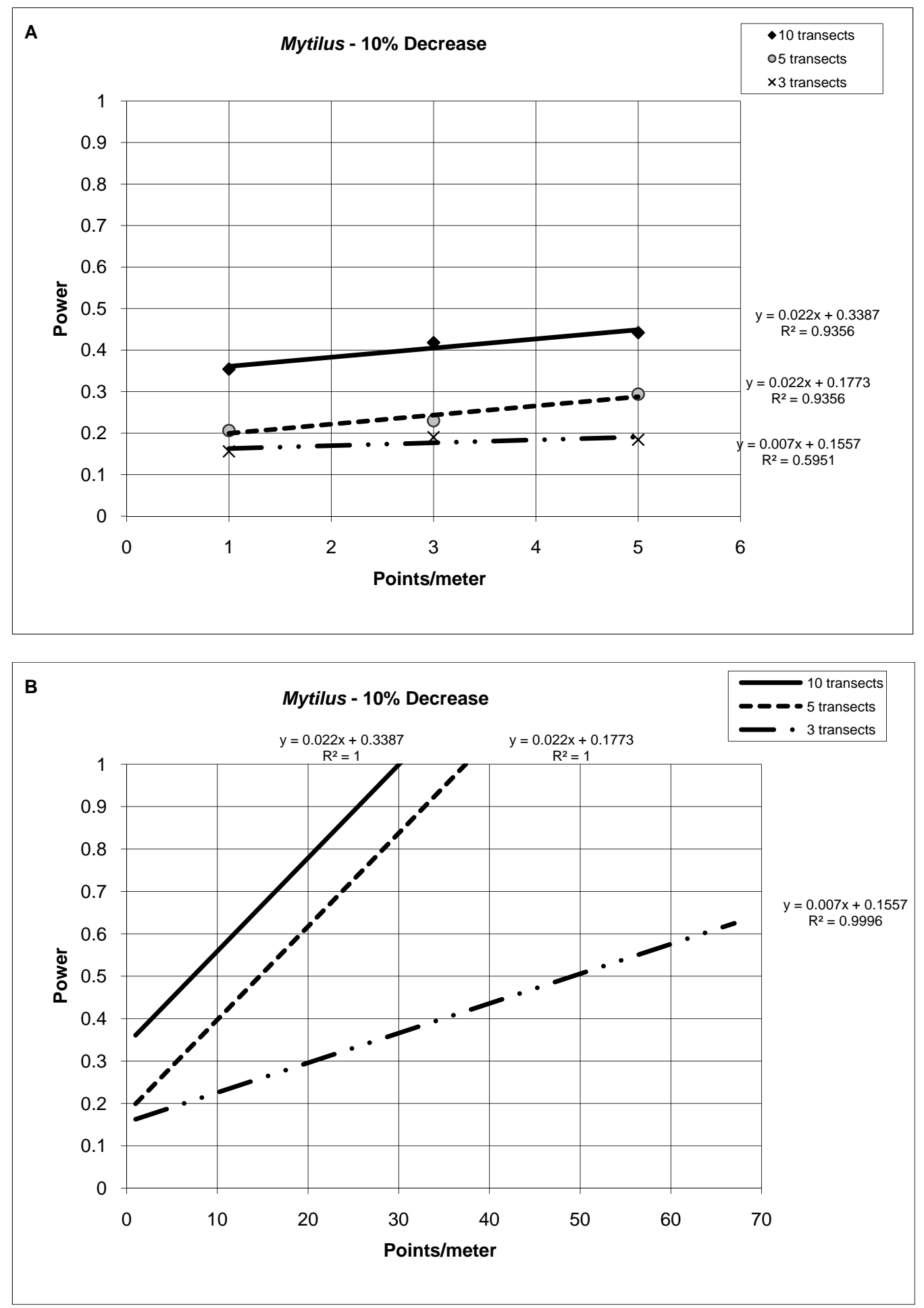

Figure C2. Change in the power to detect a $10 \%$ decrease in Mytilus as a result of varying the number of points sampled per meter with respect to 3, 5, and 10 transects per site. Sampling was conducted in 1997 at 6 sites. $\alpha=0.05$. A. Points graphed using data. B. Linear trend of power based on data. 

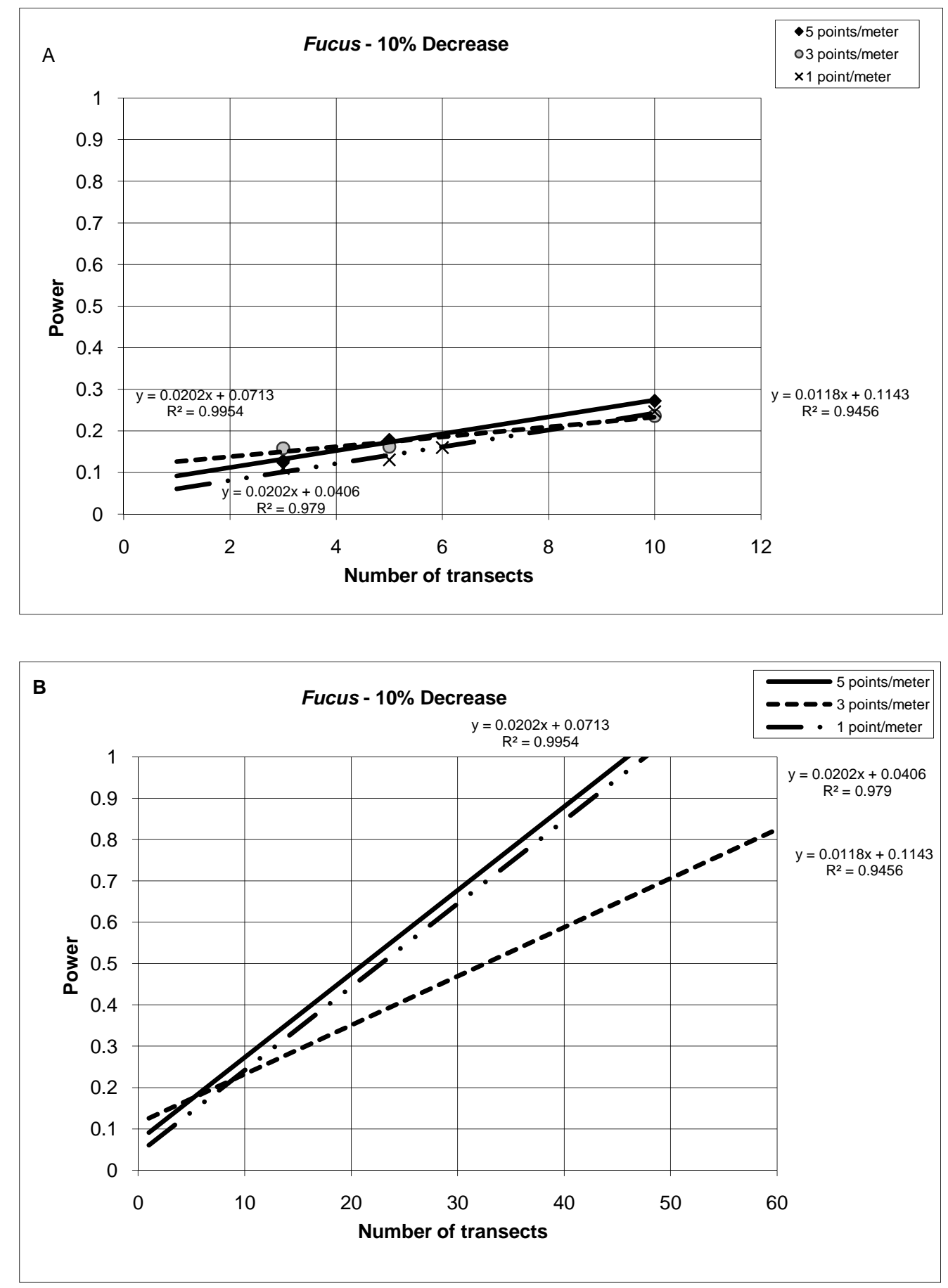

Figure C3. Change in the power to detect a 10\% decrease in Fucus as a result of varying the number of transects sampled per site with respect to 1,3 , and 5 points sampled per meter. Sampling was done in 1997 at 6 sites. $a=0.05$. A. Points graphed using data. B. Linear trend of power based on data. 

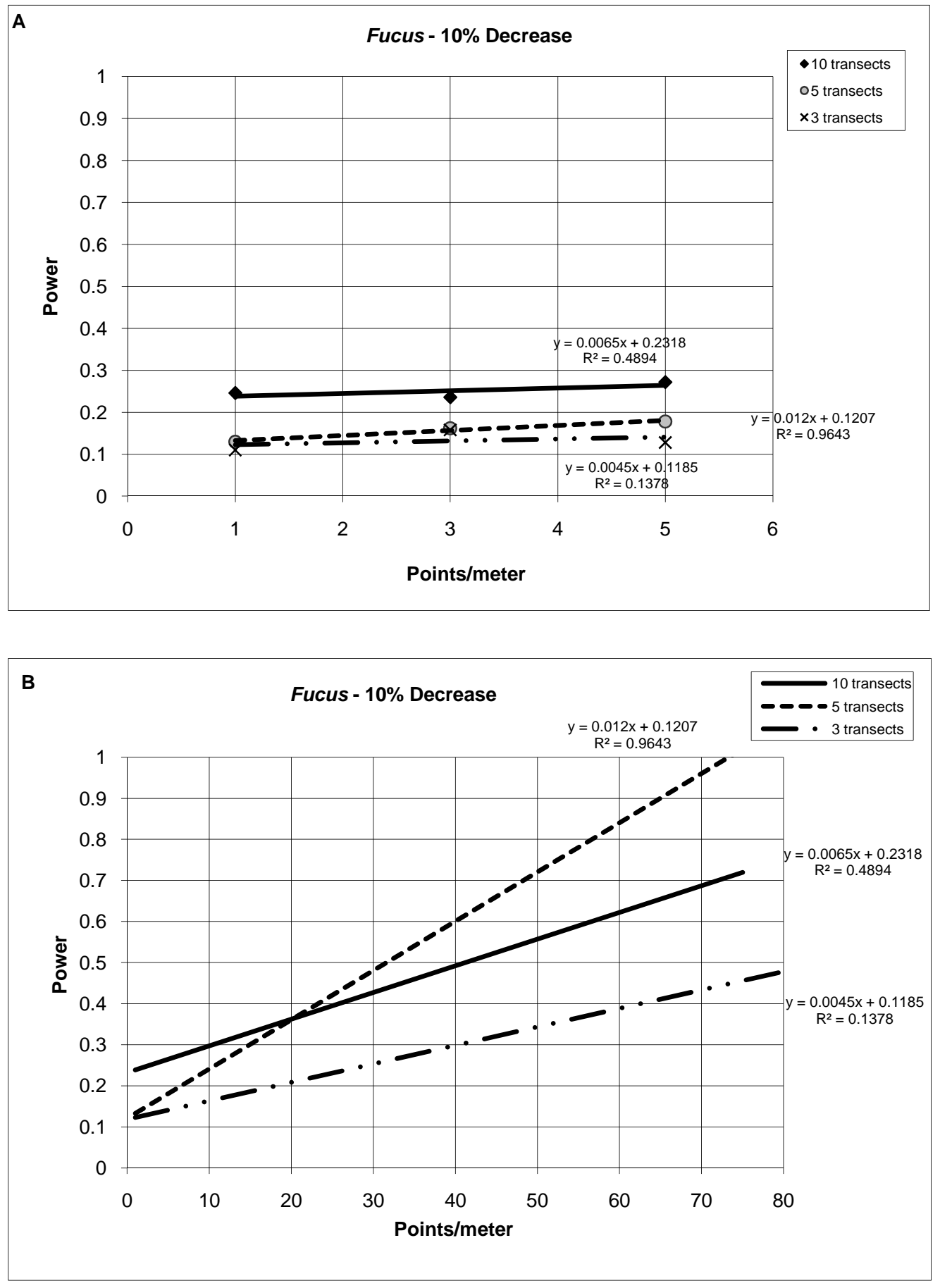

Figure C4. Change in the power to detect a 10\% decrease in Fucus as a result of varying the number of points sampled per meter with respect to 3, 5, and 10 transects per site. Sampling was done in 1997 at 6 sites. $a=0.05$. A. Points graphed using data. $B$. Linear trend of power based on data. 

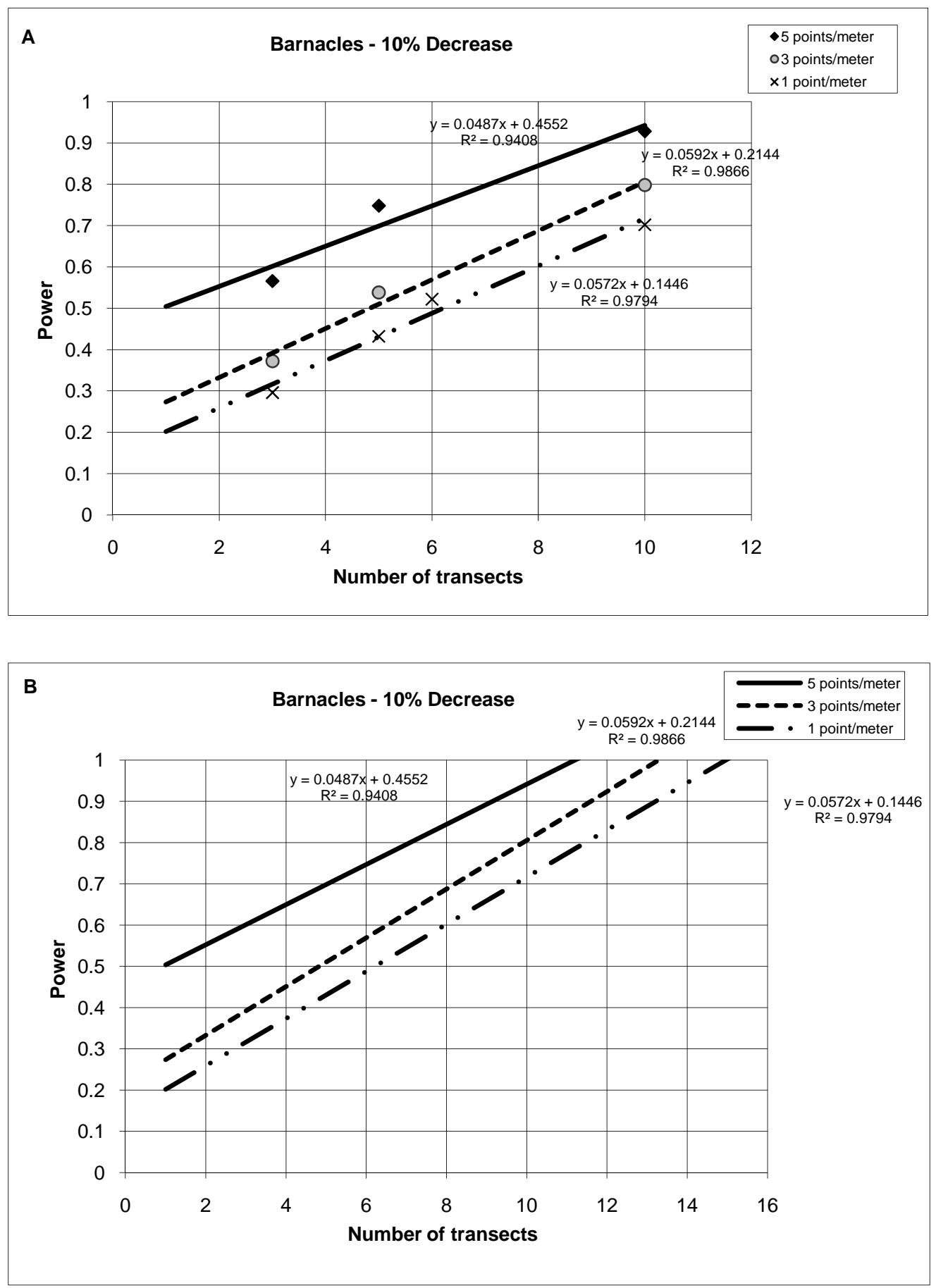

Figure $\mathbf{C 5}$. Change in the power to detect a $10 \%$ decrease in barnacles as a result of varying the number of transects sampled per site with respect to 1,3 , and 5 points sampled per meter. Sampling was done in 1997 at 6 sites. $a=0.05$. A. Points graphed using data. B. Linear trend of power based on data. 

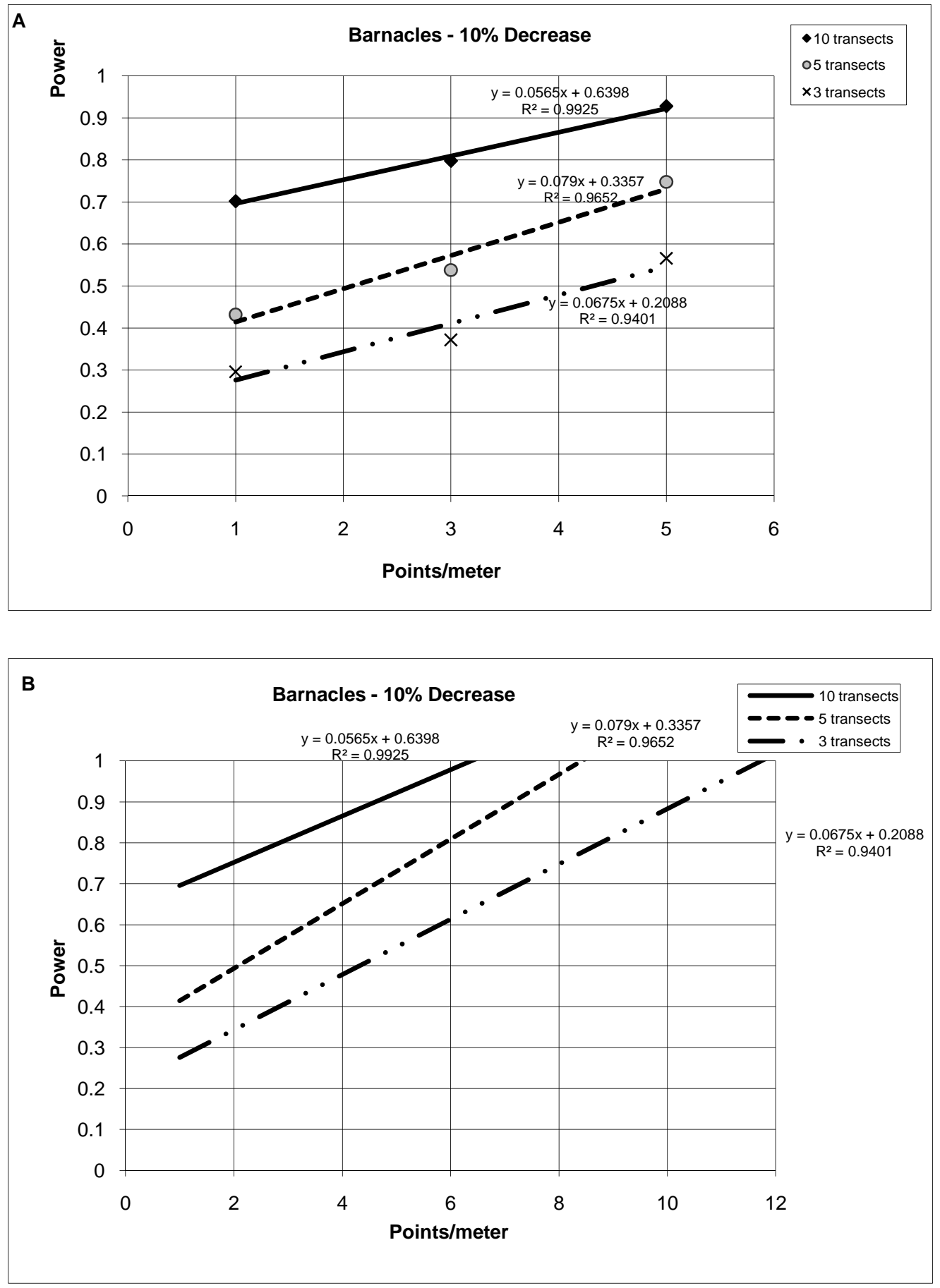

Figure C6. Change in the power to detect a $10 \%$ decrease in barnacles as a result of varying the number of points sampled per meter with respect to 3,5, and 10 transects per site. Sampling was done in 1997 at 6 sites. $a=0.05$. A. Points graphed using data. $B$. Linear trend of power based on data. 

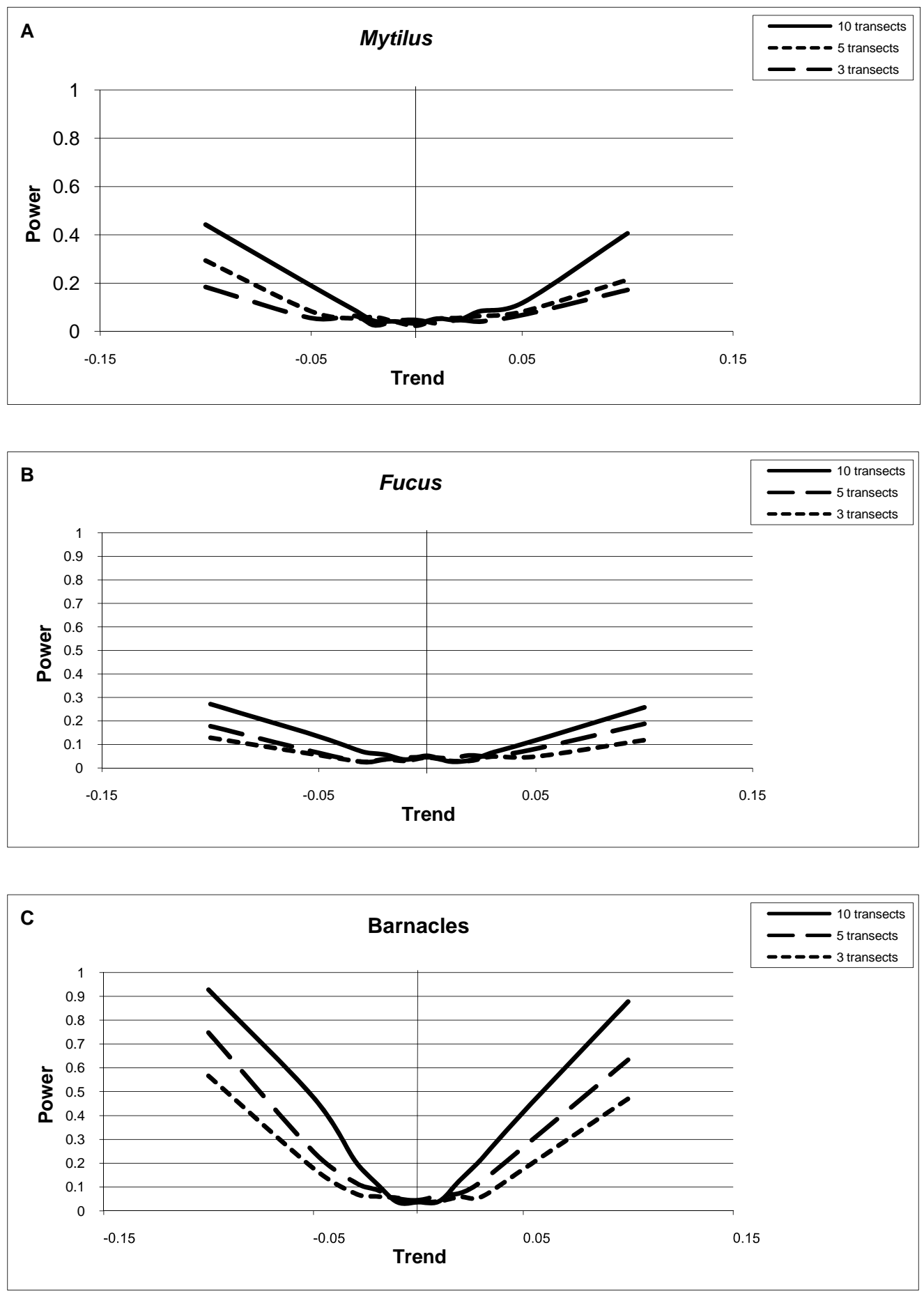

Figure C7. Power to detect trends of differing magnitudes at 3, 5, and 10 transects sampled per site. Data are based on the 1997 fine-grained sampling of 6 sites. Each transect is sampled at 5 points/meter. $a=$ 0.05. A. Mytilus; B. Fucus; C. Barnacles. 
Barnacles

\begin{tabular}{cr}
\hline Population Trend & \multicolumn{1}{c}{ Power } \\
\hline$-10 \%$ & 1 \\
$-5 \%$ & 0.82 \\
$-3 \%$ & 0.446 \\
$-2 \%$ & 0.242 \\
$-1 \%$ & 0.134 \\
$0 \%$ & 0.078 \\
$1 \%$ & 0.142 \\
$2 \%$ & 0.258 \\
$3 \%$ & 0.404 \\
$5 \%$ & 0.786 \\
$10 \%$ & 0.998
\end{tabular}

\section{Mytilus}

\begin{tabular}{cr}
\hline Population Trend & \multicolumn{1}{c}{ Power } \\
\hline$-10 \%$ & 0.996 \\
$-5 \%$ & 0.75 \\
$-3 \%$ & 0.382 \\
$-2 \%$ & 0.178 \\
$-1 \%$ & 0.106 \\
$0 \%$ & 0.1 \\
$1 \%$ & 0.114 \\
$2 \%$ & 0.222 \\
$3 \%$ & 0.366 \\
$5 \%$ & 0.694 \\
$10 \%$ & 0.996
\end{tabular}

\section{Fucus}

\begin{tabular}{cr}
\hline Population Trend & Power \\
\hline$-10 \%$ & 0.93 \\
$-5 \%$ & 0.398 \\
$-3 \%$ & 0.244 \\
$-2 \%$ & 0.142 \\
$-1 \%$ & 0.11 \\
$0 \%$ & 0.076 \\
$1 \%$ & 0.114 \\
$2 \%$ & 0.154 \\
$3 \%$ & 0.228 \\
$5 \%$ & 0.426 \\
$10 \%$ & 0.868
\end{tabular}
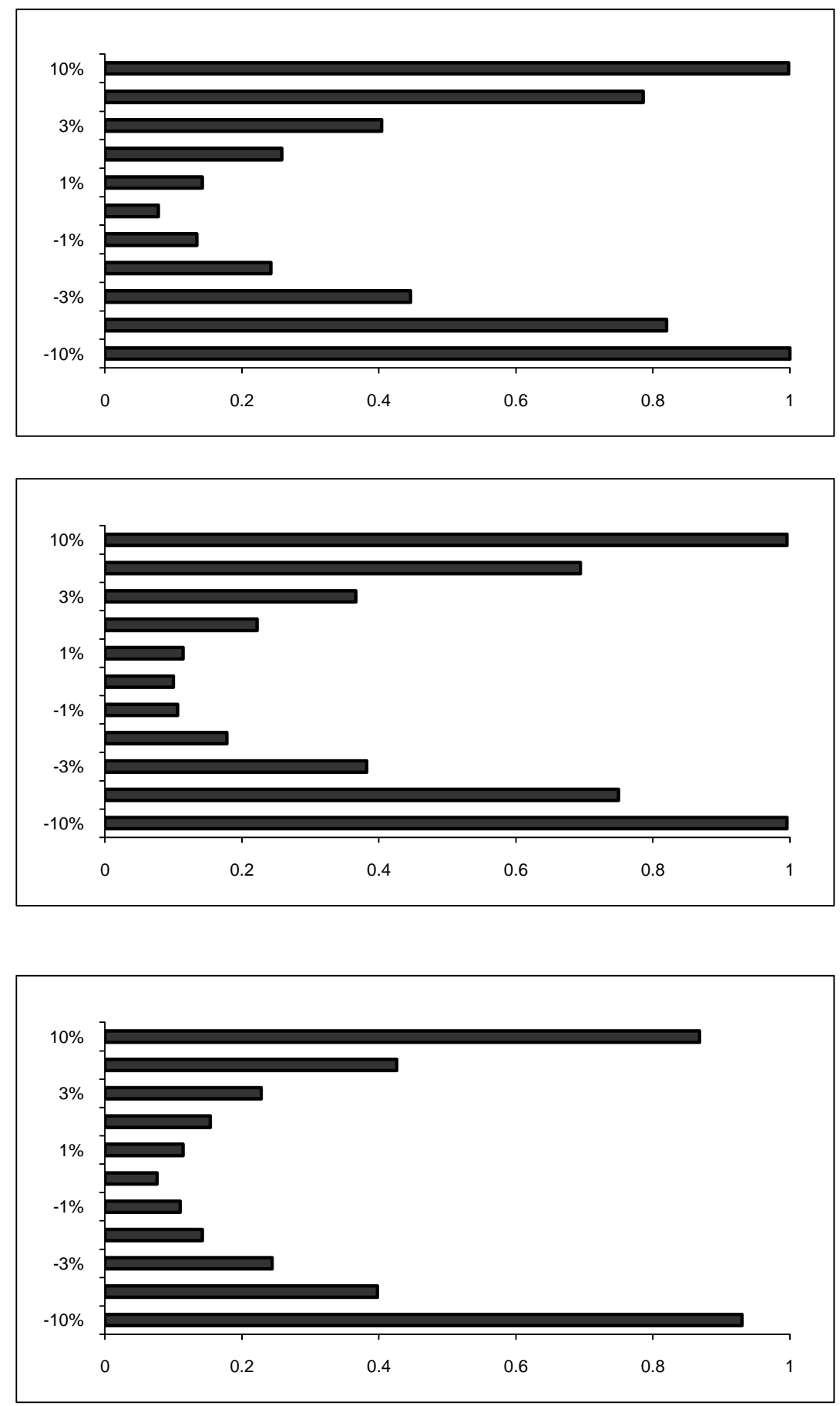

Figure C8. Power to detect population trends based on 3 years of data (1998-2001) in A. barnacles; B. Mytilus, and C. Fucus. Sampling was done on the level of 25 sites, 6 transects, 1 point/meter. $a=0.10$. 


\section{Barnacles}

\begin{tabular}{cr}
\hline Population Trend & Power \\
\hline$-10 \%$ & 0.998 \\
$-5 \%$ & 0.796 \\
$-3 \%$ & 0.45 \\
$-2 \%$ & 0.278 \\
$-1 \%$ & 0.176 \\
$0 \%$ & 0.1 \\
$1 \%$ & 0.144 \\
$2 \%$ & 0.276 \\
$3 \%$ & 0.424 \\
$5 \%$ & 0.798 \\
$10 \%$ & 0.994
\end{tabular}

\section{Mytilus}

\begin{tabular}{cr}
\hline Population Trend & \multicolumn{1}{c}{ Power } \\
\hline$-10 \%$ & 1 \\
$-5 \%$ & 0.808 \\
$-3 \%$ & 0.468 \\
$-2 \%$ & 0.258 \\
$-1 \%$ & 0.154 \\
$0 \%$ & 0.124 \\
$1 \%$ & 0.144 \\
$2 \%$ & 0.228 \\
$3 \%$ & 0.416 \\
$5 \%$ & 0.75 \\
$10 \%$ & 0.996
\end{tabular}

\section{Fucus}

\begin{tabular}{cr}
\hline Population Trend & Power \\
\hline$-10 \%$ & 0.95 \\
$-5 \%$ & 0.46 \\
$-3 \%$ & 0.266 \\
$-2 \%$ & 0.212 \\
$-1 \%$ & 0.12 \\
$0 \%$ & 0.098 \\
$1 \%$ & 0.114 \\
$2 \%$ & 0.182 \\
$3 \%$ & 0.234 \\
$5 \%$ & 0.492 \\
$10 \%$ & 0.88
\end{tabular}
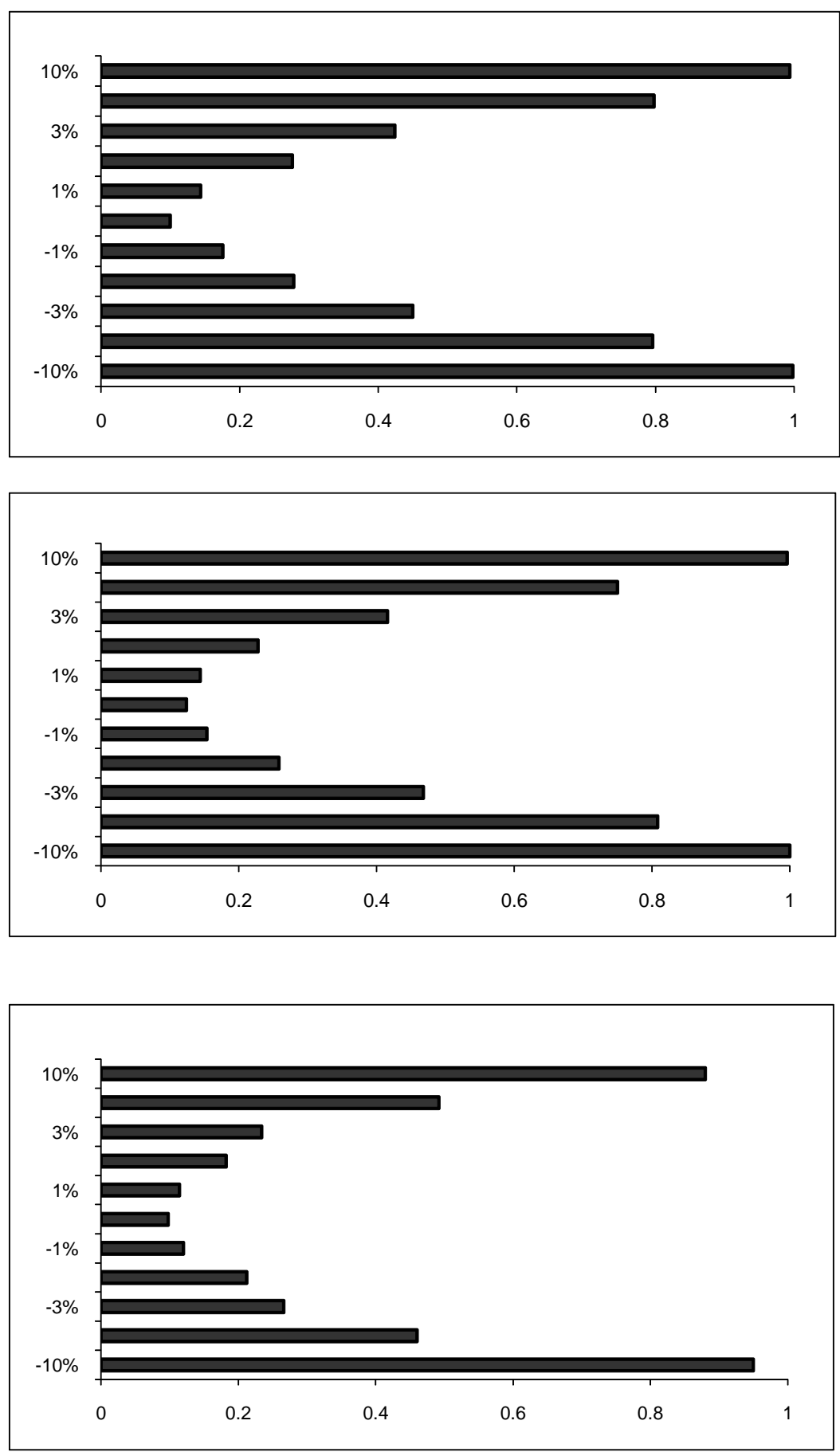

Figure C9. Power to detect population trends based on 3 years of data (1998-2001) in A. barnacles; B. Mytilus; and C. Fucus. Sampling was done on the level of 25 sites, 6 transects, 2 points/meter. $\alpha=0.10$. 
Barnacles

\begin{tabular}{cr}
\hline Population Trend & \multicolumn{1}{c}{ Power } \\
\hline$-10 \%$ & 1 \\
$-5 \%$ & 0.84 \\
$-3 \%$ & 0.452 \\
$-2 \%$ & 0.294 \\
$-1 \%$ & 0.132 \\
$-0 \%$ & 0.122 \\
$1 \%$ & 0.15 \\
$2 \%$ & 0.28 \\
$3 \%$ & 0.45 \\
$5 \%$ & 0.8 \\
$10 \%$ & 1
\end{tabular}

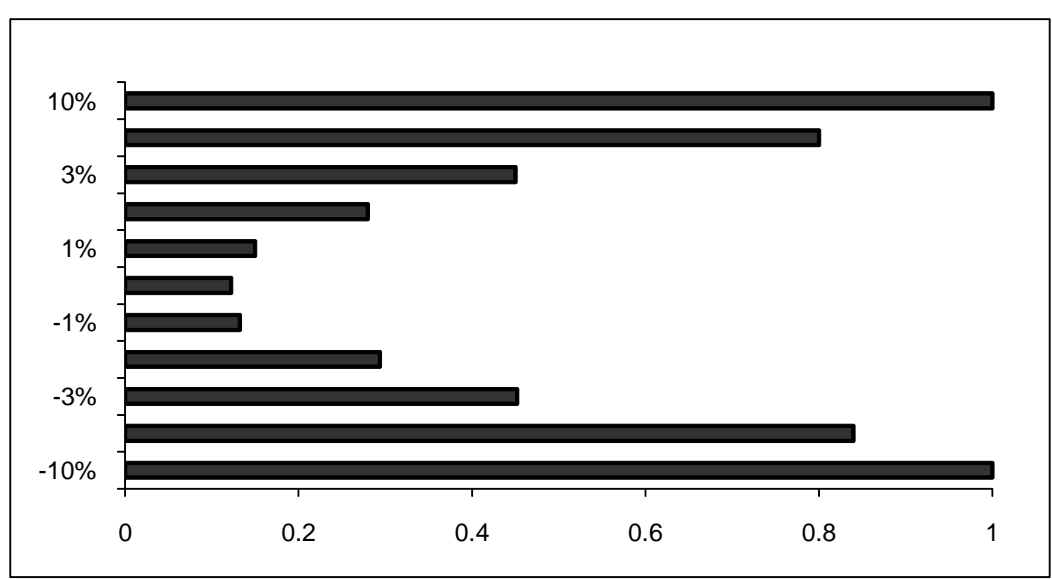

\section{Mytilus}

\begin{tabular}{cr}
\hline Population Trend & \multicolumn{1}{c}{ Power } \\
\hline$-10 \%$ & 0.998 \\
$-5 \%$ & 0.888 \\
$-3 \%$ & 0.526 \\
$-2 \%$ & 0.29 \\
$-1 \%$ & 0.154 \\
$-0 \%$ & 0.094 \\
$1 \%$ & 0.162 \\
$2 \%$ & 0.286 \\
$3 \%$ & 0.474 \\
$5 \%$ & 0.83 \\
$10 \%$ & 1
\end{tabular}

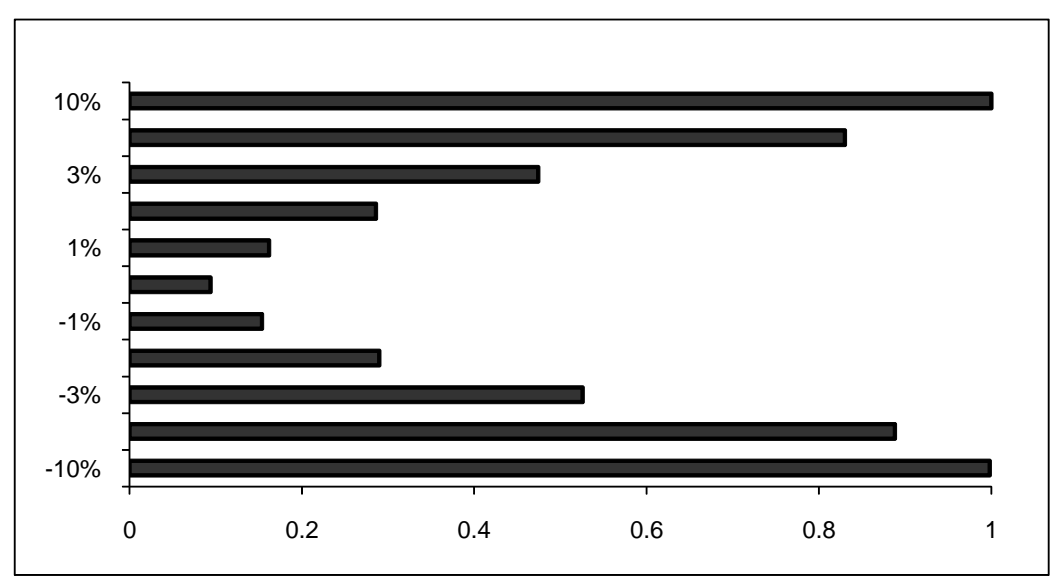

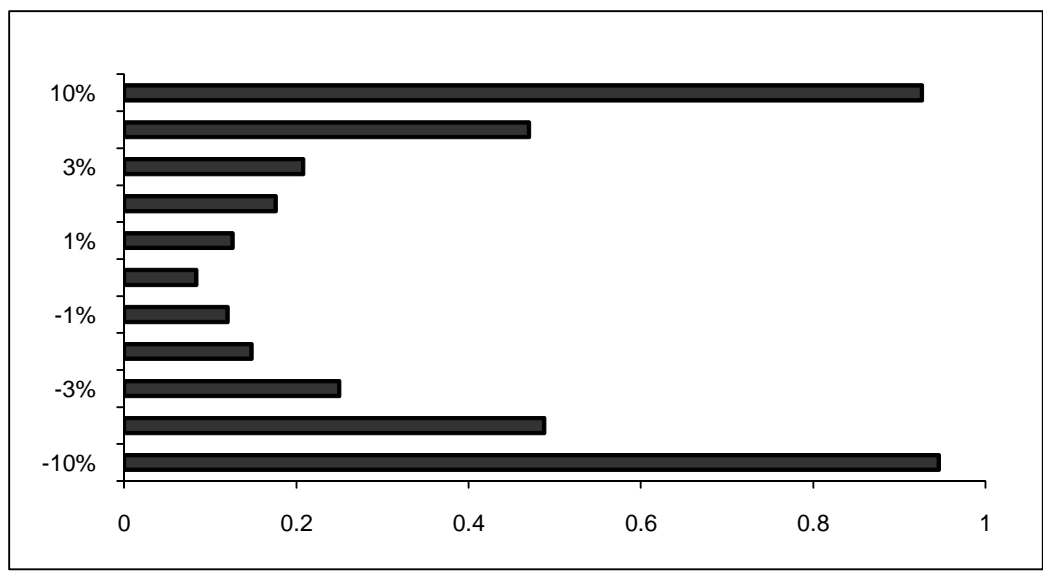

Fucus

\begin{tabular}{cr}
\hline Population Trend & Power \\
\hline$-10 \%$ & 0.946 \\
$-5 \%$ & 0.488 \\
$-3 \%$ & 0.25 \\
$-2 \%$ & 0.148 \\
$-1 \%$ & 0.12 \\
$-0 \%$ & 0.084 \\
$1 \%$ & 0.126 \\
$2 \%$ & 0.176 \\
$3 \%$ & 0.208 \\
$5 \%$ & 0.47 \\
$10 \%$ & 0.926
\end{tabular}


Barnacles

\begin{tabular}{cr}
\hline Population Trend & \multicolumn{1}{c}{ Power } \\
\hline$-10 \%$ & 1 \\
$-5 \%$ & 0.828 \\
$-3 \%$ & 0.498 \\
$-2 \%$ & 0.288 \\
$-1 \%$ & 0.154 \\
$-0 \%$ & 0.136 \\
$1 \%$ & 0.152 \\
$2 \%$ & 0.238 \\
$3 \%$ & 0.414 \\
$5 \%$ & 0.816 \\
$10 \%$ & 0.998
\end{tabular}

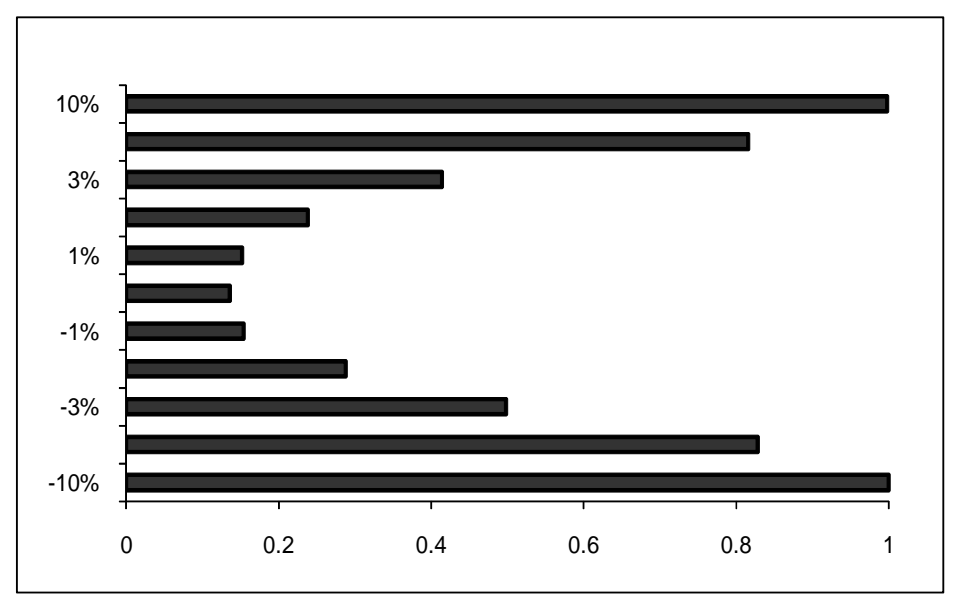

Mytilus

\begin{tabular}{lr}
\hline Population Trend & \multicolumn{1}{r}{ Power } \\
\hline$-10 \%$ & 1 \\
$-5 \%$ & 0.894 \\
$-3 \%$ & 0.532 \\
$-2 \%$ & 0.304 \\
$-1 \%$ & 0.152 \\
$-0 \%$ & 0.094 \\
$1 \%$ & 0.188 \\
$2 \%$ & 0.302 \\
$3 \%$ & 0.506 \\
$5 \%$ & 0.894 \\
$10 \%$ & 1
\end{tabular}

\section{Fucus}

\begin{tabular}{cr}
\hline Population Trend & Power \\
\hline$-10 \%$ & 0.948 \\
$-5 \%$ & 0.47 \\
$-3 \%$ & 0.246 \\
$-2 \%$ & 0.184 \\
$-1 \%$ & 0.1 \\
$-0 \%$ & 0.082 \\
$1 \%$ & 0.136 \\
$2 \%$ & 0.17 \\
$3 \%$ & 0.292 \\
$5 \%$ & 0.48 \\
$10 \%$ & 0.898
\end{tabular}
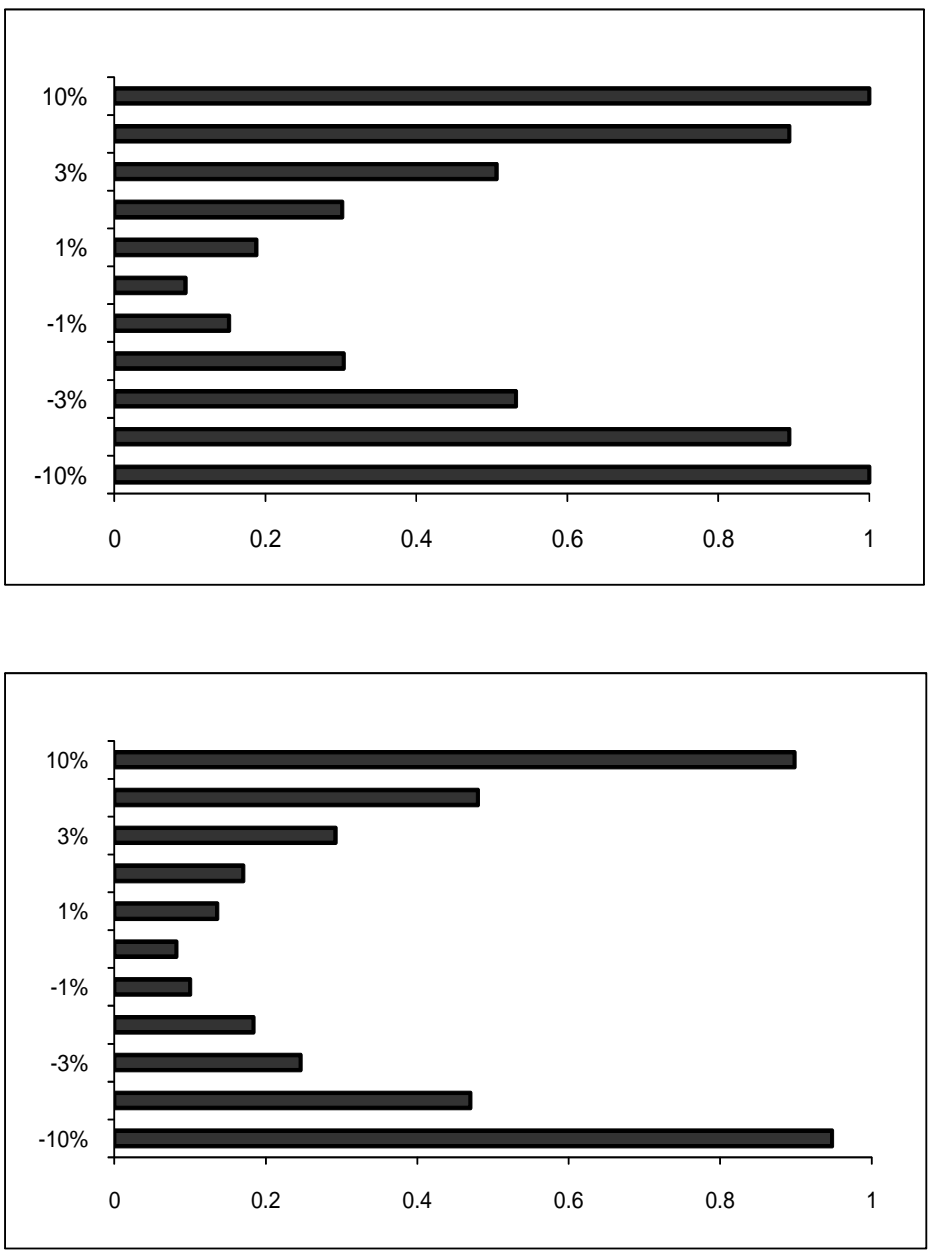

Figure C11 Power to detect population trends based on 3 years of data (1998-2001) in A. barnacles; $B$. Mytilus, and C. Fucus. Sampling was done on the level of 25 sites, 6 transects, 4 points/meter. $a=0.10$. 
Barnacles

\begin{tabular}{cr}
\hline Population Trend & \multicolumn{1}{c}{ Power } \\
\hline$-10 \%$ & 1 \\
$-5 \%$ & 0.868 \\
$-3 \%$ & 0.492 \\
$-2 \%$ & 0.29 \\
$-1 \%$ & 0.15 \\
$-0 \%$ & 0.116 \\
$1 \%$ & 0.14 \\
$2 \%$ & 0.256 \\
$3 \%$ & 0.466 \\
$5 \%$ & 0.808 \\
$10 \%$ & 1
\end{tabular}

Mytilus

\begin{tabular}{lr}
\hline Population Trend & \multicolumn{1}{c}{ Power } \\
\hline$-10 \%$ & 1 \\
$-5 \%$ & 0.914 \\
$-3 \%$ & 0.564 \\
$-2 \%$ & 0.306 \\
$-1 \%$ & 0.166 \\
$-0 \%$ & 0.088 \\
$1 \%$ & 0.134 \\
$2 \%$ & 0.316 \\
$3 \%$ & 0.528 \\
$5 \%$ & 0.834 \\
$10 \%$ & 1
\end{tabular}

\section{Fucus}

\begin{tabular}{cr}
\multicolumn{2}{l}{ Fucus } \\
\hline Population Trend & Power \\
\hline$-10 \%$ & 0.946 \\
$-5 \%$ & 0.508 \\
$-3 \%$ & 0.274 \\
$-2 \%$ & 0.142 \\
$-1 \%$ & 0.14 \\
$-0 \%$ & 0.084 \\
$1 \%$ & 0.102 \\
$2 \%$ & 0.138 \\
$3 \%$ & 0.268 \\
$5 \%$ & 0.474 \\
$10 \%$ & 0.922
\end{tabular}
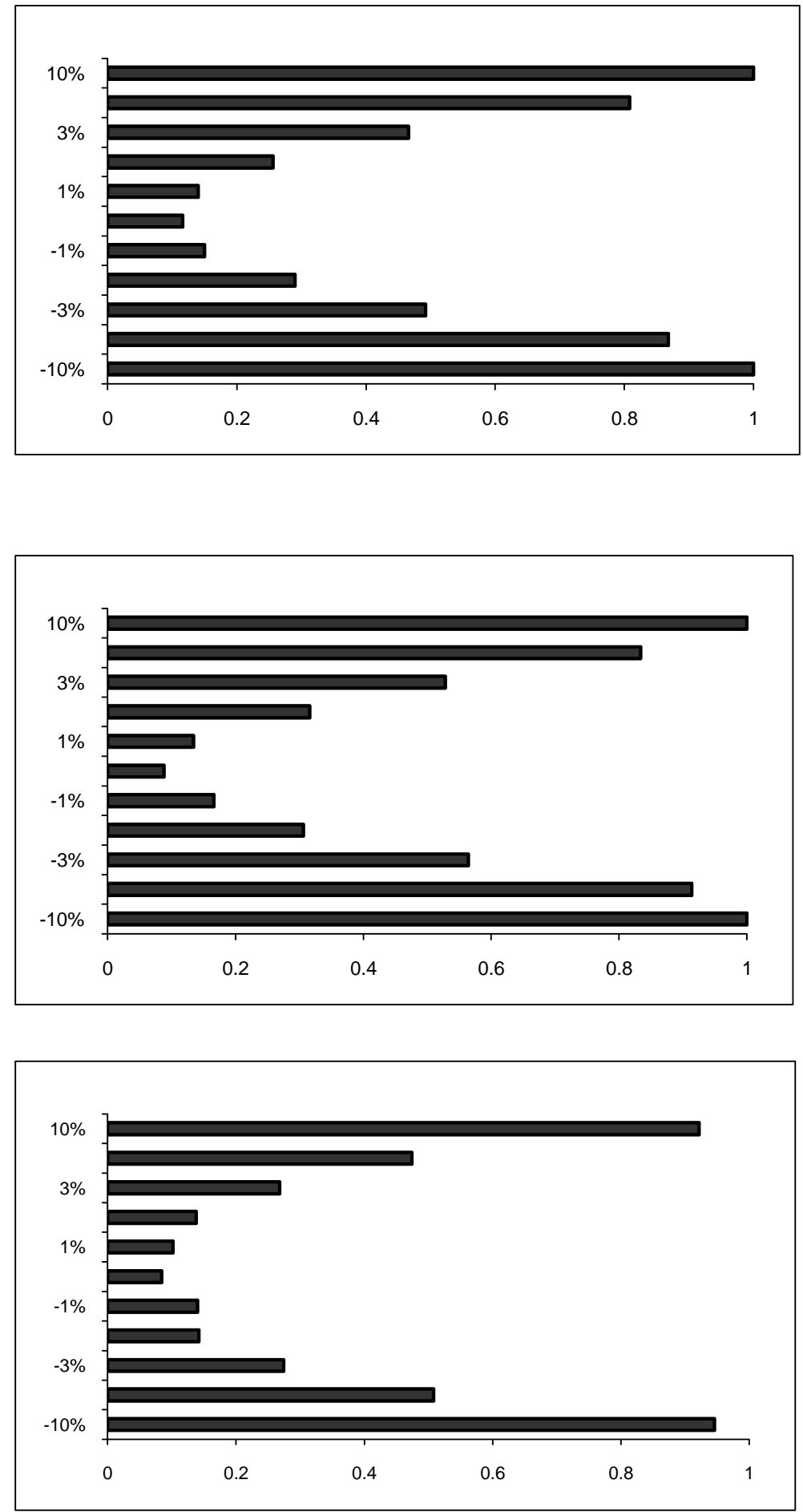

Figure C12. Power to detect population trends based on 3 years of data (1998-2001) in A. barnacles; B. Mytilus; and C. Fucus. Sampling was done on the level of 25 sites, 6 transects, 5 points/meter. $\alpha=0.10$. 

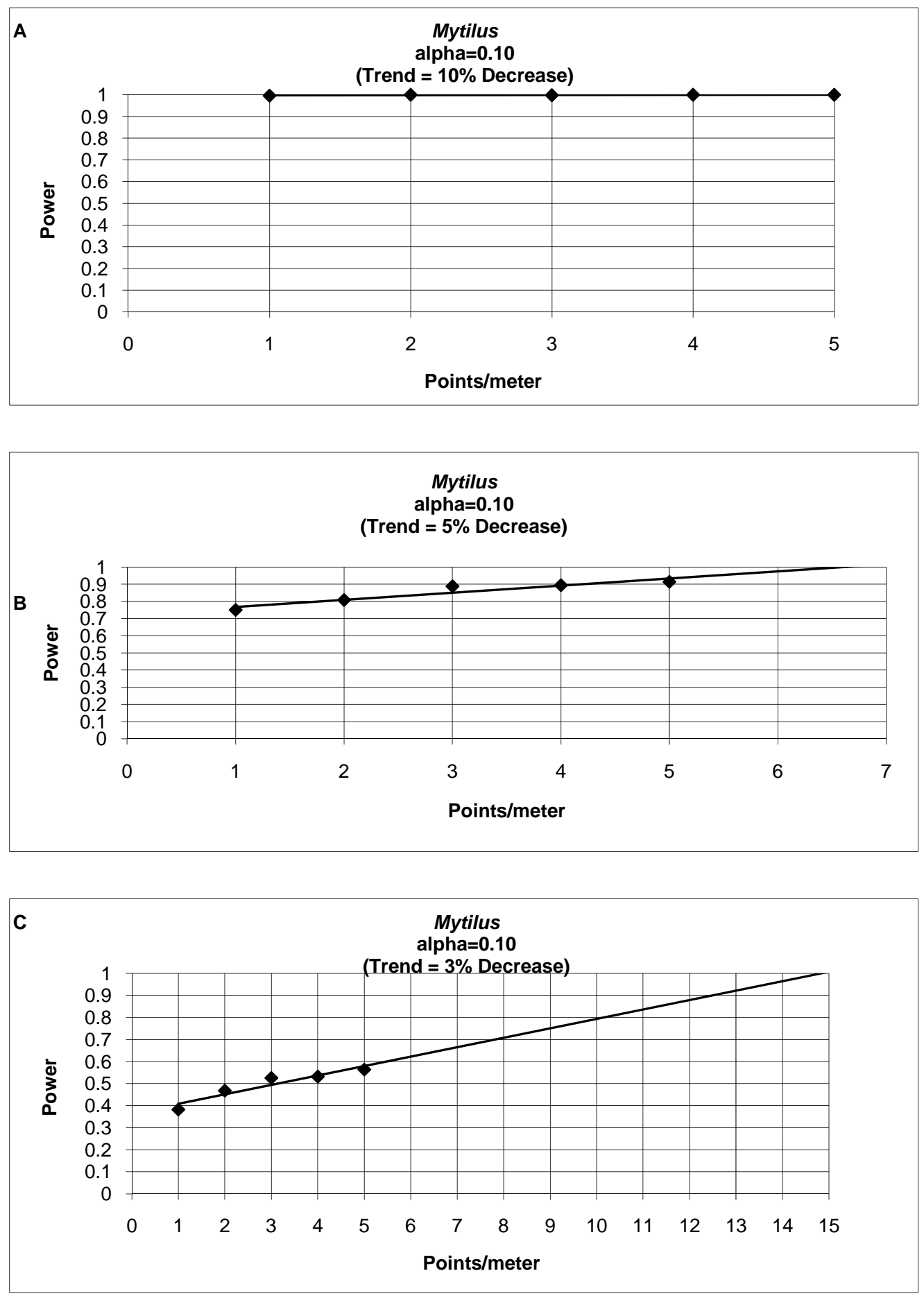

Figure C13. Power to detect a decline in Mytilus populations as a function of the number of points sampled per meter of each transect. Data are based on 3 years of sampling (1998-2001) at 25 sites, 6 transects at each site. $\alpha=0.10$. A. $10 \%$ decrease; B. $5 \%$ decrease; C. $3 \%$ decrease. Points on graphs indicate data; lines are linear projections of the trends suggested by these data. 

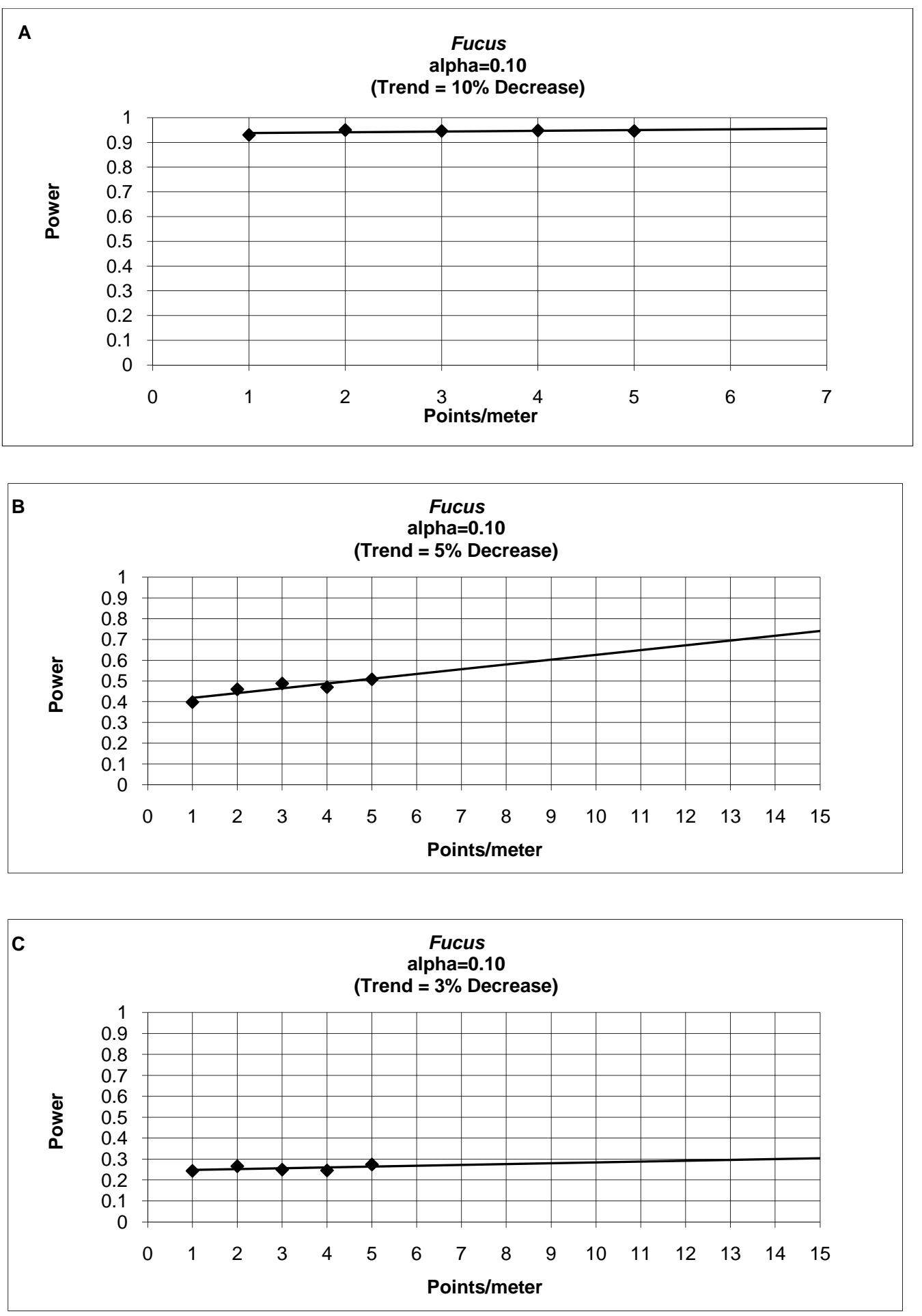

Figure C14. Power to detect a decline in Fucus populations as a function of the number of points sampled per meter of each transect. Data are based on 3 years of sampling (1998-2001) at 25 sites, 6 transects at each site. $a=0.10$. A. $10 \%$ decrease; B. $5 \%$ decrease; C. $3 \%$ decrease. Points on graphs indicate data; lines are linear projections of the trends suggested by these data. 

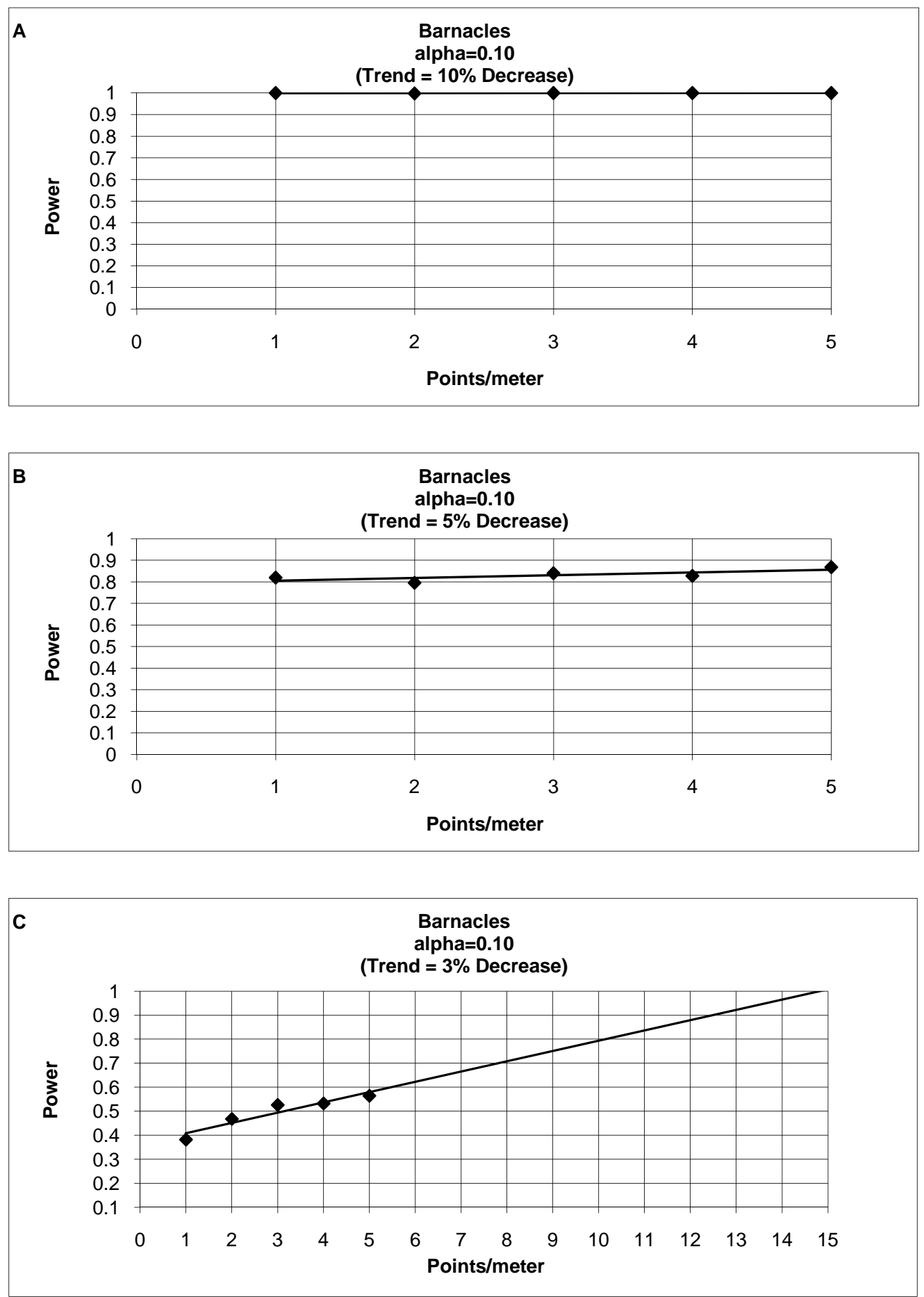

Figure C15. Power to detect a decline in barnacle populations as a function of the number of points sampled per meter of each transect. Data are based on 3 years of sampling (1998-2001) at 25 sites, 6 transects at each site. $\alpha=0.10$. A. $10 \%$ decrease; B. $5 \%$ decrease; C. 3\% decrease. Points on graphs indicate data; lines are linear projections of the trends suggested by these data. 

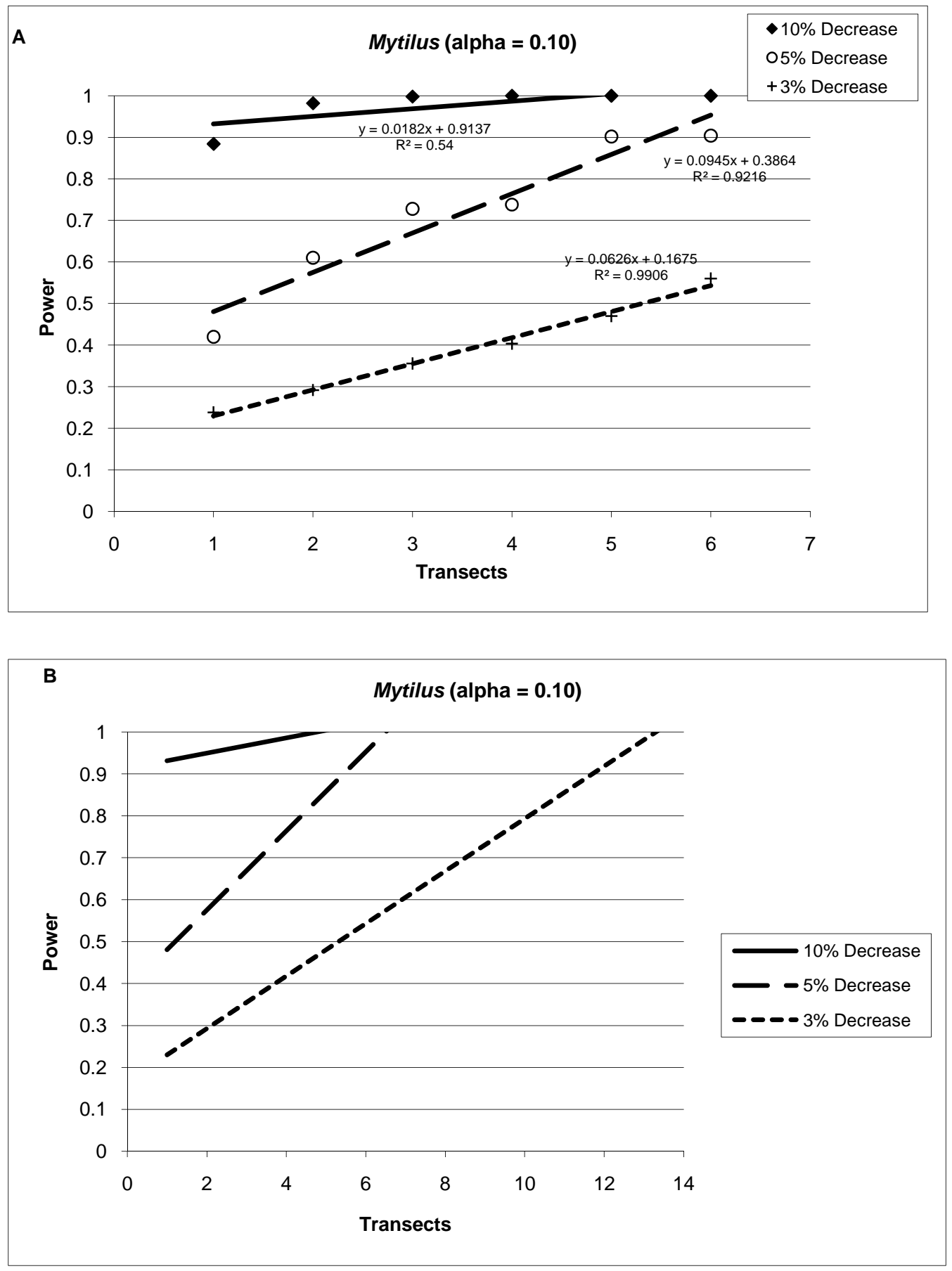

Figure C16. Power to detect declines in Mytilus populations (10\%, $5 \%$, and $3 \%)$ as a function of the number of transects sampled at each site. Transects were sampled at a frequency of 1 point/meter. Data are based on the analysis of 25 sites sampled 4 times (1997-2001). $\alpha=0.10$. A. Points graphed using data. B. Linear trend of power based on data. 
Barnacles

\begin{tabular}{cc}
\hline Population Trend & Power \\
\hline$-10 \%$ & 1 \\
$-5 \%$ & 0.9 \\
$-3 \%$ & 0.6 \\
$-2 \%$ & 0.416 \\
$-1 \%$ & 0.274 \\
$-0 \%$ & 0.214 \\
$1 \%$ & 0.238 \\
$2 \%$ & 0.444 \\
$3 \%$ & 0.584 \\
$5 \%$ & 0.884 \\
$10 \%$ & 1
\end{tabular}

\section{Mytilus}

\begin{tabular}{cc}
\hline Population Trend & Power \\
\hline$-10 \%$ & 1 \\
$-5 \%$ & 0.838 \\
$-3 \%$ & 0.524 \\
$-2 \%$ & 0.334 \\
$-1 \%$ & 0.248 \\
$-0 \%$ & 0.194 \\
$1 \%$ & 0.24 \\
$2 \%$ & 0.368 \\
$3 \%$ & 0.496 \\
$5 \%$ & 0.816 \\
$10 \%$ & 0.998
\end{tabular}

\section{Fucus}

\begin{tabular}{cc}
\hline Population Trend & Power \\
\hline$-10 \%$ & 0.982 \\
$-5 \%$ & 0.632 \\
$-3 \%$ & 0.382 \\
$-2 \%$ & 0.264 \\
$-1 \%$ & 0.206 \\
$-0 \%$ & 0.21 \\
$1 \%$ & 0.244 \\
$2 \%$ & 0.26 \\
$3 \%$ & 0.34 \\
$5 \%$ & 0.548 \\
$10 \%$ & 0.928
\end{tabular}
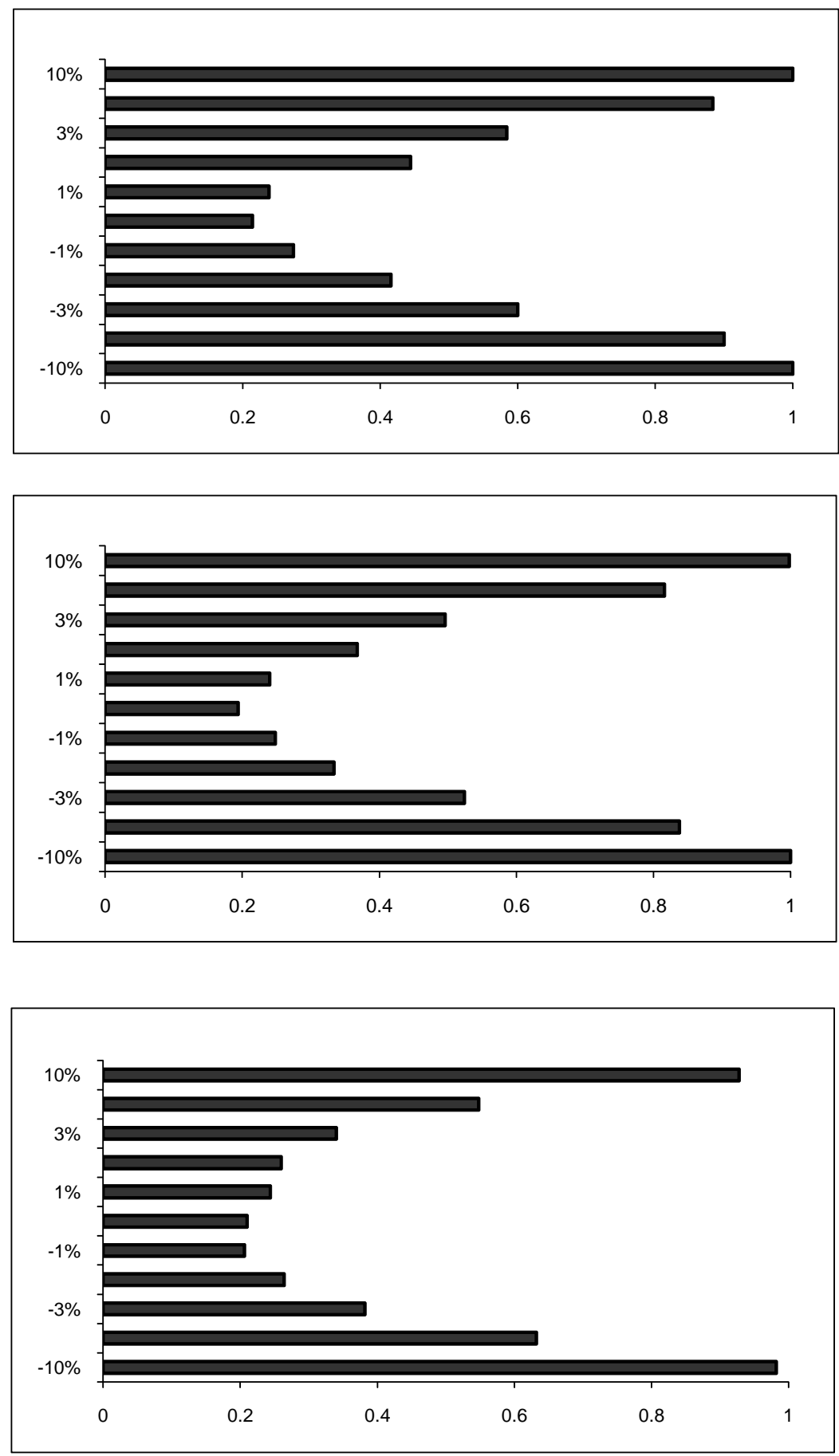

Figure C17. Power Power to detect population trends based on 3 years of data (1998-2001) in A. barnacles; B. Mytilus; and C. Fucus. Sampling was done on the level of 25 sites, 6 transects, 1 point/meter. $a=0.20$. 
Barnacles

\begin{tabular}{cc}
\hline Population Trend & Power \\
\hline$-10 \%$ & 1 \\
$-5 \%$ & 0.906 \\
$-3 \%$ & 0.604 \\
$-2 \%$ & 0.376 \\
$-1 \%$ & 0.262 \\
$0 \%$ & 0.178 \\
$1 \%$ & 0.254 \\
$2 \%$ & 0.408 \\
$3 \%$ & 0.574 \\
$5 \%$ & 0.864 \\
$10 \%$ & 1
\end{tabular}

\section{Mytilus}

\begin{tabular}{cc}
\hline Population Trend & Power \\
\hline$-10 \%$ & 1 \\
$-5 \%$ & 0.904 \\
$-3 \%$ & 0.578 \\
$-2 \%$ & 0.376 \\
$-1 \%$ & 0.272 \\
$0 \%$ & 0.214 \\
$1 \%$ & 0.254 \\
$2 \%$ & 0.358 \\
$3 \%$ & 0.552 \\
$5 \%$ & 0.876 \\
$10 \%$ & 1
\end{tabular}

\section{Fucus}

\begin{tabular}{cc}
\hline Population Trend & Power \\
\hline$-10 \%$ & 0.976 \\
$-5 \%$ & 0.688 \\
$-3 \%$ & 0.384 \\
$-2 \%$ & 0.294 \\
$-1 \%$ & 0.2 \\
$0 \%$ & 0.22 \\
$1 \%$ & 0.208 \\
$2 \%$ & 0.304 \\
$3 \%$ & 0.386 \\
$5 \%$ & 0.572 \\
$10 \%$ & 0.958
\end{tabular}
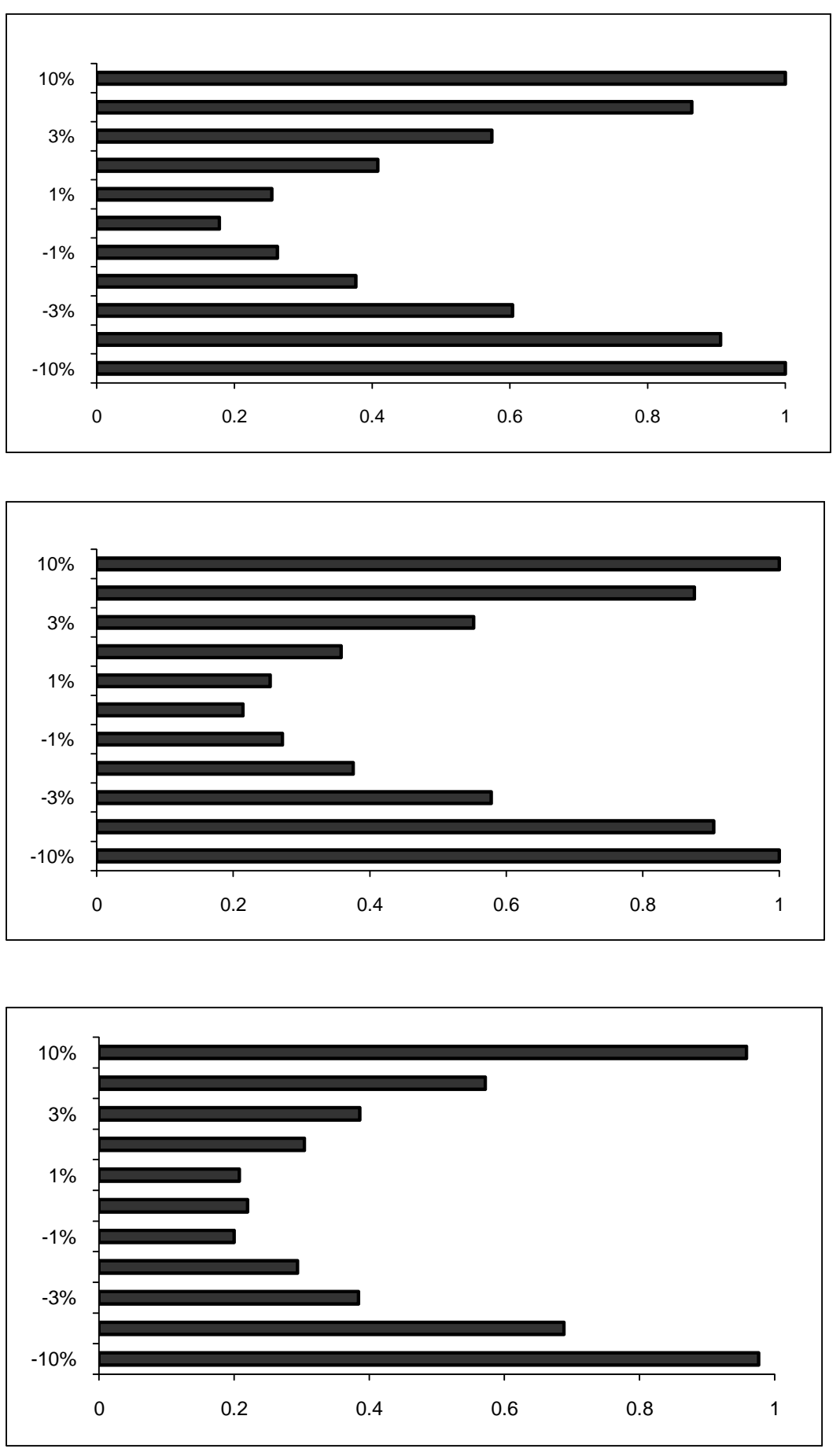

Figure C18. Power to detect population trends based on 3 years of data (1998-1999) in A. barnacles; B. Mytilus, and C. Fucus. Sampling was done on the level of 25 sites, 6 transects, 2 points/meter. $a=0.20$. 
Barnacles

\begin{tabular}{cc}
\hline Population Trend & Power \\
\hline$-10 \%$ & 1 \\
$-5 \%$ & 0.928 \\
$-3 \%$ & 0.606 \\
$-2 \%$ & 0.422 \\
$-1 \%$ & 0.248 \\
$-0 \%$ & 0.174 \\
$1 \%$ & 0.25 \\
$2 \%$ & 0.426 \\
$3 \%$ & 0.598 \\
$5 \%$ & 0.92 \\
$10 \%$ & 0.998
\end{tabular}

\section{Mytilus}

\begin{tabular}{cc}
\hline Population Trend & Power \\
\hline$-10 \%$ & 1 \\
$-5 \%$ & 0.926 \\
$-3 \%$ & 0.65 \\
$-2 \%$ & 0.45 \\
$-1 \%$ & 0.3 \\
$-0 \%$ & 0.194 \\
$1 \%$ & 0.268 \\
$2 \%$ & 0.504 \\
$3 \%$ & 0.62 \\
$5 \%$ & 0.926 \\
$10 \%$ & 1
\end{tabular}

\section{Fucus}

\begin{tabular}{cc}
\hline Population Trend & Power \\
\hline$-10 \%$ & 0.98 \\
$-5 \%$ & 0.67 \\
$-3 \%$ & 0.382 \\
$-2 \%$ & 0.304 \\
$-1 \%$ & 0.23 \\
$-0 \%$ & 0.216 \\
$1 \%$ & 0.218 \\
$2 \%$ & 0.292 \\
$3 \%$ & 0.39 \\
$5 \%$ & 0.576 \\
$10 \%$ & 0.98
\end{tabular}
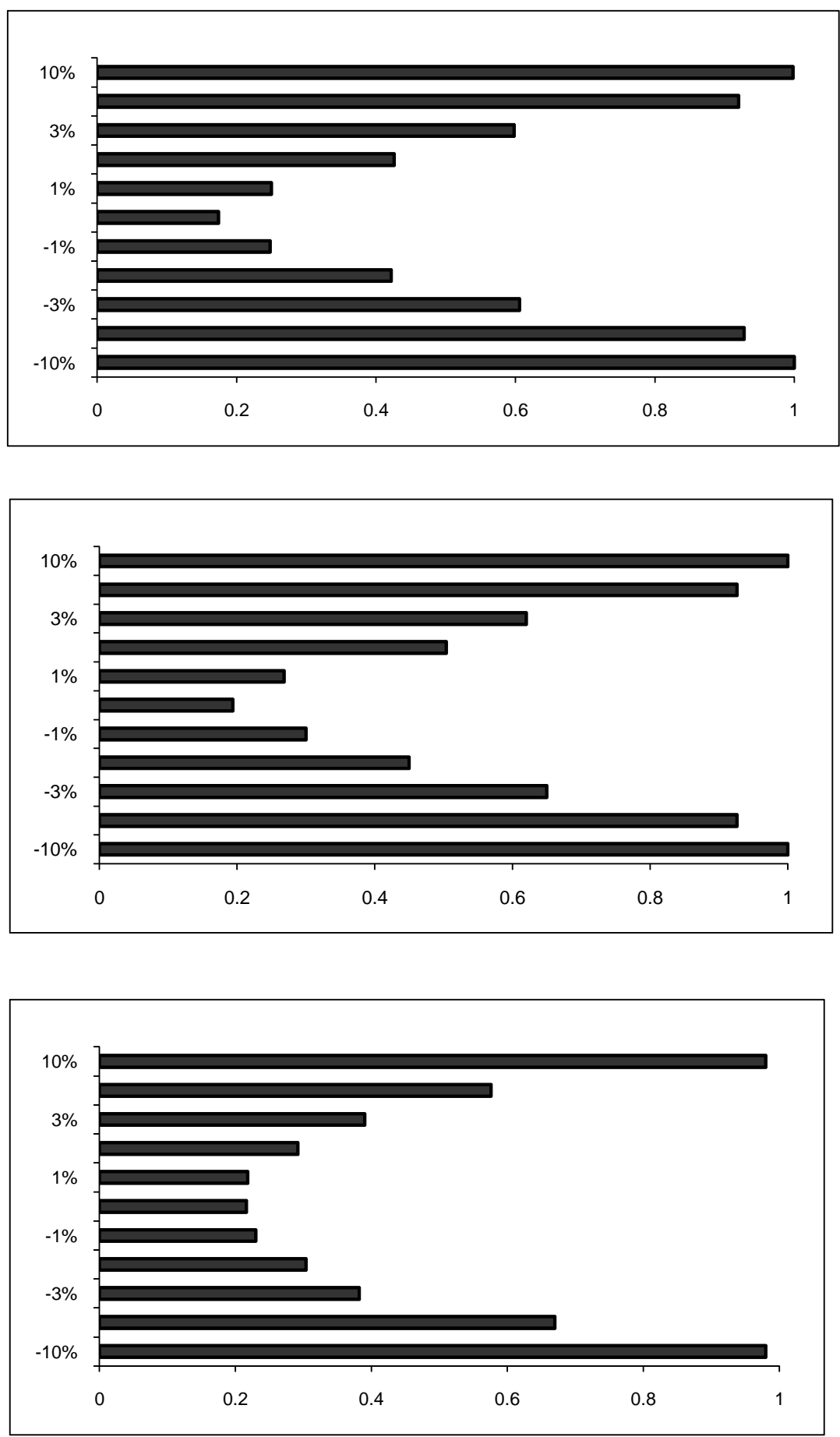

Figure C19. Power to detect population trends based on 3 years of data (1998-2001) in A. barnacles; B. Mytilus; and C. Fucus. Sampling was done on the level of 25 sites, 6 transects, 3 points/meter. $a=0.20$. 


\section{Barnacles}

Population Trend Power

$-10 \%$

$-5 \%$

$-3 \%$

$-2 \%$

$-1 \%$

$0 \%$

$1 \%$

$2 \%$

$3 \%$

$5 \%$

$10 \%$
1

0.934

0.6

0.406

0.268

0.212

0.244

0.402

0.602

0.886

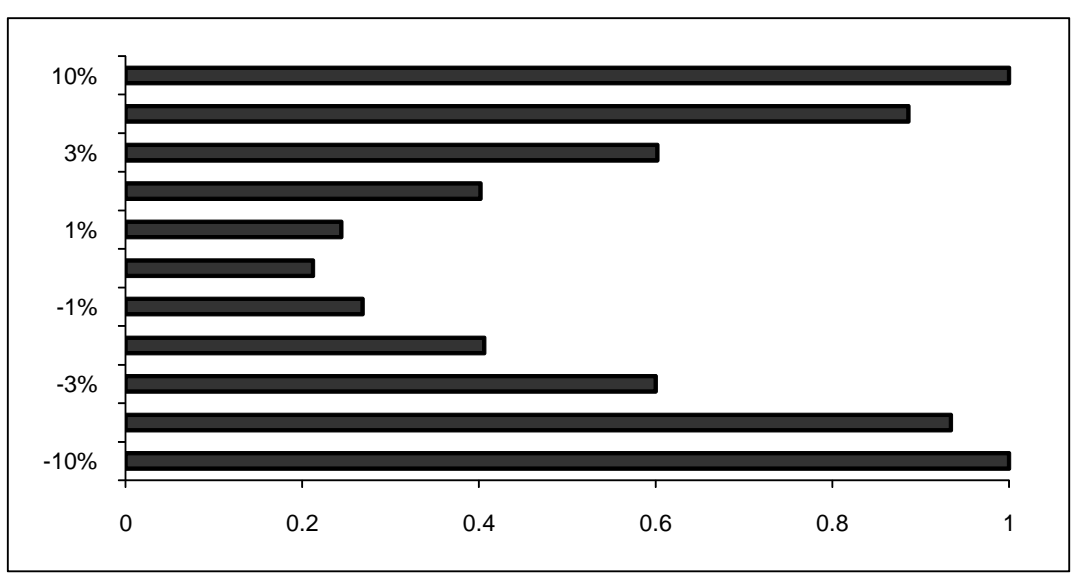

\section{Mytilus}

Population Trend Power

$$
-10 \%
$$

$-5 \%$

$-3 \%$

$-2 \%$

$-1 \%$

$0 \%$

$1 \%$

$2 \%$

$3 \%$

$5 \%$

$10 \%$

\section{Fucus}

Population Trend Power

$$
-10 \%
$$

$-5 \%$

0.652

$-3 \%$

0.43

$-2 \%$

0.242

$-1 \%$

0.202

$0 \%$

0.222

$1 \%$

0.22

$2 \%$

0.264

$3 \%$

0.422

$5 \%$

$10 \%$

0.654

0.98

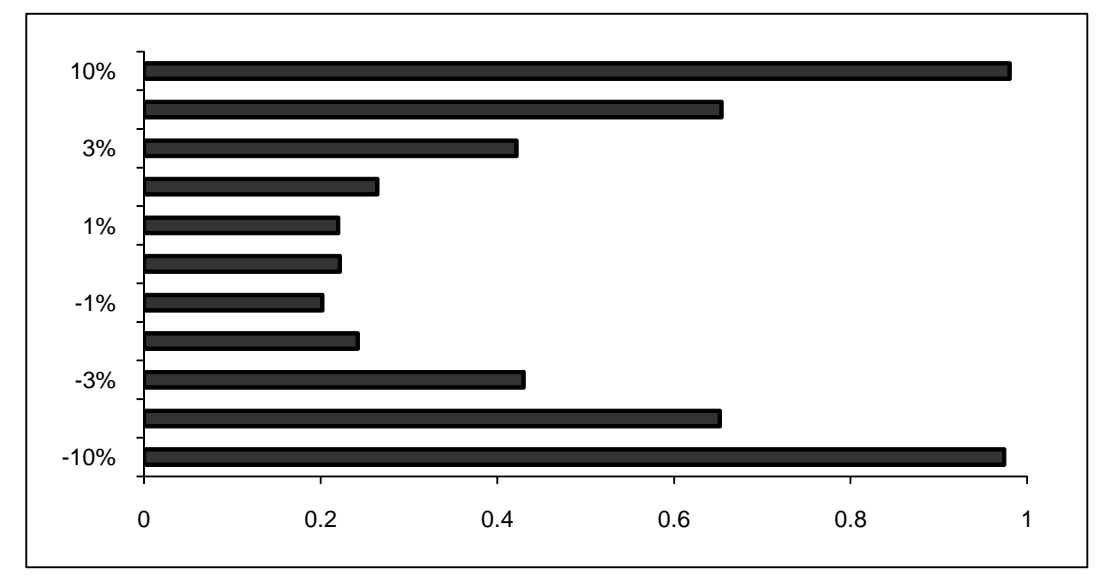

Figure C20. Power to detect population trends based on 3 years of data (1998-2001) in A. barnacles; B. Mytilus; and C. Fucus. Sampling was done on the level of 25 sites, 6 transects, 4 points/meter. $a=0.20$. 
Barnacles

\begin{tabular}{cc}
\hline Population Trend & Power \\
\hline$-10 \%$ & 1 \\
$-5 \%$ & 0.93 \\
$-3 \%$ & 0.668 \\
$-2 \%$ & 0.384 \\
$-1 \%$ & 0.252 \\
$-0 \%$ & 0.22 \\
$1 \%$ & 0.3 \\
$2 \%$ & 0.436 \\
$3 \%$ & 0.624 \\
$5 \%$ & 0.892 \\
$10 \%$ & 1
\end{tabular}

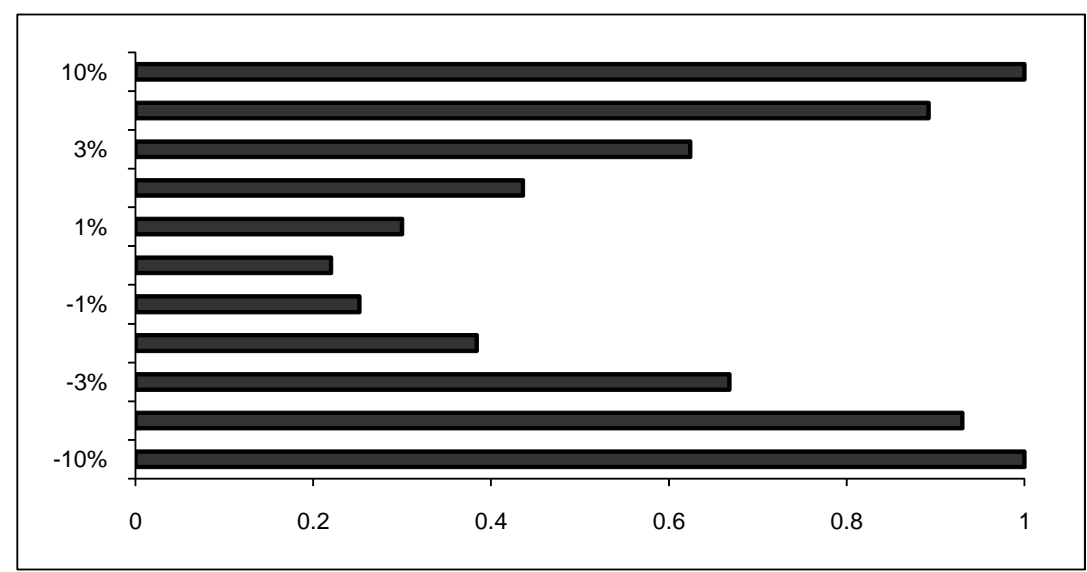

Mytilus

\begin{tabular}{cc}
\hline Population Trend & Power \\
\hline$-10 \%$ & 1 \\
$-5 \%$ & 0.928 \\
$-3 \%$ & 0.666 \\
$-2 \%$ & 0.484 \\
$-1 \%$ & 0.298 \\
$-0 \%$ & 0.196 \\
$1 \%$ & 0.32 \\
$2 \%$ & 0.456 \\
$3 \%$ & 0.69 \\
$5 \%$ & 0.92 \\
$10 \%$ & 1
\end{tabular}

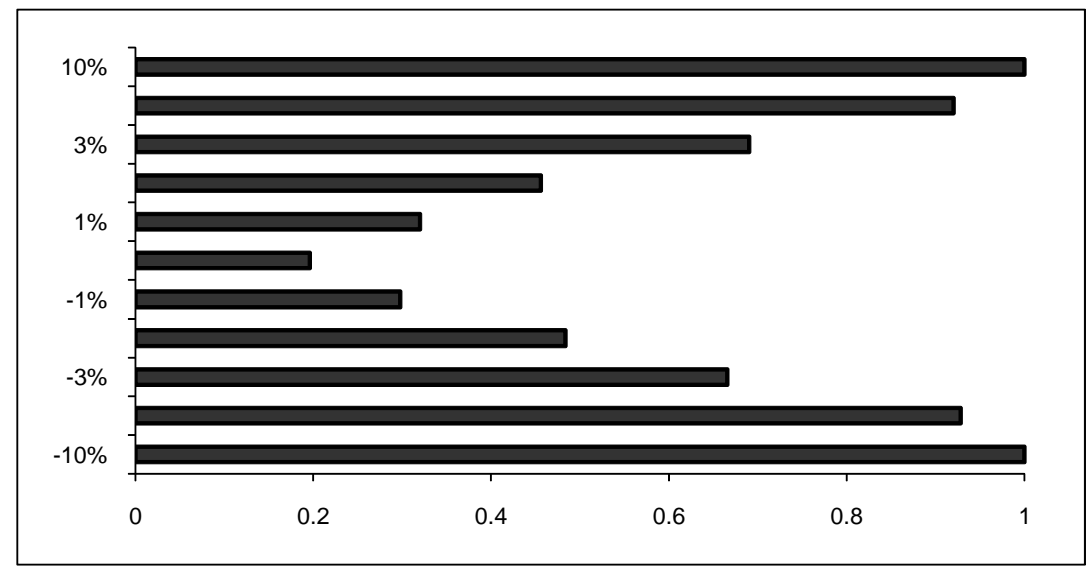

\section{Fucus}

\begin{tabular}{cc}
\hline Population Trend & Power \\
\hline$-10 \%$ & 0.984 \\
$-5 \%$ & 0.654 \\
$-3 \%$ & 0.366 \\
$-2 \%$ & 0.3 \\
$-1 \%$ & 0.262 \\
$-0 \%$ & 0.206 \\
$1 \%$ & 0.212 \\
$2 \%$ & 0.236 \\
$3 \%$ & 0.368 \\
$5 \%$ & 0.62 \\
$10 \%$ & 0.956
\end{tabular}

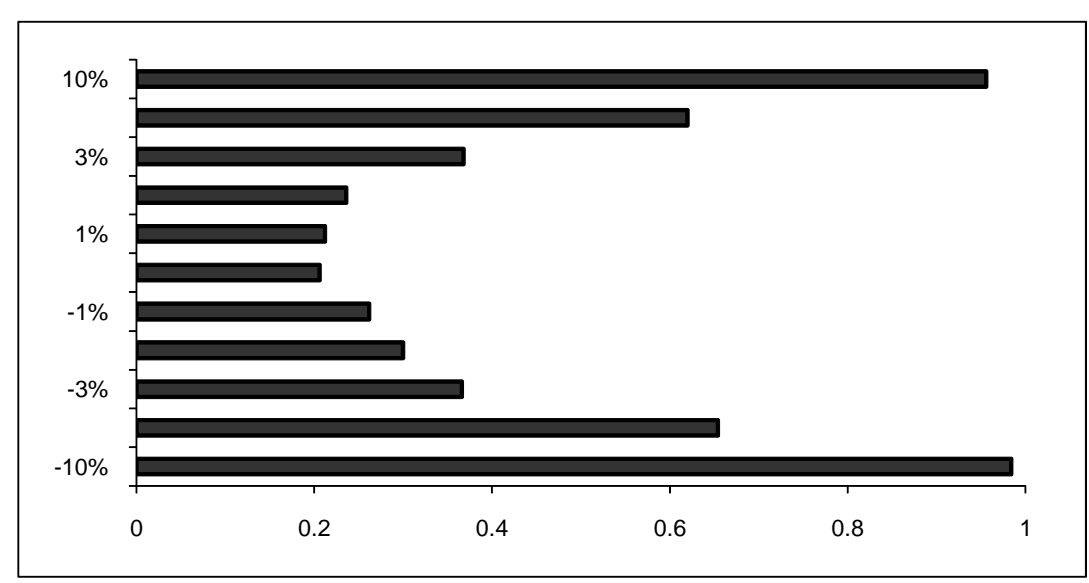

Figure C21 Power to detect population trends based on 3 years of data (1998-2001) in A. barnacles; B. Mytilus; and C. Fucus. Sampling was done on the level of 25 sites, 6 transects, 5 points/meter. $a=0.20$. 

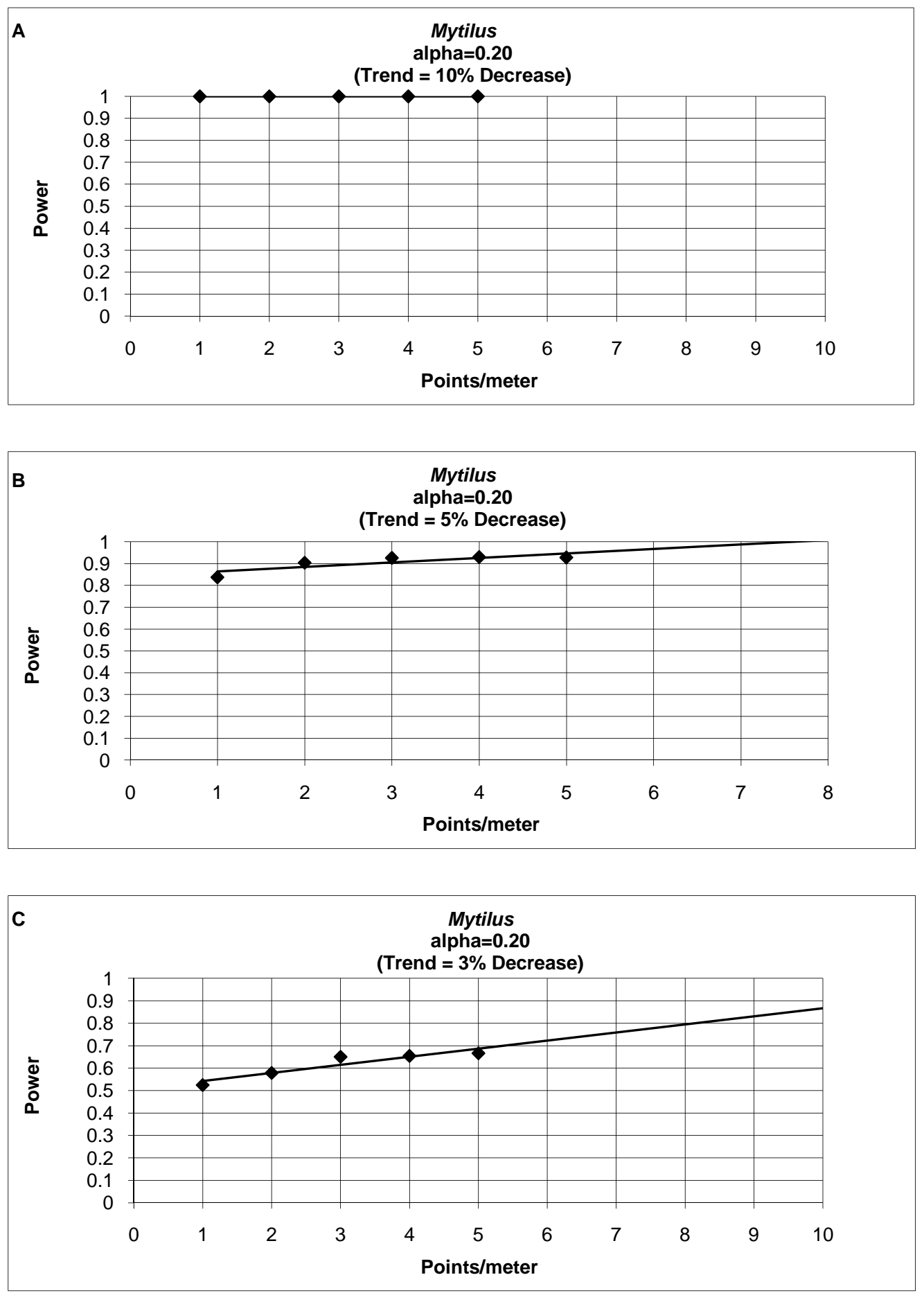

Figure C22. Power to detect a decline in Mytilus populations as a function of the number of points sampled per meter of each transect. Data are based on 3 years of sampling (1998-2001) at 25 sites, 6 transects at each site. $\alpha=0.20$. A. $10 \%$ decrease; B. $5 \%$ decrease; C. $3 \%$ decrease. Points on graphs indicate data; lines are linear projections of the trends suggested by these data. 


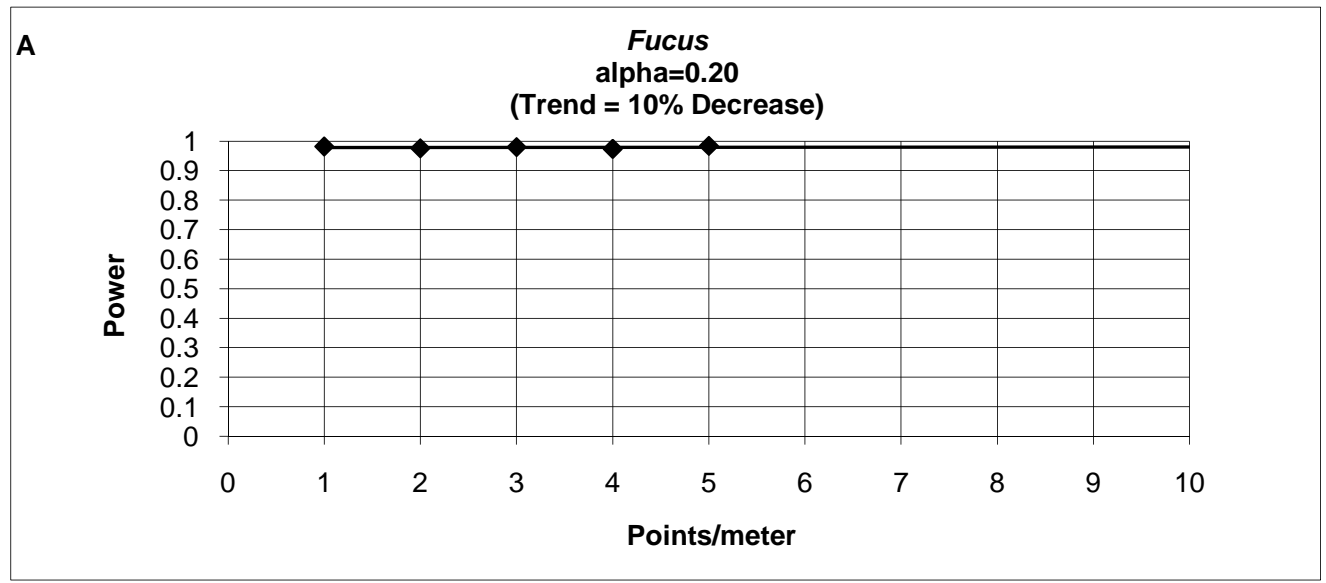

B

Fucus

alpha $=0.20$

(Trend = 5\% Decrease)
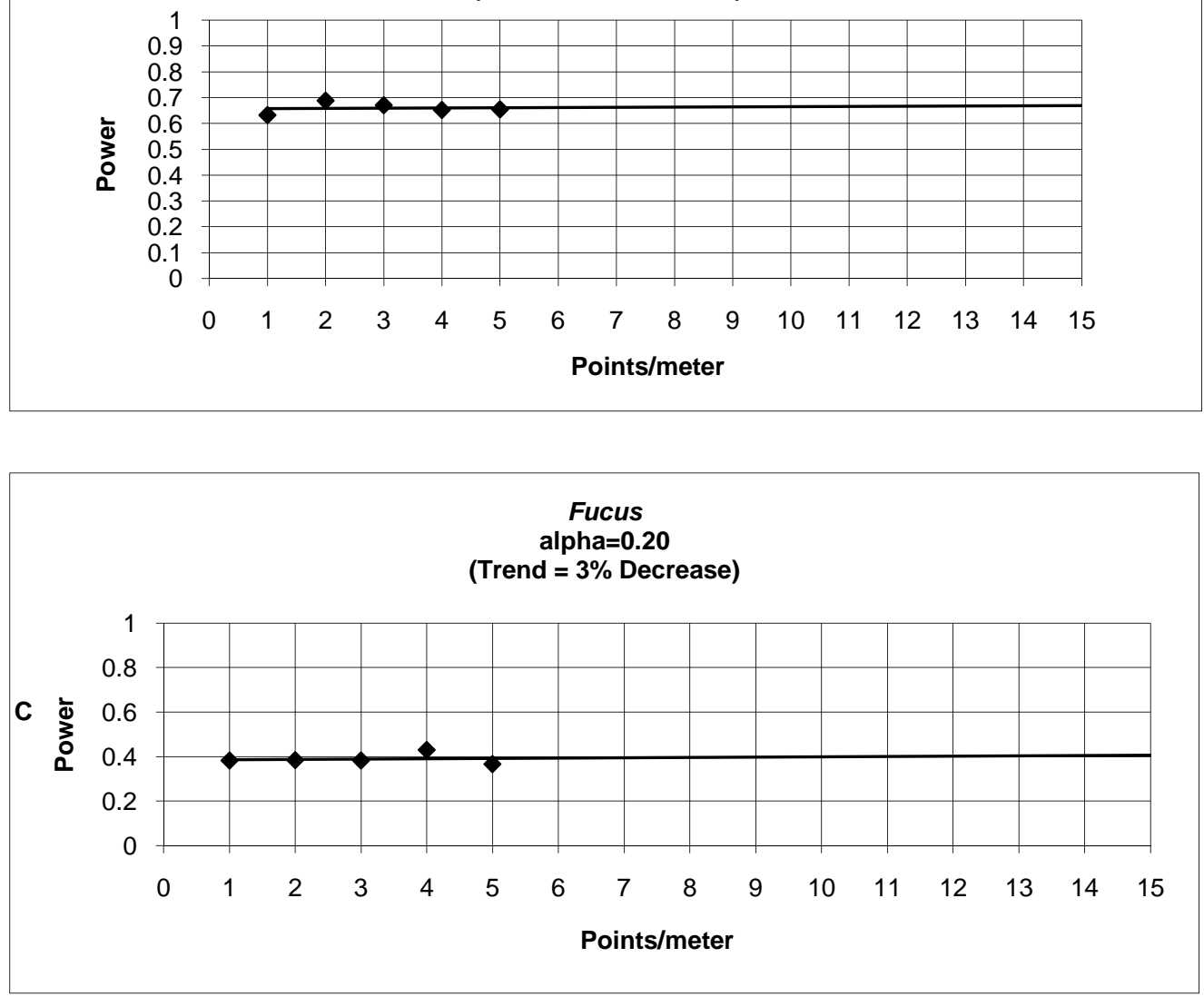

Figure C23. Power to detect a decline in Fucus populations as a function of the number of points sampled per meter of each transect. Data are based on 3 years of sampling (1998-2001) at 25 sites, 6 transects at each site. $a=0.20$. A. $10 \%$ decrease; B. $5 \%$ decrease; $C$. $3 \%$ decrease. Points on graphs indicate data; lines are linear projections of the trends suggested by these data. 


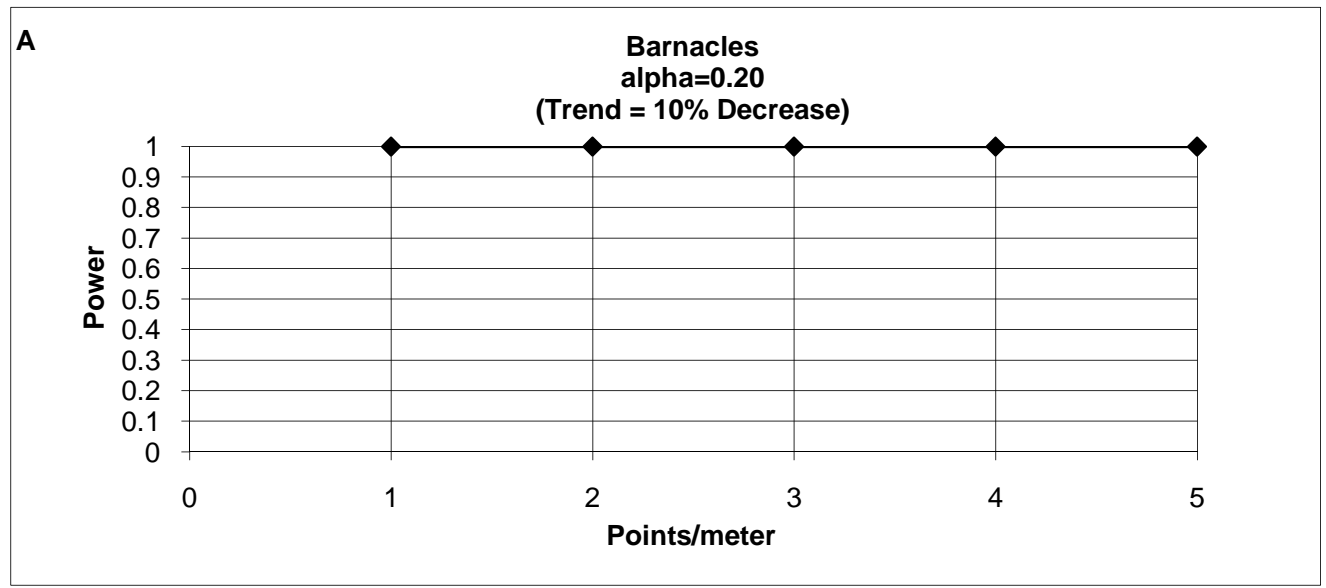

B

Barnacles

alpha $=0.20$

(Trend $=5 \%$ Decrease)
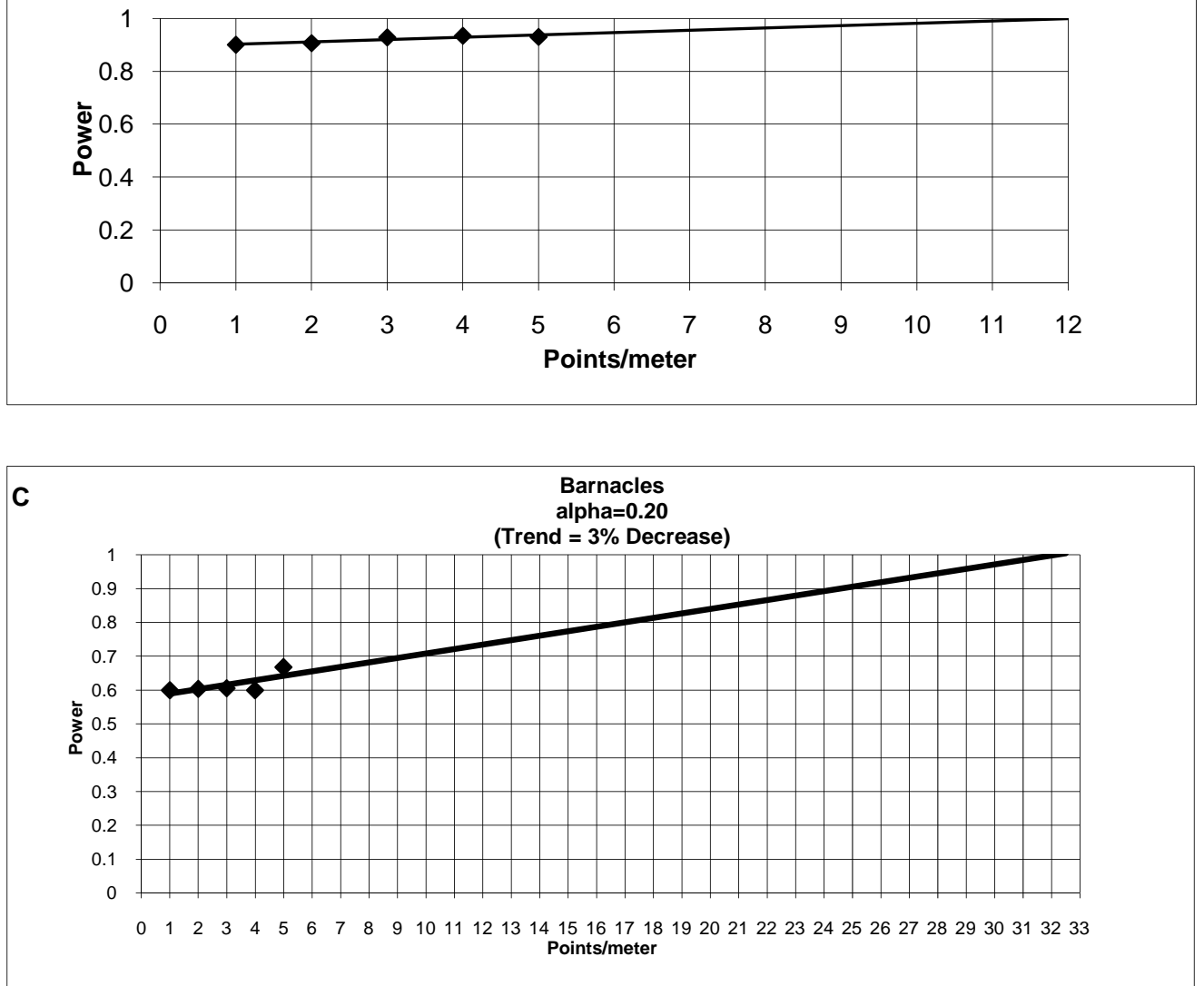

Figure C24. Power to detect a decline in barnacle populations as a function of the number of points sampled per meter of each transect. Data are based on 3 years of sampling (1998-2001) at 25 sites, 6 transects at each site. $a=0.20$. A. $10 \%$ decrease; B. $5 \%$ decrease; C. $3 \%$ decrease. Points on graphs indicate data; lines are linear projections of the trends suggested by these data. 

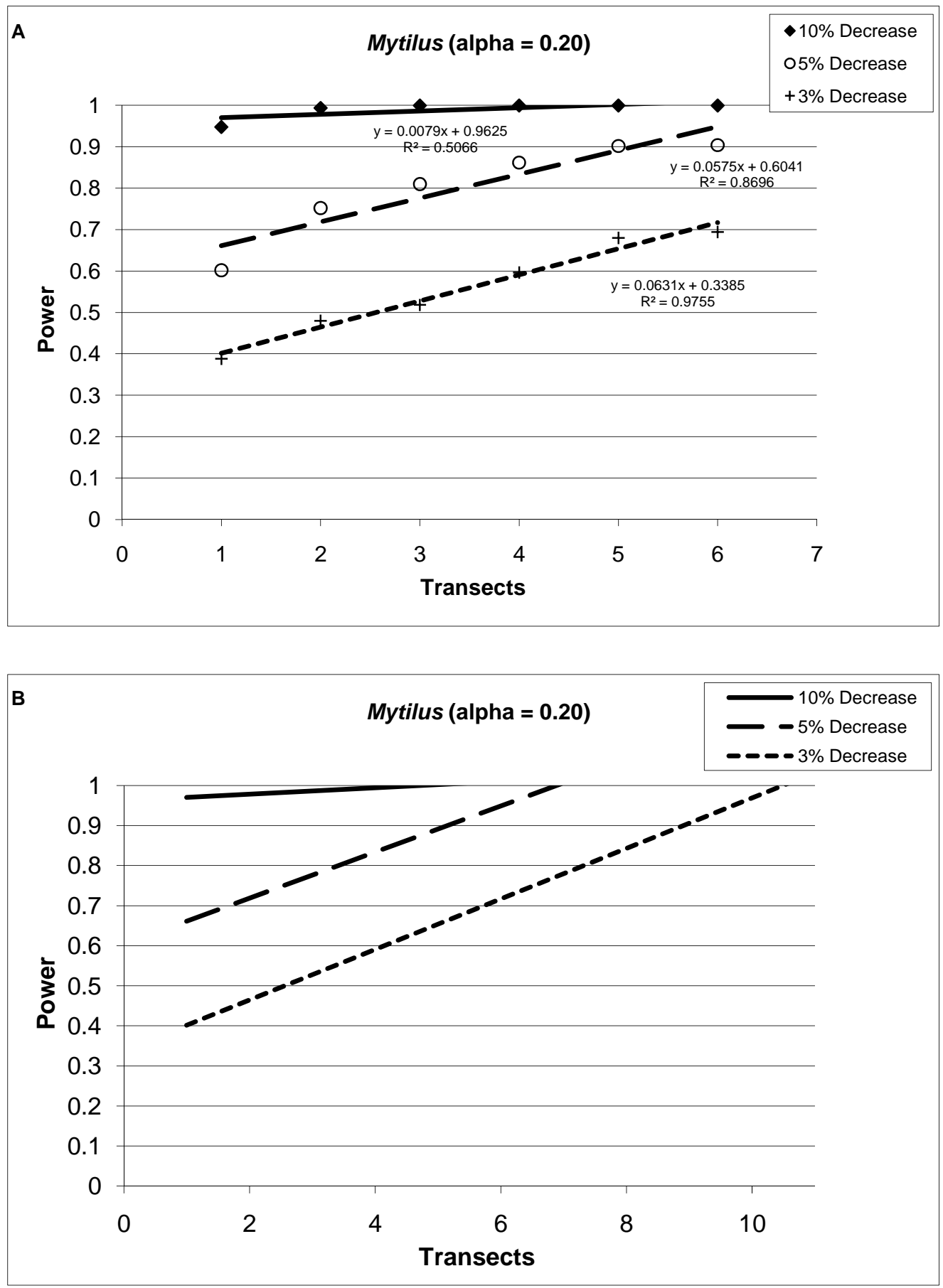

Figure C25. Power to detect declines in Mytilus populations (10\%, 5\%, and $3 \%)$ as a function of the number of transects sampled at each site. Transects were sampled at a frequency of 1 point/meter. Data are based on the analysis of 25 sites sampled over 4 years (1997-2001). $\alpha=0.20$. A. Points graphed using data. B. Linear trend of power based on data. 

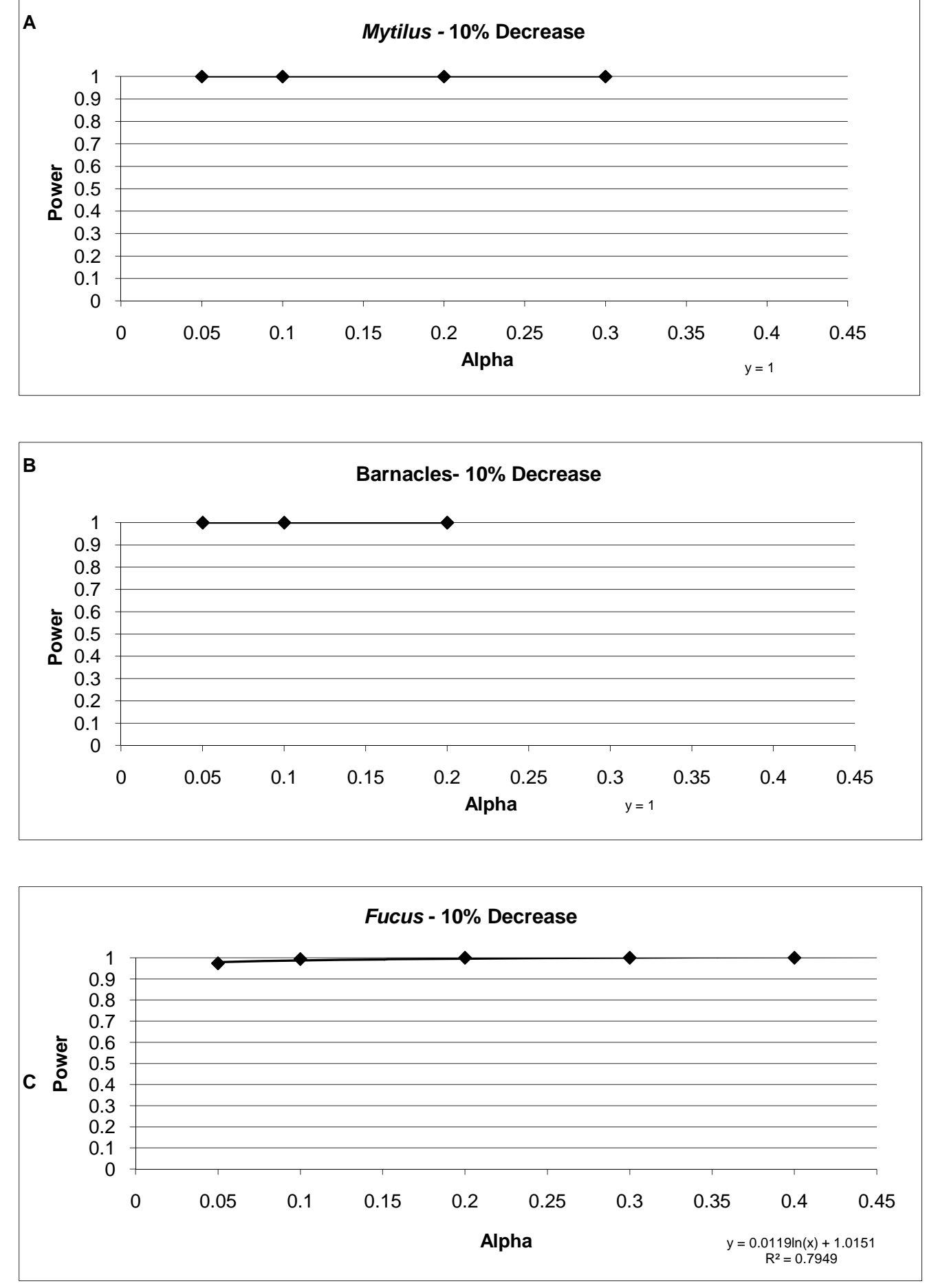

Figure C26. The relationship between alpha and the power to detect a $10 \%$ decline in the populations of the predominant species A. Mytilus, B. barnacles, C. Fucus. All data are based on 4 years of sampling (1997-2001) at the coarse-grained level: 25 sites, 6 transects, 1 point/meter. 


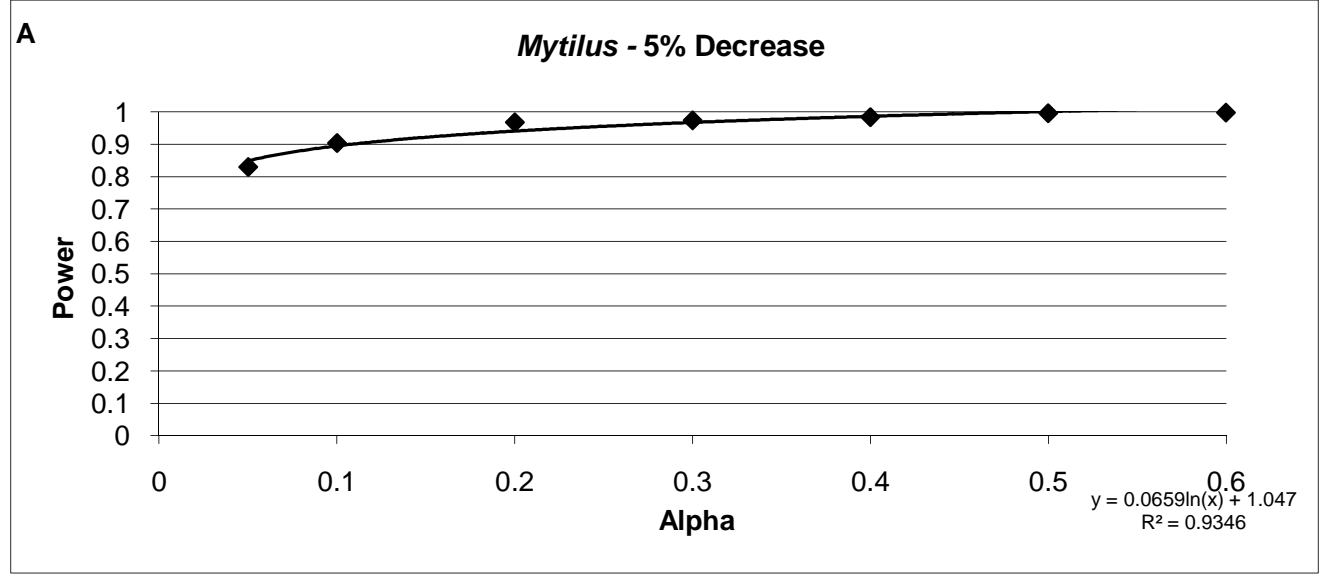

B Barnacles- 5\% Decrease
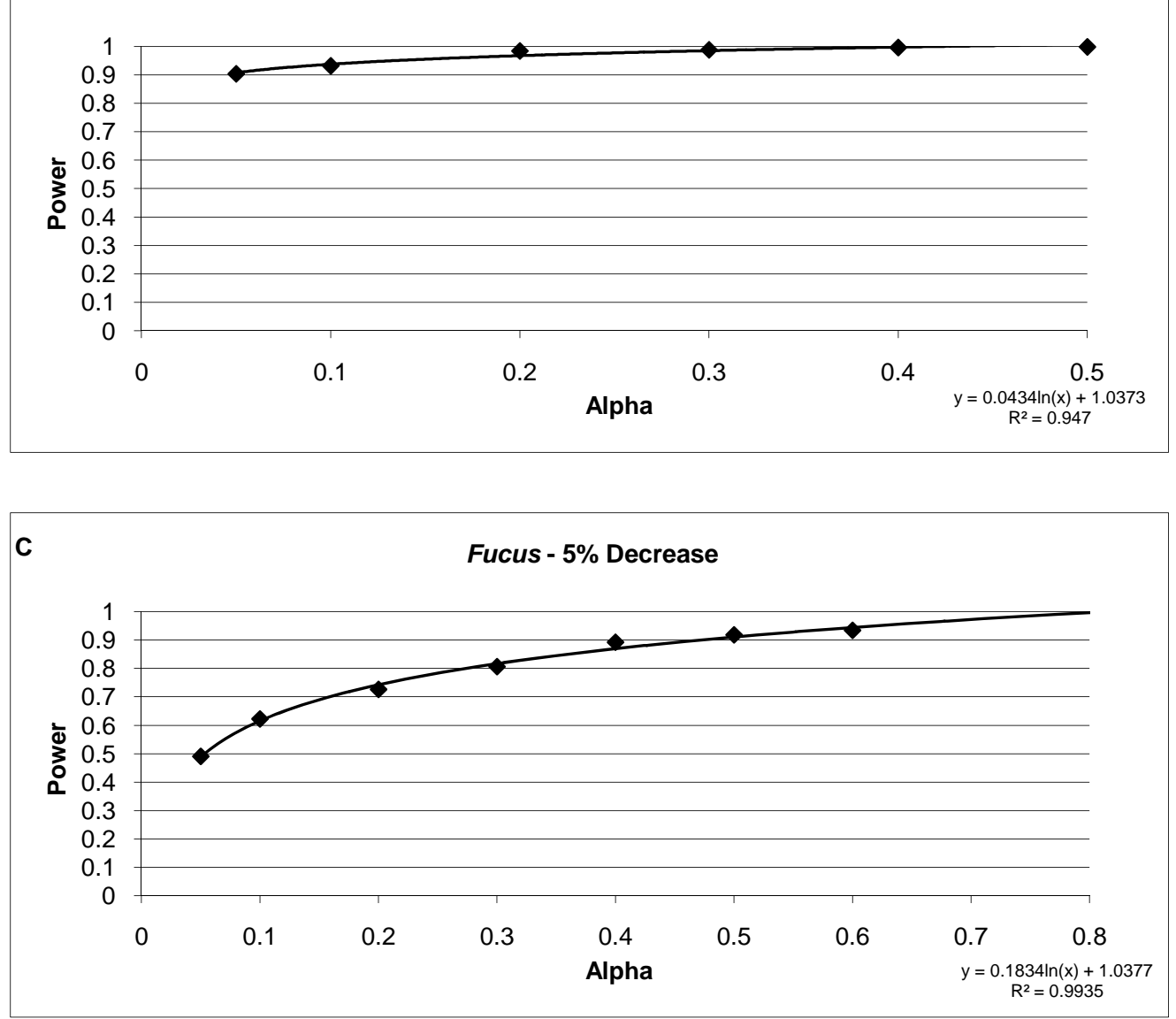

Figure C27. The relationship between alpha and the power to detect a $5 \%$ decline in the populations of the predominant species. A. Mytilus, B. barnacles, C. Fucus. All data are based on 4 years of sampling (19972001) at the coarse-grained level: 25 sites, 6 transects, 1 point/meter. 
This page left intentionally blank 


\title{
APPENDIX D - Standard Operating Procedures for Coarse-Grained Sampling
}

\author{
Standard Operating Procedures \\ for \\ Coarse-Grained Intertidal Monitoring Protocol
}

\section{Purpose}

Sampling at this coarse-grained level has as its purpose the broad-scale sampling of a number of sites that have been selected so as to be representative of a particular habitat type.

\section{Site Selection}

The selection of which particular segments to survey under the coarse-grained sampling level involved several steps: (1) the characterization of habitat types of an inferential set of segments within Glacier Bay proper (see aerial survey SOP), (2) selection of the habitat type for examination, and (3) selection of specific segments of the chosen habitat type.

\section{Characterization of habitat types with Glacier Bay proper}

Within Glacier Bay National Park and Preserve the coastline of Glacier Bay proper (the inner part of Glacier Bay NP\&P, from Pt. Carolus and Pt. Gustavus northward) was divided into $200 \mathrm{~m}$ segments using ArcInfo software and a digitized coastline (Geiselman, J. et. al., 1997). This resulted in the identification of 5,545 segments representing a coastline of 1,109 km. Based on our assessment that we could aerially survey 250 segments, a random number was used to identify a particular segment, and 250 segments were then systematically chosen relative to that initial randomly chosen start. Two hundred and forty-two of these segments were surveyed by fixed-wing airplane (see SOP for aerial surveys). The aerial surveys provided estimates of the relative abundance of different substrate types (bedrock, cobble/boulder, pebble/gravel, sand/silt/mud, coarse sand) within segments as well as biota (Fucus, mussels, barnacles). The survey results provided a characterization of the habitat types within Glacier Bay proper, and a pool of segments of different habitat types that had inference to the surveyed region.

2. Selection of the habitat type and the specific segments for examination We initially had been asked by the park to study protected rocky (bedrock) habitat. However, only 3 of the 241 (one of the 242 segments surveyed lacked slope data) segments surveyed by air were comprised of $76 \%$ or greater bedrock, with a slope of $<60$ degrees. We decided, on the basis of further discussions with Center staff and the Park staff, to relax the habitat parameters and include unconsolidated substrates (cobble/boulder) as well as bedrock sites as targets of this study. Thus the combined habitat type was defined as any segments having from $1-100 \%$ bedrock with slopes $<60$ degrees and/or $0 \%$ bedrock but $76-100 \%$ cobble/boulder and also a slope $<60$ degrees. This resulted in a pool of more than 100 potential coarse-grained sampling sites. Thirty sites were randomly chosen from this pool. Since the substrate percent coverages and slopes were estimates from the aerial surveys, an additional thirty sites were randomly chosen as extras in case closer inspection led to the elimination of many from the initial thirty. Elimination criteria included: inaccessibility because the site was too steep, or was a designated wildlife protection area; the presence of too much mud; lack of emergence during high tides (the site 
was not a true beach). Twenty-five of the original thirty selected segments were ultimately sampled (fig. 1).

\section{Within-Site Sampling:}

After (1) locating a site with GPS and photos, the within-site sampling involves: (2) establishment of the horizontal segment line at the approximate MHHW line, (3) establishing vertical transects, (4) point sampling of vertical transects, (5) sampling of bands that included each vertical transect, (6) photos, and (7) transect slope determinations.

\section{Site Location}

Navigate to the $200 \mathrm{~m}$ beach segment using the PLGR (Y-code data) GPS unit, aided by use of maps and photographs. The GPS coordinates indicate the location of the site's upper left corner (fig. D1).

One permanent marker, a 2-foot length of rebar, was placed on the left-hand side of the segment, above the site. The marker was placed perpendicular to the $200 \mathrm{~m}$ segment tape, upbeach enough to be well above the highest high tide and often into the terrestrial vegetation. A stainless steel 1 1/4" washer with "USGS/BRD, 1997 \#\#\#" stamped into it (\#\#\# is the segment number) was attached to the rebar by a cable tie. Orange flagging tape was tied around the rebar to make it visible in the photos and easier to relocate. The rebar stake was z-sparred (Splash Zone, a marine epoxy) in place if the substrate was suitable. Notes on the placement, including distance and compass bearings from the top left of the $200 \mathrm{~m}$ segment (the beginning of the horizontal segment line), were recorded in the field notes for the site.

At some sites, additional rebar were added above the horizontal segment line at $100 \mathrm{~m}$ and $200 \mathrm{~m}$. Also, for at least some of the six fine-grained sites sampled in 1997 (sites 36, 59, 63, 69, 89, and 217), there were rebar markers placed above each of the vertical transect lines. Under the fine-grained sampling there were 10 vertical transects sampled at each of these sites, each 20 meters apart, but with the first transect location determined randomly within the first 20 meters (e.g., systematic placement with a random start).

\section{Establish horizontal segment line}

The horizontal segment line is established at the approximate MHHW (mean higher high water) line by laying out $200 \mathrm{~m}$ of measuring tape, starting at the left upper edge of the segment and running across the beach. The segment line follows the lower Verrucaria (black lichen) zone (defined as approximately $20 \%$ coverage of Verrucaria), at the juncture of the Verrucaria and barnacle zones. Lay the tape parallel to the shoreline. If Verrucaria is not visible or is not continuous, estimate the highest tide line using cues of beach wrack and/or the beach grass line or above the upper barnacle zone. Follow the contours of the beach/shoreline. If you come to narrow but deep crevices or indentations, do not run the segment line up into the space, rather cut across it maintaining a line parallel with the shoreline. In some cases littorine snails will be found above the horizontal segment line. Note the distance from the site-marking rebar (above the left edge of the segment) to the horizontal segment line (MHHW). If $100 \mathrm{~m}$ and $200 \mathrm{~m}$ rebar markers are present, also note the distances from those to the horizontal segment line.

\section{Establish Vertical Transects}

Six line transects ("vertical" transects) are positioned parallel to the elevational gradient of the segment, running from the horizontal segment line down to MLLW (mean lower low water, the $0-\mathrm{m}[=0$ - $\mathrm{ft}$ t tide level). The start of the first vertical transect is randomly chosen while the remaining transects are placed systematically every $33 \mathrm{~m}$ relative to the location of the first. Prior to sampling, establish a list of 25 
random numbers between 0 and 33 to be used to establish the location of the first vertical transect for each of the 25 coarse-grained sites. The random number indicates the distance along the horizontal segment tape to the start of the first transect. For example, if the random number for a site is 12 , the first vertical transect is located $12 \mathrm{~m}$ from the left edge of the segment. Succeeding transects are set every 33 $\mathrm{m}$ (at 45, 78, 111, 144, and $177 \mathrm{~m}$ from the left end of the $200 \mathrm{~m}$ segment tape, when the random number is 12). If a vertical transect falls on a section of the segment that is unsampleable due to steep slope or fresh water input, then determine the horizontal distance of the unsampleable area and shift the remaining transects that distance to the right (fig. D2). Vertical transects are run perpendicular to the shoreline. On concave beaches, positioning the transects perpendicular to the shoreline keeps them from overlapping (fig. D3). Natural variation in the complexity of the topography necessitates some decisionmaking in the layout of the transect lines. For example, when large rocky outcrops too high for sampling intervened, transect lines were interrupted (Site 108), with the rocky outcrop declared unsampleable, and the transect resumed on the shore-side of the rocky outcrop. The standard should be transect lines laid perpendicular to the shoreline. Transect lines are draped so as to generally conform to the substrate as they are laid, rather than being stretched taut. The intention is that all exposed surfaces have an equal probability of being sampled. The lowest extent of the transect, the 0 -m tide level, is determined by using the Tides and Currents computer program (Nautical Software, Inc.) for the sampling date and nearest tide station location (Bartlett Cove, Willoughby Island, Composite Island, or Muir Inlet). At the time of the 0-m tide (MLLW), other activities are stopped temporarily and all transect tapes are run to the water line, with the tape looped to indicate the 0-m location.

\section{Point sampling of Vertical Transects}

Selected points along the vertical transects are sampled to provide estimates of the relative percent coverage of biota along the transects, and across the site, as well as the relative percent coverage of underlying substrates. The sampling of points along these transects also gives a record of zonation of species. The density of sampling is five points sampled per meter, sampled each $20 \mathrm{~cm}$ (e.g., $0,0.2$, $0.4 \mathrm{~m}$, etc.). Tapes should be laid with the zero meter mark of the tape at the horizontal segment tape.

Points are sampled by noting the species and substrate at each point, using the right edge of the tape and the distance hash marks along the tape as cross hairs to mark the point. Use a pencil, knitting needle or other pointer to follow the point perpendicularly from the tape to the substrate. Sampling is 3-dimensional. Record all species underlying the point, including multiple "hits" or layers of the same species, then the substrate. Dense Fucus and mussels can be difficult to assess accurately in three-dimensional sampling (sampling probably tends to under-estimate the layering). Organisms are identified to the lowest taxonomic level possible in field sampling. Some taxa are grouped because they cannot be discriminated in the field readily (appendix D1.1). Use the abbreviations from the species list when recording. Substrates are classified according to a modified Wentworth-scale (appendix D1.2). The format for recording data is as follows: species/substrate (e.g. FU/BR =Fucus over Bedrock). If there is more than one species when following the point down to the substrate (e.g. littorine [Littorina sitkana] on a piece of Fucus which covers a mussel) then use the following format: (species, species, species.....)/substrate (e.g. (LSI,FU,MY)/BR). Multiple sequential layers or hits of the same species can be indicated by superscripts, e.g., $\mathrm{FU}^{3}$. If there is silt on another substrate, record the underlying substrate. An example of a completed data sheet is included in appendix D2. 


\section{Band Sampling}

The purpose of sampling a wider swath or band around each vertical transect, is to sample the density of larger or more mobile invertebrates that are less likely to be well-sampled by the points. After the points have been sampled on a vertical transect, band swaths are done. Scan the swath $1 \mathrm{~m}$ to each side of the transect for selected large or mobile species (starfish, sea urchins, or large chitons). Count the number of each band species observed per meter and record the number and location in the appropriate place on the data sheet. If no band species are observed, be sure to note that none were seen.

\section{Transect Slope Descriptions}

Take the slope of each vertical transect line, using a hand-held clinometer (e.g., Suunto clinometer). If there appears to be more than one distinct slope (e.g., the slope changes significantly along the transect line), take readings for each section, but also get an overall slope for the transect line by taking a measurement from the bottom to the top or vice versa. Also take the compass bearing of each transect line. Note if the compass bearing is from the bottom to the top or vice versa. Also note if magnetic or true north was used (thus far we have only used magnetic north).

\section{Photos}

The purpose of taking photographs is to record locations of transect lines, and to obtain a visual record of the geomorphology and biology, both along transect lines and across the site from several spots. Both slide and print film originally were used; these will be supplanted by digital photography. Prints provide easily used visual records and aid relocating the site marker and transect locations. Slide film was used for longer term records of transect shots and general site photos and for use in talks. Photos are taken from the bottom of each transect looking towards the top, from the top of each transect looking down, from the top left area looking towards the top right, and top right towards top left, from the bottom left looking towards the bottom right, bottom right to left, and especially from approximately midbeach between transects 3 and 4 looking right and left, from the top left towards the permanent marker or from the bottom left towards the permanent marker, and of anything that is of particular interest. Photos of the vertical transects from the top looking down were often taken, but not routinely. All photos are recorded in the field notes or on transect data sheets and later entered into a photolog.

8. Data entry and the Access database

These topics are discussed in appendix E.

\section{Reference}

Geiselman, J., Dunlap, J., Hooge, P., and D. Albert. (Eds). 1997. Glacier Bay Ecosystem GIS CD-ROM set. U.S. Geological Survey and Interrain Pacific. Anchorage and Juneau, Alaska. 


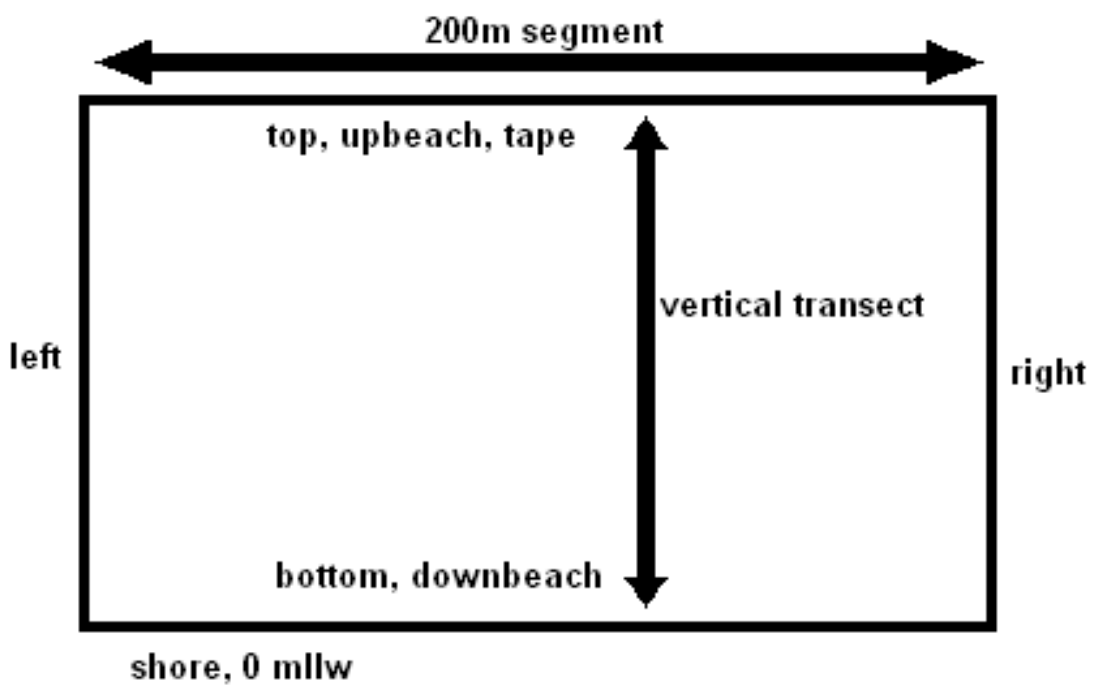

Figure D1. Directional terms used in the coastal monitoring protocol development study. 


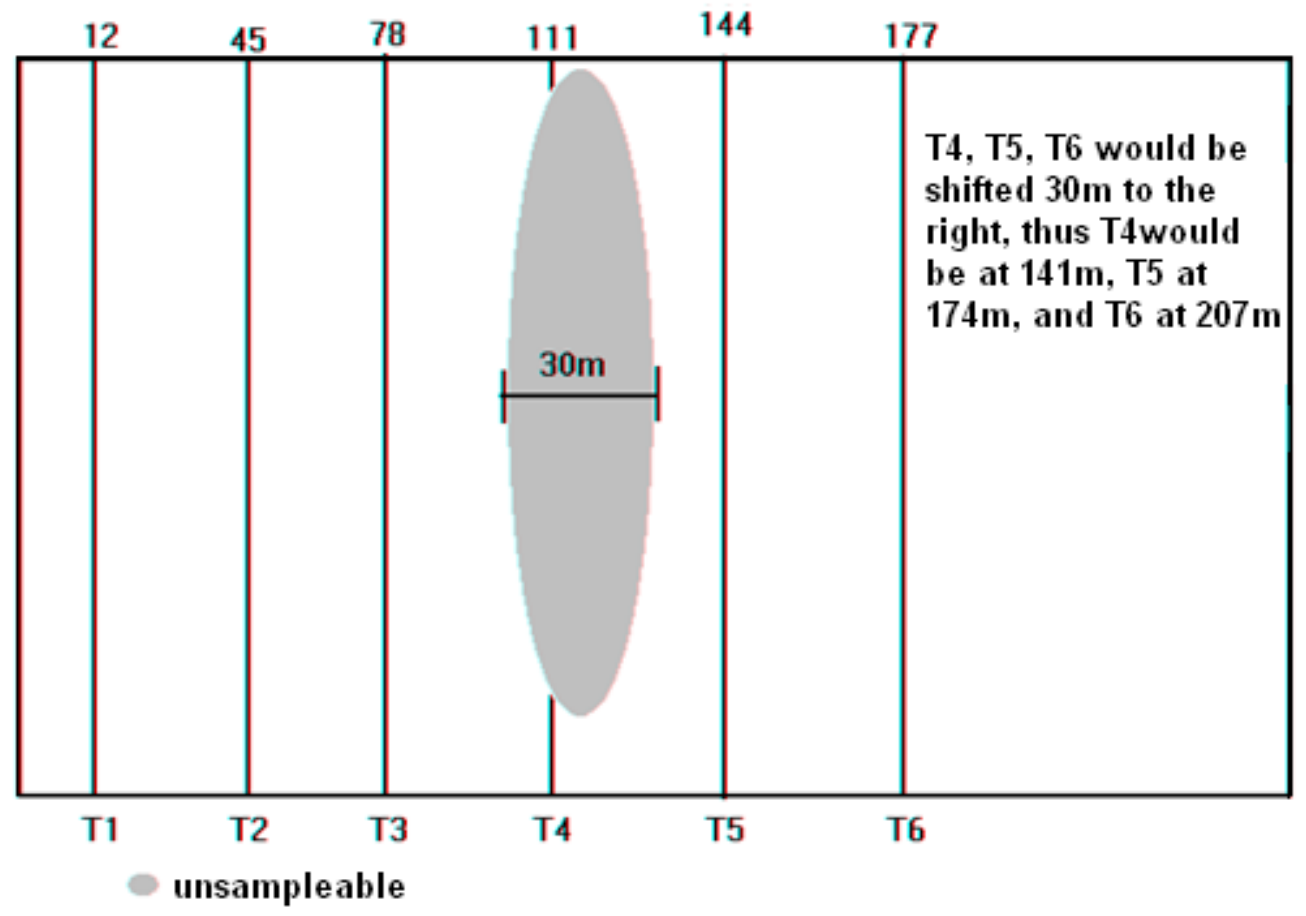

Figure D2. A rough depiction of the arrangement of vertical transects and how to deal with unsampleable areas. 


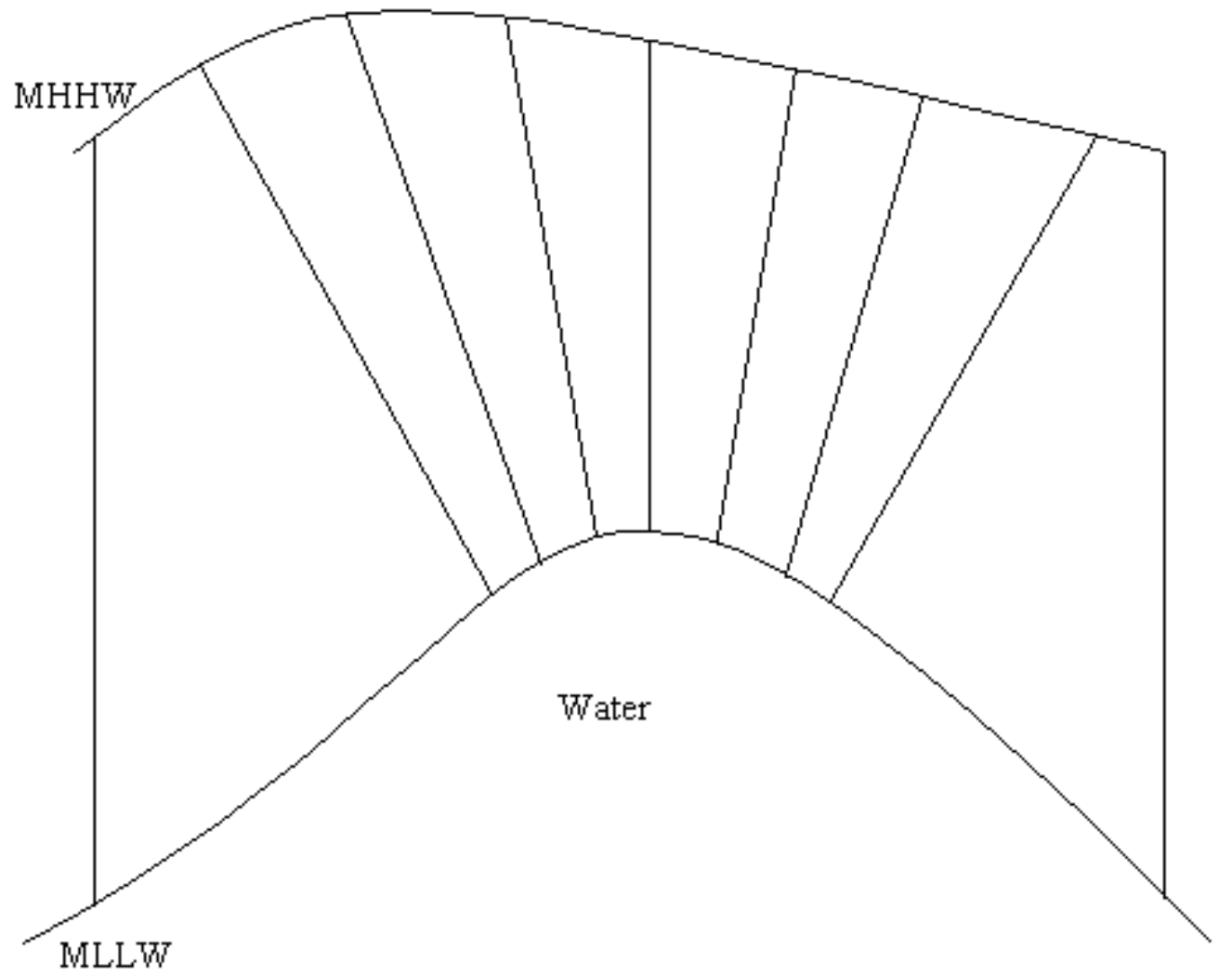

Figure D3. A schematic of vertical transects laid out at a concave beach. Note that the transect lines are laid perpendicular to the shoreline. 


\section{Appendix D1}

D1.1. List of species, substrates, and abbreviations used in the 1997 coastal monitoring protocol development project. Species noted during band swath searches are marked with an asterisk.

D1.2. The modified Wentworth scale used to classify substrates. This is taken from the sediment surface mapping protocol for Glacier Bay coastal mapping, coastal monitoring, and benthic surveys SOP, sedprot.w51. 
D1.1. Species and substrate abbreviations used in the 1997 coarse-grained sampling.

* = BAND SPECIES

\begin{tabular}{|c|c|c|c|c|c|}
\hline \multirow[t]{5}{*}{ MUSSELS } & & & RED ALGAE & & \\
\hline & Modiolus modiolus & MO & & articulated coralline algae & AR \\
\hline & Musculus spp. & MUS & & Cryptosyphonia woodii & CRY \\
\hline & Mytilus trossulus & MY & & Encrusting coralline algae & EC \\
\hline & & & & Endocladia muricata & ED \\
\hline \multirow[t]{3}{*}{ CLAMS } & & & & Gigartinaceae & GG \\
\hline & Hiatella arctica & $\mathrm{HI}$ & & Gloiopeltis furcata & GL \\
\hline & & & & Halosaccion glandiforme & $\mathrm{HA}$ \\
\hline \multirow[t]{7}{*}{ BARNACLES } & & & & Mastocarpus papillatus & MA \\
\hline & Balanomorpha & BA & & Neorhodamela/Odonthalia spp. & NEO \\
\hline & Balanus glandula/Semibalanus balanoides & BG & & Palmaria callophylloides & PC \\
\hline & Chthamalus dalli & $C D$ & & Palmaria spp. & PA \\
\hline & Semibalanus cariosus & SC & & Polysiphonia/Pterosiphonia spp. & PS \\
\hline & Barnacle spat/cyprids $<2 \mathrm{~mm}$ & BS & & Porphyra spp. & PR \\
\hline & & & & Red algal crust- fleshy & RE \\
\hline \multirow[t]{10}{*}{ LIMPETS } & & & & Small filamentous red & FIR \\
\hline & Acmaea mitra & ACM & & Small foliose red & FOR \\
\hline & Diadora spp. & DI & & & \\
\hline & Lottia digitalis & LD & BROWN ALGAE & & \\
\hline & Lottia pelta & LP & & Agarum clathratum & AG \\
\hline & Lottidae $<8 \mathrm{~mm}$ or questionable & LO & & Alaria fistulosa & AF \\
\hline & Tectura fenestrata & TF & & Alaria marginata (or ALM) & $A L$ \\
\hline & Tectura persona & TP & & Coilodesme spp. & $\mathrm{CL}$ \\
\hline & Tectura scutum & TES & & Costaria costata & $\cos$ \\
\hline & & & & Cymathere triplicata & $\mathrm{CY}$ \\
\hline \multirow[t]{17}{*}{ SNAILS } & & & & Desmarestia spp. & DE \\
\hline & Buccinum spp. & $\mathrm{BU}$ & & Fine filamentous brown & FIB \\
\hline & Fusitriton oregonensis & FUS & & Fucus gardneri & FU \\
\hline & Lacuna spp. & LA & & Laminaria bongardiana & LB \\
\hline & Littorina scutulata & LSC & & Laminaria saccharina & LS \\
\hline & Littorina sitkana & LSI & & Leathesia difformis & LEA \\
\hline & Littorina spp. & LIT & & Petalonia fascia & PET \\
\hline & Margarites spp. & MAR & & Scytosiphon/Melanosiphon spp. & SM \\
\hline & Neptunea spp. & NEP & & Small foliose brown & FOB \\
\hline & Nucella canaliculata & $\mathrm{NC}$ & & Soranthera ulvoidea & SOR \\
\hline & Nucella emarginata & NUE & & Thick brown crust & TBC \\
\hline & Nucella lamellosa & NLA & & & \\
\hline & Nucella lima & $\mathrm{NLI}$ & GREEN ALGAE & & \\
\hline & Onchidella borealis & ON & & Acrosiphonia spp. & $A C$ \\
\hline & Searlesia dira & SE & & Enteromorpha intestinalis & EN \\
\hline & Siphonaria thersites & SI & & Prasiola meridionalis & PM \\
\hline & & & & Small filamentous green & FIG \\
\hline \multirow[t]{6}{*}{ CHITONS* } & & & & Ulothrix spp. & Uo \\
\hline & Cryptochiton stelleri & CR & & Ulvales & UV \\
\hline & Katharina tunicata & KA & & & \\
\hline & Mopalia spp. & MOP & SEAGRASS & & \\
\hline & Tonicella spp. & TO & & Phyllospadix serrulatus & $\mathrm{PHY}$ \\
\hline & & & & Zostera marina & ZO \\
\hline
\end{tabular}




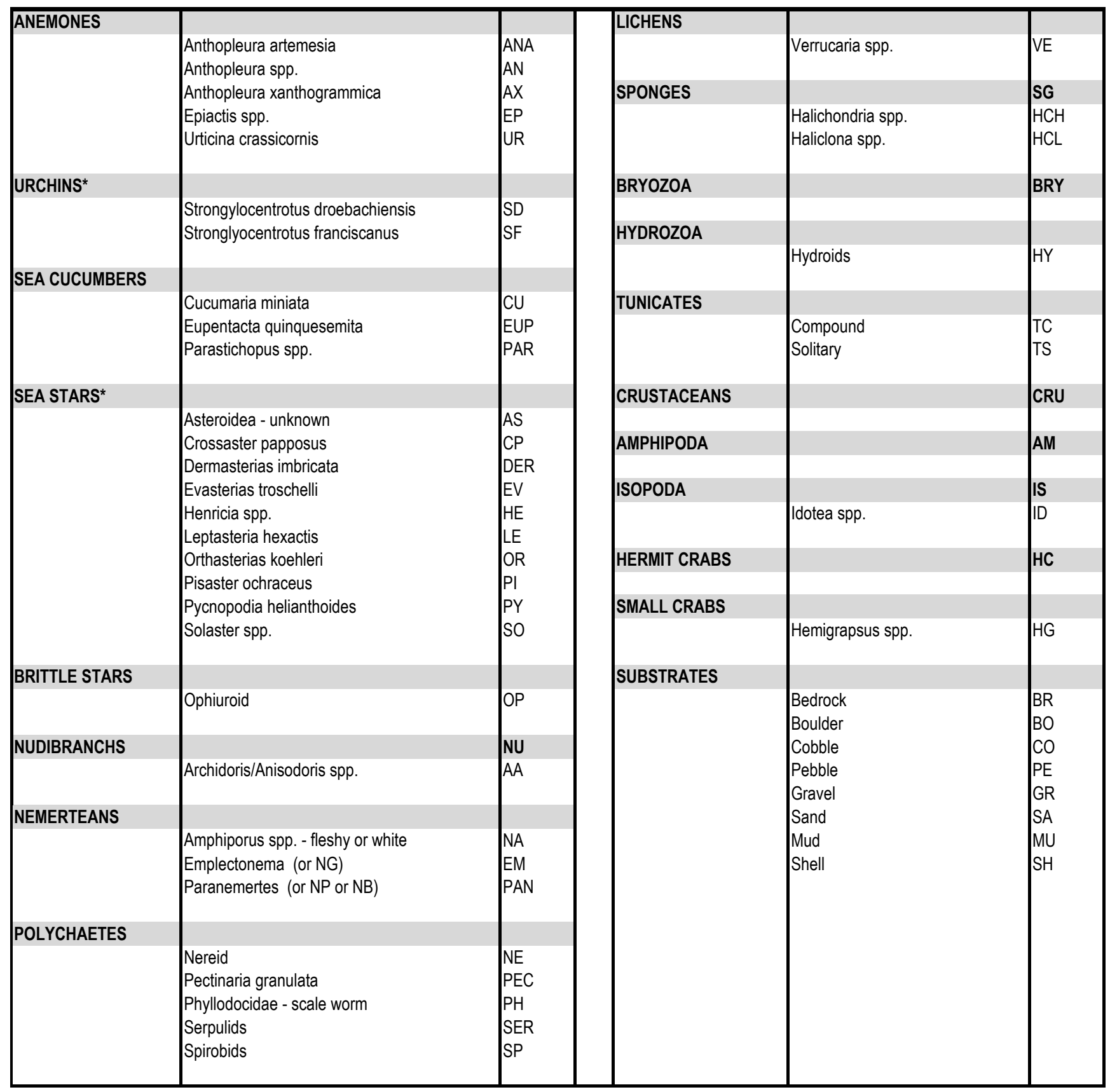


D1.2. Substrate categories used in the 1997 coarse-grained sampling.

\begin{tabular}{|l|l|l|}
\hline Substrate & Wentworth Size & Description \\
\hline bedrock & continuous rock surface & NA \\
\hline boulder & $>256 \mathrm{~mm}$ diameter & head size or larger \\
\hline cobble & $64-256 \mathrm{~mm}$ diameter & billiard ball size to head size \\
\hline pebble & $4-64 \mathrm{~mm}$ diameter & pea size to billiard ball size \\
\hline gravel & $2-4 \mathrm{~mm}$ diameter & BB size to pea size \\
\hline sand & $1 / 16-2 \mathrm{~mm}$ diameter & just gritty in fingers to pin head size \\
\hline mud/silt & NA & floury coating, not gritty, similar to clay \\
\hline shell & NA & whole shell or identifiable fragments \\
\hline
\end{tabular}




\section{Appendix D2}

D2.1. An example of a completed data sheet from a vertical transect sampled during the 1999 coarse-grained sampling.

\begin{tabular}{|c|c|c|c|c|c|c|c|}
\hline \multirow{2}{*}{\multicolumn{2}{|c|}{$\begin{array}{l}\text { SITE \# } 200 \\
\text { DATE } 14 \text { lume } 99 \\
\text { TRANSECT\# } 5\end{array}$}} & \multirow{2}{*}{\multicolumn{4}{|c|}{ OBSERVER $\mathrm{Jenn} / \mathrm{deff}$}} & \multirow{2}{*}{\multicolumn{2}{|c|}{$\begin{array}{l}\text { PAGE\# } 1 \text { of } \frac{1}{1} \text { - COMPASS HEAD: } \\
\text { - }\end{array}$}} \\
\hline & & & & & & & \\
\hline $\begin{array}{r}\text { POINT } \\
0.0 \\
\end{array}$ & Bिण BRPISUBSTRATE & $\begin{array}{c}\text { BAND SPP \& \# } \\
0 \\
\end{array}$ & $\begin{array}{c}\text { SLOPE } \\
18^{\circ}\end{array}$ & \begin{tabular}{||r|} 
POINT \\
8.4 \\
\end{tabular} & $\begin{array}{l}\text { SPP/SUBSTRATE } \\
M Y^{2} / B O\end{array}$ & $\begin{array}{c}\text { BAND SPP \& \# } \\
0\end{array}$ & $\mid \begin{array}{r}\text { SLOPE } \\
10^{\circ}\end{array}$ \\
\hline 0.2 & Bo & & & 8.6 & $\mathrm{BO}$ & & \\
\hline 0.4 & 60 & & & 8.8 & MY /BO & & \\
\hline 0.6 & BO & & & 9.0 & Bo & & \\
\hline 0.8 & (20) & & & 9.2 & \begin{tabular}{|l|l|} 
MY & BO \\
\end{tabular} & & \\
\hline 1.0 & 80 & & & 9.4 & $\mathrm{MY} / \mathrm{BO}$ & & \\
\hline 1.2 & $\mathrm{BO}$ & & & 9.6 & BO & & \\
\hline 1.4 & 362 & & & 9.8 & FU 1 BO & & \\
\hline 1.6 & 30 & & & 10.0 & $B O$ & & \\
\hline 1.8 & 30 BR & 7 & & 10.2 & $M Y / B O$ & & \\
\hline 2.0 & FIA $\mathrm{BG} \mid \mathrm{BO}$ & & & 10.4 & $P R^{2} \mid B O$ & 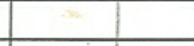 & \\
\hline 2.2 & $\mathrm{FU}^{2} \mathrm{BG} / \mathrm{BO}$ & & & 10.6 & $M Y Z / B O$ & & \\
\hline 2.4 & $\angle S 1, B G / B O$ & & & 10.8 & $B O$ & & \\
\hline 2.6 & $\mathrm{BG} / \mathrm{BO}$ & & & 11.0 & $B O$ & & \\
\hline 2.8 & $F u^{2} B G \mid B R$ & & & 11.2 & $M Y / B O$ & 1 & 1 \\
\hline 3.0 & $F U L S I I B R$ & & & 11.4 & & & \\
\hline 3.2 & $F_{1}^{6} B G / B O$ & & & 11.6 & & & \\
\hline 3.4 & $\mathrm{FU}^{3} \mathrm{BG} \mid \mathrm{BO}$ & & & 11.8 & & & \\
\hline 3.6 & Fu $1 B O$ & & & 12.0 & & & \\
\hline 3.8 & $B G / B O$ & & & 12.2 & & & \\
\hline 4.0 & $\mathrm{FU}^{5} \mathrm{BG} / \mathrm{BO}$ & & & 12.4 & & & \\
\hline 4.2 & $\mathrm{FU}^{5} \mathrm{BG} / \mathrm{BO}$ & & & \begin{tabular}{|l|}
12.6 \\
\end{tabular} & & & \\
\hline 4.4 & $\mathrm{Fu}^{2} / \mathrm{BO}$ & & & \begin{tabular}{|l|}
12.8 \\
\end{tabular} & & & \\
\hline 4.6 & $\mathrm{Fu}^{3} / \mathrm{BO}$ & & & 13.0 & & & \\
\hline 4.8 & $\mathrm{Fu}^{4} \mathrm{MY} / \mathrm{BO}$ & & & \begin{tabular}{|l|}
13.2 \\
\end{tabular} & & & \\
\hline 5.0 & $\mathrm{Fu}^{3} \mathrm{MY} \mathrm{Fu}^{2} \mathrm{MY} / \mathrm{BO}$ & & & \begin{tabular}{|l|}
13.4 \\
\end{tabular} & & & \\
\hline 5.2 & MY FIG $\mid B O$ & & & 13.6 & & & \\
\hline 5.4 & $F U, M Y F U, F I G, B G / B$ & & $\perp$ & 13.8 & & & \\
\hline 5.6 & FIG AC MY BG/BO & & $50^{\circ}$ & \begin{tabular}{|l|}
14.0 \\
\end{tabular} & & & \\
\hline 5.8 & $B G M Y^{2} / B O$ & & 1 & \begin{tabular}{|l|}
14.2 \\
\end{tabular} & & & \\
\hline 6.0 & $B G, M Y, B G, M Y / B O$ & & & \begin{tabular}{|l|}
14.4 \\
\end{tabular} & & & \\
\hline 6.2 & $M Y \angle S I^{2}, M Y, I S U B O$ & & & \begin{tabular}{|l|}
14.6 \\
\end{tabular} & & & \\
\hline 6.4 & $B G^{3} M Y / B O$ & & & 14.8 & & & \\
\hline 6.6 & $M Y^{2} / B O$ & & & 15.0 & & & \\
\hline 6.8 & $M Y / B O$ & & & 15.2 & & & \\
\hline 7.0 & $M Y, B G / B O$ & & & 15.4 & & & \\
\hline 7.2 & $B G / B O$ & & & 15.6 & & & \\
\hline 7.4 & $B G \mid B O$ & & & 15.8 & & & \\
\hline 7.6 & $M Y / B O$ & & & 16.0 & & & \\
\hline 7.8 & $M Y, B G / B O$ & & & 16.2 & & & \\
\hline 8.0 & $P S \mid B O$ & & 1 & \begin{tabular}{|l|}
16.4 \\
\end{tabular} & & & \\
\hline 8.2 & $B G \mid B O$ & & $10^{\circ}$ & 16.6 & & & \\
\hline
\end{tabular}

NOTES: eg epoxy marks, rebar placement, photos...etc 


\section{APPENDIX E - Glacier Bay Intertidal Monitoring Access Database}

\section{ACCESS GENERAL INFORMATION}

\section{Getting Started with Access}

Access is a relational database. This means that you can store information in multiple tables and relate the tables to each other. In other words, if your database had one table with names and addresses and another table with names and phone numbers, you could type in the phone number and Access would give you address of that person (or vice versa). Thus, Access could also tell you at which sites certain species were found or all the sites that contain a certain percentage of a particular substrate.

\section{Database Objects}

There are six types of objects in Access: Tables, Queries, Forms, Reports, Macros and Modules (fig. E1). In the figure below, each of these objects will resemble the tab of a hanging file or a manila folder. Objects consist of the data and the tools you will need in Access. In order to work with one of these objects, click once on the "tab" and a list of all existing files for the database will appear.

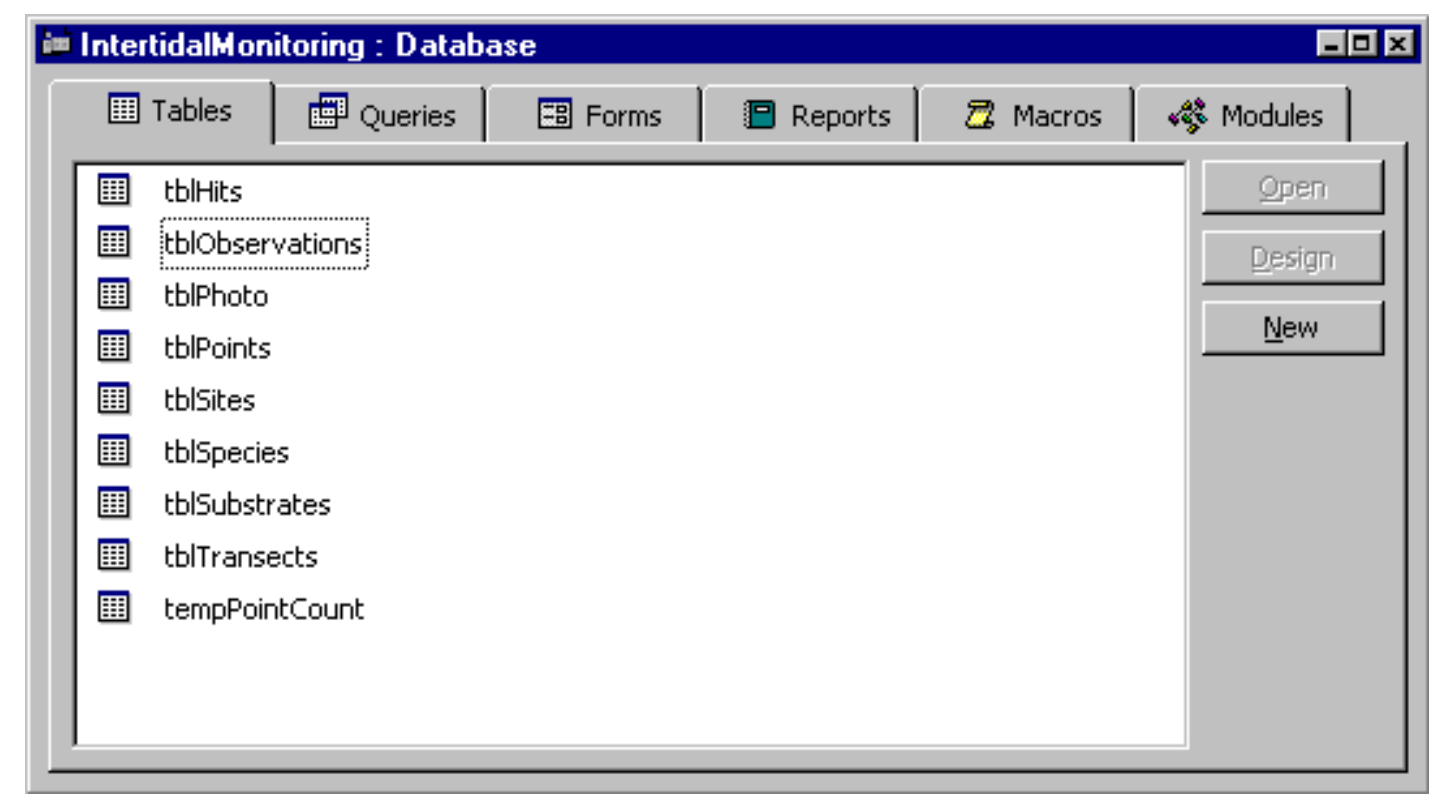

Figure E1. Illustration of the six types of objects found in Access: Tables, Queries, Forms, Reports, Macros and Modules. 


\section{Tables}

Raw data are stored in tables. Any changes made within a table will directly affect the data stored in that table. Because it is possible to permanently change the entire data set when working in table view, it is strongly recommended that you do not work directly in a table unless absolutely necessary. A better alternative is to work with forms and queries.

\section{Forms}

Forms show raw data from tables organized in a more "presentable" or customized format. Generally this means that the data presented in Forms mimics the layout of the datasheets used in the field, facilitating the data entry process.

The data shown in Forms are still raw data and any changes to the data made in Forms will be permanently saved. Although the integrity of the data can still be compromised when using Forms, it is preferable to perform data entry or changes to the data in Forms view. The possibilities of altering large portions of data are greatly reduced.

\section{Queries}

Queries are questions that you ask the database and they are used to extract information (e.g. At what sites can Nucella be found?). Queries are very flexible and by designing exactly what you need you can search, sort and retrieve specific data. Access has many types of queries, but generally the most commonly used queries are: Select, Update, Append and Delete Queries.

\section{Select Queries}

A select query, as you might guess, selects information from one or more tables based on criteria that you specify. Once the query has run, the specific information that you asked for is displayed in a table.

\section{Update, Append, and Delete Queries}

All of these queries are action queries. Action queries allow you to change the data in existing tables and you can change many records with a single operation. Be very careful when using action queries!! The best method when using an action query is to design your query as a select query, run the query and look at the records that you have selected. If your query selected the records that you want to act upon, then change the query to an action query. To change the type of query, click on the Query pull down menu and select the type of query you need (fig. E2). Next you can use the Query Design Pane to specify what you need updated, deleted or appended. 


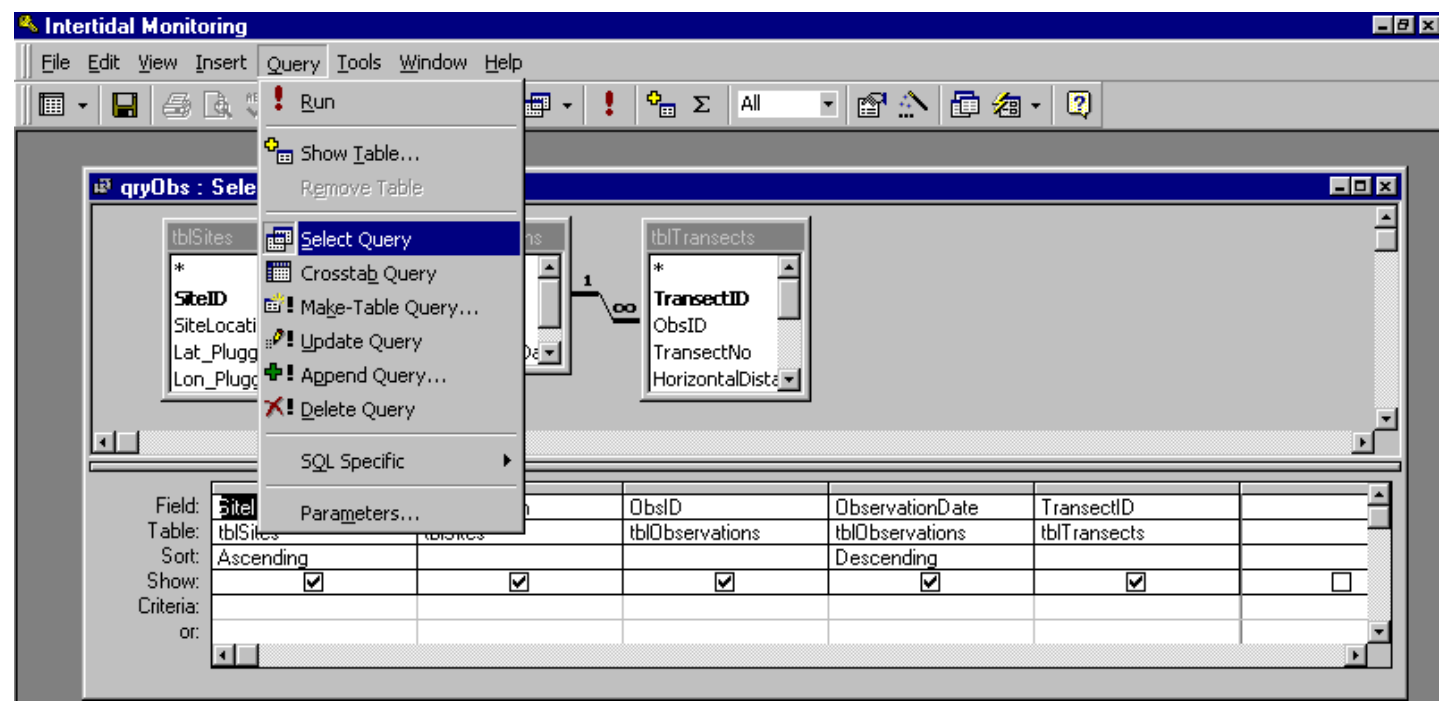

Figure E2. Showing the procedure to change the type of query.

\section{Saving Queries and Naming Queries}

Since queries are frequently used, it is easy to end up with too many of them saved into the database, therefore it is important to only save the queries that will be regularly used. If you create a query that will be used only once do not save it. Attempt to give the queries that you save a very specific or informative name ...so that it is easy to tell what they do when you look at them later (e.g., fig. E3).

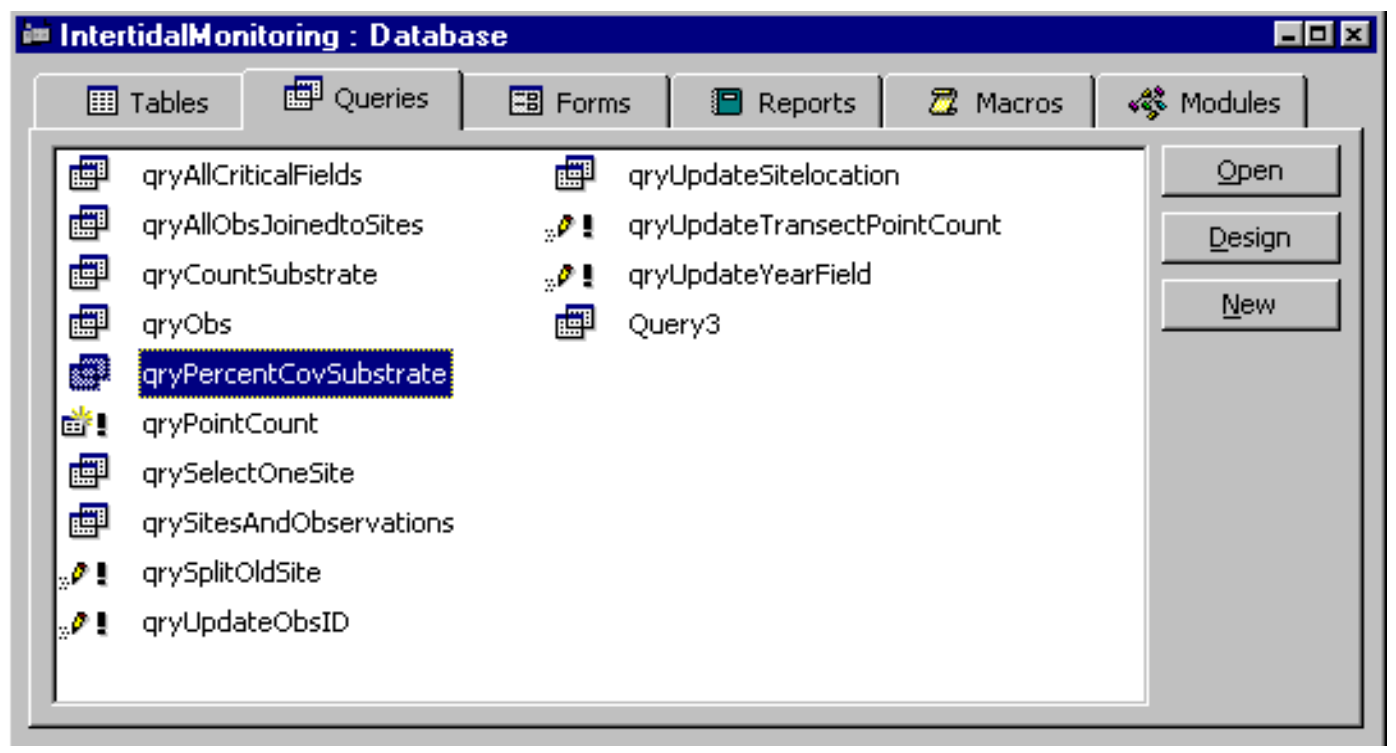

Figure E3. Some of the queries in the intertidal monitoring database. Note that the names begin with "qry." 


\section{DATA ENTRY PROCEDURES}

Upon opening the Intertidal Monitoring database you see the screen displayed in figure E4. Select the "Edit Data" button to begin data entry.

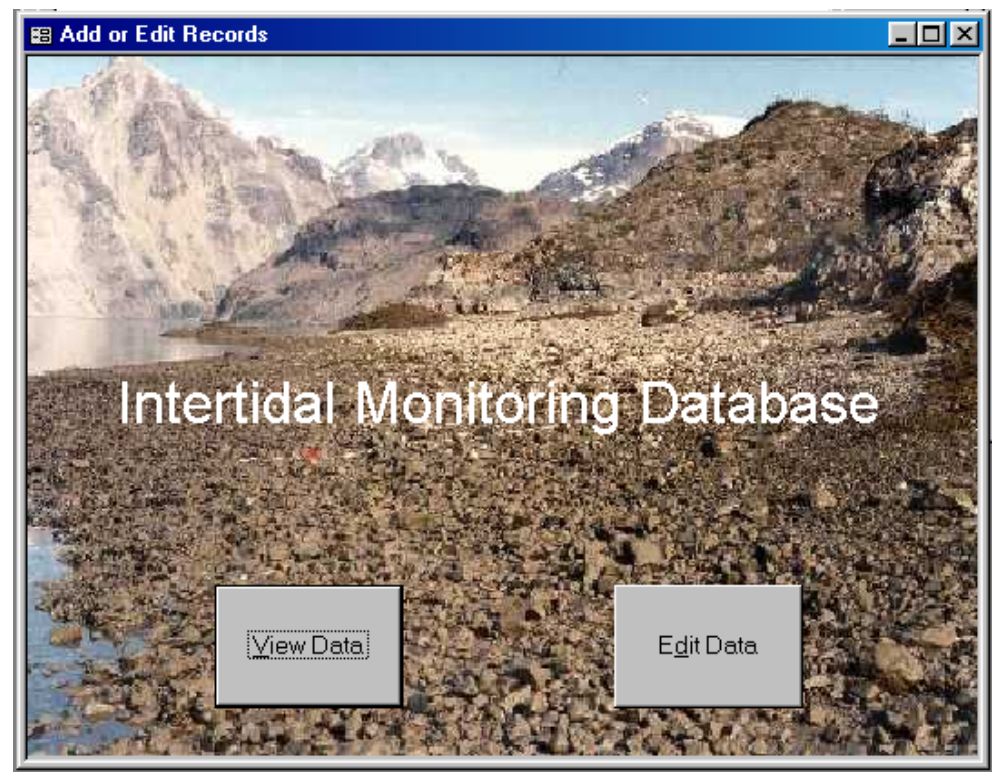

Figure E4. Screen displayed upon opening Intertidal Monitoring database.

The screen should now appear as shown in figure E5. To add observation photos, click on "The Add Observation Photos" button. If you wish to begin data entry for a new site, click the "Enter Data for New Site and Year" button. If you have already begun data entry for a site or if you wish to edit data for a previously entered site, click the "Edit Data for an Existing Site and Year" button.

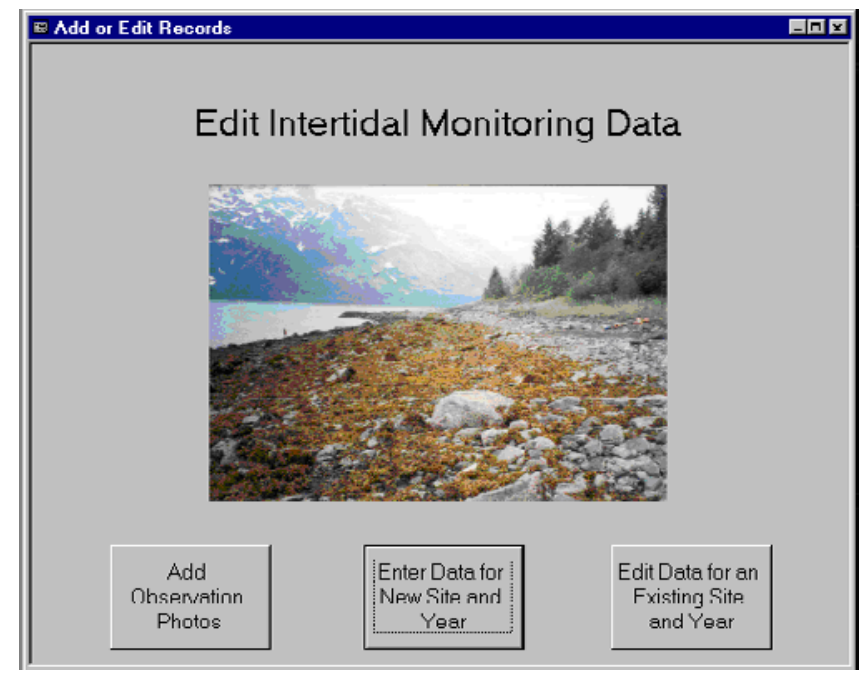

Figure E5. The form that routes you to various ways to edit and enter data. 


\section{a) Edit Data for a New Site and Year}

After you click the button (shown in fig. E5) Access will prompt you for the SiteID. There is a pull-down menu (fig. E6) or you can type in the 3-digit site number. Next Access will ask you for the Observation Date (fig. E6), simply type the date the transects were run.
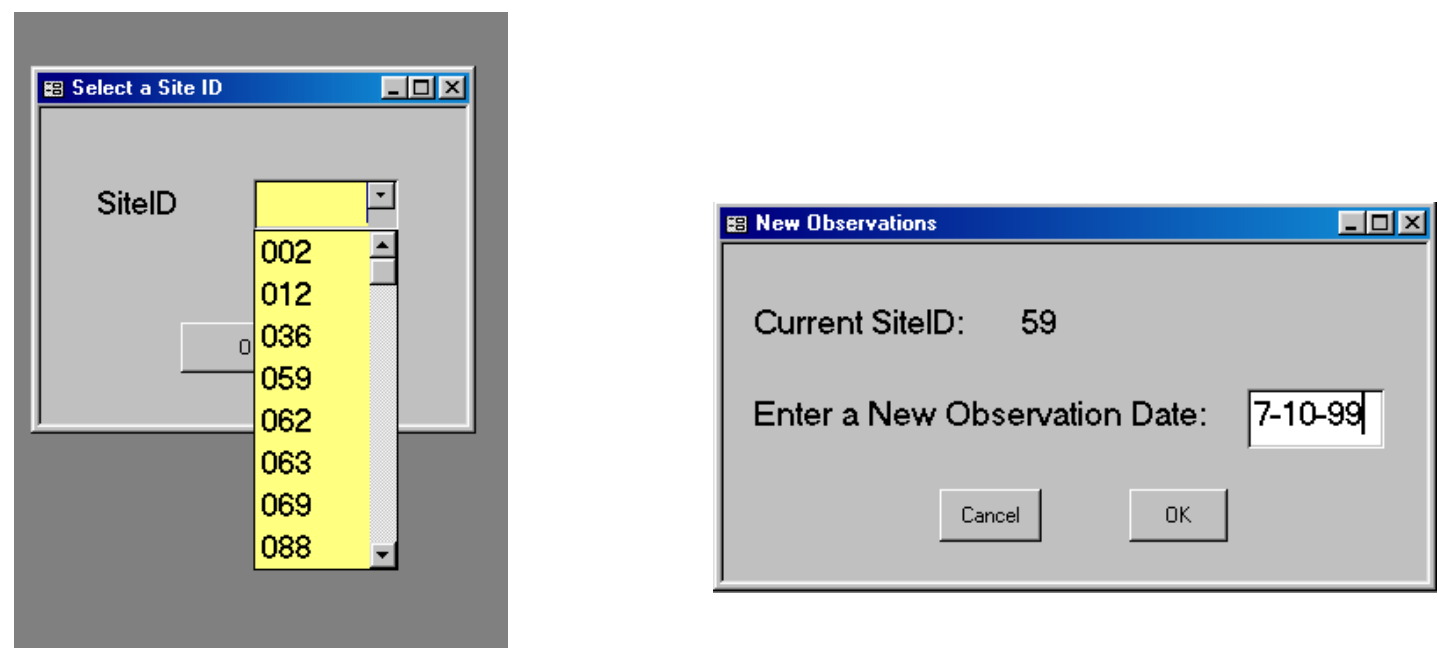

Figure E6. As you enter data for a new site and year, Access will ask for the SitelD and then the Observation Date.

Next the data entry form will appear (fig. E7). The form automatically opens to transect 1 . Enter the transect length, the horizontal distance, and the slope. 


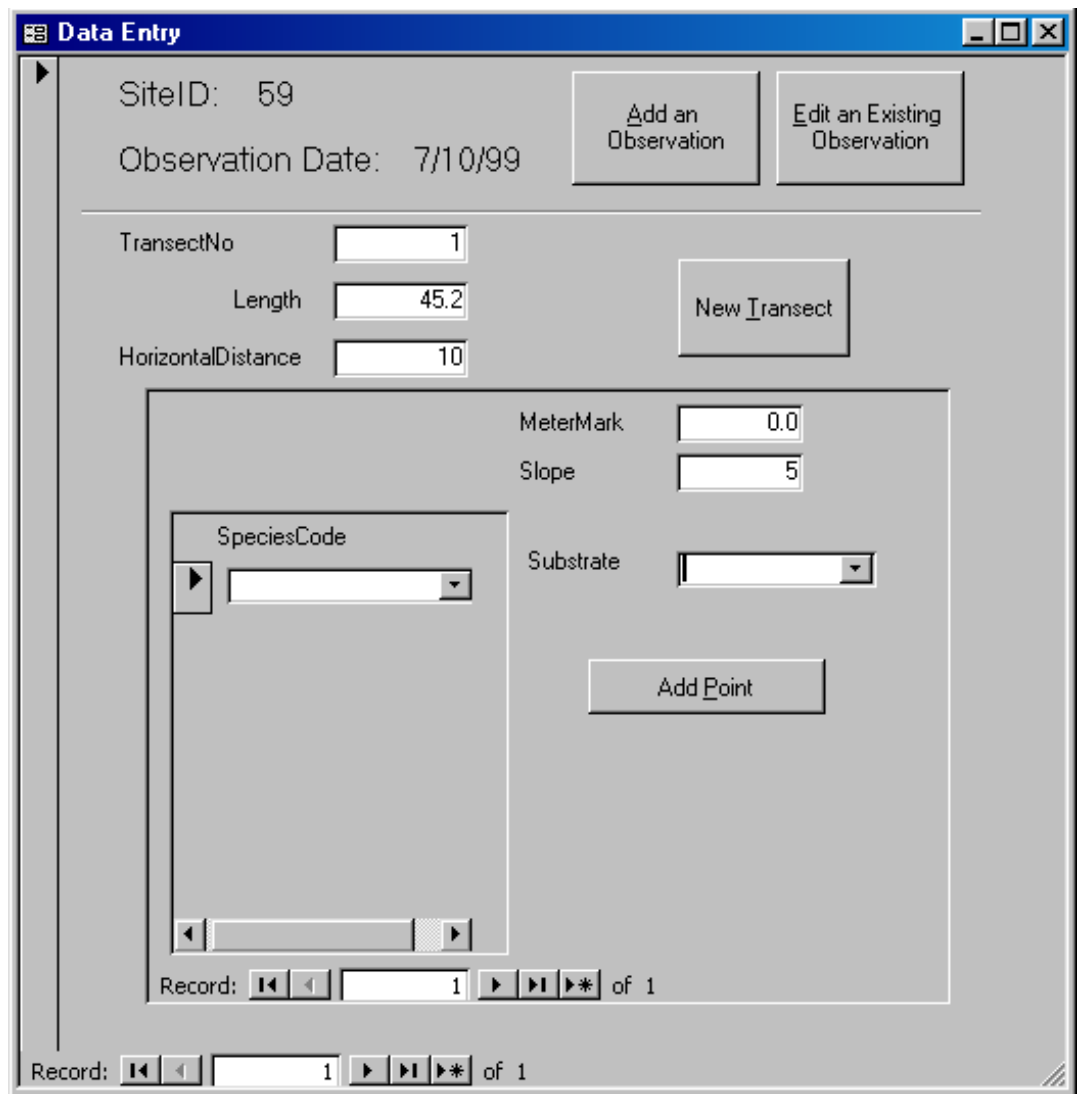

Figure E7. The data entry form.

Then you begin entering data. Each point must have a substrate. Type in any species that were found on that point. You can type in the species code or use the pull-down menu to select a species (Note: Access will not let you type in a species that is not in the pull-down menu. This will hopefully avoid data entry errors from typos). If there were multiple species (or multiple hits of the same species) each hit needs to be entered. For example, figure E8 shows the data entry form for: $\mathrm{FU}^{3}$, LSI, FU, BG / BO on meter mark 2.6.

Once you have entered the species and substrate for a point, click the "Add Point" button. A new point will be added, the meter mark will automatically be $0.2 \mathrm{~cm}$ more and the point will have the same slope and the same substrate as the previous point.

It is often easier to do data entry without using the mouse and these shortcuts may be helpful:

- If the cursor is in Species Code: "Ctrl" and "Tab" (at the same time) will take you to Substrate

- If the cursor is in Substrate: "Enter" once takes you to Add Point, "Enter" again, adds a new point and puts your cursor in Substrate of the new point. 
You can move between points or transects by using the arrows shown in figure E8.

To add another transect at the same site, click the "New Transect" button. Access will automatically add 1 to the transect number. Begin data entry.

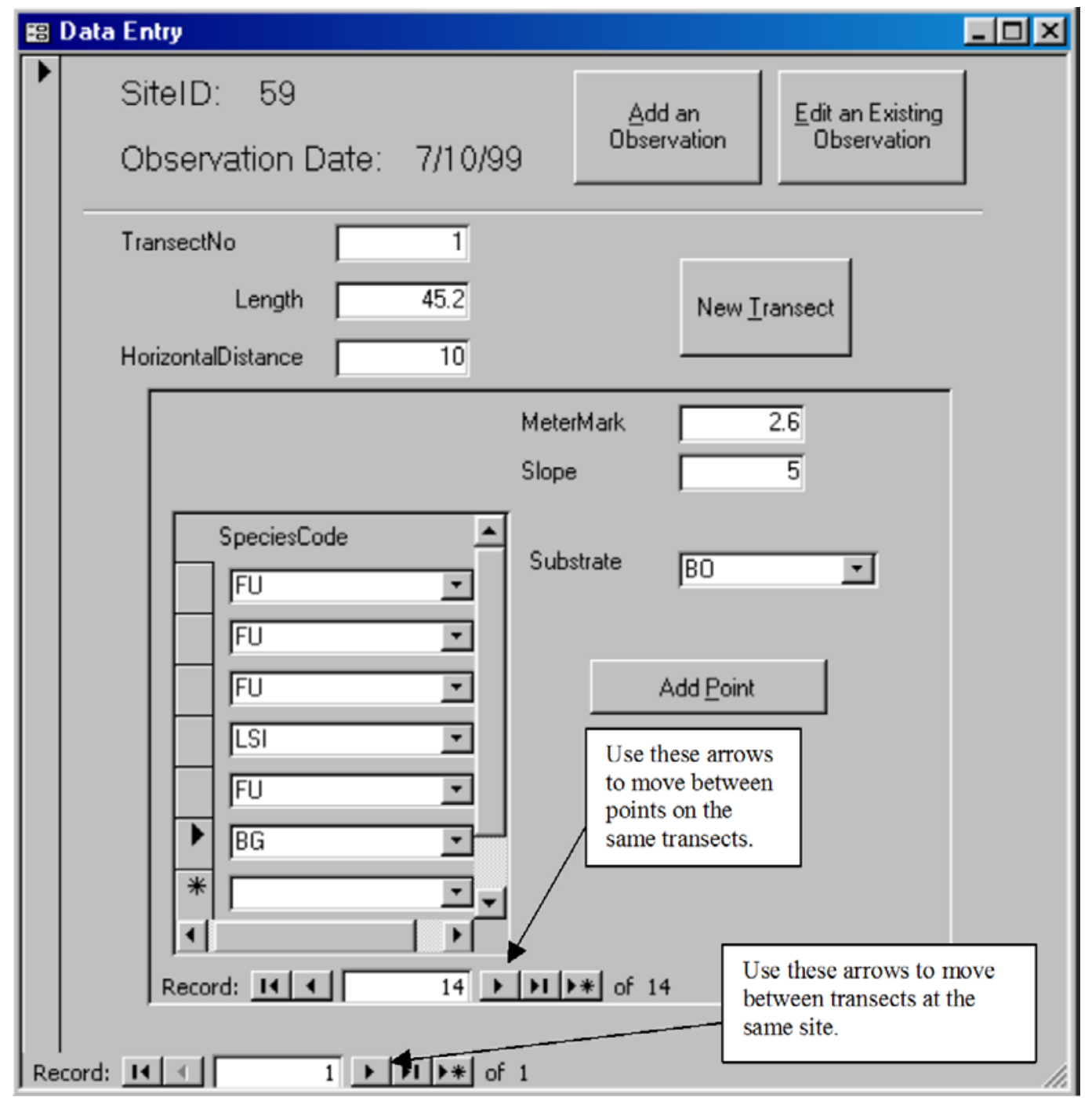

Figure E8. Data entry form after data for meter mark 2.6 (FU3, LSI, FU, BG / BO) have been entered.

\section{b) Edit Data for an Existing Site and Year}

Use this option if you have already entered some data for a site (for example one transect, or part of a transect) and you would like to continue entering data for that site or you would like to edit the data that has already been entered. 
At the Edit Data prompt (fig. E5) click on the button to edit data for an existing site and year. A form will appear (fig. E9) where you can use a pull-down menu to choose the observation data and site number that you wish to edit.

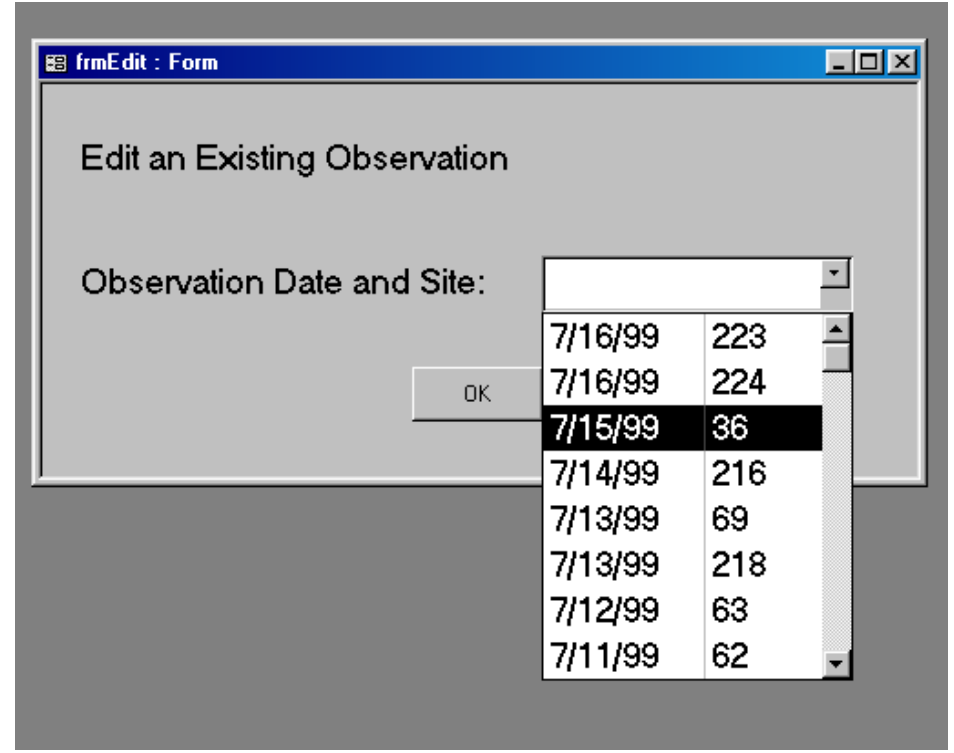

Figure E9. The prompt to edit data for a site and observation date that already exist in the database.

When you click "OK" the data entry form will appear (fig. E8) and you can make the changes that need to be made. Remember you can move between transects and points using the arrows shown in figure E8. 


\section{EXPORTING DATA}

NOTE: It is very easy to export any table from Access, however, since Access is a relational database all the information you may want to export may be spread out in several tables. In order to export information from multiple tables, you need to write or use a select query. The select query will create a custom table with all the information that you need so that it can be exported.

To Export a table:

Go to the Intertidal Database window (fig. E1). If you cannot see this window, use the Window pull-down menu at the top of the screen and choose "Intertidal Monitoring: Database." In the Intertidal Database window, click on the Tables tab. List of all the tables in the database will appear. To export a table, click on it to highlight it. Next, use the File pull down-menu at the top of the screen and choose Save As/Export.

\section{To Export from a Query:}

Go to the Intertidal Database window (fig. E1). If you cannot see this window, use the Window pull-down menu at the top of the screen and choose "Intertidal Monitoring: Database." In the Intertidal Database window, click on the Queries tab. If the query you want already exists, simply double click on the query, wait for Access to run the query and create a table. Then from the File pull-down menu choose Save As/Export to export the table. You could also write a new query and export the table that you create.

For example to export all of the data for the entire database: Go to the Intertidal Database window (fig. E1). If you cannot see this window, use the Window pull-down menu at the top of the screen and choose "Intertidal Monitoring: Database." In the Intertidal Database window, click on the Queries tab. Double click on the "qryAllCriticalFields" query. It will take Access a moment to run. A table will show all the data that will be created. Use the File pull down menu and chose Save As/Export. 
This page left intentionally blank 
Publishing support provided by the U.S. Geological Survey Publishing Network, Tacoma Publishing Service Center

For more information concerning the research in this report, contact the Director, Alaska Science Center U.S. Geological Survey 4210 University Dr.

Anchorage, Alaska 99508-4650

http://alaska.usgs.gov 


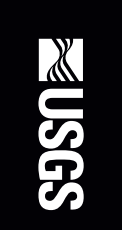

$\overline{\bar{\Xi}}$

$\stackrel{\square}{<}$

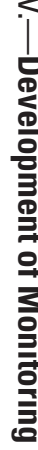

웅

뭉

高

올

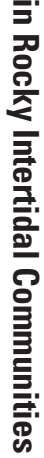

음

몽

ब.

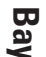

을

낓

흘

궁

通

윢

䒰 